


\title{
THE QUINTIC GAUSS SUMS
}

\author{
Dissertation \\ zur Erlangung des Doktorgrades \\ der \\ Mathematisch-Naturwissenschaflichen \\ Fakultäten \\ der Georg-August Universität zu \\ Goettingen
}

\author{
vorgelegt von \\ Léopold Fossi Talom \\ Aus Bandjoun/ Yom V, Kamerun
}

Göttingen 2002 
D7

Referent: Prof. S.J. Patterson

Korreferentin: Frau Prof. Dr. I. Kersten.

Tag der Disputation: 


\section{Contents}

$\begin{array}{ll}\text { List of Figures } & 7\end{array}$

Chapter 1. INTRODUCTION 9

Chapter 2. Generalities on Cyclotomic Fields 17

$\begin{array}{ll}\text { 1. Introduction } & 17\end{array}$

2. Power residue Symbol 24

3. The primary choice of the prime factor in $\mathbb{Q}\left(\zeta_{5}\right) \quad 26$

$\begin{array}{ll}\text { Chapter 3. GAUSS SUMS } & 29\end{array}$

1. Gauss sums in number fields 29

2. Factorization of Gauss sums 36

3. Gauss sums over finite fields and the Davenport-Hasse relations $\quad 39$

4. The Gauss sum and Jacobi sum as complex numbers 40

5. The moment Gauss sums and the uniform distribution 45

Chapter 4. CYCLOTOMIC CRYSTAL 49

1. Introduction 49

2. The cyclotomic case 51

3. The vertices 56

4. The geometry and the combinatoric $\quad 59$

5. Generalization 65

6. Cyclotomy revisited 69

Chapter 5. THE GAUSS SUMS AND THE FUNDAMENTAL
DOMAIN

1. First identity $\quad 77$

$\begin{array}{lr}\text { 2. Second identity } & 79\end{array}$

3. Third identity 80

4. Cassels' formula $\quad 82$

5. A generalized of a formula of Cassels' type 83

6. Conjectures on the partial sums of the Gauss sums 89

$\begin{array}{lll}\text { Chapter 6. APPENDIX } & 105\end{array}$

1. The Gauss periods or the Lagrange resolvents 105

2. The quadratic Gauss sums 109

3. Algorithm for the computation of the Gauss sums 111

$\begin{array}{ll}\text { 4. data } & 115\end{array}$

$\begin{array}{ll}\text { Bibliography } & 143\end{array}$ 



\section{List of Figures}

Graphic of the arguments of Gauss sums for $\pi \equiv 1$

$\left(\bmod \lambda^{3}\right) \quad N(\pi) \leq 20000$

Graphic of the arguments of Gauss sums for $\pi \equiv 1$

$\left(\bmod \lambda^{3}\right) \quad N(\pi) \leq 40000$

frequency in each 1 degree on the circle

$g(X)=\frac{1}{X^{6 / 5}} \sum_{\substack{n \leq X \\ 0<X<40.000}} \Omega\left(S_{0}, \varepsilon, \pi\right) \log (N(\pi))$

$g(X)=\frac{1}{X} \sum_{\substack{n \leq X \\ 0<X<40.000}} \Omega\left(S_{0}, \varepsilon, \pi\right) \log (N(\pi))$

$g(X)=\frac{1}{X} \sum_{\substack{n \leq X \\ 0<X<40.000}} \Omega\left(S_{0}, \varepsilon, \pi\right) \log (N(\pi))\left(1-\frac{N(\pi)}{X}\right)$

$g(X)=\frac{1}{X} \sum_{\substack{n \leq X \\ 0<X<40.000}} \Omega\left(S_{0}, \varepsilon, \pi\right) \log (N(\pi)) \quad$ and $\quad f(X)=$

$\frac{1}{X} \sum_{\substack{n \leq X \\ 0<X<40.000}} \Omega\left(S_{0}, \varepsilon, \pi\right) \log (N(\pi))\left(1-\frac{N(\pi)}{X}\right)$

$$
g(X)=\frac{1}{X} \sum_{\substack{n \leq X \\ 0<X<40.000}} \Omega\left(S_{0}, \varepsilon, \pi\right) \log (N(\pi))\left(1-\frac{N(\pi)}{X}\right)
$$

$$
\text { and } \sum_{\substack{n \leq X \\ 0<X<40.000}} \Omega\left(S_{0}, \varepsilon, \pi\right) \log (N(\pi))\left(1-\frac{N(\pi)}{X}\right)
$$

$f(X)=X^{-1 / 2} \sum_{\substack{n \leq X, 0<X<50.000}} \mu(n) n^{1 / 2}$

$$
f(X)=\frac{1}{X} \sum_{\substack{n \leq X, 0<X<50.000}} \mu(n) n^{1 / 2}
$$

$$
\sum_{\substack{\pi \equiv 1(\bmod \lambda 3), N(\pi) \leq X, X \leq 40000}} \mathfrak{g}(1, \varepsilon, \pi) \log (N(\pi))
$$

$$
\text { and } \sum_{\substack{\pi \equiv 1\left(\bmod \lambda^{3}\right), N(\pi) \leq X, X \leq 40000}} \mathfrak{g}(1, \varepsilon, \pi) \log (N(\pi))\left(1-\frac{N(\pi)}{X}\right)
$$

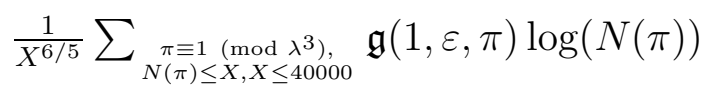

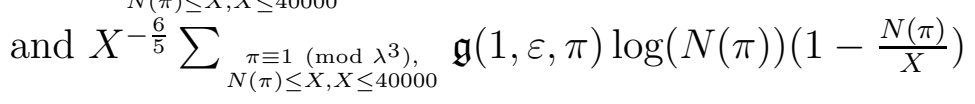

$$
f(X)=X^{-\frac{6}{5}} \sum_{\substack{\pi \equiv 1 \\ N(\pi) \leq X, X \leq 40000 \\ 7}} \frac{\mathfrak{g}(1, \varepsilon, \pi) \log (N(\pi))}{\Omega\left(S_{0}, \varepsilon, \pi\right)}
$$




$$
\text { and } \quad X^{-\frac{6}{5}} \sum_{\substack{\pi \equiv 1\left(\bmod \lambda^{3}\right), N(\pi) \leq X, X \leq 40000}} \frac{\mathfrak{g}(1, \varepsilon, \pi) \log (N(\pi))}{\Omega\left(S_{0}, \varepsilon, \pi\right)}\left(1-\frac{N(\pi)}{X}\right)
$$

$18 \quad \sum_{\substack{\pi \equiv 1\left(\bmod \lambda^{3}\right), N(\pi) \leq X \times X \leq 40000}} \frac{\mathfrak{g}(1, \varepsilon, \pi) \log (N(\pi))}{\Omega\left(S_{0}, \varepsilon, \pi\right) N(\pi)}$

$$
X^{-\frac{6}{5}} \sum_{\substack{\pi \equiv 1 \\ N(\pi) \leq X, X \leq 40000}} \frac{\mathfrak{g}(1, \varepsilon, \pi) \log (N(\pi))}{N(\pi)} \text { and }
$$




\section{CHAPTER 1}

\section{INTRODUCTION}

In 1801, Gauss in his Disquisitiones Arithmeticae introduced the following sum

$$
G_{2}(m ; k)=\sum_{n=0}^{k-1} e^{2 \pi i m n^{2} / k} .
$$

where $m$ and $k$ are coprime.

This sum has a remarkably simple dependence on its arguments. In the case $k$ odd, it has values as $\pm \sqrt{k}$, or $\pm i \sqrt{k}$. Gauss discovered in 1801 that in fact for $\mathrm{k}$ odd:

$$
G_{2}(1 ; k)=\left\{\begin{array}{cc}
\sqrt{k} & \text { if } \quad k \equiv 1 \bmod 4 \\
i \sqrt{k} & \text { if } \quad k \equiv-1 \bmod 4
\end{array}\right.
$$

He only found a proof 4 years later, in August 1805. He also determined these values for $k \equiv 0,2 \bmod 4$. There are nowadays many different proofs of this theorem. See for example $[\mathbf{S . I}],[\mathbf{K} . \mathbf{L}],[\mathbf{E} . \mathbf{T}]$

One important property is that Gauss sums generate quadratic subfields of cyclotomic fields. Indeed one can verify easily that for integer $k$ odd,

$$
G_{2}(m, k)^{2}=(-1)^{\frac{k-1}{2}} k=k^{*} \text {. }
$$

Therefore the quadratic field $\mathbb{Q}\left(\sqrt{\left((-1)^{\frac{k-1}{2}} k\right)}\right)$ is contained in $\mathbb{Q}\left(e^{\frac{2 \pi i}{k}}\right)$. This fact is one of the properties discovered by Gauss before he determined the correct sign for the Gauss sums. He used it to prove the law of quadratic reciprocity. He considers for two distinct primes $p, q$, and if (:) is the Legendre symbol, then the expression

$$
G_{2}(1, p)^{q-1} \equiv G(q, p)^{2 \frac{q-1}{2}}=p^{* \frac{q-1}{2}} \equiv\left(\frac{p^{*}}{q}\right) \quad \bmod q
$$

thus

$$
\begin{aligned}
G_{2}(1, p)^{q} & \equiv G(q, p) \quad(\bmod p) \\
& \equiv\left(\frac{q}{p}\right) G(1, p) \quad(\bmod p) \\
& \equiv\left(\frac{p^{*}}{q}\right) G(1, p) \quad(\bmod p)
\end{aligned}
$$

and so multiply both side of the last equation above by $\left(\frac{q}{p}\right)$ we obtain the desired result:

$$
\left(\frac{q}{p}\right)\left(\frac{p}{q}\right)=(-1)^{\left(\frac{q-1}{2}\right)\left(\frac{p-1}{2}\right)}
$$

Another proof is obtained as follows. The Chinese Remainder Theorem give the values of Gauss sums for composite odd numbers $p q$ and the definition of $G_{2}(1, p)$ 
for different values of prime $p$ is known for congruence classes modulo 4 of the odd numbers $p, q$, and $p q$ so that in plugging in all that within the following identity:

$$
G_{2}(1, p q)=\left(\frac{p}{q}\right)\left(\frac{q}{p}\right) G_{2}(1, p) G_{2}(1, q) ;
$$

we deduce the law of quadratic reciprocity as:

$$
\left(\frac{p}{q}\right)\left(\frac{q}{p}\right)=(-1)^{\frac{p-1}{2} \frac{q-1}{2}} .
$$

This is another proof of the law of quadratic reciprocity.

A more general Gauss sum is the following.

$$
G_{n}(m ; k)=\sum_{\ell=0}^{k-1} e^{\left(2 \pi i m \ell^{n} / k\right)} .
$$

We see that for $k$ prime and $\operatorname{gcd}(k, m)=1$

$$
G_{n}(m ; k)=\sum_{\chi: \chi \neq 1} \tau_{n}(\chi)
$$

where the $\chi$ are all non trivial character modulo $k$ of order $\ell$ and

$$
\tau_{n}(\chi)=\sum_{a \bmod k} \chi(a) \exp (2 \pi i a n / k)
$$

The cubic Gauss sum, also called Kummer sum after E.E. Kummer, is

$$
G_{3}(1, p)=\sum_{n=1}^{p-1} \exp \left(\frac{2 \pi i n^{3}}{p}\right)
$$

can be expressed as $G_{3}=\tau_{3}(\chi)+\tau_{3}(\bar{\chi})$ where $\chi$ is a cubic character on $\mathbb{F}_{p}$, and

$$
\tau_{3}(\chi)=\sum_{n=1}^{p-1} \chi(n) \exp \left(\frac{2 \pi i n}{p}\right)
$$

The sum $G_{3}(1, p)$ is indeed a root of the cubic period polynomial $f_{p}(X)=X^{3}-$ $3 p X-p L$ where $4 p=L^{2}+27 M^{2}$. This polynomial has exactly one root in each of the interval $[-2 \sqrt{p},-\sqrt{p}],[-\sqrt{p}, \sqrt{p}]$ and $[\sqrt{p}, 2 \sqrt{p}]$, and the question here is to know which of them contains the root $G_{3}$. The quotient

$$
\frac{\tau_{3}(\chi)}{\left|\tau_{3}(\chi)\right|}
$$

lies on the unit circle. Looking at their arguments $\theta_{p}$, Kummer observed here that the investigation on the 45 cases of prime $p$ less than 500 gives the frequency $3: 2: 1$ respectively and he suggested that

$$
\sum_{\substack{p \equiv 1 \bmod 3, p<x}} \chi_{h}\left(\left|\theta_{p}\right|\right)=\frac{W_{h} x}{2 \log x}+o\left(\frac{x}{\log x}\right) \quad h=1,2,3,
$$

where $\chi_{1}$ (resp. $\left.\chi_{2}, \chi_{3}\right)$ is the characteristic function of the interval $\left(0, \frac{\pi}{3}\right]$ (resp. $\left(\frac{\pi}{3}, \frac{2 \pi}{3}\right],\left(\frac{\pi}{3}, \pi\right]$ and $W_{h}=\frac{1}{2}, \frac{1}{3}, \frac{1}{6}$ for $h=1,2,3$ respectively. The study of the frequence 
analysis of this arguments was done by Lehmer [EL], Goldstine and Von Neumann [G.VN]. They found, for $p<1000$ a different distribution approximately $4: 3: 2$. This led one to doubt about the conjecture of Kummer. The same investigation led one observed that the arguments $\theta_{p}$ are likely equidistributed in the interval $(0, \pi)$ for the Lebesgue measure. Then following a suggestion of Davenport [Da], Moreno [MO] in 1974 proved that if $I \in(0, \pi]$ is a subinterval, then

$$
\sum_{p \equiv 1 \bmod 3, p<x} \chi_{I}\left(3 \theta_{p}\right)=\frac{|I| x}{2 \log x}+o\left(\frac{x}{\log x}\right)
$$

where $|I|$ is the Lebesgue measure of $I$. This just proves that the arguments of the $\tau_{3}(\chi)^{3}$ are uniformly distributed in the three intervals listed above. The idea of Moreno is that, the character $\chi=\left(\frac{\pi}{)}\right)$ is associated to a factor $\pi \mid p$, such that $\pi \equiv-1 \bmod 3$ in the field of cubic roots of unity $\mathbb{Q}\left(\zeta_{3}\right)$. He then observed that $\exp (i \theta(\pi))=p^{\frac{-3}{2}} \tau_{3}^{3}(\chi)$ is a Grössencharcter in $\mathbb{Q}\left(\zeta_{3}\right)$, then from Hecke's theory of L-functions, it follows

$$
\sum_{N \omega \leq X} \exp (\operatorname{in} \theta(\omega))=o\left(\frac{X}{\log X}\right)
$$

as $X \rightarrow \infty$, for each integer $n \neq 0$, and this is equivalent to the uniform distribution of $\tau_{3}^{3}(\chi)$. This proof has led one to seriously looking for a proof to disprove the Kummer's conjecture. Kubota [KB1] [KB2] has indicated a method for obtaining some asymptotic results on the determination of the Gauss sums. This was based mostly on his theory of automorphic functions. Indeed if we define

$$
\Gamma=\left\{\gamma=\left(\begin{array}{ll}
a & b \\
c & d
\end{array}\right) \in S L\left(\mathbb{Z}\left[\zeta_{3}\right]\right) ; \quad \gamma \equiv I \bmod 3\right\}
$$

then

$$
\psi(\gamma)=\left\{\begin{array}{lll}
\left(\frac{c}{a}\right)_{3} & \text { if } \quad c \neq 0 \\
1 & \text { if } \quad c=0
\end{array}\right.
$$

We see that $\psi$ is a character on $\Gamma$ and $\Gamma$ acts discontinuously on the upper half space $H=\mathbb{C} \times \mathbb{R}_{+}^{\times}$as follows. A point $u=(z, v)$ in $H$ is represented by the matrix

$$
\left(\begin{array}{cc}
z & -v \\
v & \bar{z}
\end{array}\right)
$$

and for $w \in \mathbb{C}$, we write

$$
\tilde{w}=\left(\begin{array}{cc}
w & 0 \\
0 & \bar{w}
\end{array}\right)
$$

then the action of $\Gamma$ on $H$ is given by:

$$
\gamma(u)=(\tilde{a} u+\tilde{b})(\tilde{c} u+\tilde{d})^{-1}, \quad \gamma=\left(\begin{array}{ll}
a & b \\
c & c
\end{array}\right) .
$$

Following Selberg, the Eisenstein series corresponding to the cusp $\infty$

$$
E(u, s)=\sum_{\gamma \in \Gamma_{\infty} \backslash \Gamma} \overline{\psi(\gamma)} v(\gamma(w))^{s}
$$

for $\operatorname{Re}(s)>2$ and $u=(z, v) \in H$, and where

$$
\Gamma_{\infty}=\{\gamma \in \Gamma ; \gamma(\infty)=\infty\}
$$


$E(u, s)$ has a meromorphic continuation to the whole s-plane. If we complement $E(u, s)$ by the further three Eisenstein series corresponding to the remaining inequivalent essential cusp, then there is a functional equation connecting the values at $s$ and at $2-s$. On forming the Fourier expansion of $E(u, s)$ with respect to $\Gamma_{\infty}$, we find that the coefficients are Dirichlet series satisfying similar functional equations and whose coefficients are cubic Gauss sums. The theory shows that these Dirichlet series are regular in the half-plane $\operatorname{Re}(s)>4 / 3$. In particular, the regularity at $s=3 / 2$ together with a well-known tauberian theorem gives

$$
\sum_{N(\alpha) \leq X} \frac{g_{3}(\alpha)}{\left|g_{3}(\alpha)\right|}=o(X) \quad \text { as } \quad X \rightarrow \infty
$$

where $\alpha \equiv 1 \bmod 3$ in $\mathbb{Z}\left[\zeta_{3}\right]$ and

$$
g_{3}(\alpha)=\sum_{x \bmod \alpha}\left(\frac{x}{\alpha}\right)_{3} \exp \left(\pi i \operatorname{Tr}_{K / \mathbb{Q}}\left(\frac{x}{\alpha}\right)\right)
$$

where $\operatorname{Tr}_{K / \mathbb{Q}}$ is the trace of the linear map within the $\mathbb{Q}$-vector space

$$
K=\mathbb{Q}(\zeta) \rightarrow K ; \quad y \mapsto y x \text { for } 0 \neq x, y \in K .
$$

The norm $\mathrm{N}_{K / \mathbb{Q}}$ is also defined to be the determinant of the same map. This definition is modified in case $K / \mathbb{Q}$ is a Galois extension.

Patterson [SJP3], [SJP4] between 1977 and 1978 developed the work of Kubota and established the cubic analogue of the theta series. This enabled Patterson [SJP1] in 1978, and later in 1979 Heath-Brown and Patterson( for general result) $[\mathbf{H P}]$ to prove that the arguments of Gauss sums are uniformly distributed according to the Weyl criterion for uniform distribution on the unit circle. Patterson essentially proved that:

$$
\sum_{\substack{N(\pi) \leq X \\ \pi \equiv a \bmod \alpha}}\left(\frac{g_{n}(\pi)}{\left|g_{n}(\pi)\right|}\right)^{m}=o\left(\frac{X}{\log (X)}\right) .
$$

for any prime $\pi$, and for any integer $m \neq 0$, where

$$
g_{n}(\pi)=\sum_{x \bmod \pi}\left(\frac{x}{\pi}\right)_{n} e\left(2 \pi i \operatorname{Tr}_{K / \mathbb{Q}}\left(\frac{x}{\pi}\right)\right) .
$$

The symbol

$$
(\dot{-})_{n}
$$

is the $n^{\text {th }}$ power residue symbol.

Indeed if we put

$$
\tilde{g_{n}}(\alpha)=\frac{g_{n}(\alpha)}{\left|g_{n}(\alpha)\right|} \quad\left(=0 \quad \text { if } \quad g_{n}(\alpha)=0\right)
$$

for any ideal $\alpha$.

In 1979 Patterson did prove that if one selects $\alpha$ modulo the $n^{\text {th }}$ powers of units, then

$$
\sum_{N(\alpha) \leq X} \tilde{g_{n}}(\alpha) \omega(\alpha) \Lambda(\alpha)=o(X)
$$

Where $\Lambda$ is the von Mangoldt function in the field $\mathrm{K}$ and here $\omega(c)$ is a Grössencharacter of $\mathrm{K}$ i.e a function on the idèle group which is trivial on the field $\mathrm{K}$. This result is 
a generalization of the previous one by Heath-Brown and Patterson $[\mathbf{H P}]$ in 1979 and is up to now known as the latest most outstanding achievement in the theory of Gauss sums. This proof is ingenious and difficult.

Patterson [SJP3][SJP5], showed that, if we take the Kummer sums $g_{3}(\alpha)$ for any integer $\alpha \equiv 1 \bmod 3$ in $\mathbb{Z}\left[\zeta_{3}\right]$, then a formal application of the Hardy Littlewood methods suggested the asymptotic formula

$$
\sum_{N(\alpha) \leq X} \Re\left(\frac{g_{3}(\alpha)}{\left|g_{3}(\alpha)\right|}\right) \sim \frac{(2 \pi)^{2 / 3}}{5 \Gamma(2 / 3)} \frac{X^{5 / 6}}{\log X} \quad \text { as } \quad X \rightarrow \infty=O\left(X^{\frac{5}{6}+\epsilon}\right) \quad \text { for all } \quad \epsilon>0 .
$$

In general, if $\chi$ is a normalized Hecke character, then for ideal $I$ and prime $\pi$ we have

and

$$
\sum_{N(I) \leq X} \chi(I)=\alpha(\chi) X+o(X)
$$

$$
\sum_{N(\pi) \leq X} \chi(I)=\beta(\chi)\left(\frac{X}{\log X}\right)+o\left(\frac{X}{\log X}\right) .
$$

Using Kummer,s theory of ideal numbers, Stickelberger [S.L] in 1890, proved that the ideal generated by the Gauss sums can be factorized as follows: if $\alpha \in \mathbb{Z}\left[\zeta_{m}\right]$ the

$$
\left(g_{m}(\alpha)\right)=(\alpha)^{\theta}
$$

where theta is the element of the group ring $\mathbb{Q}[\operatorname{Gal}(K / k)]$ defined by

$$
\theta=\frac{1}{m} \sum_{\substack{\operatorname{gcd}(t, m)=1 \\ t \bmod m}} t \cdot \sigma_{-t} \quad \text { and the Galois element is such that } \quad \sigma_{t}(\zeta)=\zeta^{t}
$$

Gauss proof of the quadratic reciprocity law by counting lattice points reappears in the determination of the sign the quadratic Gauss sums. Later, J.W.S. Cassels, in 1969,1970 [J.W.S] and [J.W.S1], proposed a formula for cubic Gauss sums in terms of values of elliptic functions. A student of him, A. D. McGettrick [Mc.G1](1972), reformulated this conjecture in terms of the number of lattice points in certain domain. This conjecture and his biquadratic analogue where proved by C. R. Matthews [Mat].

It is a goal of this thesis to investigate the possibility of the analogue of this formula for the quintic Gauss sums.

We define certain roots of unity $\Omega\left(S_{0}, \varepsilon, \pi\right)$ which generalizes the construction of Cassels. It has certain formal properties analogous to those of Gauss sums $\mathfrak{g}(1, \varepsilon, \pi)$. We investigate whether the quotient of this is a simple function in the sens that it can be obtain by information of a finite number of places. the evidence shows that it is probably not the case and the statistical investigation shows evidence of unexpected, but very interesting structure. We give two conjecture on the quintic Gauss sums, namely, on the asymptotic behavior of it partial sums. 
In Chapter I, we give a brief description of the cyclotomic fields. We outline the key properties necessary for the introduction of the Gauss sums. We expose the decomposition of prime in general cyclotomic field and define the norm residue symbol. We give a definition of a primary element in the fifth cyclotomic field. We use this kind of prime algebraic integers for the computation.

Chapter II describes the basic properties of the Gauss sums. We outline some key properties of the Gauss sums. We state the Davenport-Hasse Theorems, and introduce the Weil Grössencharacter. We study the asymptotic behavior of powers of Gauss sums (of moment of a Gauss sums) and explain a property on the distribution of Gauss sums. The Gauss sums, after being defined purely algebraically, will be regarded as complex numbers via embeddings into $\mathbb{C}$. This has helped to make computational test through a $\mathrm{C}$ program.

In Chapter 3, we describe the fundamental domain. These fields studied here are the prime cyclotomic field. We determine in detail the skeleton of the fundamental domain for the prime cyclotomic field. We find results to characterize the geometry, we prove that there are $\frac{n-1}{2}$ different shapes of faces. This result generalizes the result obtain by Dirichlet for the third cyclotomic field. There are different geometries for the faces of a the fundamental domain. For $n=3$ and 5 we give a picture for the faces of their fundamental domain. For this, we handle it in a very simple way, just by studying subsets instead of using brute force. This has the advantage of generalizing as far as possible. We give two different methods to construct an $n^{\text {th }}$ set for prime $n$. One of these methods is very elementary, based only on the properties of subsets of the $n$ first positive integers. The combinatorics of these geometries are also studied. We discover that they are related to some numbers $\gamma(n, i)$.

In Chapter 4 we describe some identities that could help us to understand the behavior of the Gauss sums. We fix an $n$-set, $S_{0}$, once and for all. We give identities that reflect the properties of Gauss sums. We specialize on the fifth case to do some computation. The results of the computations would be consistent with a generalization of the Cassels-McGettrick formula. This unfortunately does yield at the present stage a conjecture on the determination of the correct sign for the Gauss sums. However, we can explain more on the distribution of certain functions involving the values of Gauss sums. We attempt to verify a conjecture of Patterson. We study the functions

and

$$
H(X)=\sum_{\substack{N(\pi)<X \\ \pi \equiv 1 \bmod \lambda^{3}}} \mathfrak{g}(1, \varepsilon, \pi) \log (N(\pi))
$$

$$
\tilde{H}(X)=\sum_{\substack{N(\pi)<X \\ \pi \equiv 1 \bmod \lambda^{3}}} \frac{\mathfrak{g}(1, \varepsilon, \pi) \log (N(\pi))}{\Omega(\varepsilon, \pi)}
$$

Both functions have more or less the same shape and contrary to our expectation, that it would behave like the summation function of the Möbius function, but in fact its behavior is quite different. The observations are graphically consistent with the conjecture that

$$
H(X)>0 \text { as well as } \tilde{H}(X)>0
$$


for $X$ large. This is would probably behave like other functions, known in the theory of elementary number theory, when we replace $\mathfrak{g}(1, \varepsilon, \pi)$ by the Möbiüs function . We also confirm a suggestion of Patterson, that

$$
H(X) \sim c . X^{6 / 5}+o(X)
$$

This conjecture shows numerical evidence however you can't at the moment prove it. We notice that the constant $c$ is of order $\frac{1}{10}=\frac{1}{2.5}$. To do this we represent, in certain range, the functions

$$
\frac{H(X)}{X^{6 / 5}} \quad \text { and } \quad \frac{\tilde{H}(X)}{X^{6 / 5}} .
$$

Another conjecture out of this observation is that, there exists a positive real $A$ such that for $X>A$ neither $H(X)$ nor $\tilde{H}(X)$ never cross the x-axis. This conjecture is stronger that the previous one. We can the summarize both and ask the following in a more general case: We suppose that $H(X) \sim X^{\theta}$ and the problem here is to give an estimate of $\theta$.

In the appendix we give in section 1, a brief description of the Gauss periods (Lagrange resolvents) and then give a result and conditions for these resolvents to be rational integers. This result is less known though. It is known in general that they are algebraic integers in cyclotomic fields. We give also an elementary proof, due to Cauchy, of the determination of the sign for the quadratic Gauss sums. We end up with an algorithm which computes the Gauss sums and run it for some few values. We add at the last end some data, out of our output file. 



\section{CHAPTER 2}

\section{Generalities on Cyclotomic Fields}

\section{Introduction}

The first investigations of algebraic number fields were made by Gauss, whose study of biquadratic reciprocity led him to introduce the domain $\mathbb{Z}[i]$. Later Eisenstein and Jacobi (not jointly, however), used the domain $\mathbb{Z}[\omega]$ ( $\omega$ is a primitive cubic root of unity) to study the cubic law of reciprocity. In both cases, the domains have the unique factorization property, and in fact, they are what we call Euclidean domains. Later Kummer and Dirichlet tried to use the domain $\mathbb{Z}[\zeta], \zeta$ a primitive root of unity of odd prime degree $\ell$ to show the impossibility of $x^{\ell}+y^{\ell}=z^{\ell}$ in integers. Kummer prepared a proof which rested on the assumption that $\mathbb{Z}[\zeta]$ has unique factorization and submitted it to Dirichlet who pointed out a mistake. This provoked Kummer to invent his theory of ideal numbers which subsequently grew, among others, in the hands of Kronecker and Dedekind into algebraic number theory as we know it. One can refer to $[\mathbf{K}]$. Cyclotomic fields and their subfields, the Abelian fields are thus the first and the most thoroughly studied of the algebraic number fields. This is because Abelian extensions of $\mathbb{Q}$ are the only ones for which the decomposition of primes is determined by their congruence classes to some modulus (called the conductor of the field). This chapter is an exposition of the basic algebraic theory of cyclotomic fields. There are two well known results that will appear throughout the thesis. The first one is that every Abelian extension of $\mathbb{Q}$ is contained in a cyclotomic field and the second one is that if $\mathrm{C}=\mathbb{Q}(\zeta)$ is the "smallest" cyclotomic field containing a field $K$, then the trace $\operatorname{tr}_{\mathrm{C} / \mathrm{K}}(\zeta)$ is a primitive element for $K$. This is a so called Lagrange Resolvent or a Gauss sum.

Let $\Omega$ be an algebraically closed field of characteristic zero. We assume that other fields in this section are contained in $\Omega$. Let $E_{n}=E_{n}(\Omega)$ denote the set of $n^{\text {th }}$ roots of unity contained in $\Omega$ ie: $E_{n}(\Omega)=\left\{a \in \Omega: a^{n}=1\right\} . \quad E_{n}(\Omega)$ is a group of order $n$. If $d \mid n$ then $E_{d} \subseteq E_{n}$ is the unique subgroup of order $d$. Thus $E_{n}$ is a cyclic group of order n. A generator of $E_{n}(\Omega)$ is an element of order $n$. It is a so-called primitive $n^{\text {th }}$ root of unity. The elements of $E_{n}(\Omega)$ are solutions of the polynomial equation $X^{n}-1=0$. In the later steps there is no difference between $\mu_{n}(\Omega)$ and $E_{n}(\Omega)$. If $\Omega=\mathbb{C}$ then $\mu_{n}(\Omega)$ is denoted by $\mu_{n}$. If $d \mid n$, then the polynomial $X^{\frac{n}{d}}-1$ is a factor of $X^{n}-1$. There are $\varphi(n)$ primitive $n^{\text {th }}$ roots of unity. To see this we simply remark that $\zeta_{n}$ and $\zeta_{n}^{m}$ are both primitive root of unity whenever $\operatorname{gcd}(m, n)=1$. Indeed, if $\Phi_{n}(X) \in \mathbb{Z}[X]$ is the minimal polynomial of $\zeta_{n}$, then $\Phi_{n}(X) \mid X^{n}-1$ and it can also be shown that

$$
\Phi(X)=\prod_{\operatorname{gcd}(j, n)=1}\left(X-\zeta_{n}^{j}\right)
$$


However if $\zeta_{n}^{j}$ is another primitive root of unity, then it is a root of the polynomial $X^{n}-1$ but not a root of $\operatorname{gcd}\left(X^{j}-1, X^{n}-1\right)$ and therefore $\Phi_{n}(X)$ can be written as

$$
\Phi_{n}(X)=\frac{X^{n}-1}{\operatorname{gcd}\left(X^{n}-1, \prod_{1 \leq j<n}\left(X^{j}-1\right)\right)}
$$

$\Phi_{n}(X)$ is called the $n^{\text {th }}$ cyclotomic polynomial.

It follows that

$$
X^{n}-1=\prod_{d \mid n} \Phi_{d}(X)
$$

and then the Möbius inversion formula can be applied,

$$
\Phi_{n}(X)=\prod_{d \mid n}\left(X^{n / d}-1\right)^{\mu(d)}
$$

where $\mu(d)$ is the Möbius function.

Definition 2.1. Let $k$ be an arbitrary subfield of $\Omega$. Any extension of $k$ which is of the form $k\left(E_{n}(\Omega)\right)$ is called a cyclotomic field of $k$.

Let $\zeta_{n}$ denote a primitive $n^{\text {th }}$ root of unity. Since the coefficients of the above polynomial belong to the prime field of $\Omega$ and hence to the field $k$, the extension $k\left(\zeta_{n}\right)=k\left(E_{n}(\Omega)\right)$ is a Galois extension of $k$ and its relative degree does not exceed $\varphi(n)$. Let $\mathrm{G}=\operatorname{Gal}\left(k\left(\zeta_{n}\right) / k\right)$ denote the Galois group of the extension $\left.k\left(\zeta_{n}\right) / k\right)$. If $x \in k\left(\zeta_{n}\right) / k$, the action of a Galois element $\sigma \in G$ on $x$ is denoted by $x^{\sigma}$ for $\sigma(x)$. Since for $\sigma \in G$, we have $\zeta^{\sigma} \in E_{n}$, there exits a unique integer $\nu(\sigma) \bmod (n)$, such that $\zeta_{n}^{\sigma}=\zeta_{n}^{\nu(\sigma)}$. This choice of $\nu(\sigma)$ does not depend on the choice of the primitive $n^{t} h$ root of unity $\zeta_{n}$. Furthermore we have

$$
\sigma, \tau \in G, \quad \zeta_{n}^{\sigma \tau}=\left(\zeta_{n}^{\sigma}\right)^{\tau}=\left(\zeta_{n}^{\nu(\tau)}\right)^{\sigma}=\left(\zeta_{n}^{\nu(\sigma)}\right)^{\nu(\tau)}=\zeta_{n}^{\nu(\sigma) \nu(\tau)}
$$

Hence

$$
\nu(\sigma \tau)=\nu(\sigma) \nu(\tau) \bmod n
$$

This signifies that the mapping

$$
\sigma \longmapsto \nu(\sigma) \bmod (n)
$$

is a homomorphism sending $G$ onto the group of residue classes modulo $n$. If $m$ is some integer positive such that

$$
\sigma^{n}\left(\zeta_{n}\right)=\underbrace{\sigma \sigma \cdots \sigma e s}_{n}\left(\zeta_{n}\right)
$$

then

$$
\left(\zeta_{n}\right)^{\sigma^{m}}=\zeta
$$

is equivalent to $m \nu(\sigma) \equiv 1$ modulo $(n)$ which in turn is equivalent to $(\nu(\sigma), n)=1$ for $\sigma \neq 1_{G}$. This is to say that $\nu(\sigma)$ is relatively prime to $n$. The above map is therefore injective and $G$ is an Abelian group, i.e. $k\left(\zeta_{n}\right) / k$ is an Abelian extension. 
Theorem 2.1. Let $k$ be an arbitrary field contained in an algebraically closed field $\Omega$, and let $\zeta_{n}$ be a primitive $n^{\text {th }}$ root of unity in $\Omega(n$ being not divisible by the characteristic of $k)$. Then the extension $k\left(\zeta_{n}\right) / k$ is an Abelian extension and its Galois group Gal $\left(k\left(\zeta_{n}\right) / k\right)$ is sent isomorphically by the mapping $\sigma \rightarrow \nu(\sigma)$, which maps $\zeta_{n}^{\sigma}=\zeta_{n}^{\nu(\sigma)}$, to a subgroup of the group of residue classes of $\mathbb{Z}$ modulo $m$. This isomorphism is given by

$$
\sigma \longmapsto t
$$

and consequently the dimension $[L: k] \leq \varphi(m)$.

Remark Suppose $n, m \in N, n, m \geq 2$ and $(n, m)=1$. Then $\zeta_{n}$ is never a power of $\zeta_{m}$ and vice versa. Therefore, the fields $k\left(\zeta_{n}\right)$ and $k\left(\zeta_{m}\right)$ have as intersection the base field $\mathrm{k}$, and $\zeta_{n m}$ is an element of $k\left(\zeta_{m}, \zeta_{n}\right)$ of order $m n$ i.e. $k\left(\zeta_{m n}\right) \subset k\left(\zeta_{n}, \zeta_{n}\right)$. But $\left(\zeta_{m n}\right)^{n}=\zeta_{m}$ and $\left.\left(\zeta_{m n}\right)^{m}\right)=\zeta_{n}$ implies that $k\left(\zeta_{n}\right)$ and $k\left(\zeta_{m}\right)$ are both contained in $k\left(\zeta_{m n}\right)$ and the minimality of $k\left(\zeta_{m}, \zeta_{n}\right)$ yields the equality .

Note 2.1. From now on, all the fields we use are Galois extensions $K$ of $\mathbb{Q}$.

Definition 2.2. We define for any algebraic integer $a \in K$ :

the norm

$$
N_{K / \mathbb{Q}}(a)=\prod_{\sigma \in G} \sigma(a)
$$

and the trace

$$
\operatorname{Tr}_{K / \mathbb{Q}}(a)=\sum_{\sigma \in G} \sigma(a)
$$

We will sometimes denoted $N_{K / \mathbb{Q}}$ by $N$ and $T r_{K / \mathbb{Q}}$ by $T r$. Both are maps from $K$ to the base field $\mathbb{Q}$.

Definition 2.3. Let $O_{K}$ be the ring of integers of $K, O_{K}^{\times}$, is the group of units of $K$.

\section{Lemma 2.1.}

$$
u \in O_{K}^{\times} \Longleftrightarrow N_{K / \mathbb{Q}}(u)= \pm 1
$$

In the following steps, we shall denote the ring of integers of $K, \mathbb{Z}\left[\zeta_{n}\right]^{\times}$, by $O_{K}$ and the group of units of $K, O_{K}^{\times}$, is sometimes denotes by $\mathrm{U}(\mathrm{K})$.

Definition 2.4. The discriminant $\Delta=\Delta(K / \mathbb{Q})$ of the cyclotomic field $K=\mathbb{Q}\left(\zeta_{n}\right)$ is defined to be $\Delta(\zeta)=\prod_{j \neq i}\left(\zeta^{i}-\zeta^{j}\right)$ where $\zeta=\zeta_{n}$ is any primitive root of unity.

$$
\Delta(K)=(-1)^{n(n-1) / 2} \prod_{j \neq i}\left(\zeta^{i}-\zeta^{j}\right)
$$


Definition 2.5. The $O_{K}$-module generated by

$$
\left\{\alpha: \alpha \in K, \operatorname{Tr}_{K / \mathbb{Q}}\left(\alpha O_{K}\right) \subset \mathbb{Z}\right\}
$$

is a fractional ideal $\mathfrak{M}(K / \mathbb{Q})$ containing $\mathbb{Z}$. The different $\mathfrak{D}=\mathfrak{D}(K / \mathbb{Q})$ of the extension field $K=\mathbb{Q}\left(\zeta_{n}\right)$ over $\mathbb{Q}$ is defined to be the inverse of the $\mathbb{Z}$-module $\mathfrak{M}(K / \mathbb{Q})$ :

$$
\mathfrak{D}=\mathfrak{M}(K / \mathbb{Q})^{-1}
$$

Remark If we replace $\mathbb{Q}$ by $\mathbb{Q}_{p}$, then $K$ is a local field with maximal prime ideal $\mathfrak{P}$ then

$$
\mathfrak{D}=\mathfrak{P}^{N}
$$

for some positive integer $N$.

\section{Remark}

$$
\Phi_{n}\left(\zeta^{a}\right) \mid n^{n} \text { for all integers } a, \quad \text { with } \operatorname{gcd}(a, n)=1 \text {. }
$$

The discriminant

$$
\Delta=\delta\left(1, \zeta, \cdots, \zeta^{\phi(n)-1}\right) \mid n^{n}
$$

Remark If $n=p$ is prime, then the Galois group $G=\operatorname{Gal}(K / \mathbb{Q})$ of $K / \mathbb{Q}$ is of order $p-1$ and therefore 2 divides $|G|$. Therefore by the elementary theory of finite groups and the Galois connection between subfields of $K$ and subgroups of $G$, this assures that $\mathrm{K}$ contains a quadratic field $K_{1}$. Since the Galois group is cyclic, then $G=\langle\sigma\rangle$ for some generator $\langle\sigma\rangle$. $K_{1} / \mathbb{Q}$ is of degree 2 and $K / K_{1}$ is of degree $\frac{p-1}{2}$. We will see later that this field is rather easily constructed by using the Gauss sums. This in fact always assures the existence of a quadratic field in a prime power cyclotomic field. This even extends to any cyclotomic field, since the function $\phi(n)$ is even for $n>2$.

Let $t$ denote the degree of $K / \mathbb{Q}$. Then the homomorphism

$$
\varphi: K \rightarrow K \otimes_{\mathbb{Q}} \mathbb{R}, \quad x \mapsto x \otimes 1
$$

is an injective $\mathrm{K}$-homomorphism. The $\mathbb{R}$-algebra $K \otimes_{\mathbb{Q}} \mathbb{R}$ decomposes as $K \otimes_{\mathbb{Q}} \mathbb{R} \cong$ $\mathbb{R}^{r} \times \mathbb{C}^{s}$, where $(r, s)$ is called the signature of the field $\mathrm{K}$. In general the component map

$$
\varphi_{i}: K \rightarrow \mathbb{R} \quad \text { or } \quad \mathbb{C}
$$

is called real or complex embedding if its range is $\mathbb{R}$ or $\mathbb{C}$ respectively. That is, $\varphi$ is written component wise $\left(\varphi_{i}\right) 1 \leq i \leq r+s$ such that the $r$ real first components are real embeddings and the $s$ second components are complex embeddings up to complex conjugacy.

1.1. Decomposition of primes. Let us suppose, as before, that $\mathrm{K}$ is an algebraic number field, Galois over $\mathbb{Q}$ of finite degree, and $G=G(K / \mathbb{Q})$ is its Galois group. Denote $O_{K}$ the ring of integers of $\mathrm{K}$.

Any prime integral ideal $\mathfrak{P}$ of $K$ intersects $\mathbb{Z}$ at some rational prime $p$ and we say that $\mathfrak{P}$ is above $(p)$. This also means $\mathfrak{P} \mid p$ and therefore the norm $N(\mathfrak{P}) \mid N(p)$ implies that $|N(\mathfrak{P})|=p^{f}$ for some $f \leq n . f=f_{\mathfrak{P}}$ is called the residue class degree of $\mathfrak{P} / p$. However any other conjugate of a prime ideal above $\mathfrak{P}$ is still a prime above $p$ since 
the Galois isomorphism maps primes onto primes and leaves invariant the ideals of the base field. In general, not all the ideals $\sigma(\mathfrak{P}), \sigma \in G$ are distinct. However we are in a situation of a unique factorization domain. Thus, to each prime ideal $\mathfrak{P}$, we associate the Galois subgroup

$$
G(\mathfrak{P})=\{\sigma \in G: \sigma(\mathfrak{P})=\mathfrak{P}\} .
$$

$G(\mathfrak{P})$ is called the decomposition group of $\mathfrak{P}$. The corresponding subfield to this subgroup is called the decomposition field of $\mathfrak{P}$. The order $e_{\mathfrak{P}}$ of $G(\mathfrak{P})$ is the exponent of $\mathfrak{P}$ in the factorization of $p$. It is called the ramification degree of $\mathfrak{P} / p$. From the transitivity of the Galois group we see that all the $e_{\sigma(\mathfrak{P})}$ are equal, and we denote it by $e$. Let $r$ be the number of distinct primes above $p$, we thus have $r e f=n$. The following theorem describes the action of the Galois group on the decomposition:

Theorem 2.2. Let $K / \mathbb{Q}$ be a Galois extension. Let $p \in \mathbb{Z}$ be a prime number and write $(p)=\mathfrak{P}_{1}^{e_{1}} \cdots \mathfrak{P}_{r}^{e_{r}}$. Then $e=e_{1}=\cdots=e_{r}$ and $f=f_{1}=\cdots=f_{r}$ so that efr $=n$.

ProOF. See [I.R], pp $181-183$

Definition 2.6. If $e(\mathfrak{P} / p)>1$ we say that the prime ideal $\mathfrak{P}$ ramifies in $K / \mathbb{Q}$. i.e $(p) \subset \mathfrak{P}^{2}$.

Let $p, \quad \mathfrak{P} \mid p$ be as before, then $\kappa=O_{K} / \mathfrak{P}$ is a finite field with $N(\mathfrak{P})$ elements. It is the residue class field at $\mathfrak{P}$ and it contains $\mathbb{F}_{p}=\mathbb{Z} /(p)$ as a subfield. It is also a Galois extension of $\mathbb{Z} /(p)$ with Galois group $\bar{G}=\operatorname{Gal}\left(\kappa / \mathbb{F}_{p}\right)$, if we define the homomorphic map from $G(\mathfrak{P})$ to $G a l\left(\kappa / \mathbb{F}_{p}\right)=\bar{G}$ by $\bar{\sigma}(\alpha(\bmod \mathfrak{P}))=\sigma(\alpha)$ $(\bmod \mathfrak{P})$. The map $\sigma \mapsto \bar{\sigma}$ is a well defined map and is onto. Let

$$
T(\mathfrak{P})=\{\sigma \in G: \sigma(\alpha) \equiv \alpha \bmod (\mathfrak{P}) \text { for all } \alpha \in K\}
$$

is the kernel of this homomorphism, then $G(\mathfrak{P}) / T(\mathfrak{P}) \cong \bar{G} . T(\mathfrak{P})$ is called the inertia group of $\mathfrak{P}$ and the corresponding field is called the inertia field of $\mathfrak{P}$.

We notice that the order of $G(\mathfrak{P}) / T(\mathfrak{P})$ is $f$ and is also a cyclic group of the same order, generated by the homomorphism $\bar{\alpha} \mapsto \bar{\alpha}^{p}, \alpha \in O_{K}$. The element of the quotient group $G(\mathfrak{P}) / T(\mathfrak{P})$ associated by the above isomorphism to the latter operation is called a Frobenius class associated with $(K / \mathbb{Q}, \mathfrak{P})$ and is denoted by $\sigma_{p}$.

Lemma 2.2. Let $p$ be a prime integer such that $p \not \mid n$.

Then $\sigma_{p}$ is the element of the Galois group $G(K / \mathbb{Q})$ which maps $\zeta \mapsto \zeta^{p}$ The equation $\zeta^{a} \equiv \zeta^{b}(\bmod p)$ implies that $\zeta^{a}=\zeta^{b}$

ProOF. $\sigma_{p}(\zeta)=\zeta^{a}$ for some $a \in \mathbb{Z}$ with $\operatorname{gcd}(a, n)=1$ $\sigma_{p}(\zeta) \equiv \zeta^{p} \bmod ((p))$ implies that $p \mid\left(1-\zeta^{j}\right)$ for some $j, 0<j<n$ and therefore $p \mid n$ which is a contradiction of the assumption.

Remark The order of the Frobenius element $\sigma_{p}$ is $f$. Thus an equation of the form $\sigma_{p}^{m}=i d \in \operatorname{Gal}(K / \mathbb{Q})$ is equivalent to saying that $\sigma_{p}^{m}(\zeta)=\zeta$. This implies $p^{m} \equiv 1 \bmod (n)$, therefore $f$ is the smallest positive integer such that $p^{f} \equiv 1 \bmod (n)$ Moreover if $\sigma_{p_{1}}=\sigma_{p_{2}}$ then $\zeta^{p_{1}}=\zeta^{p_{2}}$ which is equivalent to $n \mid\left(p_{1}-p_{2}\right)$. Thus, 
two primes in the same arithmetic progression with difference $n$ have the same decomposition law. The converse is false. To see this take $K=\mathbb{Q}\left(\zeta_{8}\right)$ and prime $p \equiv 3,5,7 \bmod (8)$, which have the same law of decomposition.

Now suppose that $n$ is a power of a prime $p$, say, $m=p^{s}$. Then every primitive $p^{s} t h$ root of unity $\zeta$, is a root of $X^{p^{s}}-1$ but not that of $X^{p^{s-1}}-1$. We have, therefore,

$$
\prod_{\left(i, p^{s}\right)=1}\left(X-\zeta^{i}\right)=\frac{X^{p^{s}}-1}{X^{p^{s-1}}-1}=X^{p^{(s-1)(p-1)}}+\cdots+X^{p^{(s-1)}}+1
$$

and in particular

$$
p=\prod_{\left(i, p^{s}\right)=1}\left(1-\zeta^{i}\right)
$$

For each such $i$,

$$
\frac{1-\zeta^{i}}{1-\zeta}=1+\zeta+\cdots+\zeta^{i-1}
$$

belongs to $O_{K}$. Moreover there exists $j$ such that $j i \equiv 1 \bmod \left(p^{s}\right)$ and $0<j<p^{s}$ thus,

$$
\frac{1-\zeta}{1-\zeta^{i}}=\frac{1-\zeta^{i j}}{1-\zeta^{i}}=1+\zeta^{i}+\cdots+\zeta^{i j-1} \in O_{K}
$$

Therefore $1-\zeta^{i}$ and $1-\zeta$ are associates in $O_{K}$ and we may write

$$
p=u(1-\zeta)^{\phi\left(p^{s}\right)}
$$

where $u$ is a unit and $\phi\left(p^{s}\right)$ is the Euler phi function.

The prime $\lambda=(1-\zeta)$ is above $p$ and because of $r=1$ the equation ref $=\phi\left(p^{s}\right)$ yields $f=1$ and $e=\phi\left(p^{s}\right)$.

Theorem 2.3. If $p \mid m$ and we write $n=p^{s} m$ with $\operatorname{gcd}(p, m)=1$, then $p$ factors in $O_{K}$ in the form

$$
(p)=\left(\mathfrak{P}_{1} \cdots \mathfrak{P}_{r}\right)^{\phi\left(p^{s}\right)}
$$

where $\mathfrak{P}_{1} \cdots \mathfrak{P}_{r}$ are distinct prime ideals of $O_{K}$ of degree $f, f r=\phi(m)$ and $f$ is the smallest positive integer such that $p^{f} \equiv 1 \bmod (m)$.

Proof. This is a double application of both cases where $p \nmid \not m$ follows by $p \mid p^{s}$.

We give an explicit factorization of the primes. This method is due to Dedekind. See $[\mathbf{S . L}]$ pp 27-28.

\section{Proposition 1.1. Explicit factorization of primes}

Let $A$ be a Dedekind domain with quotient field $K$. Let $E$ be a finite separable extension of $K$. Let $B$ be the integral closure of $A$ in $E$ and assume that $B=A[\alpha]$ for some element $\alpha$. Let $f(X)$ be the irreducible polynomial of $\alpha$ over $K$. Let $\mathfrak{p}$ be a prime of $A$. Let $\bar{f}$ be the reduction of $f$ and $\mathfrak{p}$, and let

$$
\bar{f}(X)=\bar{P}_{1}(X)^{e_{1}} \cdots \bar{P}_{r}(X)^{e_{r}}
$$

be the factorization of $\bar{f}$ into powers of irreducible factors over $\bar{A}=A / \mathfrak{p}$ with leading coefficient 1. then

$$
\mathfrak{p} B=\mathfrak{P}_{1}^{e_{1}} \cdots \mathfrak{P}_{r}^{e_{r}}
$$


is the factorization of $\mathfrak{p}$ in $B$, so that $e_{i}$ is the ramification index of $\mathfrak{P}_{i}$ over $\mathfrak{p}$, and we have

$$
\mathfrak{P}_{i}=\mathfrak{p} B+P_{i}(\alpha) B,
$$

if $P_{i}(X) \in A[X]$ is a polynomial with leading coefficient 1 whose reduction mod $\mathfrak{p}$ is $\overline{P_{i}}$.

The main theorem of the unit group is given by,

Theorem 2.4. The Dirichlet Unit Theorem.

Let $(r, s)$ be the signature of $K$ then the group of units is a finitely generated Abelian group of rank $r+s-1$. In other words, we have a group isomorphism

$$
U(K) \cong \mu(K) \times \mathbb{Z}^{r+s-1}
$$

and $\mu(K)$ is the finite cyclic group of the roots of unity in $K$.

Proof. See $[\mathbf{P . S}]$, and [Min].

Let first define $\varepsilon_{1}, \cdots, \varepsilon_{t}$ to be a basis of $\mathbb{Z}^{r+s-1}$, a unit $u$ of $\mathrm{K}$ can be written in a unique way as

$$
u= \pm \zeta^{i} \varepsilon_{1}^{n_{1}} \cdots \varepsilon_{t}^{n_{t}}
$$

where $\zeta$ is root of unity, $1 \leq i \leq n, n_{i} \in \mathbb{Z}$. Such a family $\left(\varepsilon_{i}\right)$ will be called a system of fundamental units of $K$. It is not unique though, since the changing of a $\mathbb{Z}$-basis of $\mathbb{Z}^{r+s-1}$ results in another basis by a matrix of determinant +1 or -1 . It is however possible to have the $\left(\varepsilon_{i}\right)$ real positive greater than 1 , and in this case we have a system of real fundamental units.

Remark If $K$ a cyclotomic field, it has even degree over $\mathbb{Q}$, and $r=0$ and $s=\phi(n) / 2$. If $n$ is even then $\# \mu(K)=n$ otherwise $\# \mu(K)=2 n$

Remark The group of units of $K=\mathbb{Q}\left(\zeta_{n}\right)$ is a finitely generated Abelian group of rank $\frac{\phi(n)}{2}-1 . \mu(K)$ is the finite cyclic group of the roots of unity in K.

Let $n>2$ be a prime. Though the existence of a system of $\frac{n-3}{2}$ fundamental units for cyclotomic fields is due to Dirichlet, they have been constructed explicitely and independently by Kronecker and Kummer. If $n>2$, prime as before, then $N_{K / \mathbb{Q}}\left(\zeta^{i}+\zeta^{-i}\right)= \pm 1$, for $i \neq 0$

Put

$$
\varepsilon_{i}=\zeta^{i}+\zeta^{-i} \quad \text { for } \quad 1 \leq i \leq \frac{n-3}{2}
$$

and apply the above lemma.

Let the power symbol $\bar{x}$ denote the complex conjugate in $\mathbb{C}$. This is an automorphism. It is of order 2, so that the corresponding subfield $K_{1}$ is such that $K / K_{1}$ is of degree 2 and consequently $K_{1} / \mathbb{Q}$ is of degree $\phi(n) / 2$. The field $\mathbb{Q}\left(\zeta+\zeta^{-1}\right)$ is a real field which is pointwise fixed under complex conjugate automorphism so that $\mathbb{Q}\left(\zeta+\zeta^{-1}\right) \subset K_{1}$. Since $\zeta$ is a root of the polynomial $X^{2}-\left(\zeta+\zeta^{-1}\right) X+1$ we have $K_{1}=\mathbb{Q}\left(\zeta+\zeta^{-1}\right)$.

$K_{1}$ is called the maximal real subfield of $K$ and it will be denoted by $K^{+}$. 
For $\mathbf{n}=\mathbf{5}$, the real (quadratic) subfield is $K_{1}=\mathbb{Q}\left(\zeta+\zeta^{-1}\right)$ and from the equation

$$
\zeta+\zeta^{2}+\zeta^{3}+\zeta^{4}+1=0,
$$

a unit has the form $\zeta^{i} \varepsilon_{1}^{k}=u_{k}^{i}$. We can fix the complex embedding $\varphi$ such that

$$
\tau=\varphi\left(-\zeta^{2}-\zeta^{3}\right)=\frac{1+\sqrt{5}}{2}>1 .
$$

If $p$ is an odd prime and $g$ a primitive root modulo $p$, a classical result of Hilbert, [Hilbert Satz 142], says that:

$$
\left(\frac{\left(1-\zeta^{g^{k+1}}\right)\left(1-\zeta^{-g^{k+1}}\right)}{\left(1-\zeta^{g^{k}}\right)\left(1-\zeta^{-g^{k}}\right)}\right)^{\frac{1}{2}}
$$

form, for $k=0,1,2, \cdots, \frac{p-3}{2}$, a system of fundamental units in the field $\mathbb{Q}\left(\zeta_{p}\right)$. For $p$ not exceeding 161, these units form a system of fundamental units. This is no longer true for $p=163$. A system of fundamental units related to the above formula was given by H. Vandivier [V]. J. Milnor conjectured that if $K=\mathbb{Q}\left(\zeta_{n}\right)$ with $\varphi(n)>2$, then the units

$$
\frac{\zeta_{n}^{s}-1}{\zeta_{n}-1}, \quad 2 \leq s<n / 2
$$

form a system of fundamental units for $K$. This conjecture was disproved by Ramachandra $[\mathbf{R a m}]$ who showed that if $n$ has at least two distinct odd prime divisors, then the units from the last formula are multiplicatively dependent. However, if $n$ is prime they are independent and then form together with $\zeta_{n}$ a subgroup of finite index in the group $U(K)$. Similar results were obtained by H. Bass, $[\mathbf{B a}]$

\section{Power residue Symbol}

We give a definition of the power residue symbol and basic properties that are of use in the definition and computation of Gauss sums. This will be defined in a general situation as possible.

Let $k$ be a number field of finite degree containing $\zeta_{n}$, a primitive $n^{\text {th }}$ root of unity. Let $O_{k}$ be the ring of integers of $k$. A prime ideal $\mathfrak{p}$ in $O_{k}$ is said to be prime to $n$ if and only if $q=N(\mathfrak{p})$ is such that $(q, n)=1$. The residue class field at $\mathfrak{p}, \kappa=O_{k} / \mathfrak{p}$ is finite of order $q$. Let $\alpha, \mathfrak{p} \in \mathbb{Z}\left[\zeta_{n}\right]$, where $\mathfrak{p}$ is prime with $\mathfrak{p} \backslash \alpha$. Then $\mathbb{Z}\left[\zeta_{n}\right] /(\mathfrak{p})$ is a field with $N(\mathfrak{p})$ elements. Therefore, the analogue of the little Fermat theorem says:

$$
\alpha^{N(\mathfrak{p})-1} \equiv 1 \quad(\bmod \mathfrak{p}) \quad \text { and } \quad \mathfrak{p} \not \alpha .
$$

In the following steps we denote $\zeta_{n}$ simply by $\zeta$.

Proposition 2.1. Let $\mathfrak{p}, \alpha \in \mathbb{Z}[\zeta]$ with $\mathfrak{p}$ prime, and $\operatorname{gcd}(N(\mathfrak{p}), n)=1$ and $\mathfrak{p} \backslash \alpha$. Then there exists a unique integer $m, 0 \leq m \leq n-1$, such that

$$
\alpha^{(N(\mathfrak{p})-1) / n} \equiv \zeta^{m} \quad(\bmod \mathfrak{p}) .
$$


Proof. Indeed, for all primes $\mathfrak{p} \in \mathbb{Z}[\zeta]$ with $\operatorname{gcd}(N(\mathfrak{p}), n)=1$ we have

$$
n \mid(N(\mathfrak{p})-1) .
$$

Hence, we can write

$$
\alpha^{N(\mathfrak{p})-1}-1=\prod_{i=0}^{n}\left(\alpha^{(N(\mathfrak{p})-1) / n}-\zeta^{i}\right) .
$$

But $\mathfrak{p} \mid\left(\alpha^{N(\mathfrak{p})-1}-1\right)$ hence $\mathfrak{p}$ divides at least one of the factors on the right hand side. Suppose $\mathfrak{p}$ divides at least two of them, say

$$
\left(\alpha^{(N(\mathfrak{p})-1) / n}-\zeta^{i}\right) \text { and }\left(\alpha^{(N(\mathfrak{p})-1) / n}-\zeta^{j}\right) \text { with } 0 \leq i<j<n .
$$

Then $\mathfrak{p} \mid \zeta^{i}\left(1-\zeta^{j-i}\right)$, and thus $N(\mathfrak{p}) \mid N\left(1-\zeta^{j-i}\right)$, since $\mathfrak{p}$ is not a unit and $\zeta$ primitive implies that $N\left(1-\zeta^{j-i}\right)=n$ or \pm 1 , we deduce that $\operatorname{gcd}(N(\mathfrak{p}), n) \neq 1$ which contradicts the assumption. Therefore, there exists a unique power $m$ such that

$$
\alpha^{(N(\mathfrak{p})-1) / n} \equiv \zeta^{m} \bmod (\mathfrak{p}) .
$$

Hence we can define the $n^{\text {th }}$ residue symbol $\left(\frac{\alpha}{\mathfrak{p}}\right)_{n}$ to be the unique $n^{\text {th }}$ root of unity such that

$$
\alpha^{\frac{q-1}{n}} \equiv\left(\frac{\alpha}{\mathfrak{p}}\right)_{n} \bmod \mathfrak{p}
$$

whenever $(\alpha, \mathfrak{p})=(1)$

We extend $\left(\frac{\alpha}{\mathfrak{p}}\right)_{n}$ as a multiplicative function of the ideals $\mathfrak{p}$ relatively prime to $n$ i.e if $\mathfrak{a}=\prod \mathfrak{p}$ for such ideals $\mathfrak{p}$ then

$$
\left(\frac{\alpha}{\mathfrak{a}}\right)_{n}=\prod\left(\frac{\alpha}{\mathfrak{p}}\right)_{n} .
$$

Definition 2.7. For $\mathfrak{a}$ such that $(\mathfrak{a}, n)=1,\left(\frac{\alpha}{\mathfrak{a}}\right)_{n}$ is well defined and is called the $n^{\text {th }}$ power residue symbol.

\section{Remark}

- The residue symbol is a function of both arguments whenever it is defined.

- From the definition we see that if $\alpha$ is an $n^{\text {th }}$ power, then $\left(\frac{\alpha}{\mathfrak{a}}\right)_{n}=1$. This shows that, if $\alpha$ and $\beta$ differ by a factor which is an $n^{\text {th }}$ power and satisfy the necessary condition with $\mathfrak{p}$, then $\left(\frac{\alpha}{\mathfrak{p}}\right)_{n}=\left(\frac{\beta}{\mathfrak{p}}\right)_{n}$

In order to make this definition more general without any restriction to $(\alpha)+\mathfrak{a}=(1)$, we shall extend the definition to $\alpha$ such that

$$
(\alpha)+\mathfrak{a} \neq(1) \text { by }\left(\frac{\alpha}{\mathfrak{a}}\right)_{n}=0
$$

Consider furthermore $K / k$ any other finite extension of the number field $k$. We assume $k$ still contains the $n^{\text {th }}$ roots of unity, then the $n^{\text {th }}$ power residue symbol is 
also a function depending upon the underlined field. The notation becomes $(\div)_{n, F}$, where $F$ is the underlying field. If $K / k$ is a normal extension, then the Galois group $\operatorname{Gal}(\mathrm{K} / \mathrm{k})$ acts on the power residue symbol $(\dot{*})_{n, k}$ and $(\dot{\xi})_{n, K}$. As we see it in the next proposition

Proposition 2.2. Let $K / k$ be a normal extension with Galois group $G$, and let $k / F$ be a subextension such that a primitive $n^{\text {th }}$ root of unity $\zeta_{n} \in k$.

1. For every $\sigma \in G$, we have $\left(\frac{\alpha}{\mathfrak{a}}\right)_{n, K}^{\sigma}=\left(\frac{\alpha^{\sigma}}{\mathfrak{a}^{\sigma}}\right)_{n, K}$, for all $\alpha \in K^{*}$ and all ideals $\mathfrak{a}$ prime to $n$.

2. if $\mathfrak{p}$ has inertia degree 1 in $K / k$, then $\left(\frac{\alpha}{\mathfrak{P}}\right)_{n, K}^{\sigma}=\left(\frac{\alpha}{\mathfrak{p}}\right)_{n, K}^{\sigma}$, where $\mathfrak{P}$ is a prime ideal in $O_{K}$ above $\mathfrak{p}$ and $\alpha \in O_{k}$.

3. If $K / k$ is Abelian and $\mathfrak{p}$ is a prime ideal in $O_{k}$, then $\left(\frac{\alpha}{\mathfrak{p}}\right)_{n, K}=\left(\frac{\alpha}{N \mathfrak{p}}\right)_{n, k}$, for all $\alpha \in O_{K}$, where $N=N_{K / k}$ denotes the relative norm.

4. Let $K / k$ be a cyclic extension of degree $(K: k)=n$, and suppose that there is a prime ideal $\mathfrak{p} \chi_{n} O_{k}$ such that $\mathfrak{p}=\mathfrak{P}^{n}$ is totally ramified in $K / k$. Then $\left(\frac{N_{K / k} \alpha}{\mathfrak{p}}\right)_{n, k}=1$ for all $\alpha \in O_{K}-\mathfrak{P}$.

ProOF. See [F-Lem] pp 112.

\section{The primary choice of the prime factor in $\mathbb{Q}\left(\zeta_{5}\right)$}

In this small section, we explain the construction of primality factor for prime in the cyclotomic field $\mathbb{Q}\left(\zeta_{5}\right)$. This, in fact turn out to be a general pattern. We will be using this kind of prime factors troughout the dissertation. We specialize the definition as follows:

Definition 2.8. Let $\pi \mid p$ be a prime above $\mathrm{p}$, then we say that $\pi$ is primary if

$$
\pi \equiv 1 \bmod (1-\zeta)^{3} \text {. }
$$

Let $\zeta$ be a primitive fifth root of unity in $K=\mathbb{Q}\left(\zeta_{5}\right)$. Then

$$
\mathbb{Q} \subset \mathbb{Q}(\sqrt{5}) \subset K,
$$

and $\left(\zeta-\zeta^{2}-\zeta^{3}+\zeta^{4}\right)^{2}=5$, therefore we can set $\sqrt{(5)}=\left(\zeta-\zeta^{2}-\zeta^{3}+\zeta^{4}\right)$. The ring of integer $O_{K}$ is a unique factorization domain and its units are all of the form $\pm \zeta^{i} \tau^{j}$, for some integers $i, j$ and where $\tau=-\zeta^{2}-\zeta^{3}$, is a fundamental unit in $\mathbb{Q}(\sqrt{5})$. If $\pi$ is a prime of $O_{K}$, then $O_{\pi}$ is the completion of $O_{K}$ at $\pi$ and $K_{\pi}$ the respective fraction field. If we let $\lambda=1-\zeta$, thus $\lambda$ generates the sole prime of $O_{K}$ above 5 . Therefore the $\lambda$-adic expansion of $\tau$ shows that

$$
\tau \equiv-2 \bmod \lambda
$$

and

$$
\tau^{2} \equiv-1+\lambda^{2} \bmod \lambda^{3}
$$

We have the following lemma

Lemma 2.3. $\quad 1$ If $\alpha$ is an algebraic integer prime to $\lambda$, then there is an associate $\alpha^{*}$ of $\alpha$ such that

$$
\alpha^{*} \equiv 1 \bmod \lambda^{3}
$$


- 2 If $\alpha_{1}^{*}$ and $\alpha_{2}^{*}$ are two associates congruent to 1 modulo $\lambda^{3}$, then their ratio is a fifth power of a unit in $O_{K}$.

- 3 Let $n \in \mathbb{Z}$ be prime to 5 . Then any associate $n^{*}$ of $n$ with

$$
n^{*} \equiv 1 \bmod \lambda^{3}
$$

is $n$ times a fifth power of a unity in $O_{K}$.

Proof. $\quad 1$ - Let $\alpha \in \mathbb{Z}[\zeta]$, the $\lambda$-adic expansion of $\alpha$ shows that $\alpha \equiv$ $n \bmod \lambda, n \in \mathbb{Z} /(5)$ and $\lambda$ prime to $\alpha$ implies that $n \not \equiv 0 \bmod 5$, thus we can assume $n>0$. Since $\tau$ is a primitive root modulo 5 , there exists $j, \quad 0 \leq j<4$ such that

$$
\tau^{j} n \equiv 1 \bmod \lambda
$$

Moreover $\tau^{2} \equiv-1 \bmod \lambda$ and so, there exist $0 \leq i, j \leq 1$ so that

$$
(-1)^{i} \tau^{j} \alpha \equiv 1 \bmod \lambda \text {. }
$$

Then

$$
(-1)^{i} \tau^{j} \alpha \equiv 1+m \lambda \bmod \lambda^{2}
$$

and we can assume $0 \leq m \leq 4$. From this restriction follows that

$$
\zeta^{m}(-1)^{i} \tau^{j} \alpha \equiv 1 \bmod \lambda^{2} .
$$

Let

$$
\zeta^{m}(-1)^{i} \tau^{j} \alpha \equiv 1+\ell \lambda^{2} \bmod \lambda^{3}
$$

Since $-\tau^{2} \equiv 1-\lambda^{2}$, we deduce that

$$
(-\tau)^{2 \ell} \zeta^{m}(-1)^{i} \tau^{j} \alpha \equiv 1 \bmod \lambda^{3},
$$

for $0 \leq \ell \leq 4$, i.e

$$
(-1) i+\ell \zeta^{m}(-1)^{i} \tau^{j+2 \ell} \alpha \equiv 1 \bmod \lambda^{3} .
$$

- 2- If $\lambda_{1}^{*}$ and $\lambda_{2}^{*}$ are two such associates, then their ratio is a unit. Since both $\lambda_{1}^{*}$ and $\lambda_{2}^{*}$ are congruent to 1 modulo $\lambda^{3}$ their ratio

$$
u=\frac{\lambda_{1}^{*}}{\lambda_{2}^{*}}
$$

is also congruent to $1 \bmod \lambda^{3}$.

Since $-\tau^{10} \equiv 1 \bmod \lambda^{3}$ i.e $\left(-\tau^{2}\right)^{5} \equiv 1 \bmod \lambda^{3}$, we consider

$$
u=(-1)^{i} \tau^{j} \zeta^{k}\left(-\tau^{2}\right)^{l}\left(-\tau^{10}\right)^{m}
$$

modulo $\lambda, \lambda^{2}$ yields (by 1 ) $i=j=0$ and $k=l=0$ since

$$
0 \leq i, j, k, l \leq 4
$$

$u$ is then a tenth power of a unit and a fortiori a fifth power.

- 3- Since $n^{4} \equiv 1 \bmod 5$, and $n^{* 4} \equiv 1 \bmod \lambda^{3}$ is an associate of $n^{4}$, by 2 ), $\left(\frac{n}{n^{*}}\right)^{4}$ is a fifth power of a unit, so dividing by $\left(\frac{n}{n^{*}}\right)^{5}$, shows that $\frac{n}{n^{*}}$ is a fifth power of a unit and therefore 3 falls. 
The ring $\mathbb{Z}[\zeta]$ is a unique factorization domain and $K^{+}=\mathbb{Q}(\sqrt{5})$. Thus every prime $p \equiv 1 \bmod 5$ is the norm of some prime $\pi \in O_{K}$, and $4 p=a^{2}-5 b^{2}$ for some rational integers, $a, b$.

Lemma 2.4. If $4 p=a^{2}-5 b^{2}$, with $a, b$ in $\mathbb{Z}$, and if $\pi \in O_{K}$ is a prime divisor of

$$
x=\frac{a+b \sqrt{5}}{2} \quad \text { in } \quad O_{K}
$$

then there exists an associate of $\pi$ in $O_{K}$ whose norm in $K / K^{+}$is $\pm x$.

Proof. The norm of $x \in K^{+} / \mathbb{Q}$ is $p$, which splits as a product of 4 primes in $\mathbb{Z}[\zeta]$. Since $\mathbb{Z}[\zeta]$ is a unique factorization domain, $x$ is, to within a unique factor $u \in K^{+}$, the product of a prime $\pi$ and its conjugate $\bar{\pi}$. Thus $x=u N_{K / K^{+}}(\pi)$, and upon taking the norm of both sides in $K^{+} / \mathbb{Q}$, one gets $N_{K^{+} / \mathbb{Q}}(\gamma)=1$. Since $K^{+}$ is a quadratic field in which a generator of the unit group is $\gamma=\zeta+\zeta^{-1}$, one has $u= \pm \gamma^{k}$ with $k \in \mathbb{Z}$. Also, since $N_{K^{+} / \mathbb{Q}}(u)=-1$, it follows that $k=2 j$ for some integer $j$. Noting that $\gamma^{2}=\mathrm{N}_{K / K^{+}}\left(1-\zeta^{2}\right)$, it follows that $x= \pm N_{K / K^{+}}\left(\left(1-\zeta^{2}\right)^{j} \pi\right)$, and hence the result. 


\section{CHAPTER 3}

\section{GAUSS SUMS}

\section{Gauss sums in number fields}

Let $I$ be a non zero ideal in the ring of integers $O_{K}$ and define $\mathfrak{D}(K / \mathbb{Q})$ the different of $O_{K}$. The trace of $\alpha \in K$ is

$$
\operatorname{Tr}_{K / \mathbb{Q}}(\alpha)=\sum_{\sigma \in \operatorname{Gal}(K / \mathbb{Q})} \sigma(\alpha)
$$

This is rational integer if $\alpha \mathfrak{D}(K / \mathbb{Q})$ is an integral ideal.

Let $a$ be a fixed number lying in the fractional ideal $(I \mathfrak{D}(K / \mathbb{Q}))^{-1}$. If $\chi$ is any character of $G[I]$, the set of invertible elements modulo $I$, then the Gauss sum corresponding to the pair $(\chi, a)$ is:

$$
G(\chi, a)=\sum_{x \bmod I} \chi(x) \exp \left(2 \pi i \operatorname{Tr}_{K / \mathbb{Q}}(a x)\right) .
$$

Proposition 1.1. The sum

$$
\sum_{x \bmod I} \chi(x) \exp \left(2 \pi i \operatorname{Tr}_{K / \mathbb{Q}}(a x)\right)
$$

does not depend on the residue classes modulo $I$.

Proof. Let $X$ and $Y$ be two sets of residues modulo $I$, then for all $x \in X$ and all $y \in Y$, if $x$ and $y$ are such that $x \equiv y \bmod I, x, y \in O_{K}$ then $\chi(x)=\chi(y)$. But we can write $a x=a y+a(x-y)$, such that $\operatorname{Tr}_{K / \mathbb{Q}}(a x)=\operatorname{Tr}_{K / \mathbb{Q}}(a y)+\operatorname{Tr}_{K / \mathbb{Q}}(a(x-y))$. But $a(x-y) \in \mathfrak{D}(K / \mathbb{Q})^{-1}$ therefore we get $\operatorname{Tr}_{K / \mathbb{Q}}(a(x-y)) \in \mathbb{Z}$.

Thus $\exp \left(2 \pi i \operatorname{Tr}_{K / \mathbb{Q}}(a x)\right)=\exp \left(2 \pi i \operatorname{Tr}_{K / \mathbb{Q}}(a y)\right)$.

\section{Remark}

- For $K=\mathbb{Q}$ then $I$ is a principal ideal generated by some $n \in \mathbb{Z}$. If $k \in \mathbb{Z}$ and $a \in \mathbb{Q}$ are such that $k=a n$, the we denote this sum by

$$
\tau_{k}(\chi)=\sum_{x \bmod n} \chi(x) \exp (2 \pi i k x / n) .
$$

We denote $\tau_{1}(\chi)$ simply by $\tau(\chi)$.

- If $a \equiv b \bmod I$ then $\tau_{a}(\chi)=\tau_{b}(\chi)$.

Proposition 1.2. If $b$ is an integer of $O_{K}$ prime to the ideal $I$, then

$$
G(\chi, a b)=\bar{\chi}(b) G(\chi, a) .
$$

In particular, if $K=\mathbb{Q}, I=n \mathbb{Z}$ with $n$ a natural number and $a=k / n$ with $(k, n)=1$, then

$$
\tau_{a}(\chi)=\bar{\chi}(k) \tau(\chi) .
$$


Proof. Let $b$ be defined as before. Since $b$ is prime to $I$ then $b$ is invertible modulo $I$ and multiplication by $b$ on the set of residues modulo $I$ is an isomorphism between the two sets both of which are sets of residues modulo $I$. Furthermore the Gauss sums does not depend on the set of residues modulo $I$, so that:

$$
\begin{aligned}
G(\chi, a b) & =\sum_{x \bmod I} \chi(x) \exp \left(2 \pi i \operatorname{Tr}_{K / \mathbb{Q}}(a b x)\right) \\
& =\sum_{y \bmod I} \chi\left(y b^{-1}\right) \exp \left(2 \pi i \operatorname{Tr}_{K / \mathbb{Q}}(a y)\right) \\
& =\chi\left(b^{-1}\right) \sum_{y \bmod I} \chi(y) \exp \left(2 \pi i \operatorname{Tr}_{K / \mathbb{Q}}(a y)\right) \quad y=b^{-1} \\
& =\overline{\chi(b)} G(\chi, a) .
\end{aligned}
$$

Remark From this proposition we know that the Gauss sums for a fixed character $\chi$ of $G[I]$ are fully determined by $G(\chi, 1)$.

Proposition 1.3. Let $I=I_{1} \cdots I_{r}$ be a factorization of $I$ into factors relatively prime in pairs and let $\chi=\chi_{1} \cdots \chi_{r}$ be the factorization of the character $\chi$ of $G[I]$. If a lies in $I^{-1} \mathfrak{D}(K / \mathbb{Q})^{-1}$ and for $i=1,2, \cdots, r$ the elements $a_{i}$ belong to $I_{i}^{-1} \mathfrak{D}(K / \mathbb{Q})^{-1}$ and satisfy $a-a_{i} \in J_{i}^{-1} \mathfrak{D}(K / \mathbb{Q})^{-1}$ with $J_{i}=I / I_{i}$ then

$$
G(\chi, a)=\prod_{i=1}^{r} G\left(\chi_{i}, a_{i}\right) .
$$

Proof. Let $x \in O_{K}$, relatively prime to $I$. Choose $x_{i} \in O_{K}$ with $x_{i} \equiv x \bmod I_{i}$. Choose $x_{i} \equiv 1 \bmod I_{j}$ for $i \neq j$. This is a consequence of the Chinese remainder theorem whose proof is given in the appendix. Then we will have for elements $y_{1}, \cdots, y_{r} \in O_{K}$ such that, $y_{i} \equiv 1 \bmod I_{i}$ and $y_{i} \in I_{j}$ for $j \neq i$,

$$
x_{1} y_{1}+\cdots+x_{r} y_{r} \equiv x \bmod I
$$

and thus

$$
\begin{gathered}
G(\chi, a)=\sum_{\substack{x \in G\left[I_{i}\right] \\
i=1, \cdots, r}} \chi_{1}\left(x_{1}\right) \cdots \chi_{r}\left(x_{r}\right) \exp \left(2 \pi i \operatorname{Tr}_{K / \mathbb{Q}}(a x)\right) \\
\left.=\prod_{i=1}^{r} \sum_{x \in G\left[I_{i}\right]} \chi_{1}\left(x_{1}\right) \cdots \chi_{r}\left(x_{r}\right) \exp \left(2 \pi \operatorname{Tr}\left(a x_{i} y_{i}\right)\right)\right) \\
=\prod_{i=1}^{r} \sum_{x \in G\left[I_{i}\right]} \chi_{1}\left(x_{1}\right) \cdots \chi_{r}\left(x_{r}\right) \exp \left(2 \pi \operatorname{Tr}\left(a_{i} x_{i} y_{i}\right)\right)
\end{gathered}
$$

since $a x_{i} y_{i}-a_{i} x_{i} y_{i} \in D_{K / \mathbb{Q}}^{-1}$ and consequently, the latest equality is equal

$$
\prod_{i=1}^{r} G\left(\chi_{i}, a_{i}\right)
$$


because $\chi_{i}\left(y_{i}\right)=1$.

Corollary 1.4. Let $n=n_{1} \cdots n_{r}$ be the factorization of a natural number $n$ into mutually prime factors, let $\chi$ be a character of $G[n \mathbb{Z}]$ (which group we shall for convenience denote it again by $G[n]$ ) and let $\chi=\chi_{1} \cdots \chi_{r}$ be its factorization into characters of $G[N]$. Then we have the equality

$$
\tau(\chi)=\prod_{i=1}^{r} \chi_{i}\left(n / n_{i}\right) \prod_{i=1}^{r} \tau\left(\chi_{i}\right) .
$$

Proof. Take $a=1 / n$ and apply the last 3 propositions.

Corollary 1.5. If $\operatorname{gcd}(a, I) \neq(1)$ and $\chi$ is a primitive character modulo $I$, then

$$
G(\chi, a)=0
$$

ProOF. Let

$$
\operatorname{gcd}(a, I)=I_{1} \text { and } \alpha \equiv 1 \bmod \frac{I}{I_{1}}, \text { with } \operatorname{gcd}(\alpha, f)=(1),
$$

then

$$
a \alpha \equiv a \bmod I .
$$

Thus

$$
G(\chi, a \alpha)=\bar{\chi}(\alpha) G(\chi, a)=G(\chi, a)
$$

therefore it follows that

$$
(1-\chi(\alpha)) G(\chi, a)=0
$$

We have to show that at least one $\alpha$ satisfies $\chi(\alpha) \neq 1$. Now suppose that the conditions on $\alpha$ extend to all residue classes modulo $f$, then at least one of them satisfies $\chi(\alpha) \neq 1$ otherwise $\chi$ is a character modulo $\frac{I}{I_{1}}$ which contradicts the hypothesis that $\chi$ is primitive modulo I. For this value of $\alpha$ we have $(1-\chi(\alpha)) G(\chi, a)=0$ implies $G(\chi, a)=0$.

We define here the local Gauss sum.

Let $F$ be a finite extension of the $p$-adic field $\mathbb{Q}_{p}$ and $\mathfrak{P}$ the prime ideal above $(p)$. If we denote $U=U_{0}=O_{F}^{\times}$the group of local units of $F$ and $U_{n}=1+\mathfrak{P}^{n} n>0$ the $n^{\text {th }}$ unit group of $F$, then for any multiplicative character $\chi$ of $F, \operatorname{ker}(\chi)$ contains at least a $U_{m}$ for some integer $m \geq 0$. Since the sequence of the $\left(U_{n}\right)$ is decreasing, $m$ is chosen smallest such that $U_{m} \subset \operatorname{ker}(\chi)$ then $f_{\chi}=\mathfrak{P}^{m}$ is called conductor of $\chi$. If $m=0$ we say that $\chi$ is unramified, otherwise it is ramified. In both cases we have different consequences for the definition of $\chi$. If $\chi$ is ramified, then $\chi(x)=0$ for all $x \in F$ however for $\chi$ unramified, we consider $\chi$ as a function on the group of fractional ideals $\mathfrak{I}=F^{\times} / U$.

If $\lambda$ is any additive character of $F$ which is trivial on $\mathbb{Z}_{p}$ then, we define the local Gauss sum as follow:

Definition 3.1. The local Gauss sums is

$$
\tau(\chi, a)=\sum_{\substack{x \in U \\ x \bmod 1+f_{\chi}}} \chi(x) \exp \left(2 \pi i \lambda\left(\operatorname{Tr}_{F / \mathbb{Q}_{p}}\left(a^{-1} x\right)\right)\right)
$$

where $a \in F^{\times}$is such that $(a)=f_{\chi} \mathfrak{D}_{F}$ and $\mathfrak{D}_{F}$ is the local different of $F / \mathbb{Q}_{p}$. 


\section{Remark}

- $\tau(\chi, a)$ does not depend on the choice of the residue $\bmod \left(1+f_{\chi}\right)$.

- If $\chi$ is unramified, then the residue class modulo $1+f_{\chi}$ is restricted to a single element, therefore $\tau(\chi, a)=\chi\left(\mathfrak{D}_{F}\right)$. This means that $\tau(\chi, a)$ is a root of unity, since $\chi$ is of finite order.

One of the most important properties for these definitions of Gauss sums is the following.

Proposition 1.6. Let $\mathfrak{P}$ be a non zero prime ideal of $O_{K}$ and $\chi$ a primitive character of $G\left[\mathfrak{P}^{N}\right]$. Let $\mathfrak{P}=\mathfrak{P}_{1}, \mathfrak{P}_{2}, \cdots, \mathfrak{P}_{g}$ be all the prime ideals of $O_{K}$ lying above the ideal $p \mathbb{Z}=\mathfrak{P} \cap \mathbb{Z}$, denote by $\mathfrak{P}^{m}$ the maximal power of $\mathfrak{P}$ dividing the different $\mathfrak{D}(K / \mathbb{Q})$. We choose an element $\Pi$ of $K$ such that $\Pi O_{K}=\mathfrak{P}_{1} I^{-1}, I \subset O_{K}, \mathfrak{P}_{1} \backslash X$. If we denote the character of $U\left(K_{\mathfrak{P}}\right) / U_{N}\left(K_{\mathfrak{P}}\right)$ induced by $\chi$ by the same letter and put $a=\Pi^{-m-N}$, the Gauss sum $G(\chi, a)$ coincides with the Gauss sum $\tau(\chi, a)$.

PROOF. This proof is essentially based on the relation from local to global argument. In fact the global trace is the sum of local traces, such that

$$
\operatorname{Tr}_{K / \mathbb{Q}}(x)=\sum_{i=1}^{g} T_{K_{\mathfrak{P}_{i}} / \mathbb{Q}_{p}}(x) .
$$

Moreover, for $x \in K$ with $\left(x O_{K}, \mathfrak{P}_{2} \cdots \mathfrak{P}_{g}\right)=(1)$ the quotient $x / \Pi^{m+N}$ is an integral element in $K_{\mathfrak{P}_{i}}$ for $i=2, \cdots, g$; thus

$$
\exp \left(2 \pi\left(i \lambda\left(\operatorname{Tr}_{K / \mathbb{Q}}\left(\Pi^{-m-N} x\right)\right)\right)\right)=1
$$

for $i=2, \cdots, g$. In fact $\operatorname{Tr}_{K / \mathbb{Q}}\left(\Pi^{-m-N} x\right)$ is a rational number whose denominator is a power of $p$, whence $\lambda\left(\operatorname{Tr}_{K / \mathbb{Q}}\left(\Pi^{-m-N} x\right)\right)$ coincides with the fractional part of $\operatorname{Tr}_{K / \mathbb{Q}}\left(\Pi^{-m-N} x\right)$ and this leads to

$$
\exp \left(\operatorname{Tr}_{K / \mathbb{Q}}\left(2 \pi i \Pi^{-m-N} x\right)\right)=\exp \left(2 \pi i \lambda\left(\operatorname{Tr}_{K_{\mathfrak{P}_{1}} / \mathbb{Q}_{p}}\left(\Pi^{-m-N} x\right)\right)\right) .
$$

We then apply once more the Chinese Remainder Theorem to choose a suitable set of representatives $x_{1}, \cdots x_{k}$ of $G\left[\mathfrak{P}^{N}\right]$ that satisfies $\left(x O_{K}, \mathfrak{P}_{2} \cdots \mathfrak{P}_{g}\right)=(1)$. This representative system of $G\left[\mathfrak{P}^{N}\right]$ is also a representative system of $U\left(K_{\mathfrak{P}_{1}}\right) / U_{N}\left(K_{\mathfrak{P}_{1}}\right)$. Thus for $a=\Pi^{-m-N}$, we deduce from the equality above that

$$
G(\chi, a)=\sum_{i=1}^{k} \chi\left(x_{i}\right) \exp \left(2 \pi i \lambda\left(\operatorname{Tr}_{K_{\mathfrak{P}_{1}} / \mathbb{Q}_{p}}\left(\Pi^{-m-N} x\right)\right)\right)=\tau(\chi, a) .
$$

Corollary 1.7. Let $\chi$ be a primitive character of $G\left[\mathfrak{P}^{n}\right]$, let $\mathfrak{P}^{m}$ be the maximal power of $\mathfrak{P}$ which divides $\mathfrak{D}(K / \mathbb{Q})$ and finally let a be any element of $\mathfrak{P}^{-m-N}$ $\mathfrak{P}^{-m-N+1}$. Then the Gauss sum $G(\chi, a)$ differs from $\tau(\chi, 1)$ only by a factor which is a root of unity.

Proof. Let $\Pi$ be a uniformizer of $\mathfrak{P}$, then if $b$ is an element of $K_{\mathfrak{P}}$ such that $b$ and $b^{-1}$ are integral in $K_{\mathfrak{P}}$, then for every $c$ in $\mathfrak{P}^{-m-N}$ the sums $G(\chi, c)$ and $G(\chi, b c)$ differ by a factor which is a root of unity. Thus choose $b$ to be $a / \Pi^{-m-N}$ and $c=\Pi^{-m-N}$. 
Corollary 1.8. Let $I=\prod_{i=1}^{r} \mathfrak{P}_{i}^{N_{i}}$ and for any $i=1, \cdots, r$ let $\mathfrak{P}_{i}^{m_{i}}$ be the maximal power of $\mathfrak{P}_{i}$ which divides the different $D_{K / \mathbb{Q}}$. Moreover, let $n_{i}\left(\mathfrak{P}_{\mathfrak{i}}\right)$ be the exponent corresponding to the prime ideal $\mathfrak{P}_{i}$. If a is an element of $I^{-1} D_{K / \mathbb{Q}}^{-1}$, which satisfies $n_{i}(a)=-m_{i}-N_{i}$ for $i=1,2, \cdots, r$ and $\chi$ is a primitive character of $G[I]$, then

$$
|G(\chi, a)|=N(I)^{1 / 2} \text {. }
$$

Proof. We have proved that $G(\chi, a)$ can split as a product of $G\left(\chi_{i}, a_{i}\right)$ for suitable primitive characters $\chi_{i}$ defined on $G\left[\mathfrak{P}_{\mathfrak{i}}{ }^{N_{i}}\right]$, and integers $a_{i}$. To prove our theorem, you need to do it only locally for $I=\mathfrak{P}_{i}^{N}$.

- If $\chi$ is unramified, then the conductor of $\chi$ is $I=(1)$ and therefore

$$
G(\chi, a) \overline{G(\chi, a)}=\chi\left(\mathfrak{D}_{F}\right) \overline{\chi\left(\mathfrak{D}_{F}\right)}=1 .
$$

- If $\chi$ is ramified, then the conductor of $\chi$ is $I=\mathfrak{P}^{n}$.

$$
\begin{aligned}
G(\chi, a) \overline{G(\chi, a)} & =\sum_{x, y \in O^{\times} / U_{n}} \chi(x) \exp \left(2 \pi i \lambda\left(\operatorname{Tr}_{F / \mathbb{Q}_{p}}\left(a^{-1} x\right)\right)\right) \overline{\chi(y) \exp \left(2 \pi i \lambda\left(\operatorname{Tr}_{F / \mathbb{Q}_{p}}\left(a^{-1} y\right)\right)\right)} \\
& =\sum_{x, y \in O^{\times} / U_{n}} \chi(x y) \chi^{-1}\left(y a^{-1}\right) \exp \left(2 \pi i \lambda\left(\operatorname{Tr}_{F / \mathbb{Q}_{p}}\left(a^{-1}(x y-y)\right)\right)\right) \\
& =\sum_{x, y \in O^{\times} / U_{n}} \chi(x) \exp \left(2 \pi i \lambda\left(\operatorname{Tr}_{F / \mathbb{Q}_{p}}\left(a^{-1} y(x-1)\right)\right)\right)
\end{aligned}
$$

furthermore

$$
\begin{aligned}
\sum_{y \in O^{*} / U_{n}} \exp \left(2 \pi i \lambda\left(\operatorname{Tr}_{F / \mathbb{Q}_{p}}\left(a^{-1} y(x-1)\right)\right)\right) & =\sum_{y \in O / \mathfrak{P}^{n}} \exp \left(2 \pi i \lambda\left(\operatorname{Tr}_{F / \mathbb{Q}_{p}}\left(a^{-1} y(x-1)\right)\right)\right) \\
& -\sum_{y \in \mathfrak{P} / \mathfrak{P}^{n}} \exp \left(2 \pi i \lambda\left(\operatorname{Tr}_{F / \mathbb{Q}_{p}}\left(a^{-1} y(x-1)\right)\right)\right) .
\end{aligned}
$$

On the one hand,

$$
\begin{aligned}
\sum_{y \in O / \mathfrak{P}^{n}} \exp \left(2 \pi i \lambda\left(\operatorname{Tr}_{F / \mathbb{Q}_{p}}\left(a^{-1} y(x-1)\right)\right)\right) & =0 \quad \text { if } x \not \equiv 1 \bmod \mathfrak{P}^{n} \\
& =N_{F / \mathbb{Q}_{p}}(\mathfrak{P})^{n}=N_{F / \mathbb{Q}_{p}}(I), \quad \text { otherwise. }
\end{aligned}
$$

on the another hand

$$
\begin{aligned}
\sum_{y \in \mathfrak{P} / \mathfrak{P}^{n}} \exp \left(2 \pi i \lambda\left(\operatorname{Tr}_{F / \mathbb{Q}_{p}}\left(a^{-1} y(x-1)\right)\right)\right) & =\sum_{y \in O / \mathfrak{P}^{n-1}} \exp \left(2 \pi i \lambda\left(\operatorname{Tr}_{F / \mathbb{Q}_{p}}\left(a^{-1} y \Pi(x-1)\right)\right)\right) \\
& =0 \quad \text { if } x \not \equiv 1 \bmod \mathfrak{P}^{n-1} \\
& =N_{F / \mathbb{Q}_{p}}(\mathfrak{P})^{n-1} \quad \text { otherwise. }
\end{aligned}
$$

where $\Pi$ is a uniformizer for $\mathfrak{P}$.

It then follows that

$$
G(\chi, a) \overline{G(\chi, a)}=N_{F / \mathbb{Q}_{p}}\left(\mathfrak{P}^{n}\right)-\sum_{x \in U_{n-1} / U_{n}} \chi(x) N_{F / \mathbb{Q}_{p}}\left(\mathfrak{P}^{n-1}\right) .
$$


Since

$$
\sum_{x \in U_{n-1} / U_{n}} \chi(x)=0, \quad \text { by mean of character relation, }
$$

the assertion then follows.

Corollary 1.9. If $\chi$ is a primitive character of $G[I]$, then

$$
G(\bar{\chi}, a)=\chi(-1) \overline{G(\chi, a)}
$$

Proof. This is just a consequence of the above corollary.

Remark Let $\mathfrak{P}=(p)$ prime ideal in $\mathbb{Z}$ and $I=p^{r}, \chi$ defined as before. We put again

$$
\zeta=\zeta_{p^{r}}=\exp \left(\frac{2 \pi i}{p}\right)
$$

Then

$$
G(\chi, a)=\sum_{x \bmod p^{r}} \chi(x) \zeta^{a x}
$$

We then have the following proposition.

Theorem 3.1. For $a \not \equiv 0 \bmod p G(\chi, a)=0$ if and only if $r>0$ and $\chi$ is not $a$ primitive character modulo $p^{r}$.

PROOF. It is necessary to prove this theorem for $a=1$.

For $r=1, \chi$ must either be primitive or else be a principal character modulo $p$, where in the later case

$$
G(\chi, 1)=\zeta^{1}+\zeta^{2}+\cdots+\zeta^{p-1}=-1 \neq 0 .
$$

For the remainder of the proof, we assume that $r \geq 1$ and that $\chi$ is primitive if $r=1$.

$$
\begin{aligned}
|G(\chi, 1)|^{2} & =\sum_{x \bmod p^{r}} \chi(x)^{-1} \zeta^{-x} \sum_{y \bmod p^{r}} \chi(y) \zeta^{y} \\
& =\sum_{x, y} \chi\left(x^{-1} y\right) \zeta^{y-x} \\
& =\sum_{\substack{x \bmod p^{r} \\
x \neq 0 \bmod p}} \sum_{\bmod p^{r}} \chi(t) \zeta^{t x-x} \text { where } t=x^{-1} y \\
& =\sum_{t} \sum_{x \neq 0 \bmod p} \zeta^{x(t-1)} \\
& =\sum_{t \bmod p^{r}} \chi(t) \sum_{x \bmod p^{r}} \zeta^{x(t-1)}-\sum_{t \bmod p^{r}} \chi(t) \sum_{z \bmod p^{r-1}} \zeta^{p z(t-1)}
\end{aligned}
$$

In the first term if $t \not \equiv 0 \bmod p^{r}$, the sum on $x$ vanishes, therefore the first term sums to $p^{r}$. In the same way, when $r>1$ and $t \not \equiv 0 \bmod p^{r-1}$, the sum on $z$ is zero. 
It then follows that,

$$
|G(\chi, 1)|^{2}=p^{r}-p^{r-1} \sum_{\substack{t \\ t \equiv 1}} \chi(t) .
$$

When $r=1$ and $\chi$ is primitive, the sum is the same as $\sum_{x \bmod p} \chi(t)$ which is zero. When $r>1$ and $\chi$ is primitive, the sum is again zero, because in this case the restriction of $\chi$ on the subgroup $\left\{t \equiv 1 \bmod p^{r-1}\right\}$ of $G\left[P^{r}\right]$, is a non trivial character. Finally if $r>1$ and $\chi$ is not primitive, then $\chi$ is certainly trivial on $\left\{t \equiv 1 \bmod p^{r-1}\right\}$, so that the last sum is $p$.

In the next properties we suppose without loss of generality that the ideal $I$ is a prime ideal $\mathfrak{P}$ and $\chi$ is Dirichlet character $\bmod \mathfrak{P}$ of order $m$. We must then observe that in the Gauss sums, we sum over the set of residues $\bmod \mathfrak{P}$, therefore $G[\mathfrak{P}] \equiv(\mathbb{Z}[\zeta] / \mathfrak{P})^{*}$. Since $\mathbb{Z}[\zeta] / \mathfrak{P}$ is a field with $p^{n}=q=N_{K(\zeta) / \mathbb{Q}}(\mathfrak{P})$ elements, it is isomorphic to $\mathbb{F}_{q}$. We then use this isomorphism to look at functions on $\mathbb{Z}[\zeta] / \mathfrak{P}$ as functions defined on $\mathbb{F}_{q}$, so that we can see characters on $G[\mathfrak{P}]$ as characters on the multiplicative group $\mathbb{F}_{q}^{*}$.

Remark : Since $\operatorname{gcd}(q-1, q)=1$, then $\mathbb{Q}\left(\zeta_{q-1}, \zeta_{q}\right)=\mathbb{Q}\left(\zeta_{q-1}\right) \mathbb{Q}\left(\zeta_{q}\right)$. Therefore the Galois group $\operatorname{Gal}\left(\mathbb{Q}\left(\zeta_{q-1}, \zeta_{q}\right) / \mathbb{Q}\right)$ is equal to the direct product $\operatorname{Gal}\left(\mathbb{Q}\left(\zeta_{q-1}\right) / \mathbb{Q}\right) \times$ $\operatorname{Gal}\left(\mathbb{Q}\left(\zeta_{q}\right) / \mathbb{Q}\right)$. This means that a $\mathbb{Q}$-automorphism of $\left.\mathbb{Q}\left(\zeta_{q-1}, \zeta_{q}\right) / \mathbb{Q}\right)$ is of the form $(\sigma, \tau)$ where $\sigma \in \operatorname{Gal}\left(\mathbb{Q}\left(\zeta_{q-1}\right) / \mathbb{Q}\right)$ leaves invariant $\zeta_{q}$ and $\tau \in \operatorname{Gal}\left(\mathbb{Q}\left(\zeta_{q}\right) / \mathbb{Q}\right)$ leaves invariant $\zeta_{q-1}$. This actually has an effect on the Gauss sums.

Let $\chi$ be a non trivial primitive Dirichlet character modulo $\mathfrak{P}$ of order $m \mid q-1$ and $a \in K$ is such that $a \Pi \in O_{K}$, where $\Pi$ generates $\mathfrak{P}$ in $K=\mathbb{Z}\left[\zeta_{m}\right]$.

Proposition 1.10. 1 -The Gauss sum $G(\chi, a)$ is an algebraic integer in $\mathbb{Q}\left(\zeta_{m}, \zeta_{q}\right)$.

$2-\sigma \cdot G(\chi, a)=G\left(\chi^{c}, a\right)$ and $\tau \cdot G(\chi, a)=G(\chi, a b)$ where $\sigma$ is the automorphism of $\mathbb{Q}\left(\zeta_{m}\right) / \mathbb{Q}$ that maps $\zeta_{m} \rightarrow \zeta_{m}^{c}$ for $c \in G[m]$ and $\tau$ is the isomorphism of $\mathbb{Q}\left(\zeta_{q}\right) / \mathbb{Q}$ that maps $\zeta_{q} \rightarrow \zeta_{q}^{b}$ for some $b \in \mathbb{F}_{p}^{*}$.

$3-G(\chi, a)^{m}$ is an algebraic integer in $\mathbb{Q}\left(\zeta_{m}\right)$ independent of $a \neq 0$.

$4-G(\chi, a)^{m}$ and $G\left(\chi^{\prime}, a\right)^{m}$ are conjugate in $\mathbb{Q}\left(\zeta_{m}\right)$ if $\chi$ and $\chi^{\prime}$ are characters of the same order dividing $m$.

Proof. 1 ) It is a simple observation that the Gauss sum is a sum of the product of $\zeta_{m}$ and $\zeta_{q}$ and both are algebraic integers in $\mathbb{Q}\left(\zeta_{m}, \zeta_{q}\right)$.

2 ) It is a simple fact. $\chi(x)$ is an $m^{\text {th }}$ root of unity which is left invariant under the $\tau$ and $\exp \left(2 \pi i \operatorname{Tr}_{K / \mathbb{Q}}(x)\right)$ is a $q^{\text {th }}$ root of unity which is invariant under $\sigma$. Since $\chi^{\sigma}(x)=\sigma(\chi(x))=(\chi(x))^{c}$ are non-trivial and since $\tau . G(\chi, a)=G(\chi, b a)$ the assertion follows

3 ) From 2) we see that

$$
\tau \cdot(G(\chi, a))^{m}=(\tau G(\chi, a))^{m}=\left(\chi(b)^{-1} G(\chi, a)\right)^{m}=G(\chi, 1)^{m},
$$

since $\chi(b)$ is an $m^{\text {th }}$ root of unity. Therfore $G(\chi, a)$ is invariant under $\operatorname{Gal}\left(\mathbb{Q}\left(\zeta_{p} /\right) \mathbb{Q}\right)$ 
4 ) this a consequence of 2) and 3).

Remark From the following propositions, we see that, for primitive characters modulo an ideal $I$ of order $m$, we know the absolute norm of the Gauss sum, and that its $m^{\text {th }}$ power is an algebraic integer in the field $\mathbb{Q}\left(\zeta_{m}\right) / \mathbb{Q}$. Since we know the decomposition law in this cyclotomic field, we would attempt to describe the decomposition of this Gauss sum in this field. According to the multipcative properties, we will do this only for ideals I prime. While looking for the factorization of the $\mathrm{m}^{\text {th }}$ power of the Gauss sum, we do not need to consider the action of $\tau \in G a l\left(\mathbb{Q}\left(\zeta_{q}\right) / \mathbb{Q}\right)$, therefore one can describe this when $q$ is a prime.

\section{Factorization of Gauss sums}

We have already noticed that for Dirichlet character $\chi$ of prime conductor the integral ideal generated by the $m^{\text {th }}$ power of Gauss sum lies in the subring of $\mathbb{Z}\left[\zeta_{p(q-1)}\right]$, precisely in the ring $O_{K}=\mathbb{Z}\left[\zeta_{m}\right]$. Its norm is a power of a prime $p$ and therefore factors of $p$ in $O_{K}$ appear in its decomposition. One should ask the question which of the factors of $p$ and for each of the factors, with what power does it occur. This involves the $\Pi$-adic analysis of the Gauss sums which was completely explained by Stickelberger [S.L], pp. 6-13.

Theorem 3.2. Let $\mathfrak{p} \mid p$ be a prime ideal in $O_{K}$ and $\chi$ the corresponding Dirichlet character of order $m$ prime to $p$. Then

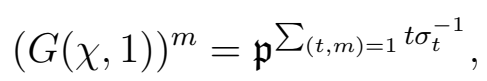

where $\sigma_{t}$ maps $\zeta=\zeta_{m}$ to $\zeta^{t}$ with $\operatorname{gcd}(t, m)=1$.

We set without loss of generality that $\zeta_{m}=\exp (2 \pi i / m)$

Let $\mathfrak{P} \mid \mathfrak{p}$ be a prime divisor of the ideal $(p)$ in the ring of integers $\mathbb{Z}\left[\zeta_{p(q-1)}\right]$, and consider the residue class field $\kappa=\left\{0,1, g, \cdots, g^{q-2}\right\}$ where $g=\zeta_{q-1}+\mathfrak{P}$. Let $a \in\{1,2, \cdots, q-2\}$. A theorem of Stickelberger, see [S.L] or $[\mathbf{I} . \mathbf{R}]$ chapter 11, states that the highest power of $\mathfrak{P}$ dividing the ideal generated by the Gauss sum

$$
\sum_{i=0}^{q-2} \zeta_{q-1}^{-a i} \zeta_{p}^{T r}\left(g^{i}\right)
$$

is equal to the digit sum $s_{p}(a)$ in the $p$-adic expansion of $a$. Since $\mathbb{F}=F_{q}$ and $\kappa$ are isomorphic fields, there exists a primitive element $\gamma$ in $\mathbb{F}$ which is mapped onto $g$ under the isomorphism. Now the character $\chi$, defined by $\chi(\gamma)=\zeta_{q-1}$ is a multiplicative character of order $q-1$ of $\mathbb{F}$. Thus the highest power of $\mathfrak{P}$ dividing $\left(G\left(\bar{\chi}^{a}\right)\right)$ is equal to $s_{p}(a)$.

Let $a=(q-1) / N$ for some divisor $N$ of $q-1$. Since $G\left(\bar{\chi}^{a}\right) \in \mathbb{Q}\left(\zeta_{N}\right)$ and $G\left(\bar{\chi}^{a}\right) \overline{G\left(\bar{\chi}^{a}\right)}=p^{m}$, the only possible divisors of the ideal $(G(\chi))$ in $\mathbb{Z}\left[\zeta_{N}\right]$ are the prime divisors of $(p)$. Let $(p)=\prod_{i=1}^{t} P_{i}$ and $(p)=\prod_{i=1}^{t_{1}} \mathfrak{P}_{i}^{p-1}$, with $t=\varphi(N) / \operatorname{ord}_{N}(p)$ and $t_{1}=\varphi(q-1) / m$, be the prime ideal decomposition of $(p)$ in $\mathbb{Z}\left[\zeta_{N}\right]$ and in $\mathbb{Z}\left[\zeta_{p(q-1)}\right]$, respectively. This refers to the factorization of primes in cyclotomic fields. It then follows that ord $_{\mathfrak{P}_{i}}\left(P_{i} \mathbb{Z}\left[\zeta_{p(q-1)}\right]\right)=p-1$, for $i=1, \cdots, t$. By lifting the prime ideal decomposition of the ideal $G\left(\bar{\chi}^{a}\right) \mathbb{Z}\left[\zeta_{N}\right]$ into $\mathbb{Z}\left[\zeta_{p(q-1)}\right]$, we obtain 
$(p-1) \operatorname{ord}_{P}\left(G\left(\bar{\chi}^{a}\right)\right)=\operatorname{ord}_{\mathfrak{P}} G\left(\bar{\chi}^{a}\right)$ for some $P \in\left\{P_{1}, \cdots, P_{g}\right\}$, since $P_{i} \mathbb{Z}\left[\zeta_{p(q-1)}\right]$ and $P_{j} \mathbb{Z}\left[\zeta_{p(q-1)}\right]$ are relatively prime for $i \neq j$. Therefore, ord $_{P}\left(G\left(\bar{\chi}^{a}\right)\right)=s_{p}(a) /(p-1)$. Let $P^{\prime}$ be another prime divisor of $\left(G\left(\bar{\chi}^{a}\right)\right)$ in $\mathbb{Z}\left[\zeta_{N}\right]$. We know that $P^{\prime}=\sigma_{i}^{-1}(P)$ for $\sigma_{i} \in \operatorname{Gal}(K / \mathbb{Q})$ (see $[\mathbf{I} . \mathbf{R}]$ chap.12). Furthermore, $\sigma_{1}(P)=\sigma_{2}(P)$ if and only if $\sigma_{2}^{-1} \sigma_{1} \in G_{p}=\{\sigma \in \operatorname{Gal}(K / \mathbb{Q}) \mid \sigma(P)=P\} \subset \operatorname{Gal}(\mathbb{F} / \mathbb{Q})$. Thus $P^{\prime}=\sigma(P)$ if and only if $\sigma \in \sigma_{i}^{-1} G_{p}$.

Let $S \subset G[N]$ be a complete set of representatives of the cosets of the principal subgroup $<p>$ in $G[N]$. Since $G[N] \cong \operatorname{Gal}(\mathbb{F} / \mathbb{Q})$ by $i \mapsto \sigma_{i}: \zeta_{N} \mapsto \zeta_{N}^{i}$, then $G_{p}=<\sigma_{p}>([\mathbf{I} . \mathbf{R}]$ chap. 13) and we then have

$$
\left(G\left(\bar{\chi}^{a}\right)\right)=\prod_{i \in S} \sigma_{i}(P)^{b_{i}}
$$

where $b_{i}=\operatorname{ord}_{\sigma_{i}^{-1}(P)}\left(G\left(\bar{\chi}^{a}\right)\right)$. We see without any difficulty that $b_{i}=\operatorname{ord}_{P}\left(\sigma_{i}\left(G\left(\bar{\chi}^{a}\right)\right)\right)$, and it is easy to see that $\operatorname{ord}_{\mathfrak{P}} \sigma_{i}\left(G\left(\bar{\chi}^{a}\right)\right)=s_{p}($ ai $)$ ([I.R] chap. 14). Now by similar argument as described above, we get $b_{i}=s_{p}(a i) /(p-1)$. Thus the highest power of $p$ dividing $G\left(\bar{\chi}^{\left.\frac{q-1}{N} a\right)}\right.$ is

$$
h:=\frac{1}{p-1} \min \left\{s_{p}\left(\frac{q-1}{N} a\right) \mid \quad a \in S\right\} .
$$

We may replace $S$ by $G[N]$ since $s_{p}(j)=s_{p}(p j)$ for all integer $j>0$. It also follows that the highest power of a factor of $p$ dividing $G\left(\chi^{\frac{q-1}{N} a}\right)=G\left(\bar{\chi}^{\frac{q-1}{N}(N-a)}\right)$ is equal to $h$, since $\operatorname{gcd}(N-a, N)=1$ if and only if $\operatorname{gcd}(a, N)=1$.

We give a table of the factorization of the Gauss sums for some prime cases. Here the ideals $\mathfrak{p}_{i}$ are factors of $(p)$ in $\mathbb{Z}\left[\zeta_{m}\right]$. 
TABle 1

\begin{tabular}{|c|c|c|}
\hline$m$ & $p \equiv a \bmod m$ & Factorization of $m^{\text {th }} \quad$ power Gauss sums \\
\hline 2 & 1 & $\mathfrak{p}_{1}=(p)$ \\
\hline 3 & 1 & $\mathfrak{p}_{1}^{2} \mathfrak{p}_{2}$ \\
\hline 3 & 2 & $\mathfrak{p}_{1}^{2} \mathfrak{p}_{2}=(p)^{3}$ \\
\hline 4 & 1 & $\mathfrak{p}_{1}^{3} \mathfrak{p}_{2}$ \\
\hline 4 & 3 & $\mathfrak{p}_{1}^{3} \mathfrak{p}_{2}=(p)^{4}$ \\
\hline 5 & 1 & $\mathfrak{p}_{1}^{4} \mathfrak{p}_{2}^{2} \mathfrak{p}_{3}^{3} \mathfrak{p}_{4}$ \\
\hline 5 & 2 & $\mathfrak{p}_{1}^{4} \mathfrak{p}_{2}^{2} \mathfrak{p}_{3}^{3} \mathfrak{p}_{4}=\mathfrak{p}_{1}^{5} \mathfrak{p}_{2}^{5}=\left(\mathfrak{p}_{1} \mathfrak{p}_{2}\right)^{5}=(p)^{10}$ \\
\hline 5 & 3 & $\mathfrak{p}_{1}^{4} \mathfrak{p}_{2}^{2} \mathfrak{p}_{3}^{3} \mathfrak{p}_{4}=\mathfrak{p}_{1}^{5} \mathfrak{p}_{2}^{5}=\left(\mathfrak{p}_{1} \mathfrak{p}_{2}\right)^{5}=(p)^{10}$ \\
\hline 5 & 4 & $\mathfrak{p}_{1}^{4} \mathfrak{p}_{2}^{2} \mathfrak{p}_{3}^{3} \mathfrak{p}_{4}=\mathfrak{p}_{1}^{5} \mathfrak{p}_{2}^{5}=\left(\mathfrak{p}_{1} \mathfrak{p}_{2}\right)^{5}=(p)^{5}$ \\
\hline 7 & 1 & $\mathfrak{p}_{1}^{6} \mathfrak{p}_{2}^{3} \mathfrak{p}_{3}^{2} \mathfrak{p}_{4}^{5} \mathfrak{p}_{5}^{4} \mathfrak{p}_{6}$ \\
\hline 7 & 2 & $\mathfrak{p}_{1}^{6} \mathfrak{p}_{2}^{3} \mathfrak{p}_{3}^{2} \mathfrak{p}_{4}^{5} \mathfrak{p}_{5}^{4} \mathfrak{p}_{6}=\mathfrak{p}_{1}^{14} \mathfrak{p}_{6}^{7}=(p)^{7} \mathfrak{p}_{1}^{7}$ \\
\hline 7 & 3 & $\mathfrak{p}_{1}^{6} \mathfrak{p}_{2}^{3} \mathfrak{p}_{3}^{2} \mathfrak{p}_{4}^{5} \mathfrak{p}_{5}^{4} \mathfrak{p}_{6}=\mathfrak{p}_{1}^{21}=(p)^{21}$ \\
\hline 7 & 4 & $\mathfrak{p}_{1}^{6} \mathfrak{p}_{2}^{3} \mathfrak{p}_{3}^{2} \mathfrak{p}_{4}^{5} \mathfrak{p}_{5}^{4} \mathfrak{p}_{6}=\mathfrak{p}_{1}^{14} \mathfrak{p}_{6}^{7}=(p)^{7} \mathfrak{p}_{1}^{7}$ \\
\hline 7 & 5 & $\mathfrak{p}_{1}^{6} \mathfrak{p}_{2}^{3} \mathfrak{p}_{3}^{2} \mathfrak{p}_{4}^{5} \mathfrak{p}_{5}^{4} \mathfrak{p}_{6}=\mathfrak{p}_{1}^{21}=(p)^{21}$ \\
\hline 7 & 6 & $\mathfrak{p}_{1}^{6} \mathfrak{p}_{2}^{3} \mathfrak{p}_{3}^{2} \mathfrak{p}_{4}^{5} \mathfrak{p}_{5}^{4} \mathfrak{p}_{6}=\mathfrak{p}_{1}^{7} \mathfrak{p}_{2}^{7} \mathfrak{p}_{3}^{7}=\left(\mathfrak{p}_{1} \mathfrak{p}_{2} \mathfrak{p}_{3}\right)^{7}=(p)^{7}$ \\
\hline
\end{tabular}


Remark From this table, we really see that although the formula of the decomposition looks complicated, it does have a simple shape. This is due to the use of the Galois theory. For more about the factorization of the Gauss sums, see [w1],[w3]. If $\mathfrak{p} \mid p$ is a prime factor and $\chi$ the corresponding character, then the prime power decomposition is of the form

$$
(G(\chi, a))^{m}=\mathfrak{p}^{\theta^{\prime}}
$$

where $\theta^{\prime}$ is an element of the group ring $\mathbb{Z}[G a l(k / \mathbb{Q})]$. While still working at prime component $\mathfrak{p}$ prime to $m$, we extend the result globally by multiplication. Let $\pi$ be an algebraic integer such that $(\pi)=\mathfrak{p}$. since the ideal generated by $G(\chi, a)^{m}$ is of the form $\mathfrak{p}^{\theta}$, there exists a unit $\varepsilon(\pi, a)$ such that,

$$
G(\chi, a)^{m}=\varepsilon(\pi, a) \pi^{\theta} .
$$

Let's fix any embedding, we have

$$
\left|G(\chi, a)^{m}\right|^{2}=|\varepsilon(\pi, a)|^{2} N_{K / \mathbb{Q}}(\pi)^{m}=N_{K / \mathbb{Q}}(\pi)^{m}
$$

and that implies $|\varepsilon(\pi, a)|=1$ at all places of $K$ in other words, it is root of unity by a theorem of Kronecker.

\section{Gauss sums over finite fields and the Davenport-Hasse relations}

One of the most beautiful results on Gauss sums is the Davenport-Hasse relation. Indeed let $m, p, \mathfrak{p}$ and $q$ be as in the preceding sections. Let $\mu_{n}$ the group of $m^{\text {th }}$ roots of unity in $K$. Then the residue class field modulo $\mathfrak{p}, O_{K} / \mathfrak{p}$, is isomorphic to the finite field $\mathbb{F}_{q}$. Characters on the residue class field modulo $\mathfrak{p}$ are understood to be defined via this isomorphism. The character

$$
\chi_{\mathfrak{p}} \quad: \mathbb{F}_{q}^{\times} \rightarrow \mu_{m}
$$

defined by the relation $\chi_{\mathfrak{p}}(x) \equiv x^{\frac{q-1}{m}} \bmod \mathfrak{p}$ is multiplicative. Any additive character $\lambda_{\alpha}(x)$ define on the residue classes modulo $\mathfrak{p}$ has the form

$$
\exp \left(2 \pi i \operatorname{Tr}_{K / \mathbb{Q}}\left(\frac{\alpha x}{\mathfrak{p}}\right)\right)
$$

for some integer $\alpha$ modulo $\mathfrak{p}$. To simplify the computation we will consider

$$
\psi_{\mathfrak{p}}: \mathbb{F}_{q} \rightarrow \mu_{p}
$$

be the additive character defined by

$$
\psi_{\mathfrak{p}}(x)=\exp \left(2 \pi i T r_{\mathbb{F} q / \mathbb{F}_{p}}(x) / p\right) .
$$

We define the Gauss sums

$\mathfrak{g}_{a}(\mathfrak{p})=\mathfrak{g}\left(a, \chi_{\mathfrak{p}}, \psi_{\mathfrak{p}}\right)=\sum_{x \in \mathbb{F}_{q}^{*}} \chi_{\mathfrak{p}}(x)^{a} \psi_{\mathfrak{p}}(x) \quad\left(\in K\left(\mu_{p}\right)\right)$, which is known to be non-zero

We know already that $\mathfrak{g}_{a}(\mathfrak{p})$ depends only on the congruence class of a modulo $m$ and in particular $\mathfrak{g}_{a}(\mathfrak{p})=-1$ if $a \equiv 0 \bmod m$ and otherwise

$$
\mathfrak{g}_{a}(\mathfrak{p}) \mathfrak{g}_{-a}(\mathfrak{p})=\chi_{\mathfrak{p}}(-1)^{a} q
$$

and

$$
\left|\mathfrak{g}_{a}(\mathfrak{p})\right|=q^{1 / 2} .
$$

The first Hasse-Davenport relation is as follows. 
Theorem 3.3. Let $\ell$ be a divisor of $m$ such that $m=\ell$, then

$$
\prod_{i=0}^{\ell-1} \mathfrak{g}_{a+i d}(\mathfrak{p})=\prod_{\varepsilon: \varepsilon^{\ell=1}} \mathfrak{g}\left(a, \chi_{\mathfrak{p}} \varepsilon, \psi_{\mathfrak{p}}\right)=\chi_{\mathfrak{p}}\left(\ell^{-\ell a}\right) \cdot \mathfrak{g}_{\ell a}(\mathfrak{p}) \prod_{i=1}^{\ell-1} \mathfrak{g}_{i d}(\mathfrak{p}) .
$$

PROOF.

The second Hasse-Davenport relation is as follow. If $\mathbb{E}$ is a finite extension of $\mathbb{F}$, thes shape of the Hasse Davenport formula relates certain Gauss sums over $\mathbb{E}$ to the Gauss sums over $\mathbb{F}$. Indeed if $\mathfrak{P}$ is a prime above $\mathfrak{p}$ in any extension field $K_{1}$ of $K$. Then $O_{K_{1} / \mathbb{Q}} / \mathfrak{P} \cong \mathbb{E}$ as finite field, is such that $E$ is a finite extension of $\mathbb{F}$. We define

$$
\chi_{\mathfrak{P}}^{\prime}=\chi_{\mathfrak{p}} \circ N_{\mathbb{E} / \mathbb{F}_{q}} \text {, and } \psi_{\mathfrak{P}}^{\prime}=\psi_{\mathfrak{p}} \circ t r_{\mathbb{E} / \mathbb{F}_{q}} .
$$

$\chi_{\mathfrak{P}}^{\prime}$ and $\psi_{\mathfrak{P}}^{\prime}$ are respectively multiplicative and additive characters on $\mathbb{E}$.

Theorem 3.4. Let $t_{\mathbb{E} / \mathbb{F}}$ and $N_{\mathbb{E} / \mathbb{F}}$ be the relative trace and relative norm respectively, mappings from $\mathbb{E}$ onto $\mathbb{F}$. Then

$$
\mathfrak{g}_{a}(\mathfrak{P})^{\prime}=(-1)^{m-1} \mathfrak{g}_{a}(\mathfrak{p})^{m},
$$

where $\mathfrak{P} \mid \mathfrak{p}$ in $\mathbb{E} / \mathbb{F}$ of degree $m$ and $\mathfrak{g}_{a}(\mathfrak{P})^{\prime}$ is the corresponding Gauss sum for the characters $\chi_{\mathfrak{P}}^{\prime}, \psi_{\mathfrak{P}}^{\prime}$

PROOF.

\section{The Gauss sum and Jacobi sum as complex numbers}

In this section we give the main relation between Gauss and Jacobi sums.

We introduce a small modification on the definition of the Gauss sum. This version is the computable version where the Gauss sums are embedded in the field of complex number. In fact $\mathbb{Q}\left(\mu_{n}\right) / \mathbb{Q}$ is an extension of degree $\varphi(n) . K=\mathbb{Q}\left(\mu_{n}\right) \otimes_{\mathbb{Q}} \mathbb{R} \cong \mathbb{C}^{\varphi(n) / 2}$ and the ring of integers $\mathbb{Z}\left[\mu_{n}\right]$ is a lattice in $K$.

From here on, we choose the characters to be the power residue symbol $(\dot{\bar{\alpha}})_{n}$ for $\alpha$ and algebraic integer coprime to $n$. Let $\varepsilon$ be any embedding of $\mathbb{Q}\left(\mu_{n}\right)$ into $\mathbb{C}$, then we rewrite the Gauss sums as

$$
\mathfrak{g}(r, \varepsilon, \alpha)=\sum_{x \in G[\alpha]} \varepsilon\left(\left(\frac{x}{\alpha}\right)_{n}\right) \mathbf{e}\left(\frac{r x}{\alpha}\right),
$$

where $\mathbf{e}(x)=\exp \left(2 \pi i \operatorname{Tr}_{K / \mathbb{Q}}(x)\right)$, and $r$ is an algebraic integer in $K$. we will not make any difference between $\operatorname{Tr}_{K / \mathbb{Q}}$ and its unique extension to $\mathbb{C}^{\varphi(n) / 2}$.

All the properties we have described remain unchanged. We will work only at prime algebraic integer argument since multiplicative relations allow one to do so.

Then, $\mathfrak{g}(r, \varepsilon, \pi)$ depends only on the ideal $\mathfrak{p}$ generated by $\pi$. Indeed if we replace $\pi$ by $u \pi$ where $u$ is a unit, then

$$
\mathfrak{g}(r, \varepsilon, u \pi)=\varepsilon\left(\left(\frac{u}{\pi}\right)_{n}\right) \mathfrak{g}(r, \varepsilon, \pi)
$$


implies $(\mathfrak{g}(r, \varepsilon, \pi))^{n}$ does not depend on $\pi$ itself but on the ideal $\mathfrak{p}$ generated by $\pi$. Furthermore if $\pi_{1}$ and $\pi_{2}$ are primes and coprime then for $r$ coprime to $\pi_{1}$ and $\pi_{2}$

$$
\begin{gathered}
\varepsilon\left(\left(\frac{\cdot}{\pi_{1} \pi_{2}}\right)_{n}\right)=\varepsilon\left(\left(\frac{\dot{\epsilon}}{\pi_{1}}\right)_{n}\right) \varepsilon\left(\left(\frac{\dot{ }}{\pi_{2}}\right)_{n}\right) \\
\mathfrak{g}\left(r, \varepsilon, \pi_{1} \pi_{2}\right)=\varepsilon\left(\left(\frac{\pi_{2}}{\pi_{1}}\right)_{n}\right) \varepsilon\left(\left(\frac{\pi_{1}}{\pi_{2}}\right)_{n}\right) \mathfrak{g}\left(r, \varepsilon, \pi_{1}\right) \mathfrak{g}\left(r, \varepsilon, \pi_{2}\right) .
\end{gathered}
$$

Moreover we have the following lemma:

Lemma 3.1. Let $\alpha$ be an algebraic integer prime to $n$. The function $\mathfrak{g}(r, \varepsilon, \alpha)$ vanishes as the Möbiüs function $\mu(\alpha)$ does.

From the multiplicative properties of the Gauss sums, we must suppose that $\alpha$ is a prime $\pi$ and that $r=1$ such that the function $\mathfrak{g}\left(1, \varepsilon, \pi^{r}\right)$ vanishes as the Möbiüs function $\mu\left(\pi^{r}\right)$ does.

PROOF.

$$
\begin{aligned}
\mathfrak{g}\left(1, \varepsilon, \pi^{r}\right) & =\sum_{x \bmod \pi^{r}} \varepsilon\left(\left(\frac{x}{\pi^{r}}\right)_{n}\right) \mathbf{e}\left(\frac{x}{\pi^{r}}\right) \\
& =\sum_{\substack{x \bmod \pi^{r} \\
g c d(\pi x)=1}} \varepsilon^{r}\left(\left(\frac{x}{\pi}\right)_{n}\right) \mathbf{e}\left(\frac{x}{\pi^{r}}\right) \\
& =\sum_{\substack{x_{0} \bmod \pi, x_{1}, \cdots, x_{r-1} \bmod \pi}} \varepsilon^{r}\left(\left(\frac{x_{0}}{\pi}\right)_{n}\right) \mathbf{e}\left(\frac{x_{0}}{\pi^{r}}+\frac{x_{1}}{\pi^{r-1}}+\cdots+\frac{x_{r-1}}{\pi}\right) \\
& =\sum_{x_{0} \bmod \pi} \varepsilon^{r}\left(\left(\frac{x_{0}}{\pi}\right)_{n}\right) \mathbf{e}\left(\frac{x_{0}}{\pi^{r}}\right) \sum_{x_{1}, \cdots, x_{r-1} \bmod \pi} \mathbf{e}\left(\frac{x_{1}}{\pi^{r-1}}+\cdots+\frac{x_{r-1}}{\pi}\right) \\
& =0
\end{aligned}
$$

Another important lemma is giving the relation for $\mathfrak{g}\left(\pi^{\ell}, \varepsilon, \alpha \pi^{r}\right)$. By the same arguments as before we will consider only this case:

$$
\mathfrak{g}\left(\pi^{\ell}, \varepsilon, \alpha \pi^{r}\right)
$$

and the lemma is as follows:

Lemma 3.2. Let $\ell<r$ both integers, the function $\mathfrak{g}\left(\pi^{\ell}, \varepsilon, \pi^{r}\right)$ has the form

$$
\mathfrak{g}\left(\pi^{\ell}, \varepsilon, \pi^{r}\right)= \begin{cases}0 & \text { if } \quad r-\ell>1 \\ -\phi\left(\pi^{r}\right) & r \equiv 0 \bmod n \text { and } r=\ell \\ 0 & r \not \equiv 0 \bmod n \text { and } r=\ell \\ -N\left(\pi^{r-1}\right) & \text { if } r \equiv 0 \bmod n \quad \text { and } r-l=1 \\ N\left(\pi^{r-1}\right) \mathfrak{g}\left(1, \varepsilon^{r}, \pi\right) & \text { if } \quad r \not \equiv 0 \bmod n \quad \text { and } r-l=1\end{cases}
$$

Proof. The crucial point is to write down a simple shape of the elements of the set of residues modulo $\pi^{r}$ which are coprime to $\pi^{r}$. In the following step, we choose this set to be

$$
x_{0}+x_{1} \pi+x_{2} \pi^{2} \cdots+x_{r-1} \pi^{r-1}
$$


where the $x_{i}$ are taken modulo $\pi$ and $x_{0} \not \equiv 0 \bmod \pi, 0 \leq i \leq r-1$.

$$
\begin{aligned}
\mathfrak{g}\left(\pi^{\ell}, \varepsilon, \pi^{r}\right) & =\sum_{x \bmod \pi^{r}} \varepsilon\left(\left(\frac{x}{\pi^{r}}\right)_{n}\right) \mathbf{e}\left(\frac{\pi^{\ell} x}{\pi^{r}}\right) \\
& =\sum_{\substack{x \bmod \pi^{r} \\
g c d(\pi x)=1}} \varepsilon^{r}\left(\left(\frac{x}{\pi}\right)_{n}\right) \mathbf{e}\left(\frac{x}{\pi^{r-\ell}}\right) \\
& =\left\{\begin{array}{lrl}
0 & \text { if } & r-\ell>1 \\
-\phi\left(\pi^{r}\right) & \text { if } & \varepsilon^{r} \equiv 1 \text { and } r=\ell \\
0 & \text { if } & \varepsilon^{r} \neq 1 \text { and } r=\ell \\
-N\left(\pi^{r-1}\right) & \text { if } & \varepsilon^{r} \equiv 1 \text { and } r-l=1 \\
N\left(\pi^{r-1}\right) \mathfrak{g}\left(1, \varepsilon^{r}, \pi\right) & \text { if } & \varepsilon^{r} \neq \equiv \text { and } r-l=1
\end{array}\right.
\end{aligned}
$$

The Galois action of $\operatorname{Gal}(K / \mathbb{Q})$ on $\mathfrak{g}(r, \varepsilon, \pi)$ is as follow: If $\sigma \in \operatorname{Gal}(K / \mathbb{Q})$ is the Galois elements that maps $\zeta_{n}=\zeta$ to $\zeta^{a}$ for some integer $a$, then

$$
\mathfrak{g}(r, \varepsilon, \pi)^{\sigma}=\mathfrak{g}\left(r, \varepsilon^{\sigma}, \pi\right)=\mathfrak{g}\left(r, \varepsilon^{a}, \pi\right)=\mathfrak{g}\left(r, \varepsilon, \pi^{\sigma}\right) .
$$

thus

$$
\mathfrak{g}\left(r, \varepsilon, \pi \pi^{\sigma}\right)=\varepsilon\left(\left(\frac{\pi^{\sigma}}{\pi}\right)_{n}\right) \varepsilon\left(\left(\frac{\pi}{\pi^{\sigma}}\right)_{n}\right) \mathfrak{g}(r, \varepsilon, \pi) \mathfrak{g}\left(r, \varepsilon, \pi^{\sigma}\right) .
$$

We see that, if instead of the prime factor $\pi$ of the prime $p$, we take $p$ itself, then $p$ factors as $p=\prod_{i=1}^{r} \pi_{i}$. It then follows that:

$$
\mathfrak{g}(r, \varepsilon, p)=\prod_{\substack{i=1, j=1 \\ j \neq i}} \varepsilon\left(\left(\frac{\pi_{i}}{\pi_{j}}\right)_{n}\right) \prod_{i=1}^{r} \mathfrak{g}\left(r, \varepsilon, \pi_{i}\right)
$$

Remark This formula looks rather simple but it is a very important one. Since it makes use of the Chinese Remainder Theorem which plays a key role in the the multiplicative relations among the Gauss sums. We also notice that $\mathfrak{g}(r, \varepsilon, p)$ is invariant under all complex embeddings.

Definition 3.2. Let $\chi$ and $\psi$ denote two characters modulo an integral prime $\pi$. The Jacobi sum $J(\chi, \psi)$ is defined by

$$
J(\chi, \psi)=\sum_{x \bmod \pi} \chi(x) \psi(1-x)
$$

Its order is the least common multiple $n$ of the order of $\chi$ and $\psi$, such that the Jacobi sum $J(\chi, \psi) \in \mathbb{Q}\left(\zeta_{n}\right)$.

Note 3.1. The Jacobi sum $J(\chi, \psi)$ defines a symmetric pairing.

The most important properties of the Jacobi sums can be listed as follows.

Theorem 3.5. Let $\chi$ and $\psi$ denote two characters modulo an integral prime $\pi$.

- If $\chi$ and $\psi$ are both trivial, then $J(\chi, \psi)=N_{K / \mathbb{Q}}(\pi)$

- If exactly one of the $\chi^{\prime} s$ or one of the $\psi^{\prime} s$ is trivial, then $J(\chi, \psi)=0$.

- If $\chi$ is nontrivial, then $J(\chi, \bar{\chi})=-\chi(-1)$. 
- If $\chi$ and $\psi$ are such that $\chi, \psi$, and $\chi \psi$ are nontrivial, then

$$
J(\chi, \psi)=\psi(-1) J(\bar{\chi} \bar{\psi}, \psi)=\chi(-1) J(\bar{\chi} \bar{\psi}, \chi)
$$

ProOF. see [B1] pp 57,59

Now as we did for the Gauss sums, we reformulate the Jacobi sums at prime argument for power residue symbol with embedding into the field of complex numbers and extend it as a multiplicative function of $\pi$

$$
J\left((a, b) ; \varepsilon_{1}, \varepsilon_{2}, \pi\right)=\sum_{x \bmod \pi} \varepsilon_{1}\left(\left(\frac{x}{\pi}\right)_{n}\right)^{a} \varepsilon_{2}\left(\left(\frac{1-x}{\pi}\right)_{n}\right)^{b} .
$$

where $a$ and $b$ are taken modulo $n$ and $\varepsilon_{1}, \varepsilon_{2}$ are two fixed complex embeddings of $K=\mathbb{Q}\left(\zeta_{n}\right)$ into $\mathbb{C}$ as before.

We denote $J\left((a, b) ; \varepsilon_{1}, \varepsilon_{2}, \pi\right)$ by $J\left(\varepsilon_{1}, \varepsilon_{2}, \pi\right)$ if $a=b=1$.

The most important relation between Gauss an Jacobi sums is as follows.

Lemma 3.3. Let $\varepsilon_{1}, \varepsilon_{2}$ be two complex characters of $\mu_{n}$. If $\varepsilon_{1} \varepsilon_{2}$ is nontrivial then

$$
\begin{gathered}
\mathfrak{g}\left(r, \varepsilon_{1}, \pi\right) \mathfrak{g}\left(r, \varepsilon_{2}, \pi\right)=J\left(\varepsilon_{1}, \varepsilon_{2}, \pi\right) \mathfrak{g}\left(r, \varepsilon_{1} \varepsilon_{2}, \pi\right) . \\
\left|J\left(\varepsilon_{1}, \varepsilon_{2}, \pi\right)\right|^{2}=N_{K / Q}(\pi)
\end{gathered}
$$

Proof. Indeed,

$$
\mathfrak{g}\left(r, \varepsilon_{1}, \pi\right) \mathfrak{g}\left(r, \varepsilon_{2}, \pi\right)=\sum_{x \bmod } \sum_{y \bmod \pi} \varepsilon_{1}\left(\left(\frac{x}{\pi}\right)_{n}\right) \varepsilon_{2}\left(\left(\frac{y}{\pi}\right)_{n}\right) \mathbf{e}(x+y)
$$

Then put $\gamma=x+y$ and the summation above becomes

$$
\begin{aligned}
& \sum_{\gamma \bmod } \sum_{x} \mathbf{e}(\gamma) \varepsilon_{1}\left(\left(\frac{x}{\pi}\right)_{n}\right) \varepsilon_{2}\left(\left(\frac{\gamma-x}{\pi}\right)_{n}\right) \\
& =\sum_{\substack{x \bmod \pi \\
\gamma=0}} \varepsilon_{1}\left(\left(\frac{x}{\pi}\right)_{n}\right) \varepsilon_{2}\left(\left(\frac{-x}{\pi}\right)_{n}\right)+\sum_{\substack{\gamma \bmod \pi \\
\gamma \neq 0}} \sum_{\substack{\bmod \pi \\
\pi}} e(\gamma) \varepsilon_{1}\left(\left(\frac{x}{\pi}\right)_{n}\right) \varepsilon_{2}\left(\left(\frac{\gamma-x}{\pi}\right)_{n}\right) \\
& =\varepsilon_{2}\left(\left(\frac{-1}{\pi}\right) \sum_{x \bmod \pi} \varepsilon_{1} \varepsilon_{2}\left(\left(\frac{x}{\pi}\right)_{n}\right)+\sum_{\substack{\gamma \bmod \pi \\
\gamma \neq 0}} \sum_{\gamma x \bmod \pi} \mathbf{e}(\gamma) \varepsilon_{1}\left(\left(\frac{\gamma x}{\pi}\right)_{n}\right) \varepsilon_{2}\left(\left(\frac{\gamma-\gamma x}{\pi}\right)_{n}\right)\right. \\
& =\sum_{\substack{\gamma \bmod \pi \\
\gamma \neq 0}} \varepsilon_{1} \varepsilon_{2}\left(\left(\frac{\gamma}{\pi}\right)_{n}\right) \mathbf{e}(\gamma) \sum_{x \bmod \pi} \varepsilon_{1}\left(\left(\frac{x}{\pi}\right)_{n}\right) \varepsilon_{2}\left(\left(\frac{1-x}{\pi}\right)_{n}\right) \\
& =\mathfrak{g}\left(r, \varepsilon_{1} \varepsilon_{2}, \pi\right) J\left(\varepsilon_{1}, \varepsilon_{2}, \pi\right) .
\end{aligned}
$$

For the module of $J\left(\varepsilon_{1}, \varepsilon_{2}, \pi\right)$, we use the known formula for the module of the Gauss sums:

$$
\left|J\left(\varepsilon_{1}, \varepsilon_{2}, \pi\right)\right|=\frac{\left|\mathfrak{g}\left(r, \varepsilon_{1}, \pi\right)\right|\left|\mathfrak{g}\left(r, \varepsilon_{1}, \pi\right)\right|}{\left|\mathfrak{g}\left(r, \varepsilon_{1} \varepsilon_{2}, \pi\right)\right|}=\frac{N_{K / \mathbb{Q}}(\pi)^{1 / 2} N_{K / \mathbb{Q}}(\pi)^{1 / 2}}{N_{K / \mathbb{Q}}(\pi)^{1 / 2}}=N_{K / \mathbb{Q}}(\pi)^{1 / 2}
$$


Remark Suppose that $c=\pi^{k}$ with $k>1$ then

$$
\left(\frac{x}{\pi^{k}}\right)_{n}=\left(\frac{x}{\pi}\right)_{n}^{k}
$$

and is never a primitive character on $G\left[\pi^{k}\right]$ and therefore $\mathfrak{g}\left(r, \varepsilon_{1}, \pi^{k}\right)=0$. This generalizes to

$$
\mathfrak{g}\left(r, \varepsilon_{1}, \alpha\right)=0 \quad \text { if } \quad \mu(\alpha)=0,
$$

where $\alpha$ is any algebraic integer, coprime to $r$ and $\mu(\alpha)$ is the Möbiüs function on $K$. For this reason, a much more generalized theorem of Eisenstein $[\mathbf{E}]$ and Weil $[\mathbf{w} 2],[\mathbf{w} 3]$ is as follows:

Theorem 3.6. Let $\varepsilon_{1}, \varepsilon_{2}$ be two homomorphisms of $\mu_{n}(K) \rightarrow \mu_{n}(\mathbb{C})$. Then there exists a Grössencharakter $\omega\left(\varepsilon_{1}, \varepsilon_{2}, c\right)$ of $K$ such that, if $c$ is coprime to $r$ and $n$ then

$$
\mathfrak{g}\left(r, \varepsilon_{1}, c\right) \mathfrak{g}\left(r, \varepsilon_{2}, c\right)=\mu(c) \omega\left(\varepsilon_{1}, \varepsilon_{2}, c\right) \mathfrak{g}\left(r, \varepsilon_{1} \varepsilon_{2}, c\right) .
$$

Where $\mu$ is the Möbius function in $K$.

We therefore see that if $\varepsilon$ is a generator of order $n$ of the group of complex characters of $\mu_{n}(K)$, then

$$
\begin{gathered}
\mathfrak{g}(r, \varepsilon, c) \mathfrak{g}(r, \varepsilon, c)=\mu(c) \omega(\varepsilon, \varepsilon, c) \mathfrak{g}\left(r, \varepsilon^{2}, c\right) . \\
\mathfrak{g}(r, \varepsilon, c) \mathfrak{g}\left(r, \varepsilon^{2}, c\right)=\mu(c) \omega\left(\varepsilon, \varepsilon^{2}, c\right) \mathfrak{g}\left(r, \varepsilon^{3}, c\right) . \\
\ldots \\
\ldots \\
\mathfrak{g}(r, \varepsilon, c) \mathfrak{g}\left(r, \varepsilon^{n-1}, c\right)=\mathfrak{g}(r, \varepsilon, c) \mathfrak{g}\left(r, \varepsilon^{-1}, c\right) \\
=\mu(c) \omega\left(\varepsilon, \varepsilon^{n-1}, c\right) \mathfrak{g}\left(r, \varepsilon^{n}, c\right)=\mu(c) \varepsilon\left(\frac{-1}{c}\right)_{n}|\mathfrak{g}(r, \varepsilon, c)|^{2}=\mu(c) \varepsilon\left(\frac{-1}{c}\right)_{n} N_{K / \mathbb{Q}}(c) .
\end{gathered}
$$

It then follows that

$$
\mathfrak{g}(r, \varepsilon, c)^{n}=\mu(c)^{n} \varepsilon\left(\frac{-1}{c}\right)_{n} N_{K / \mathbb{Q}}(c) \omega\left(\varepsilon, \varepsilon^{2}, c\right) \omega\left(\varepsilon, \varepsilon^{3}, c\right) \cdots \omega\left(\varepsilon, \varepsilon^{n-2}, \pi\right)
$$

Now let us go back to the fifth cyclotomic field and state the following lemma:

Lemma 3.4. If $\pi$ is a prime element of $\mathbb{Z}\left[\zeta_{5}\right]$ dividing the prime $p \in \mathbb{Z}$ and let $\chi$ be a primitive Dirichlet character modulo $\pi$ of order 5 , then $\rho=\pi \sigma_{3}(\pi)$ is an associate of the Jacobi sum $J(\chi, \chi)$. More precisely, $\rho= \pm \zeta^{j} J(\chi, \chi)$, for some integer $i$ between 0 and 4 . Moreover if $\pi$ is primary, then $\rho= \pm J(\chi, \chi)$.

Proof. By the uniqueness of the factorization of $p$ in $\mathbb{Z}[\zeta]$, any $\alpha$ in $K / K^{+}$ whose norm is $p$ has the form $\alpha=u \cdot \sigma_{i}(\pi) \sigma_{j}(\pi)$ where $u$ is a unit of $K$ and $i, j$ are integers between 1 and 4 , with $i \not \equiv \pm j \bmod 5$. Hence $\alpha=u \sigma_{k}(\rho)$ for a unique $k$, $1 \leq k \leq 4$. Also since

$$
|\alpha|=\left|\sigma_{k}(\rho)\right|=\sqrt{p}
$$

we have $|u|=1$ and for all $\mathbb{Q}$-conjugates $\sigma_{j}(u)$ of $u,\left|\sigma_{j}(u)\right|=1$, therefore $u$ is a root of unity in $K$ by the Kronecker theorem; i.e $u= \pm \zeta^{i}$. Let $\chi_{0}$ be another Dirichlet 
character modulo 5 , then put $\alpha=J\left(\chi_{0}, \chi_{0}\right)$. We know that $N\left(J\left(\chi_{0}, \chi_{0}\right)\right)=\sqrt{p}$, then $\rho= \pm \zeta^{i} \sigma_{j}\left(J\left(\chi_{0}, \chi_{0}\right)\right)$; i.e

$$
\rho= \pm \zeta^{i} J\left(\chi_{0}^{j}, \chi_{0}^{j}\right)
$$

for unique integers $0 \leq i \leq 4,1 \leq j \leq 4$. We then conclude by taking $\chi=\chi_{0}^{j}$.

Now we suppose that $\pi$ is primary, therefore $\rho \equiv 1 \bmod (1-\zeta)^{3}$ for all $\mathrm{j}$, since $\pi$ and $\sigma_{j}(\pi)$ satisfies the same requirements. If $g$ is a primitive root modulo $p$ such that $\chi(g)=\zeta^{h}$, with $h \in \mathbb{Z}$. For any $m \in \mathbb{Z}$ prime to $p$ we denote $i n d_{g}(m)$ the index of $m$ with respect to $g$. Then

$$
\begin{aligned}
J(\chi, \chi) & =\sum_{t=2}^{p-1} \zeta^{h\left(i n d_{g}(t)+i d_{g}(1-t)\right)} \\
& \equiv \sum_{t=2}^{p-1}\left(1-h\left(i n d_{g}(t)+i d_{g}(1-t)\right)\right)(1-\zeta) \\
& \equiv p-2+h\left(2 \sum_{k=1}^{p-2} k\right)(1-\zeta) \bmod (1-\zeta)^{3} \\
& \equiv-1 \bmod (1-\zeta)^{3}, \text { since } \quad p-1 \equiv 0 \bmod (1-\zeta)^{4} .
\end{aligned}
$$

Since

$$
\rho= \pm \zeta^{i} J(\chi, \chi) \equiv \pm(1-i(1-\zeta))(-1) \bmod (1-\zeta)^{3} \equiv 1 \bmod (1-\zeta)^{3}
$$

implies that $i \equiv 0 \bmod 5$ and therefore $\rho= \pm J(\chi, \chi)$.

\section{The moment Gauss sums and the uniform distribution}

Here the notations differ slidely from the previous, but they are essentially the same. The field we consider here is of the form $K=\mathbb{Q}\left(\zeta_{q-1}\right)$ where $q$ is of the form $p^{f}$ and $n \mid q-1$. A consequence of this fact is that $\mathbb{Q}\left(\zeta_{n}\right) \subset K$.

We define $\varepsilon$ as before as an injective homomorphism

$$
\varepsilon: \mu_{q-1}(K) \rightarrow \mu_{q-1}(\mathbb{C}) .
$$

We furthermore suppose $\varepsilon$ is an isomorphism.

Then $\chi=\chi_{\pi}$ is a Dirichlet character modulo $\pi$ so that $\varepsilon \circ \chi(x)$ has values in $\mathbb{C}$. We also put

$$
\mathbf{e}(x)=\exp \left(2 \pi \operatorname{Tr}_{K / \mathbb{Q}}(x)\right)
$$

where $\operatorname{Tr}_{K / \mathbb{Q}}$ is the trace of $K / \mathbb{Q}$. We then reformulate the Gauss sum as follow:

$$
\mathfrak{g}(a, \varepsilon \circ \chi, \pi)=\sum_{x} \varepsilon(\chi(x)) \mathbf{e}\left(\frac{a \cdot x}{\pi}\right)
$$

and with this definition, all the properties of Gauss sums are satisfied.

Definition 3.3. Under the assumption above and for any positive integer $m$ we define the $m^{\text {th }}$ moment of the Gauss sum $\mathfrak{g}(a, \varepsilon \circ \chi, \pi)$, as follows:

$$
M_{m} \mathfrak{g}:=M_{m} \mathfrak{g}(a, \varepsilon \circ \chi, \pi)=\sum_{a \neq 0} \sum_{(\bmod \pi)} \mathfrak{x \neq 1} \mathfrak{g}(a, \varepsilon \circ \chi, \pi)^{m}
$$


In the next lines, we are going to give a non trivial estimate of the module $\left|M_{m} \mathfrak{g}\right|$. The trivial estimate is obtained by using the properties involving the module of the Gauss sums.

In fact $|\mathfrak{g}(a, \varepsilon \circ \chi, \pi)| \leq \sqrt{q}$ therefore

$$
\left|M_{m} \mathfrak{g}\right| \leq \sum_{a \neq 0} \sum_{\chi \neq 1}|\mathfrak{g}(a, \varepsilon \circ \chi, \pi)|^{m}=(q-1)(q-2) q^{m / 2}
$$

One can perform this estimate as follows:

Another property of the Gauss sum for a non trivial character states that for any $a \not \equiv 0(\bmod \pi)$,

$$
\mathfrak{g}(a, \varepsilon \circ \chi, \pi)=\varepsilon(\bar{\chi}(a)) \mathfrak{g}(1, \varepsilon \circ \chi, \pi),
$$

and then taking into account the character relation on the residues class modulo $\pi$ we obtain

$$
\begin{aligned}
& \left.\mid \sum_{a} \mathfrak{( m o d} \pi\right) \\
= & 0 \quad \text { if } \quad \chi^{m} \neq 1 \\
= & (q-1) q^{m / 2} \quad \text { if } \quad \chi^{m}=1
\end{aligned}
$$

then the characters with significant effect in the sum are those whose order divides $m$. The number of such chararcters is $d=\operatorname{gcd}(m, q-1)$.

Therefore

$$
\left|M_{m} \mathfrak{g}\right| \leq \sum_{\chi \neq 1}\left|\sum_{a} \mathfrak{g}(a, \varepsilon \circ \chi, \pi)^{m}\right|=\sum_{\substack{\chi^{d}=1 \\ \chi \neq 1}}(q-1) q^{m / 2}=(d-1)(q-1) q^{m / 2} .
$$

We summarize this result in the following theorem:

Theorem 3.7. Let $m \geq 1$, and let $d=\operatorname{gcd}(m, q-1)$, the greatest common divisor of $m$ and $q-1$. Then we have

$$
\left|M_{m} \mathfrak{g}\right| \leq(d-1)(q-1) q^{m / 2}
$$

The next step consists of the equidistribution properties of the arguments of the Gauss sums.

In fact

$$
|\mathfrak{g}(a, \varepsilon \circ \chi, \pi)|=\sqrt{q}
$$

implies that

$$
\frac{\mathfrak{g}(a, \varepsilon \circ \chi, \pi)}{\sqrt{q}}
$$

lies on the unit circle in the complex plane. It is important to consider the equidistribution properties of the following sequence in the interval $[0,1]$ as $q \rightarrow \infty$

$$
\frac{1}{2 \pi} \arg (\mathfrak{g}(a, \varepsilon \circ \chi, \pi))
$$

whenever $\pi \bigwedge a$ and $\chi \neq 1$. Here $\arg (z)$ stands for the argument of the complex number $z$.

We want to establish the equidistribution estimate for the above sequence with a 
good error term.

Let $\alpha_{1}$ and $\alpha_{2}$ be any real numbers with $0 \leq \alpha_{1}<\alpha_{2} \leq 1$ and let

$$
L\left(\alpha_{1}, \alpha_{2}\right):=\#\left\{(a, \chi): \pi \not a, \quad \chi \neq 1 \quad \text { and } \quad \alpha_{1} \leq \frac{1}{2 \pi} \arg (\mathfrak{g}(a, \varepsilon \circ \chi, \pi)) \leq \alpha_{2}\right\}
$$

We are now looking for an estimate of $L\left(\alpha_{1}, \alpha_{2}\right)$. We first recall the Erdös-Turan discrepancy theorem $[\mathbf{K u N i}]$ pp. 112 on the uniform distribution. If $\left\{a_{m}\right\}$ is a sequence of reals in the interval $[0,1]$. Then for any positive integer $N$, denote the discrepancy

$$
D_{N}=\sup _{0 \leq \delta_{1}<\delta_{2} \leq 1} \mid\left(\frac{1}{N}\left(\sum_{\substack{1 \leq m \leq N \\ \delta_{1} \leq a_{m} \leq \delta_{2}}} 1\right)-\left(\delta_{1}-\delta_{2}\right) \mid .\right.
$$

for any positive integer $K$, from $[\mathbf{M O N}]$ pp. 8, the estimate for $D_{n}$ is as follows:

$$
N D_{N} \leq \frac{N}{K+1}+3 \sum_{1 \leq m \leq K} \frac{1}{m}\left|\sum_{1 \leq j \leq N} e^{2 \pi i m a_{j}}\right|
$$

Therefore replace $N$ by $(q-1)(q-2)$ in $D_{N}$ and use the definition of $L\left(\delta_{1}, \delta_{2}\right)$ we get

$$
\begin{aligned}
& \left|L\left(\delta_{1}, \delta_{2}\right)-(q-1)(q-2)\left(\delta_{1}-\delta_{2}\right)\right| \\
\leq & \frac{(q-1)(q-2)}{K+1}+3 \sum_{1 \leq m \leq K} \frac{1}{m q^{m / 2}}\left|\sum_{\chi \neq 1_{a \neq 0}} \mathfrak{g}(a, \varepsilon \circ \chi, \pi)^{m}\right| \\
\leq & \frac{(q-1)(q-2)}{K+1}+3 \sum_{1 \leq m \leq K} \frac{\left|M_{m} \mathfrak{g}\right|}{m q^{m / 2}}
\end{aligned}
$$

we take $K=q-1$ and then using the estimate for $M_{m} \mathfrak{g}$ we deduce that

$$
\begin{gathered}
\left|L\left(\delta_{1}, \delta_{2}\right)-(q-1)(q-2)\left(\delta_{1}-\delta_{2}\right)\right| \\
\leq(q-2)+3(q-1) \sum_{1 \leq m \leq q-1} \frac{\operatorname{gcd}(m, q-1)-1}{m} .
\end{gathered}
$$

We give now an estimate to the sum $\sum_{1 \leq m \leq q-1} \frac{\operatorname{gcd}(m, q-1)}{m}$. In fact

$$
\begin{aligned}
\sum_{1 \leq m \leq q-1} \frac{\operatorname{gcd}(m, q-1)}{m} & =\sum_{n \mid q-1} \sum_{\substack{1 \leq m \leq(q-1) / n \\
\operatorname{gcd}(m,(q-1) / n)=1}} \frac{1}{m} \\
& \leq(1+\log q) \sum_{n \mid q-1} 1=(1+\log q D(q-1)) \\
\leq 2 D(q-1) \log q &
\end{aligned}
$$

where $D(n)$ is the number of positive divisor of $n$.

Thus we finally have 


$$
\left|L\left(\delta_{1}, \delta_{2}\right)-(q-1)(q-2)\left(\delta_{1}-\delta_{2}\right)\right| \leq 7 q D(q-1) \log q .
$$

We can now state the theorem as follows:

Theorem 3.8. we have

$$
\left|L\left(\delta_{1}, \delta_{2}\right)-(q-1)(q-2)\left(\delta_{1}-\delta_{2}\right)\right| \leq 7(q-1) D(q-1) \log q
$$

Remark The $m$-moment of the Gauss sum would certainly be simplify if we introduce the properties from the Davenport-Hasse theorem. At least it would give a much better result in term of congruences modulo $n$ the order of the character $\chi$. This is not the place to undertake that. Both theorems would certainly lead to alike ones in the theory of Jacobi sums, since both sums are related. 


\section{CHAPTER 4}

\section{CYCLOTOMIC CRYSTAL}

\section{Introduction}

We denote by $K$ an algebraic number field of finite degree over $\mathbb{Q}$ and we assume $K$ contains $\mu_{n}$, the group of the $n^{\text {th }}$ roots of unity. Let $V=K \otimes_{\mathbb{Q}} \mathbb{R}$ ( as algebra, $\left.V \cong \mathbb{R}^{N}, N=\operatorname{dim}_{\mathbb{Q}}(K)\right)$ and regard $V$ as a topological algebra, that is, $V$ is also a linear space. For $\mathrm{K}=\mathbb{Q}\left(\mu_{n}\right)$, the space $V$ is also viewed as the infinite component of the adéle ring $K_{\mathbb{A}}$ of $K$ and $N$ is the absolute degree of $K$.

Definition 4.1. (1) The mapping

$$
z \otimes x \mapsto(\zeta z) \otimes x
$$

for $\zeta \in \mu_{n}, z \in K, x \in \mathbb{R}$ is called a rotation( the rotation by $\zeta$ ).

(2) The mapping $z \mapsto z+b(b \in \mathfrak{R}$ the ring of integers of $K)$ is the translation by $b$.

(3) For $a \in K-\{0\}$ and $b \in \mathfrak{R}$, a transformation $\sigma(a, b)$ is the mapping $z \mapsto a z+b$

Definition 4.2. Let

$$
\Gamma=\left\{\sigma(a, b) ; a \in \mu_{n}, b \in \mathfrak{R}\right\}
$$

We call $\Gamma$ a cyclotomic crystallographic group. To see that $\Gamma$ is a group, we define the composition as follows

$$
\sigma(a, b) \sigma(c, d)=\sigma(a c, a d+b)
$$

and

$$
\sigma(a, b)^{-1}=\sigma\left(a^{-1},-a^{-1} b\right)
$$

with this definition, $\Gamma$ is a discrete group which acts discontinuously on $V$; furthermore, $\Gamma$ is isomorphic to $\mu_{n} \rtimes \mathfrak{R}$.

In general, there is a fundamental domain of a crystallographic group which is a polyhedron. This is the case of our group $\Gamma$, where the fundamental domain is given by parallelotopes. Here, a parallelotope is the generalization of a higher dimensional parallelogram. In general it is not always possible to obtain a fundamental domain of $\Gamma$ by a single parallelotopes, but we need a finite number of them. This construction of the fundamental domain is very useful in the geometric point of view in the algebraic number theory. one uses it to find absolute residue system for an ideal $\mathfrak{a}$ of $\mathfrak{R}$ and one derives a generalization of the Gauss lemma which leads to the definition of the Legendre symbol, and therefore, give another proof to the reciprocity law. ( $[\mathbf{R}-\mathbf{H}]$, Kubota ). The idea behind it is that it can also help in determining Euclidean algorithm for certain number fields. This will appear in this 
chapter throughout as a consequence of some combinatoric facts. We give a complete description of the skeleton of the fundamental domain for cyclotomic fields of prime order.

Our investigation on the geometric aspect of the fundamental domain for $\Gamma$ leads to some combinatorial results for certain values of $n$, precisely when $n$ is an odd and prime. We shall also derive for $n=5$, and for general $n$ an interesting property concerning the Euclidean property of the corresponding field. The geometric definition of the fundamental domain for $\Gamma$ is that of Dirichlet. For, one needs in general to endow the space $V$ with some quadratic function $d$, positive definite. Let $d$ to be any norm function defined on $V$, which preserved by $\Gamma$, that is

$$
d(\gamma x, \gamma y)=d(x, y)
$$

for all $x, y \in V$ and $\gamma \in \Gamma$.

Definition 4.3. See [A. Bear] pp 204.

A fundamental set for $\Gamma$ is a subset $D$ of $V$ which contains exactly one point from each orbit in $V$. Thus no two points in $D$ are $\Gamma$-equivalent and

$$
\bigcup_{\gamma \in \Gamma} \gamma F=V
$$

Definition 4.4. See [A. Bear] pp 204.

A subset $F$ of the metric space $(V, d)$ is a fundamental domain for the group $\Gamma$ if and only if

- (1) $F$ is a domain.

- (2) There is some fundamental set $D$ with $F \subset D \subset \bar{F}$.

- (3) The $d-\operatorname{vol}(\partial(F))=0$

Remark If $F$ is a fundamental domain, then for $\gamma \in \Gamma(\gamma \neq I, \mathrm{I}$ is the identity element of $\Gamma$ ),

$$
(\gamma F) \cap F=\emptyset, \quad \bigcup_{\gamma \in \Gamma} \gamma \bar{F}=V
$$

Definition 4.5. (Dirichlet fundamental domain.)

Let $x \in V$ fixed. Then

$$
F_{x}=\{y: d(x, y)<d(\gamma x, y), \text { for all } \gamma \in \Gamma\}
$$

is the Dirichlet fundamental domain centered at $x$.

We denote $F_{0}$ by $F$.

The set $\Gamma$, which is a subgroup of transformations of $V$ preserving $d$ is of the form $\operatorname{Ort}_{d} \times V$, where $\mathrm{Orth}_{d}$ is the orthogonal group associated with the quadratic form defining $d$. The stabilizer of a point is compact. It follows that the set:

$$
\{\gamma, \quad \gamma \in \Gamma: d(x, y)=d(x, \gamma y) \text { for } y \in \bar{F}\}
$$

is compact and discrete in Orth $_{d} \times V$ and therefore finite. $V=\Gamma F$, thus for all $x \in V$ there exists $\gamma \in \Gamma$ and $y \in F$ with $x=\gamma y$. 
Theorem 4.1. There exists a finite number of elements $\gamma_{1}, \cdots, \gamma_{m}$ of $\Gamma$ such that $\overline{F_{x}}$ can be given by a finite number of inequalities

$$
d(x-y) \leq d\left(\gamma_{i} x-y\right) \quad i=1, \cdots, m .
$$

ProOF. See [I.M. Gel]pp 8-9.

Remark This theorem is well known, but it is sometimes hard to find the minimal set for the $\gamma_{i}^{\prime} s$. In finding this minimal set, one can describe the geometry of the fundamental domain for a fixed metric. One notices that different metrics will give different shape of the fundamental domain even if they are equivalent metrics. We will explicitely solve this problem for the cyclotomic fields generated by a primitive $n^{\text {th }}$ root of unity, for $n$ prime and odd.

\section{The cyclotomic case}

In the case of number fields, we have said that $V$ is an algebra such that we can impose some linear conditions on its basis elements. In fact is not necessary to use the algebraicity of $V$ for that, since any linear relation correspond to equivalence class with respect to the corresponding hyperplane.

Let $\zeta$ be a primitive $n^{\text {th }}$ root of unity. The field $K=\mathbb{Q}(\zeta)$ is the $n^{\text {th }}$ cyclotomic field.

Let $e_{i}$ denote the element $\zeta^{i} \otimes 1$ in $K \otimes_{\mathbb{Q}} \mathbb{R}$. Then $\sum_{i=1}^{n} e_{i}=0$, we obtain a map:

$$
\mathbb{R}^{n} \rightarrow V: \quad\left(x_{1}, x_{2}, \cdots, x_{n}\right) \mapsto \sum_{i=1}^{n} x_{i} e_{i}
$$

with kernel $W=\mathbb{R}(1,1, \cdots, 1)$. The dual space to $\mathbb{R}^{n} / W$ is the subspace

$$
\left\{\underline{x}=\left(x_{1}, x_{2}, \cdots, x_{n}\right) \in \mathbb{R}^{n}: \quad x_{1}+x_{2}+\cdots+x_{n}=0\right\}
$$

and $V \cong \mathbb{R}^{n} / W$.

We define the quadratic form

$$
q(\underline{x})=\sum_{i<j}\left(x_{i}-x_{j}\right)^{2} \quad \text { on } \quad \mathbb{R}^{n} / W .
$$

We transfer this quadratic form to $V$ and called it $d$.

Then for $x \in V ; x=\sum_{i=1}^{i=n} x_{i} e_{i}$ where $x_{i} \in \mathbb{R}$ for all $i$

$$
d(x)=\sum_{1 \leq i<j \leq n}\left(x_{i}-x_{j}\right)^{2}=\frac{1}{2} \sum_{i, j=1}^{n}\left(x_{i}-x_{j}\right)^{2}=n \sum_{i=1}^{n} x_{i}^{2}-\left(\sum_{i=1}^{n} x_{i}\right)^{2},
$$

$(V, d)$ is a quadratic space and the corresponding bilinear symmetric form is

$$
(, \quad): V \times V \rightarrow \mathbb{R} ; \quad(x, y) \mapsto \frac{1}{2}[d(x+y)-d(x)-d(y)]
$$

The Dirichlet fundamental domain for $d$ is

$$
F=\left\{x \in V:|(x, y)| \leq \frac{1}{2} d(y), y \in L\right\}
$$

$F$ is a compact convex symmetric subset of $V$.

The aim of our work here is to find a suitable finite set of points such that $F$ is 
the convex hull of these points. This set is chosen to be minimal in such a way that its elements are exactly the vertices of $F$. We will also give a complete combinatoric description of its geometric structure. We prove that this combinatoric structure is related to certain numbers. The symmetric group plays a key role. The Euler relation for polyhedron is satisfied.

The following properties hold:

Lemma 4.1. $\quad$ (1) $\left(e_{i}, e_{i}\right)=n-1, \quad 1 \leq i \leq n$.

- (2) $\left(e_{i}, e_{j}\right)=-1, \quad 1 \leq i \neq j \leq n$.

- (3) $\sum_{i=1}^{n} x_{i} e_{i}=0 \Leftrightarrow x_{i}$ constant for all $i, 1 \leq i, j \leq n$.

- (4) For $x \in V$, there exists a representation of $x$ in the form $x=\sum_{i=1}^{n} x_{i} e_{i}$ with $x_{i} \geq 0$ for all $i$.

- (5) Let $x=\sum_{i=1}^{n} x_{i} e_{i} \in V$ be an integral point, there exists a representation of $x$ of the form $x=\sum_{i=1}^{n} \lambda_{i} e_{i}$ and $0 \leq \sum_{i=1}^{n} \lambda_{i}<n$;

Proof. (1),(2) and (3) are clear.

(4). If all the $x_{i} \geq 0$ then, we are done, otherwise, let $z_{i}=x_{i}+\lambda \geq 0$ where $\lambda \geq \max \left\{\left|x_{i}\right|, 1 \leq i \leq n\right\}$ is any real number. Then $x=\sum_{i=1}^{n} z_{i} e_{i}$.

(5) Put $\lambda=\left[\frac{1}{n} \sum_{i=1}^{n} x_{i}\right]-1$ the $x=\sum_{i=1}^{n}\left(x_{i}-\lambda\right) e_{i}$

Definition 4.6. - For $A \subset N=\{1,2, \cdots, n\}$ an element $v_{A}=\sum_{i \in A} e_{i}$ is called an elementary vector.

- $x_{1}, x_{2} \in V$ are said to be equivalent if they are congruent modulo the subspace generated by $(1,1, \cdots, 1)$

Lemma 4.2. Let $N$ be as before, and $A, B \subset N$. The notation $A^{C}$ stands for $N-A$, the complementary of $A$ in $N$, then:

- (1) $e_{N}=0$

- (2) $e_{A}+e_{B}=e_{A \cup B}+e_{A \cap B}$

- (3) $e_{A \cup B}=e_{A-B}+e_{A \cap B}+e_{B-A}$

- (4) $e_{A^{C}}=-e_{A}$

- (5) $\left(e_{A}, e_{A}\right)=|A|(n-|A|)$

- (6) $\left(e_{A}, e_{B}\right)=-|A||B|$ if $A \subset B^{C}$

- (7) $\left(e_{A}, e_{B}\right)=n|A \cap B|-|A||B|$

- (8) $\left.\left(e_{A}, e_{A \cup B}\right)=|A|(n-|A|)-|A||B|+|A||A \cap B|\right)$

- (9) $\left(e_{A \cap B}, e_{A \cup B}\right)=\left(e_{A}, e_{B}\right)+|B-A||A-B|$

- (10) $\left(v, e_{A}\right)=-\left(v, e_{A^{C}}\right)$ for $v \in V$

Proof. (1),(2),(3),(4) fall from the definition of the elementary vectors and the Morgan's laws. 
(5). From the definition of the quadratic form,

$$
\left(e_{A}, e_{A}\right)=\sum_{i \in A} \sum_{j \in A}\left(e_{i}, e_{j}\right)=\sum_{i \in A}(n-|A|)=|A|(n-|A|) .
$$

(6). Since $A \subset B^{C},\left(e_{i}, e_{j}\right)=-1$ for $i \in A$ and $j \in B$, therefore

$$
\left(e_{A}, e_{B}\right)=\sum_{i \in A} \sum_{j \in B}\left(e_{i}, e_{j}\right)=\sum_{i \in A}(-|B|)=-|A||B|
$$

(7). We decompose the sets $A$ and $B$ as follow

$$
A=(A \cap B) \cup(A-B) \text { and } B=(B \cap A) \cup(B-A) \text {. }
$$

then

$$
\begin{aligned}
\left(e_{A}, e_{B}\right) & =\left(e_{A \cap B}+e_{A-B}, e_{A \cap B}+e_{B-A}\right) \\
& =n|A \cap B|-|A \cap B|(|A-B|+|B-A|)-|A-B||B-A| \\
& =n|A \cap B|-|A \cap B|(|A|+|B|-|A \cap B|) \\
& -(|A|-|A \cap B|)(|B|-|B \cap A|) \\
& =n|A \cap B|-|A||B|
\end{aligned}
$$

The (8) is a direct consequence of (7).

The (9). From (7) we have on the one hand

$$
\begin{aligned}
\left(e_{A \cap B}, e_{A \cup B}\right) & =n|A \cap B|-|A \cap B||A \cup B| \\
& =n|A \cap B|-|A \cap B|(|A|+|B|-|A \cap B|)
\end{aligned}
$$

and on the other hand, we have

$$
\begin{aligned}
\left(e_{A}, e_{B}\right)+|A-B||B-A| & =n|A \cap B|-|A||B|+(|A|-|A \cap B|)(|B|-|A \cap B|) \\
& =n|A \cap B|-|A \cap B|(|A|+|B|-|A \cap B|)
\end{aligned}
$$

The (10), follows from (1).

Theorem 4.2. Let $y \in F$ with $d(y)$ maximal.

Let $S=\left\{A \subset N:\left(x, e_{A}\right)=\frac{1}{2} d\left(e_{A}\right)\right\}$

Then $S$ is totally ordered under inclusion.

Proof. $N \in S$ and therefore $S$ is not empty .

Let $A, B \in S$. We have to prove that either $A \subset B$ or $B \subset A$

Put $C=A-B, D=B-A$, then

if $D=\emptyset$ or $C=\emptyset$ we are done.

Otherwise, we have $C \neq \emptyset \neq D$ 
Since $A, B \in S$ we have $\left(x, e_{A}\right)=\frac{1}{2} d\left(e_{A}\right)$ and $\left(x, e_{B}\right)=\frac{1}{2} d\left(e_{B}\right)$

$(2.10)\left(x, e_{A}+e_{B}\right)=\frac{1}{2}\left(d\left(e_{A}\right)+d\left(e_{B}\right)\right)$

$$
\begin{aligned}
& =\left(x, e_{A \cap B}\right)+\left(x, e_{A \cup B}\right) \text { by lemma } 4.2(2) \\
& =\frac{1}{2} d\left(e_{A}+e_{B}\right)-\left(e_{A}, e_{B}\right) \text { because } d \text { is a quadratic form }
\end{aligned}
$$$$
>\frac{1}{2}\left(d\left(e_{A \cap B}+e_{A \cup B}\right)\right)-\left(e_{A \cap B}, e_{A \cup B}\right) \text { by lemma } 4.2(9)
$$$$
=\frac{1}{2} d\left(e_{A \cap B}\right)+\frac{1}{2} d\left(e_{A \cup B}\right)
$$

Therefore

$$
\left(x, e_{A \cap B}\right)+\left(x, e_{A \cup B}\right)>\frac{1}{2} d\left(e_{A \cap B}\right)+\frac{1}{2} d\left(e_{A \cup B}\right)
$$

implies that at least one of the following inequalities holds:

$$
\left(x, e_{A \cap B}\right)>\frac{1}{2} d\left(e_{A \cap B}\right)
$$

or

$$
\left(x, e_{A \cup B}\right)>\frac{1}{2} d\left(e_{A \cup B}\right)
$$

Which contradicts the fact that $x \in F$, and hence

$x \in F$ implies that $(C=\emptyset$ or $D=\emptyset)$ so that it follows that $(A \subset B$ or $B \subset B)$

Remark We know that the fundamental domain is defining by a finite set of inequalities. By proving that $F=F_{1}$, we can explicitely determine all the inequalities since we know all the $e_{A}$ 's.

Theorem 4.3. We have:

$$
F=\left\{x \in V:\left(x, e_{A}\right) \leq \frac{1}{2} d\left(e_{A}\right), \quad \emptyset \neq A \subset N\right\}
$$

Proof. Let $F_{1}$ be the set defined in the statement of the theorem. By the definition of $F$, we have $F \subset F_{1}$; thus the only inclusion to be proved is that $F_{1} \subset F$. Let $y \in L$ so that $y$ is not an elementary vector. Since we are considering lattice points, we can introduce a recurrence relation on the function $d$. From the lattice point $y$, we consider the neighboring points of the form $y \pm e_{i}$. Then for $y \in L$ and $y \neq e_{A}, A \in N$ there exists an element $u= \pm e_{i} \in L$ such that $d(u)+d(y-u)<d(y)$. To see that, take

$$
y=\sum_{i=0}^{n} m_{i} e_{i}, \quad m_{i} \in \mathbb{Z} \text { and } 0 \leq \sum_{i=0}^{n} m_{i}<n .
$$

This is always possible since $\sum_{i=1}^{i=n} e_{i}=0$.

Then

$$
(y, u)-(u, u)=\frac{1}{2}(d(y)-d(u)-d(y-u))= \pm\left(n m_{i}-\sum_{j=1}^{n} m_{j}\right)-(n-1)
$$


i.e $\frac{1}{2}(d(y)-d(u)-d(y-u))= \pm\left(n m_{i}-\sum_{j=1}^{n} m_{j}\right)-(n-1)$. This expression has no constant sign, precisely it is positive for at least one value of $i$. Suppose that for all $i$

$$
\pm\left(n m_{i}-\sum_{j=1}^{n} m_{j}\right)-(n-1)<0
$$

is negative; then

- (1)

$$
n m_{i} \leq \sum_{j=1}^{n} m_{j}-(n-1) \leq 2 n-2<2 n
$$

implies that $m_{i}<2$ for all $i$.

- $(2)$

$$
n m_{i} \geq \sum_{j=1}^{n} m_{j}-(n-1) \geq-n+1>-n
$$

implies that $m_{i}>-1$ for all $i$.

From (1) and (2), follows that $m_{i} \in\{0,1\}$, and hence $y$ is an $e_{B}$ for some $B \subset N$, which contradicts our assumption that $y$ is not an elementary vector.

Let $x \in F_{1}$ then we have to prove that $x \in F$. This is done by induction as follows. We know that $\left(x, e_{A}\right) \leq \frac{1}{2} d\left(e_{A}\right)$ for all $A \subset N$. Let $y \in L$. There exists u such that $d(u)+d(y-u)<d(y)$ and $u= \pm e_{i}$ for some $i$.

Suppose first that $y-u=e_{A}$ and $y \neq e_{B}$ for all $B \subset N$, and $u$ is solution to the inequality $d(u)+d(y-u)<d(y)$. In this case we have:

$$
(x, y)=\left(x, e_{A}+u\right)=\left(x, e_{A}\right)+(x, u) \leq \frac{1}{2}\left(d\left(e_{A}\right)+d(u)\right)<\frac{1}{2} d(y),
$$

since $x \in F_{1}$. Consequently

$$
(x, y)<\frac{1}{2} d(y)
$$

by the choice of $u$. Hence in this case the inequalities corresponding to $y$ which define $F$ are satisfied. Next, suppose that $y-u$ is not of the form $e_{A}$.

Suppose $y \in L$ and suppose that for all $z$ with $d(z)<d(y)$, the inequality $(x, z)<$ $\frac{1}{2} d(z)$ is satisfied. Then we can write $y=z+u$ with $d(z)+d(u)<d(y)$. In this case

$$
(x, y)=(x, z)+(x, u)<\frac{1}{2}(d(z)+d(y))
$$

since $z$ satisfies $d(z)<d(y)$. Therefore the inequality

$$
(x, y)<\frac{1}{2} d(y)
$$

follows as a consequence.

Example. For $n=3, N=\{1,2,3\}$ and since $e_{1}+e_{2}+e_{3}=0$

Then $F=\left\{x \in V: 2\left|\left(x, e_{i}\right)\right| \leq d\left(e_{i}\right), i=1,2,3\right\}$

$F$ is defined by 6 inequalities.

To describe $F$ fully, we have to determine all its vertices, so that $F$ is nothing else but the convex hull of its vertices. 


\section{The vertices}

Put $b=\max \{d(x) ; x \in \bar{F}\}$.

Then the value $b$ exists and is attained because of the continuity property of $d$ on the one hand, and on the other hand because $F$ is compact.

Lemma 4.3. Let $x_{0} \in V$ and $d\left(x_{0}\right)=b$ then $x_{0}$ is a vertex of $F$.

Proof. see [?]in The geometry of discrete groups.

Theorem 4.4. Let $S=\left\{A \subset N:\left(x_{0}, e_{A}\right)=\frac{1}{2} d\left(e_{A}\right)\right\}$

The span of $\left\{e_{A}, A \in S\right\}$ over $\mathbb{R}$ has dimension $n-1$ and possesses a basis : $\left\{e_{A_{i}}, 0<i \leq n-1\right\}$ with $\emptyset \neq A_{i} \varsubsetneqq A_{i+1} \varsubsetneqq N$.

Proof. Suppose that $W$, the $\mathbb{R}$-span of $\left\{e_{A}, A \in S\right\}$ has codimension $\geq 1$. Then for some suitable $u \in V-\{0\}$, we have $\left(u, e_{A}\right)=0$ for all $A \in S$. Then without lost of generality we can suppose $\left(u, x_{0}\right) \geq 0$ otherwise we multiply $u$ by -1 .

By replacing $u$ by $t u$, for real $t$ small enough, we can consider $w \in V$ such that $w=x_{0}+u$ is in some neighborhood of $x_{0}$ with the property that

$$
0 \leq\left(w, e_{B}\right)<\frac{1}{2} d\left(e_{B}\right) \text { for } B \notin S
$$

. Hence we can take $w=x_{0}+u$ with the conditions above, so that for $B \notin S$,

$\left(w, e_{B}\right)=\left(x_{0}+u, e_{B}\right) \leq \frac{1}{2} d\left(e_{B}\right), \quad$ implies that: $\left(u, e_{B}\right) \leq \frac{1}{2} d\left(e_{B}\right)-\left(x_{0}, e_{B}\right) \leq d\left(e_{B}\right)$ for $B \subset N$ and $B \notin S$.

However, if $B \subset N$ then $\left(w, e_{B}\right)=\left(x_{0}+u, e_{B}\right) \leq \frac{1}{2} d\left(e_{B}\right)$.

That implies that for $A \subset N$ we have $x_{0}+u \in F$ by the definition of $F$. But $\left(x_{0}, u\right)>0$ and so

$$
\begin{array}{r}
d\left(x_{0}+u\right) \geq d\left(x_{0}\right)+d(u)+2\left(x_{0}, u\right)>d\left(x_{0}\right) \\
\text { and from this follows that } x_{0}+u \notin F
\end{array}
$$

by the defining property of $x_{0}$. This implies $u=0$.

The linear span of $\left\{e_{A}, A \in S\right\}$ is therefore of dimension $n-1$. We conclude that there are $n-1$ different proper subsets $A_{i}$ such that $\left\{e_{A_{i}}, 0<i<n ; A_{i} \in S\right\}$ forms a basis of $V$.

If $A, B \in S$ then

$$
\left(x_{0}, e_{A}\right)=\frac{1}{2} d\left(e_{A}\right) \text { and }\left(x_{0}, e_{B}\right)=\frac{1}{2} d\left(e_{B}\right)
$$

then by the above lemma, this implies that either $A \subset B$ other $B \subset A$ and hence $S$ is totally ordered.

Remark Indeed we can deduce that the vertex $x_{0}$ is the unique solution to the linear equations

$$
\left(x, e_{B}\right)=\frac{1}{2} d\left(e_{B}\right)
$$

for exactly $n-1$ elements $B$ of $S$. 
Remark Since $S$ is totally ordered, $A_{1} \varsubsetneqq A_{2} \varsubsetneqq \cdots \varsubsetneqq A_{n-1} \varsubsetneqq N$.

The set $A_{i}$ must contain at least $i$ elements. and since all of them are proper subsets, $A_{i}$ has exactly $i$ elements. There are many ways of classifying such a sequence, but we will choose one of them in that we define

$$
A_{i}=\{i+1, i+2, \cdots, n\} \quad 1 \leq i \leq n-1
$$

and the corresponding elementary vector

$$
e_{A_{i}}=\sum_{j=i+1}^{n} e_{j}
$$

Theorem 4.5. The vertices of $F$ are the points

$$
x_{\sigma}=\frac{1}{n} \sum_{i=1}^{n} \sigma(i) e_{i}
$$

where $\sigma$ is a permutation of $N$. In particular there are $n$ ! vertices.

Proof. Before we start the proof, we make the following remark. If $\sigma$ is a permutation of $\{1,2, \cdots, n\}$ then the action of $\sigma$ on $A_{i}$ is done elementwise, so that $A_{i}^{\sigma}=B_{i}$ and $\left|A_{i}\right|=\left|B_{i}\right|$. For this reason we consider at most only one chain $A_{1} \varsubsetneqq A_{2} \varsubsetneqq \cdots \varsubsetneqq A_{n}=N$ of subsets of $N$ up to a permutation. Furthermore, if we consider $x_{0}=\sum_{i=1}^{n} \frac{i}{n} e_{i}$ then put $x_{0}^{\sigma}=\sum_{i=1}^{n} \frac{\sigma(i)}{n} e_{i}$, such that $x_{\sigma}=x_{0}^{\sigma}$. We deduce that

$$
\left(x_{0}, e_{A_{i}}\right)=\frac{1}{2} d\left(e_{A_{i}}\right) \quad \text { implies } \quad\left(x_{0}^{\sigma}, e_{A_{i}^{\sigma}}\right)=\left(x_{\sigma}, e_{A_{i}^{\sigma}}\right)=\frac{1}{2} d\left(e_{A_{i}^{\sigma}}\right)
$$

for any permutation $\sigma$.

The proof of the theorem reduces to any particular case. In fact if

$$
\left(x_{0}, e_{A_{i}}\right)=\frac{1}{2} d\left(e_{A_{i}}\right), \quad \text { then } \quad \sum_{j=i+1}^{n}\left(x_{0}, e_{j}\right)=\frac{1}{2} i(n-i) .
$$

We define explicitely the components of $x_{0}$.

Let $x_{0}=\sum_{i=1}^{n} x_{i} e_{i}$ we have $\left(x_{0}, e_{i}\right)=n x_{i}-\sum_{j=1}^{n} x_{j}$ so that if we suppose $\sum_{i=1}^{n} x_{i}=0$ then $\left(x_{0}, e_{j}\right)=n x_{j}$.

This implies $\sum_{j=i+1}^{n} n x_{j}=i(n-i)$ for $0 \leq i \leq n-1$.

Since

$$
\sum_{k=i+1}^{n}\left(k-\frac{n+1}{2}\right)=i(n-i)
$$

then

$$
\sum_{k=i+1}^{n} n x_{k}=\sum_{k=i+1}^{n}\left(k-\frac{n+1}{2}\right) .
$$

Put $n x_{i}=i-\frac{1}{2}(n+1)$ then $x_{i}=\frac{2 i-n-1}{2 n}$ and it follows that

$$
x_{0}=\sum_{i=1}^{n} \frac{2 i-n-1}{2 n} e_{i}=\frac{1}{n} \sum_{i=1}^{n} i e_{i}
$$


since

$$
\sum_{i=1}^{n} e_{i}=0
$$

and

$$
b=d\left(x_{0}\right)=\sum_{0<i<j \leq n} \frac{(i-j)^{2}}{n^{2}}=\frac{n^{2}-1}{12}=d\left(x_{0}^{\sigma}\right)
$$

Example. For $n=3$ the situation is as follows.

The generating system is $\left(e_{1}, e_{2}, e_{3}\right)$ with $e_{1}+e_{2}+e_{3}=0$. $N=\{1,2,3\}=\{i, j, k\}$. The proper subsets are

$$
\{1\},\{2\},\{3\},\{1,2\},\{1,3\},\{2,3\} \text {. }
$$

It follows that $e_{i}+e_{j}=-e_{k}$ and $e_{i} e_{j}=e_{i+j}$. The index is taken modulo 3 .

$$
\mathfrak{S}_{3}=\{(1,2,3),(2,1,3),(3,2,1),(1,3,2),(3,1,2),(3,2,1)\}
$$

The vertices are

$$
\begin{aligned}
& \text { Vert }_{3}=\left\{\frac{1}{3}\left(e_{1}+2 e_{2}+3 e_{3}\right), \frac{1}{3}\left(2 e_{1}+e_{2}+3 e_{3}\right), \frac{1}{3}\left(3 e_{1}+2 e_{2}+e_{3}\right)\right. \\
&\left.\frac{1}{3}\left(e_{1}+3 e_{2}+2 e_{3}\right), \frac{1}{3}\left(3 e_{1}+e_{2}+2 e_{3}\right), \frac{1}{3}\left(3 e_{1}+2 e_{2}+e_{3}\right)\right\} \\
&=\left\{-\frac{1}{3}\left(2 e_{1}+e_{2}\right),-\frac{1}{3}\left(e_{1}+2 e_{2}\right), \frac{1}{3}\left(2 e_{1}+e_{2}\right), \frac{1}{3}\left(-e_{1}+e_{2}\right)\right. \\
&\left.\frac{1}{3}\left(e_{1}-e_{2}\right), \frac{1}{3}\left(2 e_{1}+e_{2}\right)\right\}
\end{aligned}
$$

3.1. Generating integral points from the vertices. We just have to show how one can construct $e_{1}, \cdots, e_{n}$ from the vertices, since they generate the $\mathbb{Z}$-module of integral elements.

Let $e_{j}, \quad 1 \leq j \leq n$ and $\sigma, \tau$ are permutations of $\{1,2, \cdots, n\}$ such that $\sigma(j)=n$, $\tau(i)=n-\sigma(i)$ if $i \neq j$ and $\tau(j)=n$, then define

$$
v_{j}=\sum_{i=1}^{n} \frac{\sigma(i)}{n} e_{i} \quad \text { and } \quad v_{j}^{*}=\sum_{i=1}^{n} \frac{\tau(i)}{n} e_{i} .
$$

$$
\text { It follows that } e_{j}=v_{j}+v_{j}^{*} \text {. }
$$

This solution is not unique. However an integral point can be represented as

$$
\alpha=\sum_{i=1}^{n} m_{i} e_{i}=\sum_{i=1}^{n} m_{i}\left(v_{i}+v_{i}^{*}\right)
$$


4. THE GEOMETRY AND THE COMBINATORIC OF THE FUNDAMENTAL DOMAIN FOR N = 359

\section{The geometry and the combinatoric of the fundamental domain for $\mathrm{n}=3,5$}

4.0.1. Case $n=3$. In this case, $N=\{1,2,3\}$ so that for $\emptyset \neq A \subset N$, we have $d\left(e_{A}\right)=2$

for $v \in V_{e r t}$ then inequality for the fundamental domain is

$$
F=\left\{x:\left(x, e_{A}\right) \leq \frac{1}{2} d\left(e_{A}\right)=1 \quad \text { for all } \quad \emptyset \neq A \subset N\right\} .
$$

For a fixed $A$ we look at all vertices that is solution to the above equation. It is relevant to notice that $\left(v, e_{A}\right) \in\{-1,0,1\}$. The 0 -dimensional faces described by $\mathrm{A}$, are the solutions to the system of equations

$$
\left\{\begin{array}{l}
\left(v, e_{A}\right)=1 \\
\left(v, e_{B}\right)=0 \text { for } \mathrm{A} \subset \mathrm{B}
\end{array}\right.
$$

and the 1-dimensional faces, are solutions to

$$
\left(x, e_{A}\right)=1 \text { for all } A \subset N \text {. }
$$

The fundamental domain has a very simple shape. It has 6 vertices the so-called 0 -dimensional faces and 61 -dimensional faces, that are segments. This domain is also called the Dirichlet hexagon.

4.0.2. The Euler relations. This relation is taken from the $[\mathbf{W R}]$ pp 127 theor 3.5.1]

Let $\mathrm{P}$ be a non empty $r$-dimensional polytope in $\mathbb{R}^{n}$. Then

$$
f_{-1}(P)-f_{0}(P)+f_{1}(P)+\cdots+(-1)^{r+1} f_{r}(P)=0
$$

Where $f_{k}(P), k \geq 0$ denotes the number of $k$-faces of $P$ and $f_{-1}(P)=1$, hence for $r=3$ one verifies that $1-6+6-1=0$

4.1. Case $\mathbf{n}=\mathbf{5}$. In this case $N=\{1,2,3,4,5\}$ so that for the subset $A$ such that

$$
\emptyset \neq A \subset N, d\left(e_{A}\right)=4
$$

for $v \in \operatorname{Vert}_{5}$, the inequality for the fundamental domain is

$$
\left(x, e_{A}\right) \leq d\left(e_{A}\right)=2 .
$$

The fundamental domain is the convex hull of the vertices

$$
\left\{\sum_{i=1}^{5} \frac{\sigma(i)}{5} e_{i}, \quad \text { with } \sigma \in \mathfrak{S}_{5}\right\}
$$

If $A$ is a proper subset of $N$, there exists at least one $v=\sum_{i=1}^{5} \frac{\sigma(i)}{5} e_{i}$ such that $\left(v, e_{A}\right)=\frac{1}{2} d\left(e_{A}\right)$.

Suppose $v_{0}=\frac{1}{5}\left(e_{1}+2 e_{2}+3 e_{3}+4 e_{4}+5 e_{5}\right)$. For A consists of one element we have

\begin{tabular}{|r|l|l|r|r|r|}
\hline $\mathrm{A}$ & $\{1\}$ & $\{2\}$ & $\{3\}$ & $\{4\}$ & $\{5\}$ \\
\hline$v_{0}, e_{A}$ & -2 & -1 & 0 & 1 & 2 \\
\hline
\end{tabular}

and therefore for $\sigma \in \mathfrak{S}_{5}\left(v_{0}^{\sigma}, e_{A}\right)$ does not lead to change in the range of the set $\{-1,-2,0,1,2\}$. We obtain $v_{0}$ in solving the system of linear equation for $A=\{1\} \subset B=\{1,2\} \subset C=\{1,2,3\} \subset D=\{1,2,3,4\} \subset E=\{1,2,3,4,5\}$. 
Any other permutation $\sigma \in \Sigma_{5}$ on this sequence yields a permutation on the entries such that $F$ is an invariant set and hence any vertex is defined by exactly 5 linear equations.

\section{Remark}

We recall that there are exactly $5 !=120$ vertices

We do not give the proof since it falls from the above description which involves the cardinality of the permutation group, but we will later find this proof in a combinatorial way.

4.1.1. determination of the 3-dimensional faces. From the above description, there are as many vertices as possible sequences of subsets in $N$ and each proper subset determines a linear equation in such a way that the fundamental domain is the intersection of convex half-spaces. A linear equation in $V$ determines a hyperplane and its interaction with $F$ describe a 3-dimensional face of $F$.

From the relation $\sum_{i=1}^{5} e_{i}=0$ we notice that the faces are classified in pairs of parallel faces. It will follow that the lower dimensional faces are paired in a similar fashion.

Lemma 4.4. There are $30=2^{5}-23$ dimensional faces.

Proof. To see that we just notice that each face of $F$ is defined via

$$
\left(x, e_{A}\right)=\frac{1}{2} d\left(e_{A}\right)
$$

, where A is a proper subset of $N$.

They are as many faces as non trivial subsets of $N$; These are

$$
\begin{gathered}
A_{1}=\{1\}, A_{2}=\{1,2\}, A_{3}=\{1,3\}, A_{4}=\{1,4\}, A_{5}=\{1,5\}, A_{6}=\{2\} \\
A_{7}=\{2,3\}, A_{8}=\{2,4\}, A_{9}=\{2,5\}, A_{10}=\{3\}, A_{11}=\{3,4\}, \\
A_{12}=\{3,5\}, A_{13}=\{4\}, A_{14}=\{4,5\}, A_{15}=\{5\}, \\
A_{16}=\{2,3,4,5\}, A_{17}=\{3,4,5\}, A_{18}=\{2,3,5\}, A_{19}=\{2,4,5\} \\
A_{20}=\{2,3,4\}, A_{21}=\{1,3,4,5\}, A_{22}=\{1,4,5\}, \\
A_{23}=\{1,3,5\}, A_{24}=\{1,3,4\}, A_{25}=\{1,2,4,5\}, \\
A_{26}=\{1,2,5\}, A_{27}=\{1,2,4\}, A_{28}=\{1,2,3,5\}, \\
A_{29}=\{1,2,3\}, A_{30}=\{1,2,3,4\} .
\end{gathered}
$$

What is interesting for the combinatorics of this domain, is that we can study completely all the geometric structure using only subsets. That is what we will be doing in the next steps. The notation $F_{3}(A), F_{2}(A), F_{1}(A), F_{0}(A)$ will denote successively the 3-dimensional, 2-dimensional, 1-dimensional and 0-dimensional faces which are contained in the face associated to the underlying subset $A$. 
4. THE GEOMETRY AND THE COMBINATORIC OF THE FUNDAMENTAL DOMAIN FOR N = 35耳

4.1.2. Determination of the 2-dimensional faces. A 2-dimensional face is the intersection of two 3-dimensional faces. Let $v \in F_{A} \cap F_{B}$, Then $v$ satisfies simultaneously

and

$$
2\left(v, e_{A}\right)=d\left(e_{A}\right)
$$

$$
2\left(v, e_{B}\right)=d\left(e_{B}\right)
$$

Then consequently, it means either $A \subset B$ or $B \subset A$. Therefore the intersection of two 3-dimensional faces exists if their underlying subsets satisfy inclusion property. This reduces considerably the testing for the distinct 2-dimensional faces.

For example $F_{3}(\{1,2\}) \cap F_{3}(\{1,5\}), F_{3}(\{1,2\}) \cap F_{3}(\{3,5\})$ are not 2-dimensional faces. One notices that distinct subset with the same cardinality does not satisfy the inclusion property and so their inetersection does not yield a lower dimensional face.

We notice also that if $A \cap B=\emptyset$ then $F_{3}(A)$ and $F_{3}(B)$ are parallel and therefore have no common lower dimensional face.

However, to find the number of different 2-dimensional objects we will replace $\{1,2,3,4,5\}$ by $\{a, b, c, d, e\}$ where each of them can play the same role as the others do. We are going to give different description for $A$ and $B$ and then count the number of different possibilities.

- $A=\{a\}$ and $B=\{a, b\}$ then one has

$$
\left(\begin{array}{l}
5 \\
1
\end{array}\right)\left(\begin{array}{l}
4 \\
1
\end{array}\right)=5 \times 4=20 \text { faces }
$$

- $A=\{a\}$ and $B=\{a, b, c\}$ then one has

$$
\left(\begin{array}{l}
5 \\
1
\end{array}\right)\left(\begin{array}{l}
4 \\
2
\end{array}\right)=\frac{5 \times 4 \times 3}{2}=30 \text { faces }
$$

- $A=\{a\}$ and $B=\{a, b, c, d\}$ then one has

$$
\left(\begin{array}{l}
5 \\
1
\end{array}\right)\left(\begin{array}{l}
4 \\
3
\end{array}\right)=5 \times 4=20 \text { faces }
$$

- $A=\{a, b\}$ and $B=\{a, b, c\}$ then one has

$$
\left(\begin{array}{l}
5 \\
2
\end{array}\right)\left(\begin{array}{l}
3 \\
1
\end{array}\right)=5 \times 6=30 \text { faces }
$$

- $A=\{a, b\}$ and $B=\{a, b, c, d\}$ then one has

$$
\left(\begin{array}{l}
5 \\
2
\end{array}\right)\left(\begin{array}{l}
3 \\
2
\end{array}\right)=30 \text { faces }
$$

- $A=\{a, b, c\}$ and $B=\{a, b, c, d\}$ then one has

$$
\left(\begin{array}{l}
5 \\
3
\end{array}\right)\left(\begin{array}{l}
2 \\
1
\end{array}\right)=20 \text { faces }
$$

Therefore the number of 2-dimensional faces are

$\left(\begin{array}{l}5 \\ 1\end{array}\right)\left(\begin{array}{l}4 \\ 1\end{array}\right)+\left(\begin{array}{l}5 \\ 1\end{array}\right)\left(\begin{array}{l}4 \\ 2\end{array}\right)+\left(\begin{array}{l}5 \\ 1\end{array}\right)\left(\begin{array}{l}4 \\ 3\end{array}\right)+\left(\begin{array}{l}5 \\ 2\end{array}\right)\left(\begin{array}{l}3 \\ 1\end{array}\right)+\left(\begin{array}{l}5 \\ 2\end{array}\right)\left(\begin{array}{l}3 \\ 2\end{array}\right)+\left(\begin{array}{l}5 \\ 3\end{array}\right)\left(\begin{array}{l}2 \\ 1\end{array}\right)=20+30+20+30+30+20=150$

Lemma 4.5. We have shown that the fundamental domain $F$ has 150 2-dimensional faces. 
ProOF.

Now we are going to describe the geometric structure of the 2-dimensional faces. Suppose $\emptyset \neq A \subset B$ to find the number of vertices one has to play with the construction of possible distinct chains from the subchain $A \subset B$

- $\quad A=\{a\}$ and $B=\{a, b\}$ then put $C=\{a, b, c\}$ and $D=\{a, b, c, d\} A \subset B \subset C \subset D$.

there are $\left(\begin{array}{l}1 \\ 3\end{array}\right)=3$ such $C$ and 2 such $D$, therefore there are exactly 6 possible constructions each of which results in uniquely determining a vertex. Such a 2-dimensional face is a hexagon.

$$
A=\{a\} \text { and } B=\{a, b, c\}
$$

in putting $C=\{a, b\}$ and $D=\{a, b, c, d\}$ then $A \subset C \subset B \subset D$.

there are $\left(\begin{array}{l}1 \\ 2\end{array}\right)=2$ such $C$ and 2 such $D$, therefore there are exactly 4 possible constructions each of which results in uniquely determining a vertex. Such a 2-dimensional face is a parallelogram.

$$
A=\{a\} \text { and } B=\{a, b, c, d\}
$$

then put $C=\{a, b\}$ and $D=\{a, b, c\} A \subset C \subset D \subset B$.

there are $\left(\begin{array}{l}1 \\ 3\end{array}\right)=3$ such $C$ and 2 such $D$, therefore there are exactly 6 possible constructions each of which results in uniquely determined a vertex. Such a 2-dimensional face is a hexagon.

- $\quad A=\{a, b\}$ and $B=\{a, b, c\}$ then put $C=\{a\}$ and $D=\{a, b, c, d\}, C \subset A \subset B \subset D$.

there are 2 such $C$ and 2 such $D$, therefore there are exactly 4 possible constructions each of which results in uniquely determined a vertex. Such a 2-dimensional face is a parallelogram.

$$
A=\{a, b\} \text { and } B=\{a, b, c, d\}
$$

then put $C=\{a\}$ and $D=\{a, b, c\}, C \subset A \subset D \subset B$.

there are 2 such $C$ and 2 such $D$, therefore there are exactly 4 possible constructions each of which results in uniquely determined a vertex. Such a 2-dimensional face is a parallelogram.

- $\quad A=\{a, b, c\}$ and $B=\{a, b, c, d\}$

Then the situation is just symmetric if one supposes $A=\{a\}$ and $B=\{a, b\}$ Therefore we still have a hexagon.

- $\quad A=\{a, b, c, d\}$ and $B=\{a\}$

Then the situation is just symmetric if one supposes $A=\{a\}$ and $B=$ $\{a, b, c, d\}$ and we have a hexagon.

\section{Remark}

- We have observed that there are two different types of geometries of the surfaces. They are either hexagon or parallelogram. This idea suggests one that in higher dimension this geometry becomes quiet complicated.

- The following sequence of vertices taken in this order forms a parallelogram: $\frac{1}{5}(5,4,1,3,2), \frac{1}{5}(5,4,3,1,2), \frac{1}{5}(5,4,3,2,1)$, $\frac{1}{5}(5,4,2,3,1), \frac{1}{5}(5,4,2,1,3), \frac{1}{5}(5,4,1,2,3)$, 
4. THE GEOMETRY AND THE COMBINATORIC OF THE FUNDAMENTAL DOMAIN FOR N = 363

4.1.3. Determination of the 1-dimensional faces. A 1-dimensional face is the intersection of two 2-dimensional faces whenever it is not empty. As we have done in the preceeding cases, we are going to evaluate the number of different chains of subsets with 3 components $\emptyset \neq A \subset B \subset C$

- $A=\{a\}$ and $B=\{a, b\}, C=\{a, b, c\}$

their cardinality is

$$
\left(\begin{array}{l}
5 \\
1
\end{array}\right)\left(\begin{array}{l}
4 \\
1
\end{array}\right)\left(\begin{array}{l}
3 \\
1
\end{array}\right)=5 \times 4 \times 3=60
$$

- $A=\{a\}$ and $B=\{a, b\}, C=\{a, b, c, d\}$

their cardinality is

$$
\left(\begin{array}{l}
5 \\
1
\end{array}\right)\left(\begin{array}{l}
4 \\
1
\end{array}\right)\left(\begin{array}{l}
3 \\
2
\end{array}\right)=5 \times 4 \times 3=60
$$

- $A=\{a\}$ and $B=\{a, b, c\}, C=\{a, b, c, d\}$

their cardinality is

$$
\left(\begin{array}{l}
5 \\
1
\end{array}\right)\left(\begin{array}{l}
4 \\
2
\end{array}\right)\left(\begin{array}{l}
3 \\
1
\end{array}\right)=5 \times 6 \times 2=60
$$

- $A=\{a, b\}$ and $B=\{a, b, c\}, C=\{a, b, c, d\}$

their cardinality is

$$
\left(\begin{array}{l}
5 \\
2
\end{array}\right)\left(\begin{array}{l}
3 \\
1
\end{array}\right)\left({ }_{1}^{2}\right)=10 \times 3 \times 2
$$

The total number of $1-$ dimensional faces is

$$
\left(\begin{array}{l}
5 \\
1
\end{array}\right)\left(\begin{array}{l}
4 \\
1
\end{array}\right)\left(\begin{array}{l}
3 \\
1
\end{array}\right)+\left(\begin{array}{l}
5 \\
1
\end{array}\right)\left(\begin{array}{l}
4 \\
1
\end{array}\right)\left(\begin{array}{l}
3 \\
2
\end{array}\right)+\left(\begin{array}{l}
5 \\
1
\end{array}\right)\left(\begin{array}{l}
4 \\
2
\end{array}\right)\left(\begin{array}{l}
3 \\
1
\end{array}\right)+\left(\begin{array}{l}
5 \\
2
\end{array}\right)\left(\begin{array}{l}
3 \\
1
\end{array}\right)\left(\begin{array}{l}
2 \\
1
\end{array}\right)=240 .
$$

4.1.4. Determination of the 0 -dimensional faces. With this method we can determine the 0 -dimensional face, that are vertices, if we consider chains of subsets of length 4 .

$$
A=\{a\} \subset B=\{a, b\} \subset C=\{a, b, c\} \subset D=\{a, b, c, d\}
$$

Their number is

$$
\left(\begin{array}{l}
5 \\
1
\end{array}\right)\left(\begin{array}{l}
4 \\
1
\end{array}\right)\left(\begin{array}{l}
3 \\
1
\end{array}\right)\left(\begin{array}{l}
2 \\
1
\end{array}\right)=5 \times 4 \times 3 \times 2=120=5 !
$$

4.1.5. The Euler relation. Let $\mathrm{P}$ be a non empty 4-dimensional polytope in $\mathbb{R}^{n}$. Then

$$
f_{-1}(P)-f_{0}(P)+f_{1}(P)-f_{2}(P)+f_{3}(P)-f_{4}(P)=0
$$

Where $f_{k}(P)$ denotes the number of $k$-faces of $\mathrm{P}$, then one verifies that

$$
1-120+240-150+30-1=0 .
$$




\subsubsection{The geometric structure of the 3-faces.}

Lemma 4.6. There are two different kinds of a 3-dimensional face. It is either a convex hull of 24 vertices or a convex hull of 12 vertices.

Remark We notice that for a face related to a subset $A$ the symmetry imposed by the complement $A^{C}$ of $A$ yields in determining a symmetric face which of course has the same number of vertices. That is, in fact, the general case within this respect such that the number of non-trivial faces appears as even numbers. Having said that we take $A=\{a\}$ then we determine the vertices of $F_{3}(A)$ in constructing the sequences of length 4 .

$$
A=\{a\} \subset B=\{a, b\} \subset C=\{a, b, c\}, D=\{a, b, c, d\}
$$

Then the dual sequence with respect to the complement results in a symmetric vertex.

Then for $A=\{a\}$ fixed, we have

$$
\left(\begin{array}{l}
4 \\
1
\end{array}\right)\left(\begin{array}{l}
3 \\
1
\end{array}\right)\left(\begin{array}{l}
2 \\
1
\end{array}\right)=24
$$

For $B=\{a, b\}$ we have

$$
\left(\begin{array}{l}
2 \\
1
\end{array}\right)\left(\begin{array}{l}
3 \\
1
\end{array}\right)\left(\begin{array}{l}
2 \\
1
\end{array}\right)=12
$$

We say that a 3 -dimensional object is of type I if the corresponding subset is $A=\{a\}$ or $A=\{a, b, c, d\}$ and of type II if the underlined subset is $A=\{a, b\}$ or $A=\{a, b, c\}$. We now ask the question to know how many surface are there in a 3-dimensional face.

- type I $A=\{a\}$

either $B=\{a, b\}$ or $B=\{a, b, c\}$ or $B=\{a, b, c, d\}$

then we have

$$
\left(\begin{array}{l}
4 \\
1
\end{array}\right)+\left(\begin{array}{l}
4 \\
2
\end{array}\right)+\left(\begin{array}{l}
4 \\
3
\end{array}\right)=4+6+4=14
$$

A 3-face of type I has 14 2-dimensional faces of which we have 8 hexagons and 6 parallelograms.

- type II $A=\{a, b\}$

either $B=\{a\}$ or $B=\{a, b, c\}$ or $B=\{a, b, c, d\}$

then we have

$$
\left(\begin{array}{l}
2 \\
1
\end{array}\right)+\left(\begin{array}{l}
3 \\
1
\end{array}\right)+\left(\begin{array}{l}
3 \\
1
\end{array}\right)=2+3+3=8
$$

A 3-face of type II has 8 2-dimensional faces of which we have 2 hexagons and 6 parallelograms.

Remark For the pictures of these 3-faces, see figure 2 and figure 3 


\section{Generalization}

We suppose that $n$ is a prime number as before and

$$
N=\{1,2, \cdots n\} \text {. }
$$

Then $\{0,1\}^{N}=\{A: A \subset N\}$ is the power set.

Let $K$ be an $\mathbb{R}$-algebra of dimension $n-1$ generated by a basis $\left(e_{1}, e_{2}, \cdots, e_{n}\right)$ subject to the relation $e_{n}=-\sum_{i=1}^{n-1} e_{i}$ and $e_{i} * e_{j}=e_{i+j}$. All indices are taken modulo $n$.

Define on $K$ the quadratic form

$$
d(x)=n \sum_{i=1}^{n} x_{i}^{2}-\left(\sum_{i=1}^{n} x_{i}\right)^{2}=\sum_{i<j}\left(x_{i}-x_{j}\right)^{2},
$$

where $x=\sum_{i=1}^{n} x_{i} e_{i}$.

Define

$$
L=\left\{x=\sum_{i=1}^{n} n_{i} e_{i}, \quad n_{i} \in \mathbb{Z}\right\},
$$

$L$ is a $(n-1)$-dimensional lattice in $K$.

The fundamental domain for $d$ if

$$
F=\{x \in K: d(x) \leq d(x-y) \text { for all } y \in L\}
$$

All the propositions above are still valid in this general case, we are going to prove a general combinatoric properties using the knowledge of certain partition set identities.

We denote by $\mathfrak{F}_{k}, 0<k \leq n-2$ the set of all $k$-dimensional faces of $F$. Let $\mathfrak{S}$ be the set of all strictly monotonic (increasing ) sequence of subsets of $N$.

if $s \in \mathfrak{S}$ then $l(s)$ is number of proper subsets of $s$. It is also called the length of $s$. Taking into account the length of $s \in \mathfrak{S}$ we can form subset $\mathfrak{I}_{l}=\{s: l(s)=l\}, l$ standing for the length of the sequences, we see that

$$
\mathfrak{S}=\bigcup_{0<i<n} \mathfrak{I} i
$$

\section{Theorem 4.6.}

$$
\left|\mathfrak{F}_{n-k-1}\right|=\left|\mathfrak{I}_{k}\right|
$$

Proof. Take first $k=n-1$. Since $n=|N|$, to any sequence of length $n-1$ correspond a sequence of length $n$.

A sequence of length $n-1$ is just a renumbering of $\left\{x_{1}\right\} \subset\left\{x_{1}, x_{2}\right\} \subset \cdots \subset$ $\left\{x_{1}, x_{2}, \cdots, x_{n}\right\}$ where $x_{j} \in\{1,2, \cdots, n\}, 1 \leq j \leq n$. Their cardinality correspond to the number of different permutations of $(1,2, \cdots, n)$ and this corresponds to the number of vertices of $F$, i.e the $x_{\sigma}$ of theorem 17 .

Before we go any further we notice that for $k=1,\left|\mathfrak{F}_{n-2}\right|=2^{n}-2$. However if $A$ and $B$ are disjoint proper sets, then there is no chain containing both $A$ and $B$ and therefore $F_{A} \cap F_{B}=\emptyset$. If $A$ determines uniquely a $(n-2)$ dimensional face of $F$, such that two $(n-2)$ dimensional faces are not disjoint exactly when their corresponding subsets have non empty intersection. This generalizes to disjoint $s_{1}=\left(A_{1} \subset A_{2} \subset \cdots \subset A_{k}\right)$ and $s_{2}=\left(B_{1} \subset B_{2} \subset \cdots \subset B_{k}\right)$ sequences of length $k$ such that none of the $A_{i}$ 'appears in the $B_{i}$ 's and vice versa, then if $A_{1} \cap B_{1}=C \neq \emptyset$ 
then a sequence of $\mathfrak{I}_{k+1}$ is obtaining from anyone of both if we add $C$ to. And this chain obviously corresponds to a face of dimension $(k-2)$ in $\mathfrak{F}_{n-k-2}$. Furthermore if $B$ is a subset of $N$ of length $k$ the number of sequences containing $B$ is $k !(n-k)$ !. But in general

$$
\begin{aligned}
\left|\mathfrak{F}_{n-k-1}\right| & =\sum_{i_{1}=1}^{n-1} \sum_{i_{2}=1}^{n_{1}-i_{1}-1} \cdots \sum_{i_{k}=1}^{n_{k-1}-i_{k-1}-1}\left(\begin{array}{l}
n \\
i_{1}
\end{array}\right)\left(\begin{array}{l}
n_{1}-i_{1} \\
i_{2}
\end{array}\right) \cdots\left(\begin{array}{l}
n_{k}-i_{k-1} \\
i_{k}
\end{array}\right) \\
& =\sum_{i_{1}+\cdots+i_{k}<n} \frac{n !}{i_{1} ! \cdots \cdots \cdot i_{k} !\left(n-\sum_{j=1}^{k} i_{j}\right) !} .
\end{aligned}
$$

where $n_{i}=n-\sum_{k=1}^{i-2} i_{k}$ and $\left(\begin{array}{l}n \\ m\end{array}\right)=0$ if $m>n$

Theorem 4.7. There are exactly $\frac{n-1}{2}$ classes of different non congruent $(n-1)$-faces.

Proof. This theorem is illustrated in the case $n=3$ and $n=5$ by figure 2 and figure 3 respectively.

To prove it, observe that, on one hand, two subsets $A$ and $B$ with the same cardinality have the same number of vertices as solutions to finding different sequences of length $n$ containing $A$. Then replace elements of $A$ by that of $B$ yield a bijective map on the sets of vertices. On another hand, subsets with different cardinalities have of course different number of vertices and consequently determine two different geometries. Finally if $A \subset N$, the underlined face of $A$ and $A^{C}$ have the same geometry since they are parallel and $F$ is a symmetric convex set.

\section{Theorem 4.8.}

$$
\left|\mathfrak{F}_{k}\right|=\sum_{i=0}^{k+1}(-1)^{i}(k+1-i)^{n}\left(\begin{array}{l}
k+1 \\
i
\end{array}\right)
$$

Proof. The formula

$$
\sum_{i_{1}=1}^{n-1} \sum_{i_{2}=1}^{n_{1}-i_{1}-1} \cdots \sum_{i_{k}=1}^{n_{k-1}-i_{k-1}-1}\left(\begin{array}{l}
n \\
i_{1}
\end{array}\right)\left(\begin{array}{l}
n_{1}-i_{1} \\
i_{2}
\end{array}\right) \cdots\left(\begin{array}{l}
n_{k}-i_{k-1} \\
i_{k}
\end{array}\right)
$$

is just

$$
\begin{gathered}
\sum_{i_{1}=1}^{n-1} \sum_{i_{2}=1}^{n_{1}-i_{1}-1} \cdots \sum_{i_{k}=1}^{n_{k-1}-i_{k-1}-1} \frac{n !}{i_{1} ! i_{2} ! \cdots i_{k} !\left(n-i_{1}-\ldots i_{k}\right)} \\
\sum_{i_{0}+i_{1}+\cdots+i_{k}=n}^{n-1} \frac{n !}{i_{0} ! i_{1} ! \cdots i_{k} !}
\end{gathered}
$$

times coefficient of $X^{n}$ in $\left(e^{X}-1\right)\left(e^{X}-1\right) \cdots\left(e^{X}-1\right)(k+1)$ times

$$
\begin{gathered}
=(k+1)^{n}-\left(\begin{array}{l}
k+1 \\
1
\end{array}\right) k^{n}+\left(\begin{array}{l}
k+1 \\
2
\end{array}\right)(k-1)^{n}-\cdots+(-1)^{k}\left(\begin{array}{l}
k+1 \\
k
\end{array}\right) 1^{n} \\
=\sum_{i=0}^{k+1}(-1)^{i}(k+1-i)^{n}\left(\begin{array}{l}
k+1 \\
i
\end{array}\right)
\end{gathered}
$$


where $i_{0}=n-\left(i_{1}+\cdots+i_{k}\right)$

Put

$$
\gamma(n, m)=\sum_{i=0}^{m}(-1)^{i}(m-i)^{n}\left(\begin{array}{l}
n \\
i
\end{array}\right)
$$

and

$$
\Gamma(n, m)=\frac{1}{m !} \gamma(n, m)=\frac{1}{m !} \sum_{i=0}^{m}(-1)^{i}(m-i)^{n}\left(\begin{array}{l}
m \\
i
\end{array}\right)
$$

Then we have $\gamma(n, m)=\left|\mathfrak{F}_{k}\right|$.

\section{Lemma 4.7.}

$$
m^{n}=\sum_{j=1}^{n}\left(\begin{array}{c}
m \\
j
\end{array}\right) j ! \Gamma(n, j)
$$

Proof. Let's take two sets $A$ and $B$ having $n$ and $m$ elements respectively. Every function $f: A \rightarrow B$ can be reviewed as a surjective function if we suitably change its codomain . That is if we consider $f: A \rightarrow f(A)=\{y=f(x), x \in A\} \subset$ $B$. Therefore the total number of functions from $A$ to $B$ equal to $m^{n}$ is also equal to the number of the functions in the set

$$
\left\{f: A \rightarrow \text { Bwith }|f(A)|=\gamma_{j}, \quad j=1,2, \cdots, m\right\} .
$$

These functions are pairwise disjoint. The number of surjection from a set of $n$ elements onto a set of $\gamma_{j} \leq n$ elements is equal to $\gamma(n, j)=j ! \Gamma(n, j)$. Since $j$ elements can be chosen out of the $m$ elements of $B$ in $\left(\begin{array}{l}m \\ k\end{array}\right)$ possible ways, the lemma then follows.

\section{Lemma 4.8 .}

$$
\Gamma(n+1, m)=\Gamma(n, m-1)+m \Gamma(n, m)
$$

ProOF.

With this one can tabulate the $\gamma$ 's and $\Gamma$ 's. Moreover $\left|\mathfrak{F}_{n-k-1}\right|=\gamma(n, k)$

Example This is a table of $\gamma(n, j)$

\begin{tabular}{|r|r|r|r|r|r|r|r|r|}
\hline & 1 & 2 & 3 & 4 & 5 & 6 & 7 & \\
\hline 1 & 1 & & & & & & & \\
\hline 2 & 1 & 2 & & & & & & \\
\hline 3 & 1 & 6 & 6 & & & & & \\
\hline 4 & 1 & 14 & 42 & 24 & & & & \\
\hline 5 & 1 & 30 & 150 & 240 & 120 & & & \\
\hline 6 & 1 & 62 & 540 & 1560 & 1800 & 720 & & \\
\hline 7 & 1 & 126 & 1806 & 8400 & 16800 & 15120 & 5040 & \\
\hline
\end{tabular}


Lemma 4.9. For $n>1$

$$
\sum_{m=1}^{n}(-1)^{m-1}(m-1) ! \Gamma(n, m)=0
$$

Proof. Observe that $k ! \gamma(n, k)$ is the coefficient of $x^{n}$ in $\left(e^{x}-1\right)^{k}$. But

$$
\begin{aligned}
\frac{1}{k !}\left(e^{x}-1\right)^{k} & =\sum_{n=k}^{\infty} \frac{\Gamma(n, k)}{n !} x^{n} \\
(-1)^{k-1}(k-1) ! \frac{\left(e^{x}-1\right)^{k}}{k !} & =\sum_{n=k}^{\infty}(-1)^{k-1}(k-1) ! \frac{\Gamma(n, k)}{n !} x^{n}
\end{aligned}
$$

and therefore$$
\sum_{k=1}^{\infty}(-1)^{k-1} \frac{\left(e^{x}-1\right)^{k}}{k !}=\sum_{k=1}^{\infty} \sum_{n=k}^{n}(-1)^{k-1}(k-1) ! \Gamma(n, k) \frac{x^{n}}{n !}
$$

But

$$
\log \left(1+\left(e^{x}-1\right)\right)=\sum_{k=1}^{\infty} \frac{x^{n}}{n !} \sum_{k=1}^{n}(-1)^{k-1}(k-1) ! \Gamma(n, k)
$$

This implies that

$$
x=\sum_{k=1}^{\infty} \frac{x^{n}}{n !} \sum_{k=1}^{n}(-1)^{k-1}(k-1) ! \Gamma(n, k)
$$

hence for $n>1$

$$
\sum_{k=1}^{n}(-1)^{k-1}(k-1) ! \Gamma(n, k)=0
$$

We now state the well-known result in the combinatorial theory of polytopes, Euler's relation. $\Gamma(n, 0)=(-1)^{n-1}$

\section{Theorem 4.9. The Euler relation}

$$
\sum_{k=0}^{n}(-1)^{k+1} k ! \Gamma(n, k)=0
$$

Proof. We give a very simple proof base on the recurrence relation for $\Gamma(n, m)$. Suppose $\mathbf{n}=\mathbf{2}$ then we have $-(-1)+1-2 ! \Gamma(2,1)=1+1-2=0$ and we are done. Suppose $\mathbf{n}>\mathbf{2}$ then the relation

$$
\Gamma(n+1, m)=\Gamma(n, m-1)+m \Gamma(n, m)
$$

implies that

$$
(-1)^{m} m ! \Gamma(n+1, m)=(-1)^{m} m((m-1) ! \Gamma(n, m-1)+m ! \Gamma(n, m))
$$

then

$$
\sum_{m=1}^{n}(-1)^{m} m ! \Gamma(n+1, m)=\sum_{m=1}^{n}(-1)^{n-1}(m-1) ! \Gamma(n, m)
$$


and finally use the above lemma and the facts that $\Gamma(n, n)=1$ and $\Gamma(n, 0)=$ $(-1)^{n-1}$

Remark We observe that the $\Gamma(n, m)$ 's are increasing in the first instance and then decrease with respect to $m$. It reaches its maximal value at about $\left[\frac{n+1}{2}\right]$. it then easy to see that it has link with the behavior of $m !(n-m)$ !. The symbol $[x]$ stands for the largest integer less than the real number $x$.

\section{Cyclotomy revisited}

Here we are going to describe some implications which have a link to the determination of the fundamental domain. These facts are known and proved by different methods.

Let $K$ be as before a cyclotomic number field. We define the measure $\mu$ on $K$ as follows:

$$
\mu(x)=\sum_{\sigma}|\sigma(x)|^{2},
$$

where $x \in K$ and $\sigma$ runs over the different $\mathbb{R}$-algebra homomorphisms $\sigma: K \rightarrow \mathbb{C}$. We extend $\mu$ to $K \otimes_{\mathbb{Q}} \mathbb{R}$ by $d$ as $d(x)=\operatorname{Tr}_{K / \mathbb{Q}}\left(x x^{-1}\right)$. Here the $x^{-1}$ is suitably defined as $\sigma\left(x^{-1}\right):=\overline{\sigma(x)}$. In fact Gauss used this as metric to prove that the ring $\mathbb{Z}[i]$ is an Euclidean ring. My aim here is to prove that this also works for other cases such as 3, 5, 4, 7 and may also work in many others. Gauss used a classical inequality between the arithmetic mean and the geometric mean of finite number of positive real numbers.

Lemma 4.10. Let $x_{1}, \cdots, x_{n} \in \mathbb{R}$, positive and $\alpha_{1}, \cdots, \alpha_{n}>0$ with $\alpha_{1}+\cdots+\alpha_{n}=1$

Then

$$
x_{1}^{\alpha_{1}} \cdots \cdot x_{n}^{\alpha_{n}} \leq \alpha_{1} x_{1}+\cdots+\alpha_{n} x_{n}
$$

This lemma generalizes the geometric arithmetic mean inequality. This is:

Lemma 4.11. We have

$$
N(x)^{2}=\left(\prod_{\sigma}|\sigma(x)|\right)^{2} \leq\left(\frac{d(x)}{n}\right)^{n}
$$

where $\sigma$ runs over all $n=\frac{1}{2}(K: \mathbb{Q})$ different complex embeddings of $K$ in $\mathbb{C}$ and $N(x)$ is the norm function.

Definition 4.7. An integral domain $\mathrm{R}$ is called Euclidean with respect to a given function $f: R \rightarrow \mathbb{N}$ if $f$ has the following properties:

- $f(\alpha)=0$ if and only if $\alpha=0$

- For all $\alpha, \beta \in R-\{0\}$ there exists a $\gamma \in R$ such that $f(\alpha-\beta \gamma)<f(\beta)$

- For every $\kappa>0$ the set $\{f(\alpha): \alpha \in R, f(\alpha)<\kappa\}$ is finite. We call such an $f$ an Euclidean function on $\mathrm{R}$.

Equivalent definitions are given in $[$ H.W.L]. However certain authors restrict their definition to the first two properties. Here the (absolute) norm function $N$ stands for $f$ and satisfies all the properties. We note that $d_{K / \mathbb{Q}}$ the distance function defined above and that is related to the field $K / \mathbb{Q}$. We have the following proposition: 
Proposition 6.1. Let $K \subset L$ be a number fields, and put $n=(K: \mathbb{Q})$ and

$$
d(\alpha)=\sum|\sigma(\alpha)|^{2} \quad \text { for } \quad \alpha \in L
$$

Then

- (1)

$$
\begin{aligned}
& d_{L}(\alpha)-d_{L}(\alpha-\beta)=(L: K)\left(d_{K}\left(\frac{1}{(K: L)} \operatorname{Tr}_{L / K}(\alpha)-\beta\right)-d_{K}\left(\frac{1}{(L: K)} \operatorname{Tr}_{L / K}(\alpha)-\beta\right)\right) \\
& \text { for all } \alpha \in L, \beta \in K . \\
& \bullet \text { (2) if } L=K\left(\zeta_{m}\right) \text { then } \\
& \qquad d_{L}(\alpha)=\frac{1}{m} \sum_{j=1}^{m} d_{K}\left(\operatorname{Tr}_{L / K}\left(\alpha \zeta_{m}^{j}\right)\right) .
\end{aligned}
$$

ProOF. [F-L]

In fact if

$$
F_{K}=\left\{x \in K: d_{K}(x)<d_{K}(x-\gamma) \text { for all } \gamma \in R\right\}
$$

then $b_{K}=\sup \left\{d_{K}(x): x \in F_{K}\right\}$ and for every $x \in K$ there is a $\gamma \in R$ such that $d_{K}(x-\gamma) \leq b_{K}$. Thus $\mathrm{K}$ is norm Euclidean if $b_{K}<1$ and sometimes $b_{K}=1$ is sufficient.

Definition 4.8. We call $b^{\prime} \in \mathbb{R}$ a usable bound if $b^{\prime} \geq b_{K}$ and if for all $x \in F_{K}$ such that $d(x)=b^{\prime}$ there is a root of unity $\zeta \in R$ and $\gamma \in R$ such that

$$
d(x-\gamma)=d(x-\gamma-\zeta)=b^{\prime} .
$$

We see that every $b^{\prime}>b_{K}$ is a usable bound and therefore if $b^{\prime}$ is a usable bound for $\mathrm{K}$, then $\mathrm{K}$ is norm Euclidean.

Theorem 4.10. Let $\zeta_{m}$ be a primitive $m^{\text {th }}$ root of unity, and $L=K\left(\zeta_{m}\right)$. Then $b_{L} \leq(L: K) b_{K}$, and if $b^{\prime}$ is a usable bound for $K$ then so is $(L: K) b^{\prime}$ for $L$.

We then remark that we have $b_{\mathbb{Q}}=\frac{1}{4}$ as simple exercise. Then we see that any cyclotomic field $K / \mathbb{Q}$ with degree $\varphi(n)$ satisfies the bound condition $b_{K} \leq \frac{\varphi(n)}{4}$ and therefore:

Lemma 4.12. For $n=1,3,4,5,8,12 K=\mathbb{Q}\left(\zeta_{n}\right)$ is norm Euclidean.

Proof. Since $\frac{1}{4}$ is a usable bound for $\mathbb{Q}$ we conclude from the theorem that $\frac{\varphi(n)}{4}$ is a usable bound for any $n$. hence for $\varphi(n) \leq 4, \varphi(n)$ is a usable bound for any $n$.

Remark The case $n=5$ was long time ago proved by Ouspensky, see $[\mathbf{O}]$.

Theorem 4.11. For $n=1,3,4,5,7,8,9,11,12,15,20 \quad K=\mathbb{Q}\left(\zeta_{n}\right)$ is norm Euclidean. 
ProOF. To see this we just have to evaluate $b_{K}=\frac{n^{2}-1}{2}$ for primes $3,5,7,11$ and then use the transition property defining a usable bound for composite number. For $n=20=4 \times 5$

$$
b_{\mathbb{Q}\left(\zeta_{20}\right)}=2^{2} b_{\mathbb{Q}\left(\zeta_{5}\right)}=8=\left(\mathbb{Q}\left(\zeta_{20}\right): \mathbb{Q}\right) .
$$

The same argument applies to $n=15$. Then we see that $b_{K} \leq 1$ for all these values.

Remark . This results are not known from different authors, like [H.W.L], but we don't simply know how to extend the result for larger $m$. We therefore still know very few cyclotomic fields which are norm Euclidean.

6.1. The construction of $n^{\text {th }}$ set. In this part of the paper we intend to provide a very simple way of determining an $n^{\text {th }}$ set.

Definition 4.9. Let $\mathfrak{a}$ be an integral ideal of $\mathbb{Z}\left[\zeta_{n}\right]$ the ring of integers of $\mathbb{Q}\left[\zeta_{n}\right]$ and $\mu_{n}$ be as before the group of the $n^{\text {th }}$ roots of unity. A finite set $\mathrm{S}$ of integer of $\mathbb{Z}\left[\zeta_{n}\right]$ is called a $\frac{1}{n}$-set (or $\frac{1}{n}$-representative system) modulo $\mathfrak{a}$ if the set of $\zeta x,\left(\zeta \in \mu_{n}, x \in\right.$ $\mathrm{S})$ forms together with 0 a complete representative system of residues modulo $\mathfrak{a}$.

We intend to give, in case where $n$ is prime, a simple construction of such an $\mathrm{S}$. We must notice that there is not only one way of constructing an $n$-th set. A very simple way is obtained for example, as follows. For a prime to $n$, take any system of representatives modulo a. Pick up an element $x$, put it in $\mathrm{S}$ and delete all elements which are congruent to $\zeta^{i} x, 1 \leq i<n$ and repeat the process with any non deleted element until all elements are deleted.

The difficulties here are that we can not really make a practical computation. For example, for ideals with the same absolute prime norm one may use the same $n$-th set. But by using the geometry one can fix this in a canonical way such that it does depend on the action of the unit group and therefore depends no more on the ideal itself, but on each representative of it. This enables one to handle practical computation. We then make use of a fundamental domain $\mathrm{F}$ we have constructed so far.

Definition 4.10. Let $F$ be the fundamental domain constructed above, and $\pi$ an algebraic integer, then $F_{\pi}=\pi F \cap \mathbb{Z}\left[\zeta_{n}\right] \otimes_{\mathbb{Z}} \mathbb{R}$ is called a set of residues modulo $\pi$.

$F_{\pi}$ is just a homothetic deformation of $F$ and therefore both have the same geometry. This set of residues was called by Habicht $[\mathbf{H a}]$ "absolute" set of residues modulo $\pi$ in place of "smallest". He used this definition to give a geometric proof of the law of cubic reciprocity.

- We have classified the $(n-2)$-dimensional faces according to the length of the corresponding subset. If $A^{k}$ is the set of all subsets of cardinality $k$, then for $k=n-2$, there are exactly $\frac{n-1}{2}$ different incongruent $(n-2)-$ faces. This is an equivalence relation i.e $A \cong B$ if and only if $|A|=|B|$ or $|A|=n-|B|$. We easily see that the cardinal of $A^{k}=\left(\operatorname{card}\left(A^{n-k}\right)\right)$ is a multiple of $n$, hence in each class $A^{k}$ we choose $2 \frac{\left|A^{k}\right|}{n}$ faces, and for this choice, consider the union of the cones at the origin, each containing the $(n-2)$-dimensional 
face as section. From this point of view we can construct symmetric $\frac{1}{n}$-set as Mc Gettrick, C.R. Matthews did in their Ph. D dissertations. This is sometimes useful, but is not enough to have a $\frac{1}{n}$-sets, we need to know how to count points in it. For a subset of $\mathfrak{D} \subset A^{k}$ of cardinality $2 \frac{\left|A^{k}\right|}{n}$, then for $A \in \mathfrak{D}, F_{n-2}(A)$ has an even number of $F_{n-3}(A)$ then we keep a half of them in $F_{n-2}(A)$ as boundaries, and then consider the section of the cone containing the origin and limited by the remainder from the $F_{n-2}(A)$. We should also make sure that the $F_{n-2}(A)$ 's, by rotation, do not match with any other remaining $F_{n-2}(A), A \notin \mathfrak{D}$. This indeed is very difficult, but since all the faces are defined by linear equations, this amounts to saying that half of the inequalities have to be strict.

- Furthermore we have another description of a $\frac{1}{n}$-set.

See the permutation group $\mathfrak{S}_{n}$ as acting on the set $N$. We have already seen that this action runs over the set of vertices. That is, the natural action of $\mathfrak{S}_{n}$ onto $N$ is transitive and we may write $N$ as a homogeneous space:

$$
N \cong \mathfrak{S}_{n} / \mathfrak{S}_{n-1}
$$

Then we take any of the number in $N$ and consider all neighboring face. Here a neighbor face of $A$ is any subset $B$ containing ( or is contained in ) A. Choose for example $A=\{1\}$ then $A$ has $(n-1)$ ! vertices and $2^{n-1}-2$ neighbors. Then the $n^{\text {th }}$ set must contain only $F_{A}$ as a proper face and half of each of the neighboring faces. We have the defining equation

$$
F_{\frac{1}{n}}=\left\{x \cong\left(x_{1}, \cdots, x_{n-1}, 0\right) \in F: x_{1} \geq 0, \cdots, x_{\frac{n-1}{2}} \geq 0, x_{\frac{n+1}{2}}>0, \cdots, x_{n-1}>0\right\}
$$

Remark We notice that for the first case we have more vertices of the original fundamental domain and many equations than in the second case where we only simple inequalities, and fewer vertices.

6.2. The Gauss Lemma. Another application of the $\frac{1}{n}$-set is the Gauss lemma. Let $\mathfrak{a}$ be an integral ideal of $\mathrm{K}=\mathbb{Q}\left(\zeta_{n}\right)$ prime to $n$ and let $S$ be an $\frac{1}{n}$-set modulo $\mathfrak{a}$. If $\alpha$ is any integer of $\mathrm{K}$ prime to $\mathfrak{a}$, there exists a unique $n^{\text {th }}$ root of unity $\varepsilon(\alpha, x)$ such that

$$
\alpha x \equiv \varepsilon(\alpha, x) x^{\prime} \bmod \mathfrak{a}, \quad \text { with } \quad x^{\prime} \in S \text { for a given } \quad x \in S .
$$

If $\mathfrak{a}=\mathfrak{p}$ is a prime ideal, then the power residue symbol $\left(\frac{\alpha}{\mathfrak{p}}\right)_{n}$ of degree $n$ is defined by

$$
\alpha^{\frac{N(\mathfrak{p})-1}{n}} \equiv\left(\frac{\alpha}{\mathfrak{p}}\right)_{n} \bmod \mathfrak{p}, \quad \text { and is an } n^{\text {th }} \text { root of } 1 .
$$

Here $N(\mathfrak{p})=\left(\mathbb{Z}\left[\zeta_{n}\right]: \mathfrak{p}\right)$. If $\mathfrak{a}$ is not a prime, then the power residue symbol is defined multiplicatively as

$$
\left(\frac{\alpha}{\mathfrak{a}_{1} \mathfrak{a}_{2}}\right)_{n}=\left(\frac{\alpha}{\mathfrak{a}_{1}}\right)_{n}\left(\frac{\alpha}{\mathfrak{a}_{2}}\right)_{n} .
$$


Theorem 4.12. Generalization of the Gauss-Schering Lemma[R-H], $[\mathrm{KB2}]$

$$
\prod_{x} \varepsilon(\alpha, x)=\left(\frac{\alpha}{\mathfrak{p}}\right)_{n}, \quad(x \in S) .
$$

Proof. If $\mathfrak{a}=\mathfrak{p}$ is a prime ideal, then $\prod x=\prod x^{\prime} \bmod \mathfrak{p}$ entails

$$
\prod_{x} \varepsilon(\alpha, x) \equiv \alpha^{\frac{N(\alpha)-1}{n}} \bmod \mathfrak{p} .
$$

The proposition is true by the definition.

If $\mathfrak{a}=\mathfrak{a}_{1} \mathfrak{a}_{2}$ We are going to prove the theorem by induction on the number of ideals factors of $\mathfrak{a}$. Thus let $S_{2}$ be the set of elements of $\mathrm{S}$, which are divisible by $\mathfrak{a}_{1}$, and let $\beta_{1} \in K$ be an integer such that $\left(\beta_{1}, \mathfrak{a}\right)=\mathfrak{a}_{1}$. Then for each $x \in S_{2}$, there exists an integer $\gamma(x) \in K$ such that $\beta_{1} \gamma(x) \equiv x \bmod \mathfrak{a}$, and the set of all $\gamma(x)$ forms a $\frac{1}{n}$-set of residues modulo $\left(\mathfrak{a}_{2}\right)$. Since $\alpha x \equiv \varepsilon(\alpha, x) x^{\prime} \bmod \mathfrak{a}$ yields $\alpha \beta_{1} \gamma(x) \equiv \varepsilon(\alpha, x) \beta_{1} \gamma(x) \bmod \mathfrak{a}$. and consequently $\alpha x \equiv \varepsilon(\alpha, x) \gamma\left(x^{\prime}\right) \bmod \mathfrak{a}_{2}$. We then have $\prod_{x} \varepsilon(\alpha, x)=\left(\frac{\alpha}{\mathfrak{a}_{2}}\right)_{n}, x \in S_{2}$.

consider next $S_{1}=S-S_{2}$. Here we see that $S_{1}$ is the union of norm $\left(\mathfrak{a}_{2}\right)$ sets, each of which is a $\frac{1}{n}$-set of residues system $\bmod \left(\mathfrak{a}_{1}\right)$. Since $\operatorname{norm}\left(\mathfrak{a}_{2}\right) \equiv 1 \bmod (n)$ we have

$$
\prod_{x} \varepsilon(\alpha, x)=\left(\frac{\alpha}{\mathfrak{a}_{1}}\right)_{n}^{N\left(\mathfrak{a}_{2}\right)}=\left(\frac{\alpha}{\mathfrak{a}_{1}}\right)_{n}, x \in S_{1} .
$$




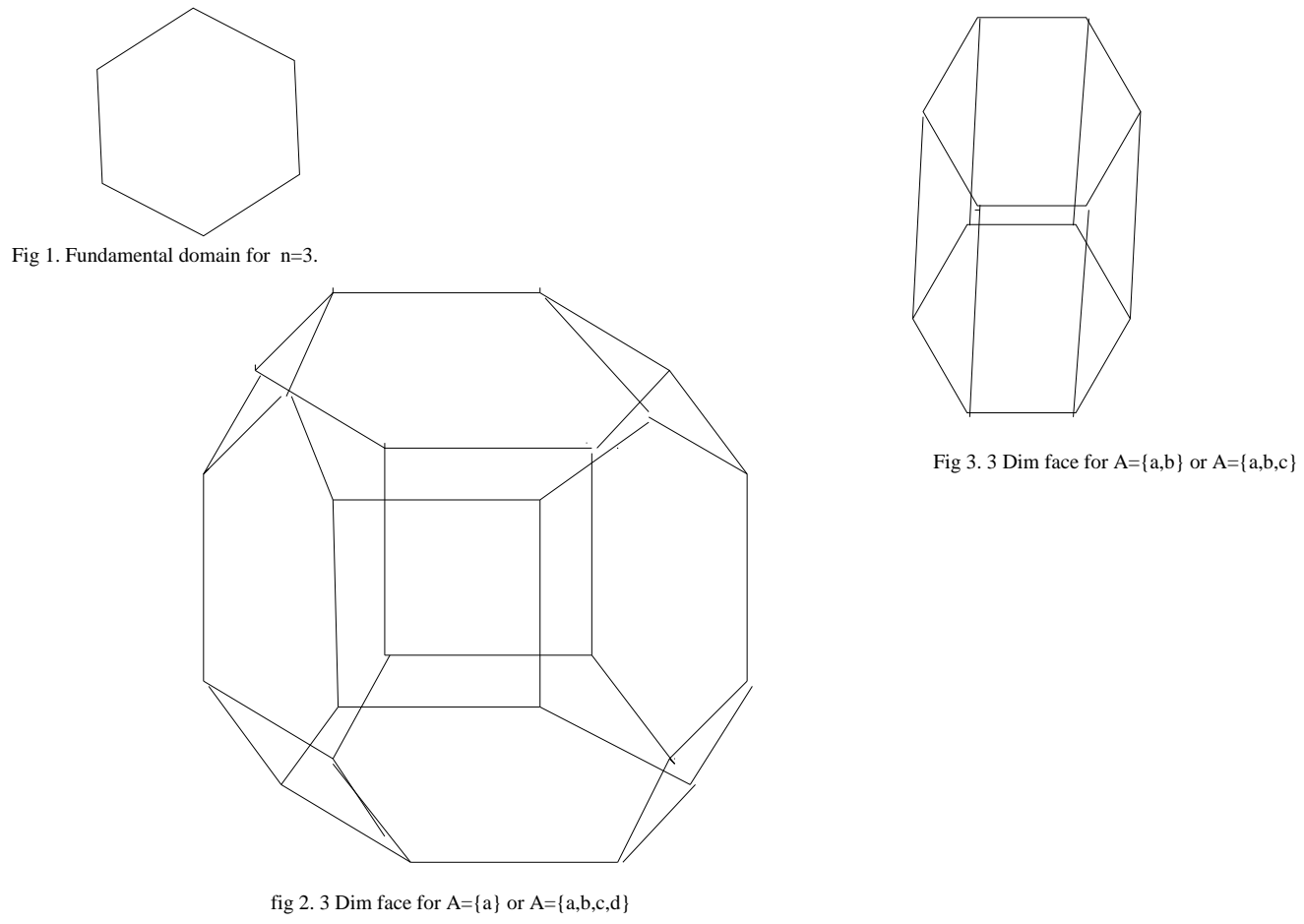

Figure 1. Fundamental domain for $n=3$ and 3 -faces for $n=5$ 


\section{CHAPTER 5}

\section{THE GAUSS SUMS AND THE FUNDAMENTAL DOMAIN}

In this chapter we intend to extend the idea of McGettrick and Cassels in making use of the fundamental domain to find identities linked to the understanding of the Cassels' conjecture. We do not deal explicitely with elliptic functions; we mostly think of that in terms of a 2-dimensional lattice in a quadratic number field. We seek such identities in general algebraic number fields.

Definition 5.1. Let $\Gamma$ be the lattice of the algebraic integers in an algebraic number field $K$. Let $\alpha$ be an integer of $K$. We say that $\beta \in K$ is an $\alpha-$ division point modulo $\Gamma$ if $\alpha \beta \equiv 0 \bmod \Gamma$.

We shall consider henceforth the field $K=\mathbb{Q}(\zeta)$ where $\zeta$ is an $n^{\text {th }}$ root of unity, $n>2$ prime. We consider $\mathbb{Z}[\zeta]$ as a subring of $\mathbb{Q}(\zeta) \otimes_{\mathbb{Q}} \mathbb{R}$.

We have already proved that

$$
F_{\alpha} \cap(\mathbb{Z}[\zeta] \otimes 1)
$$

is a complete set of residues modulo $\alpha$. It has $N_{K / \mathbb{Q}}(\alpha)$ elements. We shall denote this set again by $F_{\alpha}$. This should cause no confusion.

Let $\pi \mid p$ be a prime not dividing $n$ with norm $q$, then a fortiori $n \mid\left(N_{K / \mathbb{Q}}(\pi)-1\right)$, therefore $\mathbb{F}_{q}$ contains an $n^{\text {th }}$ root of unity $\zeta$. If $S_{\pi}$ is any $n^{\text {th }}$ set modulo $\pi$, then:

\section{Theorem 5.1.}

$$
\prod_{x \in S_{\pi}} x=-\zeta_{S_{\pi}}
$$

Where $\zeta_{S_{\pi}}$ is an $n^{\text {th }}$ root of unity depending on $S_{\pi}$

Proof. From Wilson's Theorem, we have

$$
\prod_{x \in F_{\pi}-\{0\}} x \equiv-1 \quad(\bmod \pi)
$$

But since

$$
F_{\pi}=\left(\bigcup_{i=1}^{n} \zeta^{i} S_{\pi}\right) \cup\{0\}
$$

then

$\prod_{x \in F_{\pi}-\{0\}} x=\prod_{x \in S_{\pi}} x \prod_{x \in S_{\pi}} x \zeta \cdots \prod_{x \in S_{\pi}} x \zeta^{n-1}=\zeta^{\frac{(q-1)}{n}(1+2+\cdots+n-1)}\left(\prod_{x \in S_{\pi}} x\right)^{n}=\left(\prod_{x \in S_{\pi}} x\right)^{n}=-1 ;$

then the result follows. 
Remark The construction of $\zeta_{S}$. The $\zeta_{S}$ is constructed such that it satisfies action of the Galois group as the Gauss sum does. Indeed: Let $n$ be an odd prime, an $n^{\text {th }}$ set is of the form

$$
S_{0}=\frac{1}{1-\zeta} F_{0}
$$

where $F_{0}$ is invariant under the Galois group and is contained in $[0,1] e_{1} \times[0,1] e_{2} \cdots \times$ $[0,1] e_{n-1}$ and both have the same volume, for the Lebesgue measure.

For an ideal $\alpha$,

$$
S_{\alpha}=\alpha S_{0}=\frac{\alpha}{1-\zeta} F_{0}
$$

The Galois group operates on $S_{\alpha}$ elementwise. For $g$ a fixed primitive root modulo $n$, and for $\sigma$ a generator of the Galois group such that $\sigma(\zeta)=\zeta^{\sigma}=\zeta^{g}$ and then, this implies that

$$
\sigma\left(S_{\alpha}\right)=\frac{\sigma(\alpha)}{1-\sigma(\zeta)} F_{0}=\left(\sum_{i=0}^{g-1} \zeta^{i}\right)^{-1} \frac{\sigma(\alpha)}{1-\zeta} F_{0} .
$$

therefore $S_{\sigma(\alpha)}=\sum_{i=0}^{g-1} \zeta^{i} \sigma\left(S_{\alpha}\right)$. Let us define

$$
R(\alpha)=\prod_{x \in S_{\alpha}} x \quad(\bmod \alpha)
$$

then

$$
R(\sigma(\alpha))=\left(\frac{\sum_{i=0}^{g-1} \zeta^{i}}{\sigma(\alpha)}\right)_{n} R(\alpha)^{\sigma}
$$

Remark If $g^{\prime}$ is the inverse of the primitive root of $g$ modulo $n$, then replace $\alpha$ by $\sigma^{-1}(\alpha)$ to have

$$
R(\alpha)=\left(\frac{\sum_{i=0}^{g^{\prime}-1} \zeta^{i}}{\alpha}\right)_{n} R\left(\sigma^{-1}(\alpha)\right)^{\sigma}=\left(\frac{\sum_{i=0}^{g^{\prime}-1} \zeta^{i}}{\alpha}\right)_{n} R\left(\sigma^{-1}(\alpha)\right)^{g} .
$$

We easily see that if $g$ is primitive root modulo $n$, then $g^{\prime}$ is also a primitive root modulo $n$ thus we have:

\section{Lemma 5.1. Identity 0}

Let $g$ be any primitive root modulo $n$ and $\sigma: \zeta \mapsto \zeta^{g}$ the element of the Galois group $G$ associated to $g$. Let $\alpha$ be an algebraic integer such that $\alpha \equiv 1 \bmod n$, then we have the following identity:

$$
R(\alpha)=\left(\frac{\sum_{i=0}^{g-1} \zeta^{i}}{\alpha}\right)_{n} R(\sigma(\alpha))^{g^{\prime}} .
$$

Where $g^{\prime}$ is the inverse of $g$ modulo $n$.

Proof. The proof is the consequence of the last identity for $R(\alpha)$.

We observe that since we have $\phi(n-1)$ pairs $\left(g, g^{\prime}\right)$ of primitive roots modulo $n$ we therefore have $\phi(n-1)$ such identities for $R(\alpha)$ 
We notice that there is an extra factor in the formulation of $R(\alpha)$, therefore we will prefer another formulation $\Omega_{0}\left(S_{0}, \alpha\right)$ which avoids this perturbation. furthermore $\Omega_{0}\left(S_{0}, \alpha\right)$ will satisfy complex conjugate transformation, i.e $\Omega_{0}\left(S_{0}, \bar{\alpha}\right)=\overline{\Omega_{0}\left(S_{0}, \alpha\right)}$. This refers to a property of the Gauss sums.

We can sumarize these properties as follows:

$$
\sigma\left(\Omega_{0}\left(S_{0}, \alpha\right)\right)=\Omega_{0}\left(S_{0}, \sigma(\alpha)\right) \quad \text { and } \quad \sigma^{-1}\left(\Omega_{0}\left(S_{0}, \alpha\right)\right)=\Omega_{0}\left(S_{0}, \alpha\right)^{-1} .
$$

Remark : We have to show how to construct $R(\pi)$ set from the above description. It would be invariant under the complex conjugate action. It therefore needs a correction factor. This is due to the fact that the $n$-set attached to $\pi, S_{\pi}$ and the $n$-set attached to $\pi^{\sigma^{-1}}, S_{\pi^{\sigma^{-1}}}$, are not necessary conjugate sets. We give explanations and solutions in the next section.

\section{First identity}

We fix a $n^{\text {th }}$-set $S_{0}$ once and for all, such that $S_{\pi}=\pi S_{0}$.

Let $u$ be a unit in $K=\mathbb{Q}(\zeta) \otimes_{\mathbb{Q}} \mathbb{R}$, if we replace $\pi$ by $u \pi$ then the lemma follows:

Lemma 5.2. The function $R(\pi)$ satisfies for $u$ a unit of $K$;

$$
R(u \pi)=\left(\frac{u}{\pi}\right)_{n} R(\pi)
$$

Proof. Indeed, if $x \in S_{u \pi}$ then $u^{-1} x \in S_{\pi}$

$$
R(\pi)=\prod_{x \in S_{u \pi}} u^{-1} x=u^{-\frac{q-1}{n}} \prod_{x \in S_{u \pi}} x=\left(\frac{u}{\pi}\right)_{n}^{-1} R(u \pi)
$$

The correction factor is done as follows. Before we study a general case, we go through the case $n=5$ first.

Lemma 5.3. Let the prime $\alpha \equiv 1 \bmod 5$ be an algebraic integer in $\mathbb{Z}[\zeta]$ and $S_{\alpha}$ a fifth set modulo $\alpha$, chosen as before. Choose $\sigma$ to be the generator of the Galois group of $\mathbb{Q}[\zeta] / \mathbb{Q}$ such that $\sigma(\zeta)=\zeta^{2}$. If we define

$$
\Omega_{0}\left(S_{0}, \alpha\right)=\left(\frac{\zeta^{2}}{\alpha}\right)_{5} R(\alpha)
$$

then $\Omega_{0}\left(S_{0}, \alpha\right)^{\sigma^{2}}=\Omega_{0}\left(S_{0}, \alpha^{\sigma^{2}}\right)$

Proof. We do this proof only for prime $\pi$ and extend this multiplicatively.

If $\sigma$ maps $\zeta \rightarrow \zeta^{2}$ then $\sigma^{2}$ is the complex conjugate, so that

$x \in \frac{\pi}{1-\zeta} F_{0} \quad$ implies that $\quad x^{\sigma} \in \frac{\pi^{\sigma}}{1-\zeta^{\sigma}} F_{0}^{\sigma}=\frac{\pi^{\sigma}}{1-\zeta^{\sigma}} F_{0}=\frac{\pi^{\sigma}}{1-\zeta^{2}} F_{0} \quad$ since

$F_{0} \quad$ is invariant under the Galois group.

Then from $S_{\pi^{\sigma}}=(1+\zeta) S_{\pi}$ follows that

$$
R\left(\pi^{\sigma}\right) \equiv \prod_{x \in S_{\pi}}(1+\zeta) x^{\sigma} \equiv\left(\frac{1+\zeta}{\pi^{\sigma}}\right)_{5} \prod_{x \in S_{\pi}} x^{\sigma}=\left(\frac{1+\zeta}{\pi^{\sigma}}\right)_{5} R(\pi)^{\sigma} \quad(\bmod \sigma(\pi)) .
$$


In replacing $\pi$ by $\pi^{\sigma}$ we have

$$
R\left(\pi^{\sigma^{2}}\right)=\left(\frac{1+\zeta}{\pi^{\sigma^{2}}}\right)_{5} R\left(\pi^{\sigma}\right)^{\sigma}=\left(\frac{1+\zeta}{\pi^{\sigma^{2}}}\right)_{5}\left(\frac{1+\zeta}{\pi^{\sigma^{2}}}\right)_{5}^{\sigma} R(\pi)^{\sigma^{2}} \quad \text { since } \quad \sigma^{2}
$$

is the complex conjugate map we have

$$
R(\pi) R\left(\pi^{\sigma^{2}}\right)=\left(\frac{1+\zeta}{\pi^{\sigma^{2}}}\right)_{5}\left(\frac{1+\zeta}{\pi^{\sigma^{2}}}\right)_{5}^{\sigma}
$$

On the one hand, we have:

$$
\left(\frac{1+\zeta}{\pi^{\sigma^{2}}}\right)_{5}=\left(\frac{1+\zeta^{\sigma^{2}}}{\pi}\right)_{5}^{-1}=\left(\frac{1+\zeta^{-1}}{\pi}\right)_{5}^{-1}=\left(\frac{1+\zeta}{\pi}\right)_{5}^{-1}\left(\frac{\zeta}{\pi}\right)_{5}
$$

On the another hand, we have:

$$
\left(\frac{1+\zeta}{\pi^{\sigma}}\right)_{5}^{\sigma}=\left(\frac{1+\zeta^{\sigma}}{\pi^{\sigma^{2}}}\right)_{5}=\left(\frac{1+\zeta^{\sigma^{-1}}}{\pi}\right)_{5}^{-1}=\left(\frac{1+\zeta^{-1}}{\pi}\right)_{5}^{-1}=\left(\frac{1+\zeta^{3}}{\pi}\right)_{5}^{-1} .
$$

Therefore if we put

$$
B=\frac{\zeta}{(1+\zeta)\left(1+\zeta^{3}\right)}=\frac{\zeta}{1+\zeta+\zeta^{3}+\zeta^{4}}=-\zeta^{-1} \quad \text { then } \quad R(\pi) R\left(\pi^{\sigma^{2}}\right)=\left(\frac{B}{\pi}\right)_{5} .
$$

If we put $A=\zeta^{2}$ then

$$
A / A^{\sigma^{2}}=-\zeta^{-1} \times u^{5}, \quad \text { where } \mathrm{u} \text { is an algebraic integer. }
$$

and so

$$
R(\pi) R\left(\pi^{\sigma^{2}}\right)=\left(\frac{A}{\pi}\right)_{5}\left(\frac{A}{\pi^{\sigma^{2}}}\right)_{5}
$$

we determine $A$ up to a fifth power. This solution is no way unique but we choose it as simple as possible. For example we take $A=\zeta^{2}$ then we have

$$
\frac{A}{A^{\sigma^{2}}}=\frac{\zeta^{2}}{\zeta^{3}}=-\zeta^{-1}
$$

and with this choice we have

$$
\Omega\left(S_{0}, \pi\right)=\left(\frac{\zeta^{2}}{\pi}\right)_{5} R(\pi)
$$

then the assertion of lemma follows

Remark We notice that the choice for $A=\zeta^{2}$ can be obtained by solving the congruence equation $2 x \equiv-1(\bmod 5)$ which has the solution $x \equiv 2 \bmod 5$. we can generalize it as follows.

Theorem 5.2. Let $p$ and $n$ be prime numbers such that $p \equiv 1 \bmod n$ and $\pi \mid p$ is a prime factor of $p$. Then there exists $m, 0 \leq m \leq n$, so that

$$
\Omega\left(S_{0}, \pi\right)=\left(\frac{\zeta^{m}}{\pi}\right)_{n} R(\pi)
$$


satisfies

$$
\overline{\Omega\left(S_{0}, \pi\right)}=\Omega\left(S_{0}, \pi^{\sigma^{\frac{n-1}{2}}}\right)
$$

Proof. Let $g$ be a primitive root modulo $n$. Then $\sigma: \zeta \rightarrow \zeta^{g}$ generates the Galois group of $\mathbb{Q}\left(\zeta_{q}\right)$.

$$
R\left(\pi^{\sigma}\right)=\left(\frac{1+\zeta}{\pi^{\sigma}}\right)_{n} R(\pi)^{\sigma}
$$

therefore, we obtain inductively

$$
R\left(\pi^{\sigma^{\frac{n-1}{2}}}\right)=\left(\prod_{i=1}^{\frac{n-1}{2}}\left(\frac{1+\zeta^{\sigma^{-} i}}{\pi}\right)_{n}^{-1}\right) R(\pi)^{-1} .
$$

Put

$$
u=\prod_{i=1}^{\frac{n-1}{2}}\left(\frac{1+\zeta^{\sigma^{-}}}{\pi}\right)_{n}^{-1} .
$$

By the definition of the $n^{t h}$ power residue symbol, $u$ is a root of unity i.e $u=\zeta^{k}$, for some $0 \leq k \leq n$.

It follows that

$$
R\left(\pi^{\sigma^{\frac{n-1}{2}}}\right)=\left(\frac{\zeta^{k}}{\pi}\right)_{n}^{-1} R(\pi)^{-1}=\left(\frac{\zeta}{\pi}\right)_{n}^{-k} R(\pi)^{-1} .
$$

We are now looking for a formula $\Omega\left(S_{0}, \pi\right)=\left(\frac{\zeta^{t}}{\pi}\right)_{n} R(\pi)$ which satisfies

$$
\Omega\left(S_{0}, \pi^{\sigma^{\frac{n-1}{2}}}\right)=\overline{\Omega\left(S_{0}, \pi\right)}
$$

as we have done in the case $n=5$, this amount to solving the congruence equation

$$
2 m \equiv-k \quad \bmod n,
$$

since $\mathrm{n}$ is prime and $\operatorname{gcd}(2, n)=1$ we see that this equation has the unique solution $m=-2^{-1} k$ where $2^{-1}$ is the inverse of 2 modulo $n$.

Finally, by combining lemma 5.2 and lemma 5.3, we can sate the first identity as follows.

\section{Identity}

$$
\begin{gathered}
\Omega\left(S_{0}, u \pi\right)=\left(\frac{u}{\pi}\right)_{n} \Omega\left(S_{0}, \pi\right) \\
\Omega\left(S_{0}, \pi^{\sigma^{-1}}\right)=\Omega\left(S_{0}, \pi\right)^{\sigma^{-1}}
\end{gathered}
$$

\section{Second identity}

There is a similar identity with the Gauss sum.

\section{Lemma 5.4.}

$$
\mathfrak{g}(1, \varepsilon, u \pi)=\varepsilon\left(\left(\frac{u}{\pi}\right)_{n}\right) \mathfrak{g}(1, \varepsilon, \pi)
$$


Remark We see that the first and the second identity have the same configuration. Then, if we suppose that the unit $u$ is an $n^{\text {th }}$ power, then the first and second identity are invariant under the unit group $U_{0}^{n}$.

For $n=5$ we have chosen prime $\pi$ to be primary, i.e $\pi \equiv 1 \bmod \lambda^{3}$. We fortunately prove that if $\pi_{1}$ and $\pi_{2}$ are associate primary primes then their ratio is a fifth power unit. The consequence it that in this class of primary primes there is exactly one value of $\mathfrak{g}(1, \varepsilon, u \pi)$ and $\Omega\left(S_{0}, u \pi\right)$. As Patterson suggested to me, if the Gauss sums are to be considered as congruence functions, it would be better to work them in this class of primes.

\section{Third identity}

We will denote the complex root of unity $\varepsilon\left(\Omega\left(S_{0}, \alpha\right)\right)$ by $\Omega\left(S_{0}, \varepsilon, \alpha\right)$.

Lemma 5.5. The function

$$
\frac{\mathfrak{g}(1, \varepsilon, \pi)}{\Omega\left(S_{0}, \varepsilon, \pi\right)}
$$

is independent of the generator of the ideal. It is a function of ideals.

Proof. This follows from the first and second identity.

We extend this formula multiplicatively to obtain

$$
\tilde{g}(1, \varepsilon,(\alpha))=\frac{\mathfrak{g}(1, \varepsilon, \alpha)}{\Omega\left(S_{0}, \varepsilon, \alpha\right)}
$$

for suitable choice of $\alpha$, i.e for example when $N(\alpha) \equiv 1(\bmod n)$

We remark that $\tilde{g}(1, \varepsilon,(\alpha))$ does not depend on the $n^{\text {th }}$ set we choose. In fact if we replace $S_{0}$ by $u S_{0}$ for a unit $u$ this is equivalent to replace $\alpha$ by $u \alpha$.

Remark We then notice that $\tilde{g}(1, \varepsilon,(\alpha))^{n}=\mathfrak{g}(1, \varepsilon,(\alpha))^{n}$. However the $n^{\text {th }}$ power is given by the Stickelberger relations.

Theorem 5.3. Let $(\alpha)$ be a principal ideal in $O_{K}$ which is supposed to be prime to $n$, and let $\varepsilon$ be as before. Then

$$
\mathfrak{g}(1, \varepsilon, \alpha)^{n}=\mu(\alpha)^{n} \chi(\alpha) \varepsilon\left(\alpha^{\sum_{(t, n)=1} t \sigma_{t}^{-1}}\right),
$$

where $\sigma_{t} \in \operatorname{Gal}(K / \mathbb{Q})$ maps $\zeta$ to $\zeta^{t}$ with $\operatorname{gcd}(t, n)=1$ and where $\chi$ is a character of order $n$.

Let us put

$$
\omega(\varepsilon, \alpha)=\varepsilon\left(\alpha^{\sum_{(t, n)=1} t \sigma_{t}^{-1}}\right)
$$

and

$$
\Phi(\alpha)=\mu(\alpha)^{n} \mathfrak{g}(1, \varepsilon, \alpha)^{n}
$$

then, from Weil we know that $\Phi$ is a Grossencharacter with defining ideal $n^{2}$. The Galois group acts on $\phi$ as follows.

$$
\Phi\left(\alpha^{\sigma}\right)=\Phi(\alpha)^{\sigma} .
$$


From this relation we see that

$$
\chi\left(\alpha^{\sigma}\right)=\chi(\alpha)^{\sigma} \text {. }
$$

We want determine $\chi$ explicitly for $n=5$. This result has been suggested by the computation. Let $\lambda=1-\zeta$ and $\tau$ the real fundamental unit $\frac{1+\sqrt{5}}{2}$. we prove that:

Proposition 3.1. Let $\pi$ a prime ideal in $\mathbb{Z}[\zeta]$. $\Phi(\pi)=-\chi(\pi) \varepsilon(\omega(\pi))$ where $\chi(\pi)=1 \quad$ If $\quad \pi \equiv 1 \bmod \lambda^{3}$

$\chi(\tau)=-1$

$\chi(\zeta)=\zeta$

Proof. From Weil, $\chi$ is a character of conductor at most $\lambda^{8}$ where $\lambda=1-\zeta$. Define

$$
\begin{gathered}
U_{k}=\left\{x: \quad x \equiv 1 \bmod \lambda^{k}\right\} . \\
\chi\left(\left(1+a \alpha^{k}\right)\left(1+b \alpha^{k}\right)\right)=\chi\left(1+(a+b) \lambda^{k}\right)
\end{gathered}
$$

Let $\sigma \in \operatorname{Gal}(K / Q)$ such that $\zeta^{\sigma}=\zeta^{2}$ then $\lambda^{\sigma}=1-\zeta^{2}=\lambda(2-\lambda)$

If $\alpha \in U_{8}, \quad \chi(\alpha)=1$.

If $\alpha \in U_{7} \quad$ and $\quad \alpha \notin U_{8}$

then $\chi\left(1+\lambda^{7}\right)^{\sigma}=\chi\left(1+\left(\lambda^{7}\right)^{\sigma}\right)=\chi\left(1+3 \lambda^{7}\right)$ implies that $\chi\left(1+\lambda^{7}\right)^{2}=\chi\left(1+3 \lambda^{7}\right)=$ $\chi\left(1+\lambda^{7}\right)^{3}$ and then follows that $\chi\left(1+\lambda^{7}\right)=1$.

This idea works for $k=6,5,4,3$. For $k=3$, we notice in general that

$$
\left(1+a \lambda^{k}\right) \equiv\left(1+\lambda^{k}\right)^{a} \bmod \left(\lambda^{k+1}\right)
$$

and therefore we obtain

$$
\chi\left(1+\left(\lambda^{3}\right)^{\sigma}\right)=\chi\left(1+2^{3} \lambda^{3}\right)=\chi\left(1+3 \lambda^{3}\right)=\chi\left(1+\lambda^{3}\right)^{3}=\chi\left(1+\lambda^{3}\right)^{\sigma}
$$

and therefore we have $\chi\left(1+\lambda^{3}\right)=1$.

Since $\tau^{\sigma}=-1 / \tau, \quad \chi\left(\tau^{\sigma}\right)=\chi(-1 / \tau)=\chi(-1) \chi(1 / \tau)=\chi(\tau)^{2}$. This implies that $\chi(\tau)^{3}=\chi(-1)=-1$. It follows that $\chi(\tau)=-1$.

From $\Phi(\zeta)=1=\chi(\zeta) \zeta^{\theta}=\chi(\zeta) \zeta^{4}$ follows that $\chi(\zeta)=\zeta$.

This suggests that we can give a simple formulation of the $\Phi(\pi)$ and of course of $\Phi(\alpha)$ whenever it is defined.

Lemma 5.6. For $m=5$

$$
\mathfrak{g}(1, \varepsilon, \pi)^{5}=\mu(\pi)\left(\frac{-\operatorname{Tr}_{K / \mathbb{Q}}(\pi)}{5}\right)_{2} \chi\left(\pi / \pi^{*}\right) \pi^{\theta}
$$

where $\pi^{*}$ is primary associate of $\pi$.

$$
\begin{gathered}
\chi(\pi)=1 \text { if } \pi \equiv 1 \bmod \lambda^{3} \\
\chi(\tau)=-1 \\
\chi(\zeta)=\zeta
\end{gathered}
$$

Proposition 3.2. Let $\pi$ be a prime integer in $\mathbb{Q}(\zeta)$ and $\pi^{*}$ any primary associate of $\pi$ such that $\pi^{*} \equiv 1 \bmod (1-\zeta)^{3}$, then

$$
\Phi(\pi)=\chi\left(\pi^{*} / \pi\right)^{-1} \Phi\left(\pi^{*}\right)=\chi\left(\pi^{*} / \pi\right)^{-1}\left(\pi^{*}\right)^{\theta}
$$




\section{Cassels' formula}

4.1. The Cassels conjecture. The Cassels conjecture is related to the cubic and quartic case of Gauss sums. We can state the cubic case as follows.

The elliptic curve

$$
y^{2}=4 x^{3}-1
$$

over the complex numbers is parameterized by the Weierstrass function

$$
x=\wp(z), \quad y=\wp^{\prime}(z)
$$

with periods

$$
\theta \mathbb{Z}[\zeta]
$$

where $\theta=3.0599087$.. is the smallest positive real period. Then for prime $\pi \mid p$ of degree 1, the result of Eisenstein says that

$$
\prod_{r=1}^{p-1} \wp(\theta r / \pi)=1 / \pi^{2}
$$

Since

$$
\wp(\zeta z)=\zeta \wp(z), \quad \wp\left(\zeta^{2} z\right)=\zeta^{2} \wp(z) \quad \text { and } p=3 n+1
$$

then for $S$ any third set of residues $\bmod \pi$ we have:

$$
P_{S}=\prod_{x \in S} \wp(\theta x / \pi)
$$

which satisfies

$$
P_{S}^{3}=\prod_{x \in S} \wp(\theta x \pi) \wp(\theta \zeta x / \pi) \wp\left(\theta \zeta^{2} x / \pi\right)=1 / \pi^{2}
$$

The Cassels formula is as follows:

$$
\mathfrak{g}(1, \varepsilon, \pi)=-\frac{\varepsilon(\pi) p^{\frac{1}{3}} P_{S}}{\Omega(S, \varepsilon, \pi)}
$$

where $p^{\frac{1}{3}}$ is the real cubic root of $\mathrm{p}$ and $\pi \equiv 1 \bmod 3$.

$P_{S}$ gives a canonical way of extracting a cubic root of $\pi$, which, in turn, is necessary in the Stickelberger formula,

$$
\mathfrak{g}(1, \varepsilon, \pi)^{3}=-\pi^{2} \bar{\pi}=-p \pi
$$

If this would have not been the case, that $P_{S}$ exists, one would have had for a fixed $i ; 1 \leq i \leq 3$,

$$
\mathfrak{g}(1, \varepsilon, \pi)=\frac{\varepsilon\left(\zeta^{i_{0}}\right) p^{\frac{1}{3}} \varepsilon(\pi)^{\frac{1}{3}}}{\Omega(S, \varepsilon, \pi)}
$$

with $0 \leq \arg \left(\varepsilon(\pi)^{1 / 3}\right)<2 \pi / 3$

Remark We notice that we can rewrite this formula as in a very general shape. 


\section{A generalized of a formula of Cassels' type}

We shall investigate the possibility of a formula of Cassels'type for $n=5$; if such a formula exists, it could serve as a prototype for formulae for more general $n$.

Let $\varepsilon, \quad p, \quad \pi \mid p, \quad S$ and $\Omega(S, \varepsilon, \pi)$ be as before.

$$
P(1, \varepsilon, \pi)=-\frac{\mathfrak{g}(1, \varepsilon, \pi)}{\Omega(S, \varepsilon, \pi)} .
$$

We notice that $P(1, \varepsilon, \pi)$ lies in a much more bigger field than $K$.

Remark Let $K_{1}=K=\mathbb{Q}\left(\zeta_{n}\right)$ and $K_{2}=\mathbb{Q}\left(\zeta_{p}\right)$ the we denote $F$ the join of $K_{1}$ and $K_{2}$, i.e $F=K_{1} K_{2}$ then

$$
F / K_{2}=K_{1} K_{2} / K_{2} \equiv K_{1} / \mathbb{Q}
$$

so that $F / K_{2}$ is a Galois extension of $\mathbb{Q}$. Since $n$ and $p$ are relatively prime, the Galois group of $F / \mathbb{Q}$ is just the product of the underlying Galois groups i.e $\operatorname{Gal}(F / \mathbb{Q})=$ $\operatorname{Gal}\left(K_{1} / \mathbb{Q}\right) \times \operatorname{Gal}\left(K_{2} / \mathbb{Q}\right)$. The Galois group $\operatorname{Gal}(K / \mathbb{Q})$ acts on $P(1, \varepsilon, \pi)$ as follows

$$
P(1, \varepsilon, \pi)^{\sigma}=P\left(1, \varepsilon^{\sigma}, \pi\right)=P\left(1, \varepsilon, \pi^{\sigma}\right)
$$

for $\sigma \in \operatorname{Gal}(K / \mathbb{Q})$.

We have furthermore,

$$
\overline{P(1, \varepsilon, \pi)}=P\left(1, \varepsilon^{-1}, \pi\right) \text {. }
$$

Moreover $P(1, \varepsilon, \pi)$ satisfies

$$
P(1, \varepsilon, u \pi)=P(1, \varepsilon, \pi) .
$$

so that $P$ is invariant under the action of the unit group.

$$
P(1, \varepsilon, \pi)^{n}=-\mathfrak{g}(1, \varepsilon, \pi)^{n}=\omega(\varepsilon, \pi) .
$$

This know by the Stickelberger decomposition of Gauss sums. $\omega(\varepsilon, \pi)$ is a complex number and we can extract its roots. Then we can $\operatorname{fix} \arg \left(\omega(\varepsilon, \pi)^{1 / n}\right) \in$ $\left[-\pi / n, \pi / n\left[\right.\right.$. We then classify the pair of complex numbers $\left(P(1, \varepsilon, \pi), \omega(\varepsilon, \pi)^{1 / n}\right)$ according to their arclength. In fact they differ one from another by a complex $n^{\text {th }}$ root of unity. The numerical computation shows that

$$
\sum_{N(\pi) \leq 4.10^{4}} P(1, \varepsilon, \pi)=-149.738678-0.000079 i
$$

TABLE 1

\begin{tabular}{|c|c|c|c|c|c|}
\hline & sector 0 & sector 1 & sector 2 & sector 3 & sector 4 \\
\hline $\mathfrak{g}(1, \epsilon, \pi)$ & 744 & 848 & 868 & 868 & 848 \\
\hline$P(1, \varepsilon, \pi)$ & 862 & 882 & 775 & 775 & 882 \\
\hline Total & & & & & \\
\hline
\end{tabular}

there 4176 gauss sums, 744 in sector 0,848 in sector 1,868 in sector 2, 868 in sector3, 848 in sector 4 there 4176 corrected gauss sums , 862 in sector 0,881 in sector 1 , 776 in sector 2,776 in sector3, 881 in sector 4 


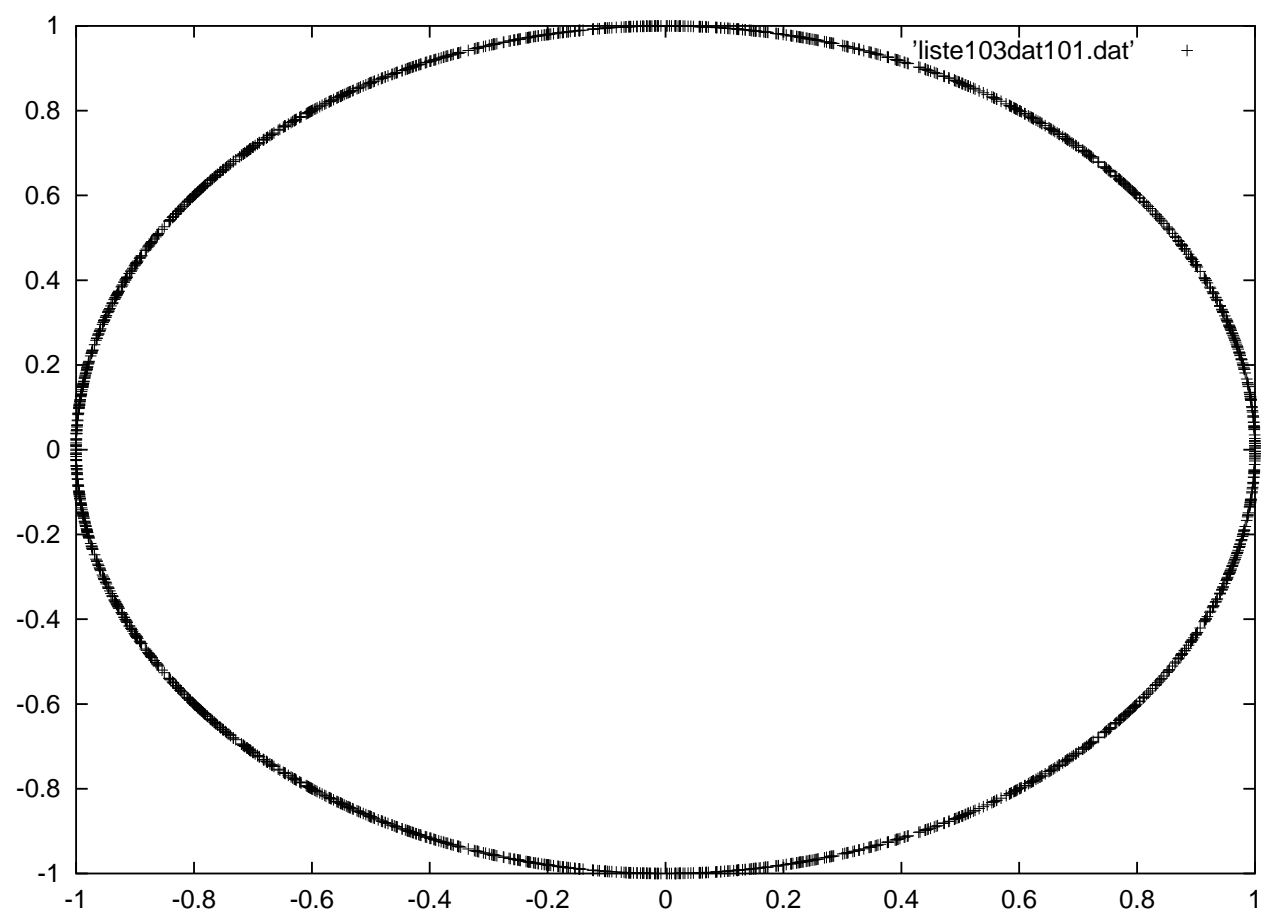

Figure 1. Graphic of the arguments of Gauss sums for $\pi \equiv 1$ $\left(\bmod \lambda^{3}\right) \quad N(\pi) \leq 20000$

Figure 1, figure 2 and figure 3 show the distribution of the arguments of Gauss sums. There are points which are very accurate than the other but this does not however says anything about the distribution. As has been proved by Patterson, this distribution is uniform. This graphs are consistent with the fact that the normalized Gauss sums are uniformly distributed on the unit circle, as was proved by Patterson. Note that there is no obvious bias as can be observed in the cubic case [SJP5] 


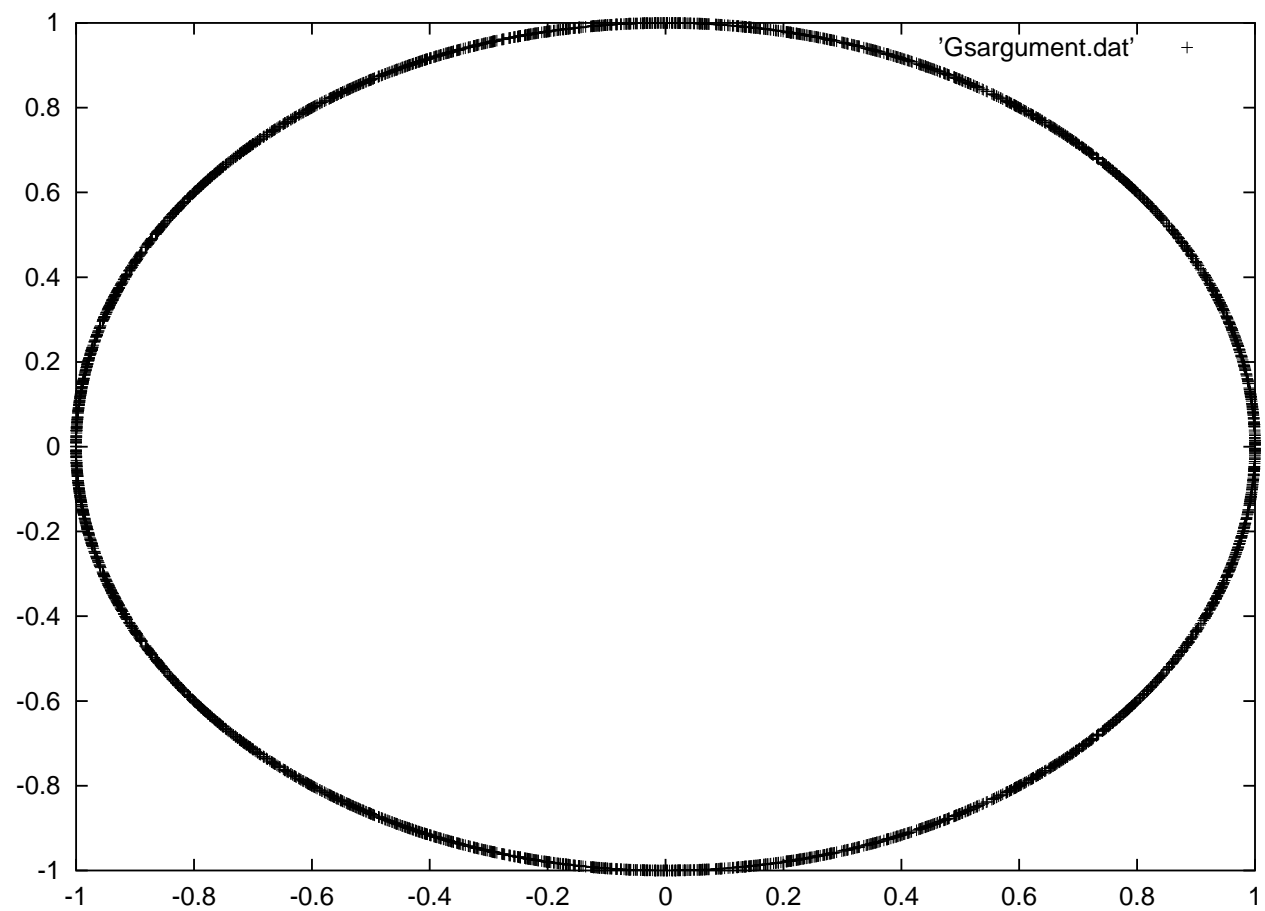

Figure 2. Graphic of the arguments of Gauss sums for $\pi \equiv 1$ $\left(\bmod \lambda^{3}\right) \quad N(\pi) \leq 40000$ 


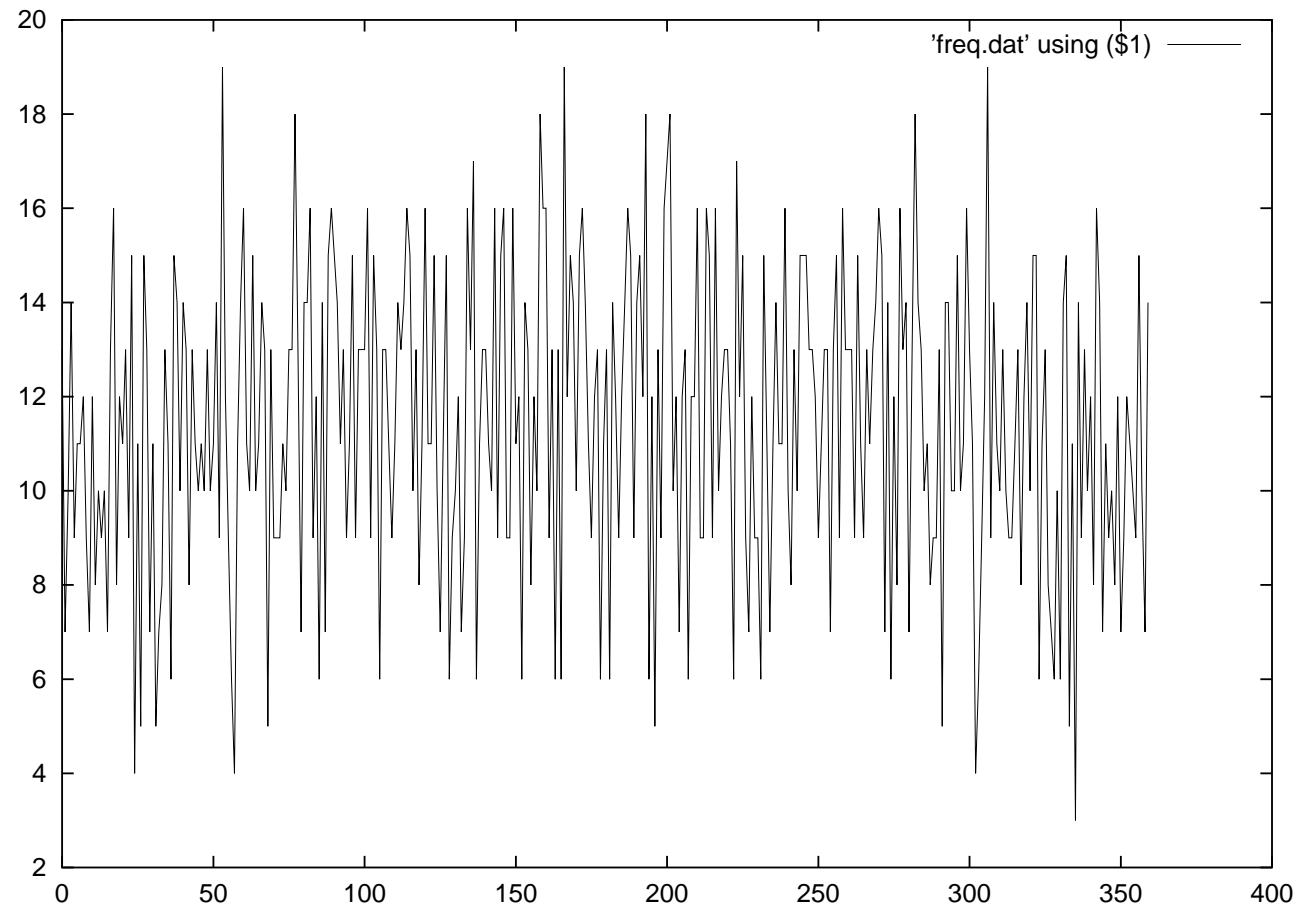

FiguRE 3. frequency in each 1 degree on the circle

This figure is the histogram of the frequency distribution of the argument of Gauss sums on intervals of arc-length one degree. 


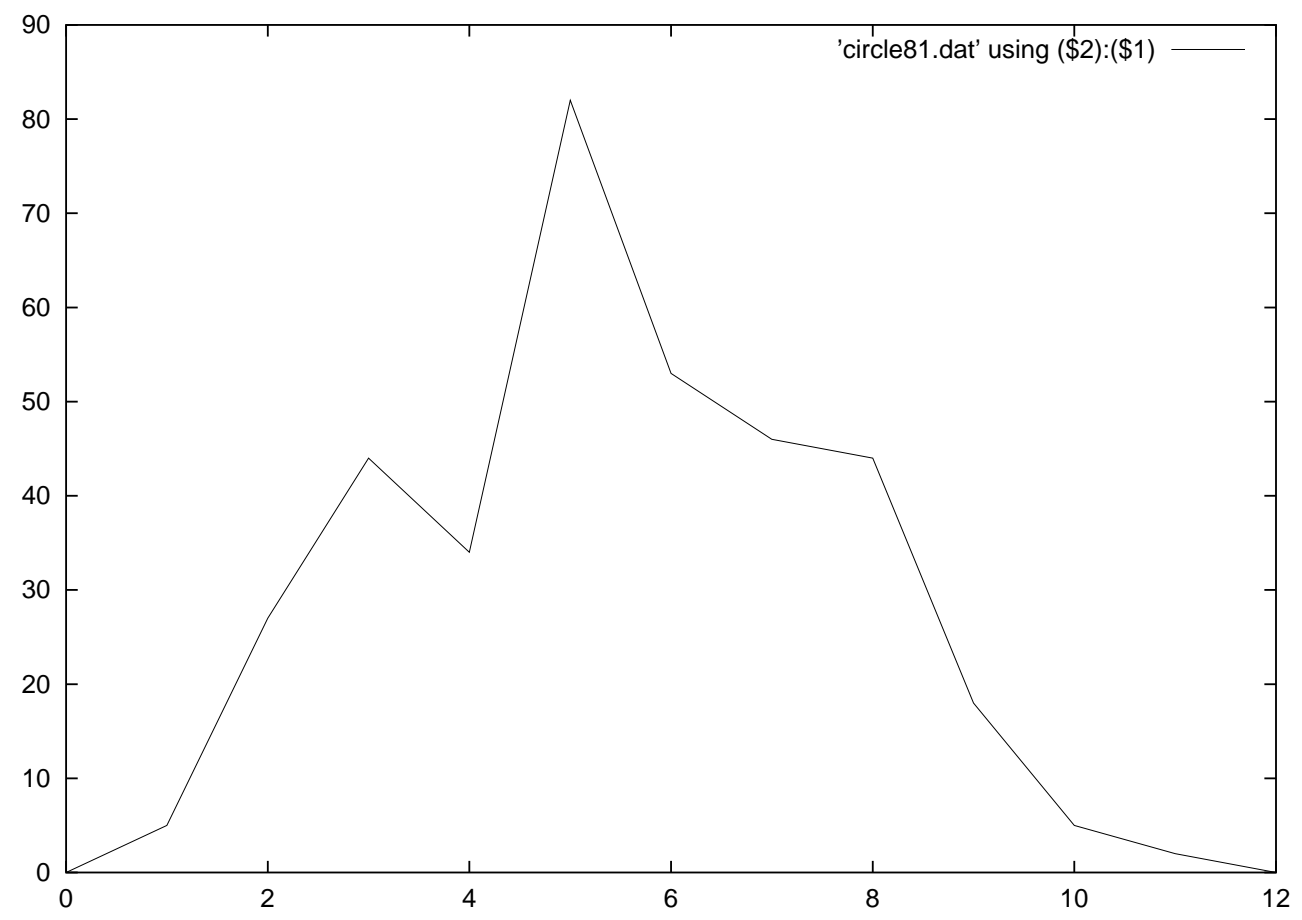

Figure 4. frequency in each 1 degree on the circle

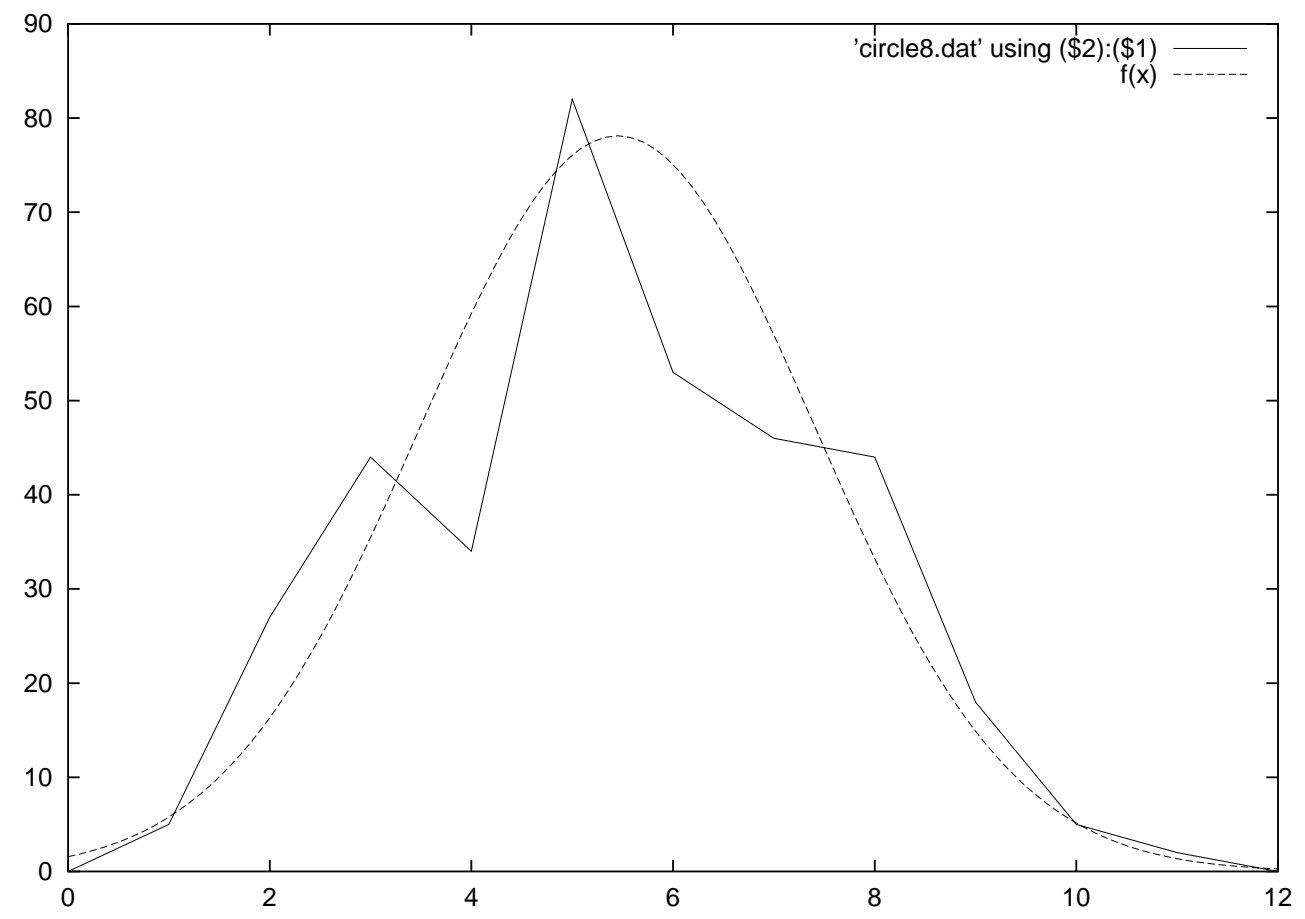

FIgURE 5. both graphs plotted one against another 
The plot of the graph shows some properties. The "tailing off" is observed. The graph of the frequencies shows that there is a symmetry on the distribution. We then classify, instead of the arguments of the Gauss sums, but the intervals of one degree arc length with respect to their frequencies. This has been done for different values of primes $p \equiv 1(\bmod 5), \quad p<X$. For example when

$$
X=2.10^{4}
$$

we understand this through the table as follows: in the first line we have the number of frequencies with which an interval of the form $\left[n^{0},(n+1)^{0}\right)$ appears and the second line gives number of the Gauss sums with argument in the corresponding range. from this table, we see that out of a sample of 1963 Gauss sums for the selected

TABLE 2

\begin{tabular}{|c|c|c|c|c|c|c|c|c|c|c|c|}
\hline different values of the frequency & 1 & 2 & 3 & 4 & 5 & 6 & 7 & 8 & 9 & 10 & 11 \\
\hline Number of sectors of 1 degree & 5 & 27 & 44 & 34 & 82 & 53 & 46 & 44 & 18 & 5 & 2 \\
\hline
\end{tabular}

value of $\pi \equiv 1 \bmod \lambda^{3}$, we notice that some sectors have a higher frequency rate while others just have a very few. From the computation, the sector $[249,250[$ first appears when we go over 20.000. In looking at the three first pictures, we preconize this fact by noticing that some points on the unit circle tend to be darker than others. This does not disprove the uniformly distribution of the arguments of Gauss sums proved by Patterson, it is, however,consistent with the uniform distribution proved by him. In this restricted range there is no sign of the same sort of biais as on sees in the cubic case. The statistical analysis makes such a bias unlikely. We have known it first from Kummer about this result. See comment about this fact from $[\mathbf{H P}]$. However, the shape of the plot of the above distribution is really meaningful. The bell shape shows a tendency that this distribution looks like a Gauss normal distribution $N(\mu, \sigma)$, where $\mu$ is the mean and $\sigma$ is the variance. Here the computed estimate values are

$$
\mu=5.45278 \quad \text { and } \quad \sigma=2.02
$$

Remark We could even find the mean $\mu$ without any computation. Since for primes $\pi \equiv 1(\bmod \lambda)^{3}$ there is at most $1,2,3$ or $4=\phi(5)$ different Gauss sums. This is a consequence of the decomposition of primes in cyclotomic fields. Then if $p \equiv 1(\bmod 5)$ then the Prime Number Theorem suggests that there is at most $c \frac{X}{\log X}$ primes of the sort. Some estimates of the constant $c$ says it is very close to 1 . Therefore

$$
\mu=\mu(X)=4 \cdot \frac{1}{360} \frac{X}{4 \log X}=\frac{1}{360} \frac{X}{\log X}
$$

so that for $X=20.000$ we obtain $\mu=5.61$ which may be considered to be closed to the estimated value 5.45278 . 


\section{Conjectures on the partial sums of the Gauss sums}

We are now interested in some partial summations. Some of this results are know from the elementary number theory. We use the computational result to support conjecture on partial sums. First we look at the following partial sums. We put them in pair so that we can discuss case after case. We first refresh our mind with partial summation Theorem.

Theorem 5.4. Let $f(n)$ and $g(n)$ be arithmetic functions. Consider the sum function

$$
F(x)=\sum_{n \leq x} f(n) .
$$

Let $a$ and $b$ be non negative integers with $a<b$. Then

$$
\sum_{n=a+1}^{b} f(n) g(n)=F(b) g(b)-F(a) g(a+1)-\sum_{n=a+1}^{b-1} F(n)(g(n+1)-g(n)) .
$$

Let $x$ and $y$ be non negative real numbers with $[y]<[x]$, and let $g(t)$ be a function with a continuous derivative on the interval $[y, x]$. Then

$$
\sum_{y<n \leq x} f(n) g(n)=F(x) g(x)-F(y) g(y)-\int_{y}^{x} F(t) g^{\prime}(t) d t .
$$

In particular, if $x \geq 2$ and $g(t)$ is continuously differentiable on $[1, x]$, then

$$
\sum_{n<x} f(n) g(n)=F(x) g(x)-\int_{1}^{x} F(t) g^{\prime}(t) d t .
$$

Proof. [Na] pp. 211.

From this theorem we deduce the following:

In the particular case of the statement, if we replace $g(n)$ by $\frac{1}{n}$, then

$$
\sum_{n \leq X} \frac{f(n)}{n}=\frac{F(X)}{X}+\int_{0}^{X} \frac{F(x)}{x^{2}} d x
$$

and we furthermore obtain

$$
\begin{aligned}
\frac{1}{X} \int_{0}^{X} F(y) d y & =\frac{1}{X} \sum_{n \leq X} f(n) \int_{n}^{X} d y \\
& =\frac{1}{X} \sum_{n \leq X} f(n)(X-n) \\
& =\sum_{n \leq X} f(n)\left(1-\frac{n}{X}\right)
\end{aligned}
$$

Let $\mu(n)$ be the Möbiüs function,

$$
\mu(n)=\left\{\begin{array}{ccc} 
& 1 & \text { if } n=1 \\
(-1)^{k} & \text { if } & n \text { is the product of } \mathrm{k} \text { distinct primes } \\
& 0 & \text { if } \mathrm{n} \text { is divisible by a square }
\end{array}\right.
$$


We define the functions:

$$
F(X)=\sum_{n \leq X} \mu(n) n^{\frac{1}{2}}
$$

we contrast

$$
F(X)=\sum_{n \leq X} \mu(n) n^{\frac{1}{2}} \quad \text { and } \quad H(X)=\sum_{\substack{N(\pi) \leq X \\ \pi \equiv 1 \bmod \lambda^{3}}} \mathfrak{g}(1, \varepsilon, \pi) \log (N(\pi)) .
$$

Here the reason for which we decide this form is as follows. The Gauss sum has the modulus $|\mathfrak{g}(1, \varepsilon, \pi)|=\sqrt{N(\pi)}$ and for the powers of $\pi$ the Gauss sums vanish, therefore it is better to use the Von Mangoldt $\Lambda$ function.

In fact if we put

$$
M(X)=\sum_{n \leq X} \mu(n)
$$

then $M(X)$ is a function which is on average $O(\sqrt{X})$ therefore

$$
\sum_{n \leq X} \mu(n) n^{\frac{1}{2}}=O(X)
$$

In contrary $\sqrt{n}$ can be replaced by any other arithmetic function, then we cannot easily determine the estimate. In replacing $\sqrt{n}$ by the Gauss sum, we are consistent with this conjecture.

\subsection{Conjecture I.}

$$
H(X)=\sum_{\substack{N(\pi) \leq X \\ \pi \equiv 1 \bmod \lambda^{3}}} \mathfrak{g}(1, \varepsilon, \pi) \log (N(\pi))=c \cdot X^{6 / 5}+O\left(X^{1+\epsilon}\right)
$$

Where $c$ is a constant and $\varepsilon$ is a positive real number.

Note that $H(X)$ is a real function. Because at the primes, which are primary $\pi^{*} \equiv 1$ $(\bmod \lambda)^{3}$ there is at most one Gauss sum $\mathfrak{g}\left(1, \varepsilon, \pi^{*}\right)$, because any other primary associate prime differs from the previous one by a fifth power of a unit. This is not necessary the case for other primes. Moreover the inverse of a Galois element yields complex conjugate on the Gauss sums, so that in each congruent class of primes, the Gauss sums appear in simultaneously pair of complex conjugates and therefore the function $H(X)$ is real. At each prime factor $\pi \mid p$, if $\mathfrak{g}\left(1, \varepsilon, \pi^{*}\right)$ is not real the sum

$$
\mathfrak{g}\left(1, \varepsilon, \pi^{*}\right)+\mathfrak{g}\left(1, \varepsilon^{-1}, \pi^{*}\right)
$$

is either positive or negative.

We would expect this sign appears totally at random so that it would behave like the Möbius function. For this reason the function $H(X)$ would cross the x-axis infinitely many times as $X$ tends to $\infty$. This is however a surprising result that let us to conjecture. 
6.2. Conjecture II. The function $H(X)$ has the following property: there exists a positive real $\mathrm{A}$, such that for all $X>A, H(X)>0$.

All of them are derived from the theorem 5.4. We will consider successively functions

$$
f_{1}(p)=\sum_{\substack{p \equiv 1 \\ \pi \equiv, \quad(\bmod 5)}} \mathfrak{g}(1, \varepsilon, \pi) \log (N(\pi))
$$

and

Since

$$
f_{2}(p)=f_{1}(p) \times \frac{1}{p}
$$

$$
\left.H(X)=\sum_{\substack{N(\pi) \leq X \\ \pi \equiv 1}} \mathfrak{g}(1, \varepsilon, \pi) \log \lambda\right)^{3}(N(\pi))
$$

then

$$
H_{1}(X)=\frac{1}{X} \int_{1}^{X} H(Y) d Y=\sum_{N(\pi)=p \leq X} f_{1}(p)\left(1-\frac{N(\pi)}{X}\right)
$$

and for

$$
K(X)=\sum_{\substack{N(\pi) \leq X \\ \pi \equiv 1}} \frac{\mathfrak{g}(1, \varepsilon, \pi) \log (N(\pi))}{N(\pi)}
$$

we have

$$
K_{1}(X)=\frac{1}{X} \int_{1}^{X} K(Y) d Y=\sum_{N(\pi)=p \leq X} f_{2}(p)\left(1-\frac{N(\pi)}{X}\right)
$$

so that we obtain if we assume the conjecture I, that:

$$
H_{1}(X)=\frac{1}{X} \int_{1}^{X} H(Y) d Y=c \cdot \frac{5}{11} X^{\frac{6}{5}}+O\left(X^{1+\epsilon}\right)
$$

from theorem 5.4,

$$
K(X)=\frac{H(X)}{X}+\int_{1}^{X} \frac{H(Y)}{Y^{2}} d Y=c \cdot X^{1 / 5}+O\left(X^{-1+\epsilon}\right)
$$

and then it follows that

$$
K_{1}(X)=\frac{1}{X} \int_{1}^{X} K(Y) d Y=c \cdot \frac{5}{6} X^{\frac{1}{5}}+O\left(X^{-1+\epsilon}\right)
$$

The same observation is valid for

$$
\tilde{H}(X)=\sum_{\substack{N(\pi) \leq X \\ \pi \equiv 1}} \frac{\mathfrak{g}(1, \varepsilon, \pi) \log (N(\pi))}{\Omega\left(S_{0}, \varepsilon, \pi\right)}
$$

and

$$
\tilde{K}(X)=\sum_{\substack{N(\pi) \leq X \\ \pi \equiv 1}} \frac{\mathfrak{g}(1, \varepsilon, \pi) \log (N(\pi))}{\Omega\left(S_{0}, \varepsilon, \pi\right) N(\pi)}
$$


In the next pages, we explain here a sample of different graphs we have described. 


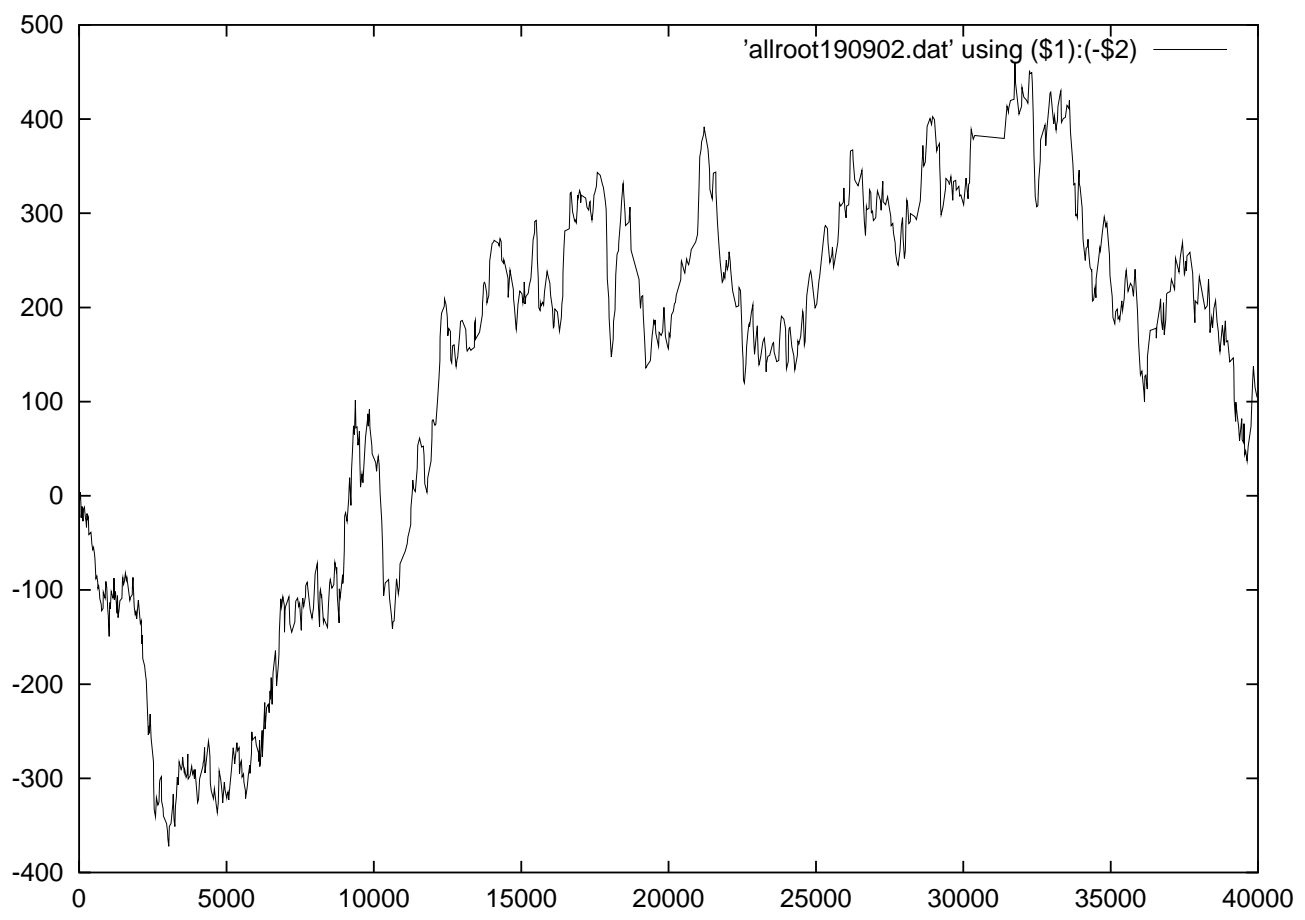

FiguRE 6. $g(X)=\sum_{\substack{n \leq X \\ 0<X<40.000}} \Omega\left(S_{0}, \varepsilon, \pi\right) \log (N(\pi))$

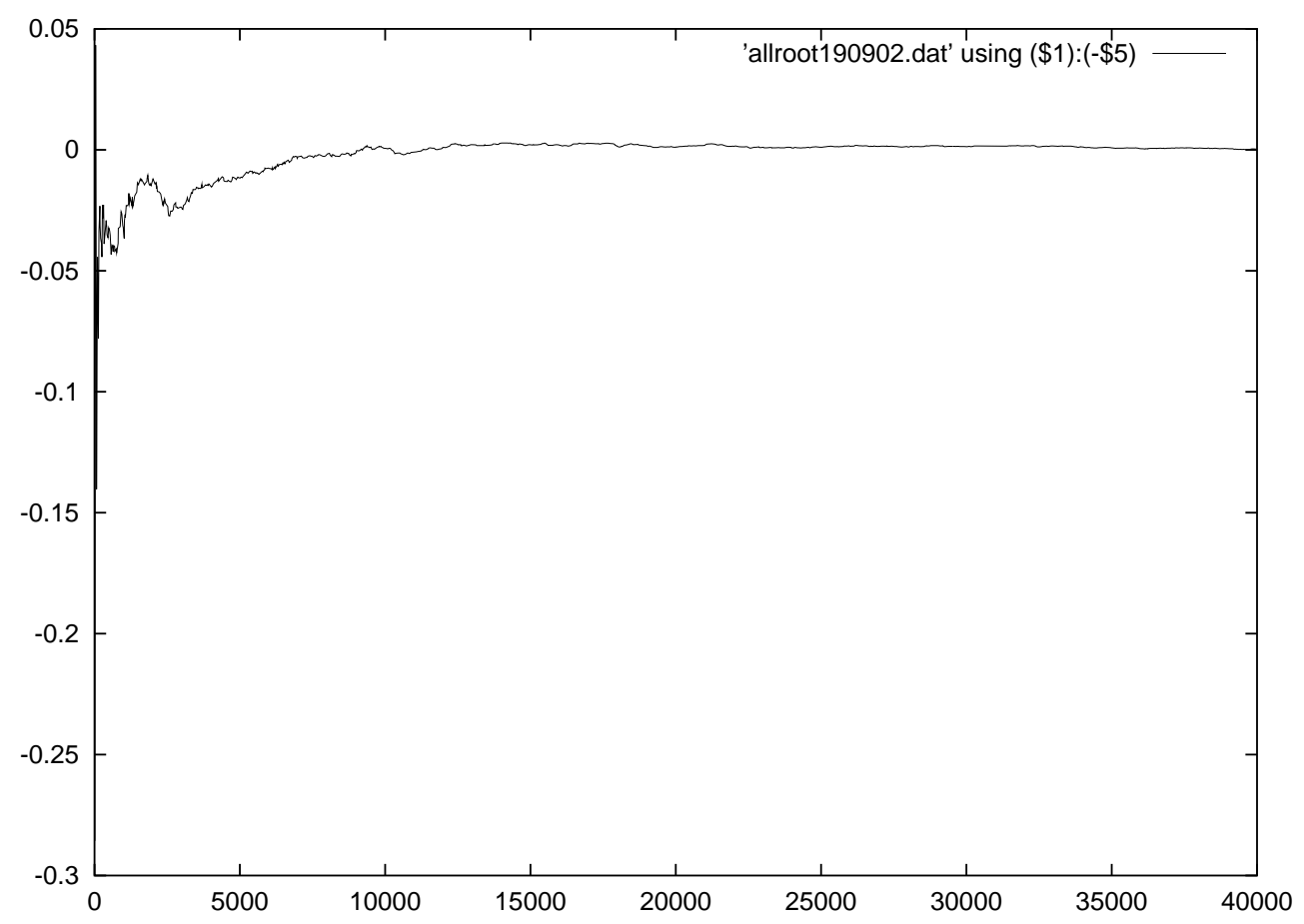

FiguRE 7. $g(X)=\frac{1}{X^{6 / 5}} \sum_{\substack{n \leq X \\ 0<X<0.000}} \Omega\left(S_{0}, \varepsilon, \pi\right) \log (N(\pi))$ 


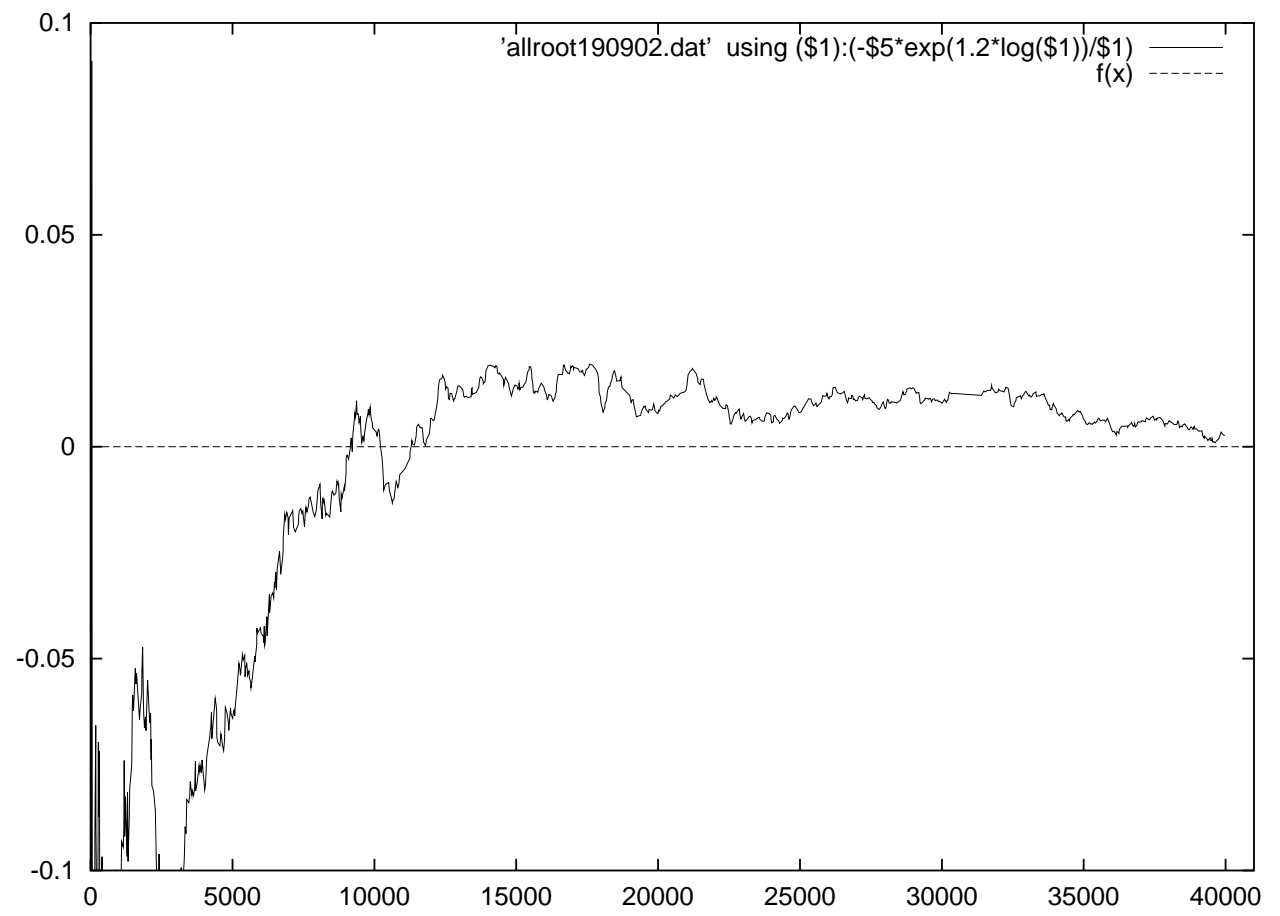

FiguRE 8. $g(X)=\frac{1}{X} \sum_{\substack{0<X<X \\ 0<40.000}} \Omega\left(S_{0}, \varepsilon, \pi\right) \log (N(\pi))$

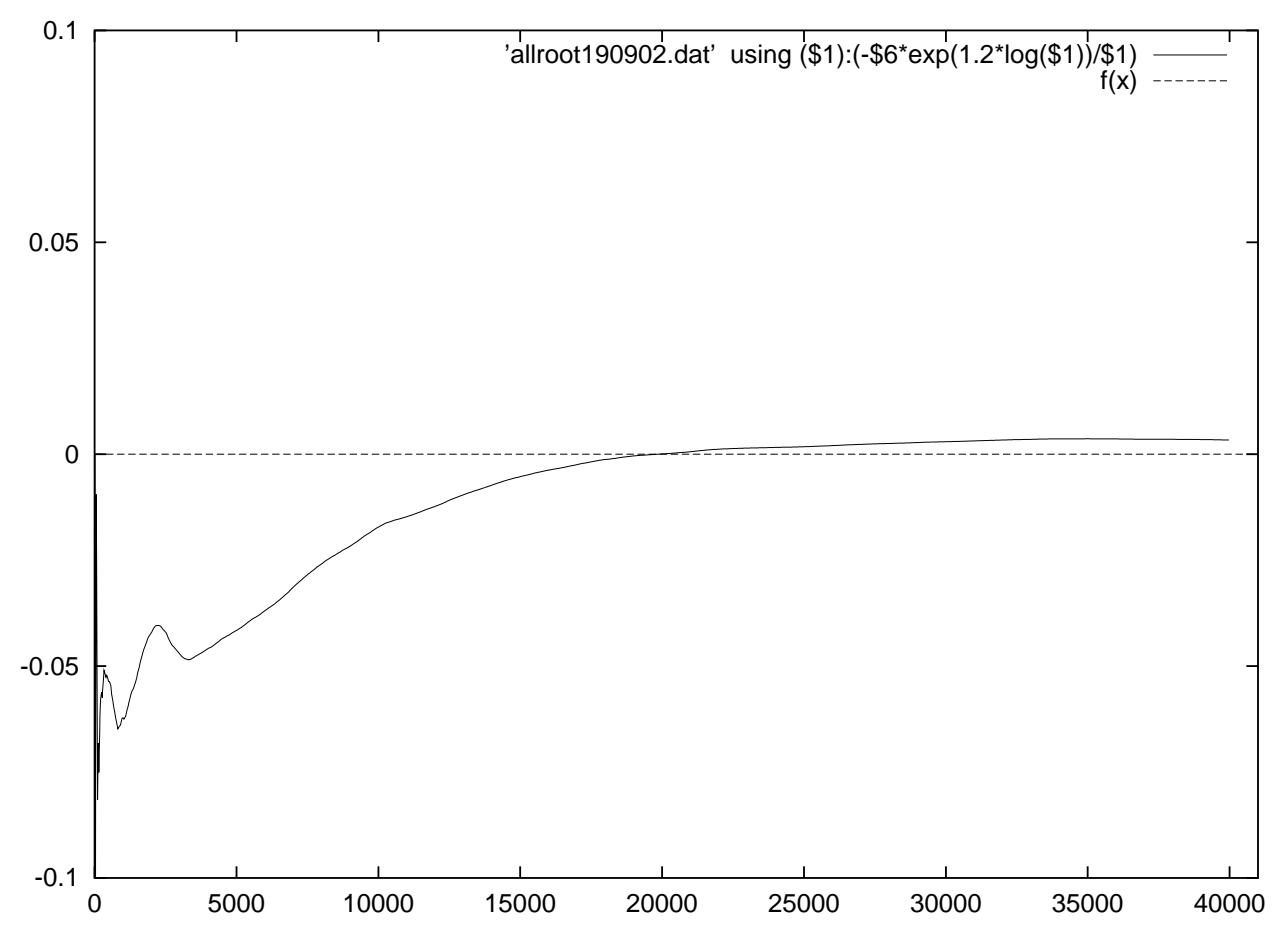

FiguRE 9. $g(X)=\frac{1}{X} \sum_{\substack{n \leq X<X 0.000 \\ 0<x}} \Omega\left(S_{0}, \varepsilon, \pi\right) \log (N(\pi))\left(1-\frac{N(\pi)}{X}\right)$ 


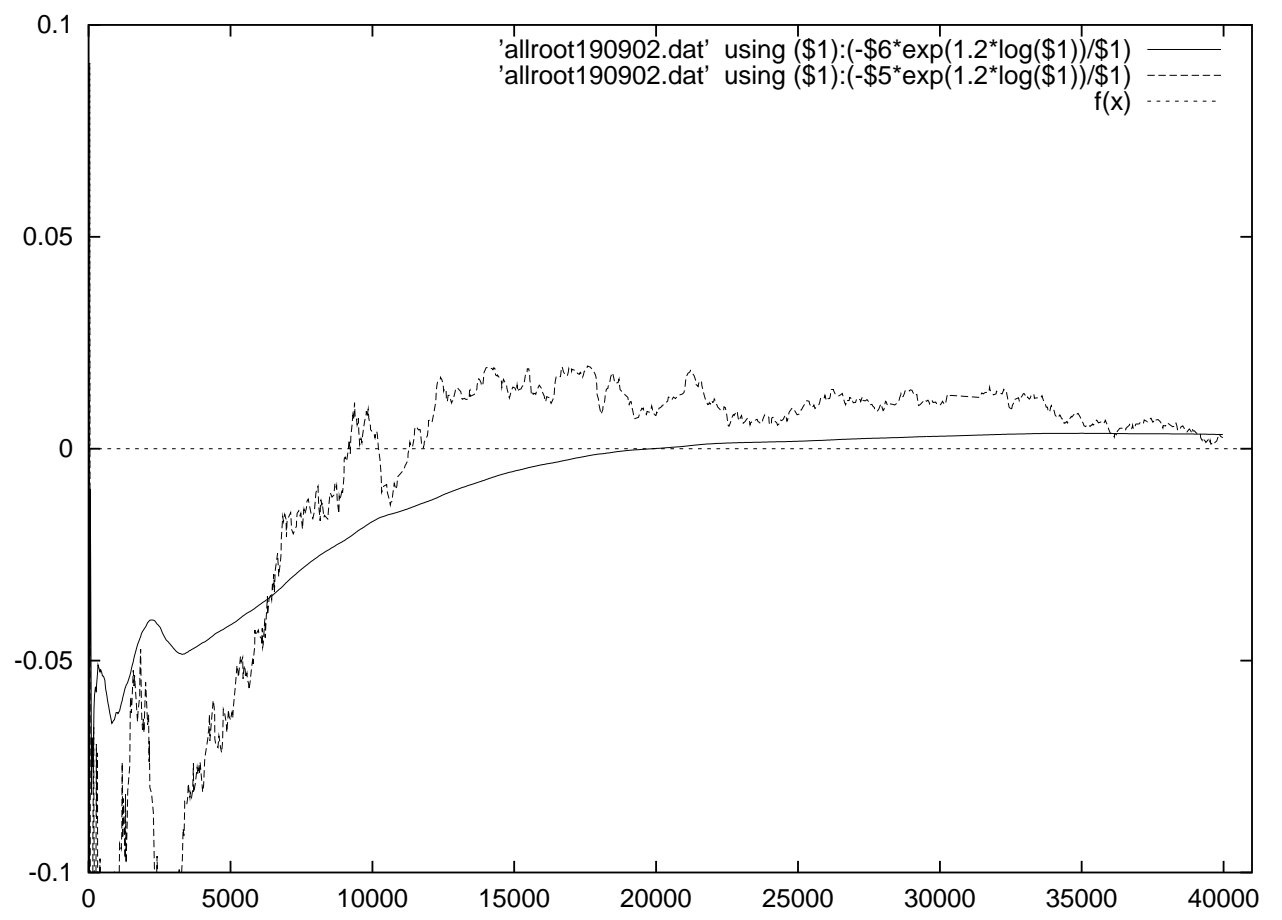

FiguRE 10. $g(X)=\quad \frac{1}{X} \sum_{\substack{n \leq X \\ 0<X<0.000}} \Omega\left(S_{0}, \varepsilon, \pi\right) \log (N(\pi))$ and $\quad f(X)=\frac{1}{X} \sum_{\substack{n \leq X \\ 0<X<40.000}} \Omega\left(S_{0}, \varepsilon, \pi\right) \log (N(\pi))\left(1-\frac{N(\pi)}{X}\right)$

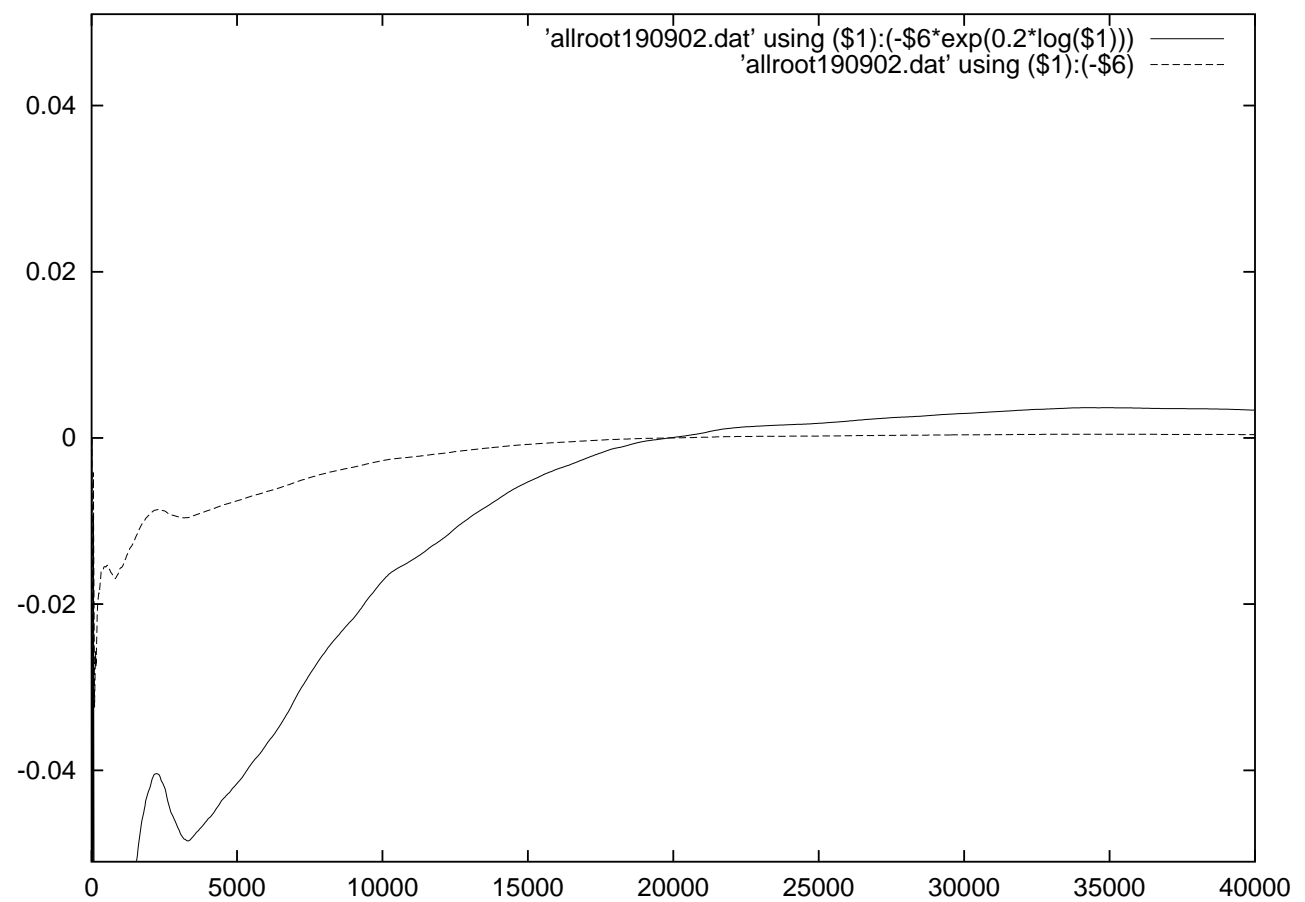

Figure 11. $g(X)=\frac{1}{X} \sum_{\substack{n \leq X \\ 0<X<40.000}} \Omega\left(S_{0}, \varepsilon, \pi\right) \log (N(\pi))\left(1-\frac{N(\pi)}{X}\right)$ and $\sum_{\substack{n \leq X \\ 0<X<40.000}} \Omega\left(S_{0}, \varepsilon, \pi\right) \log (N(\pi))\left(1-\frac{N(\pi)}{X}\right)$ 
We observe that if $\frac{1}{X} \sum_{\substack{n \leq X \\ 0<X<40.000}} \Omega\left(S_{0}, \varepsilon, \pi\right) \log (N(\pi))$ would behave like $X$ or $X^{6 / 5}$ then the constant would be very small if it is not 0 . 


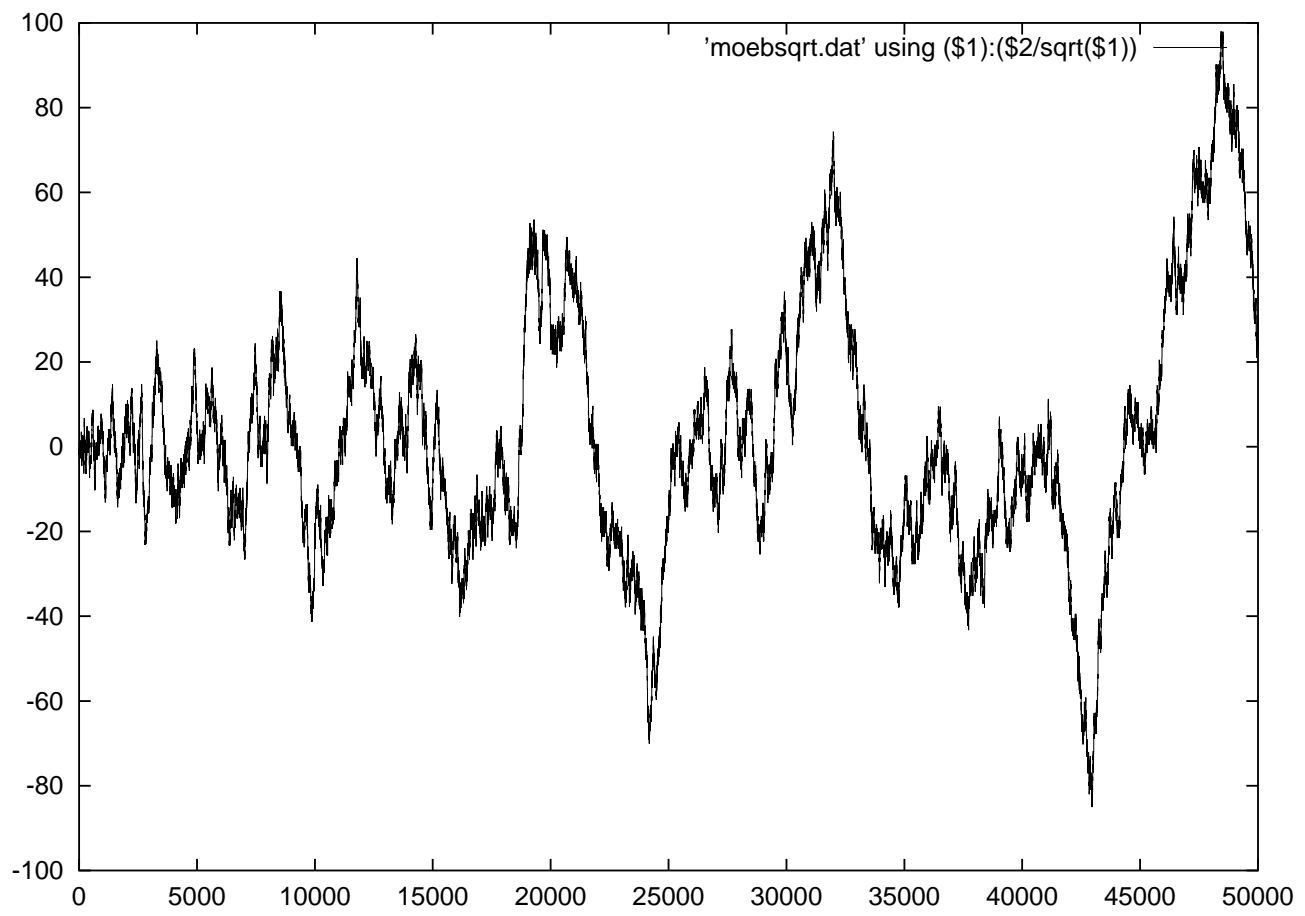

FiguRE 12. $f(X)=X^{-1 / 2} \sum_{\substack{n \leq X, 0<X<50.000}} \mu(n) n^{1 / 2}$

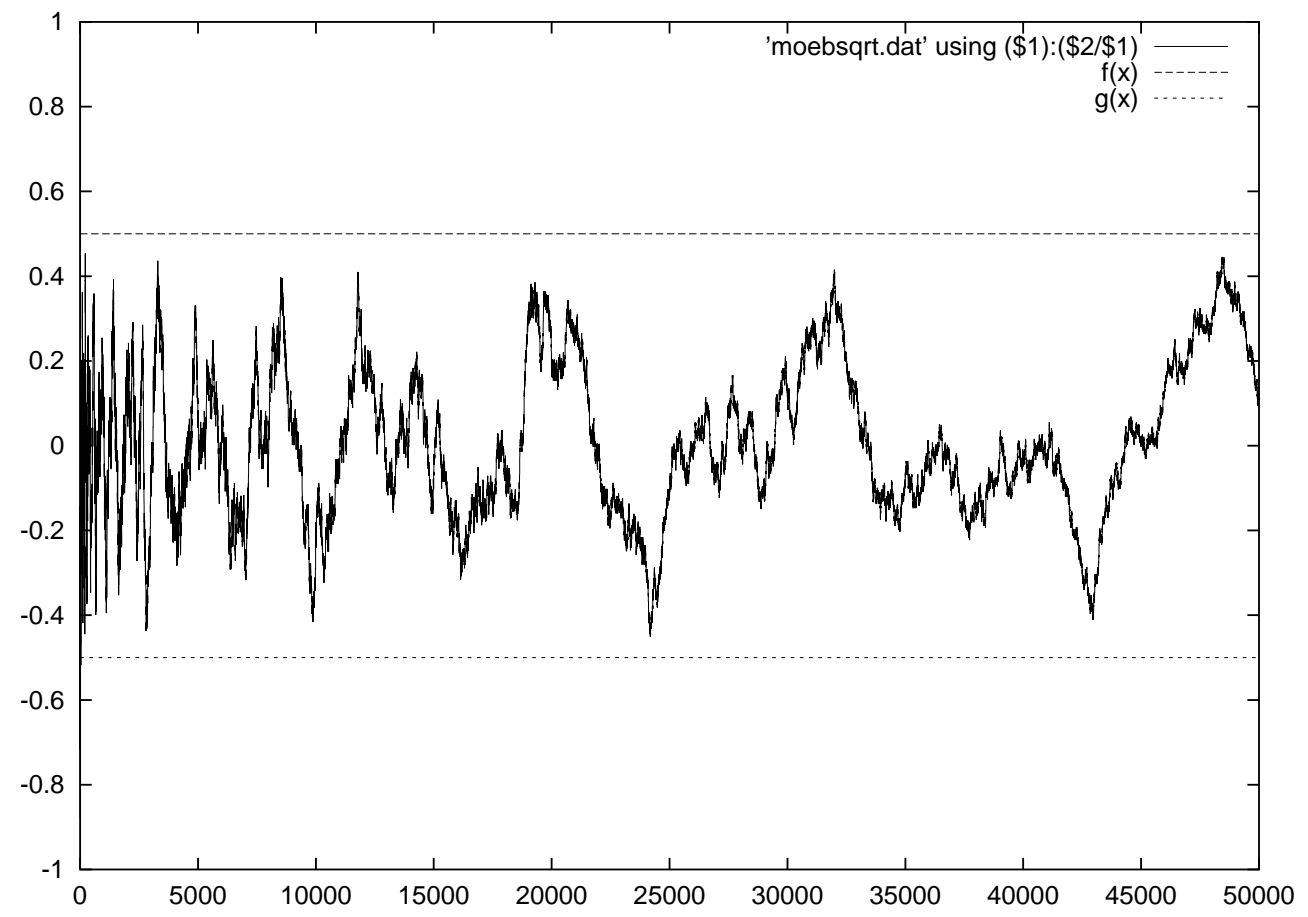

FiguRE 13. $f(X)=\frac{1}{X} \sum_{\substack{n \leq X<5, \cdot 000 \\ 0<X}} \mu(n) n^{1 / 2}$ 
This in fact suggests that the constant term would be 0.5 . It is known that

$$
\sum_{n<X} \mu(n)=O\left(X^{1 / 2}\right)
$$

so that

$$
\sum_{n<X} \mu(n) \sqrt{n}=O(X) \quad \text { i.e } \quad \frac{1}{X} \sum_{n<X} \mu(n) \sqrt{n}=O(1)
$$

The Mertens conjecture suggested that the absolute values of the constant would be at most 1, but it has been disprove by A. M. Odlyzko and H. J. J. te Riele, where they showed that for $X>\exp \left(3.21 .10^{64}\right)$, the constant is unfortunately greater that 1. 


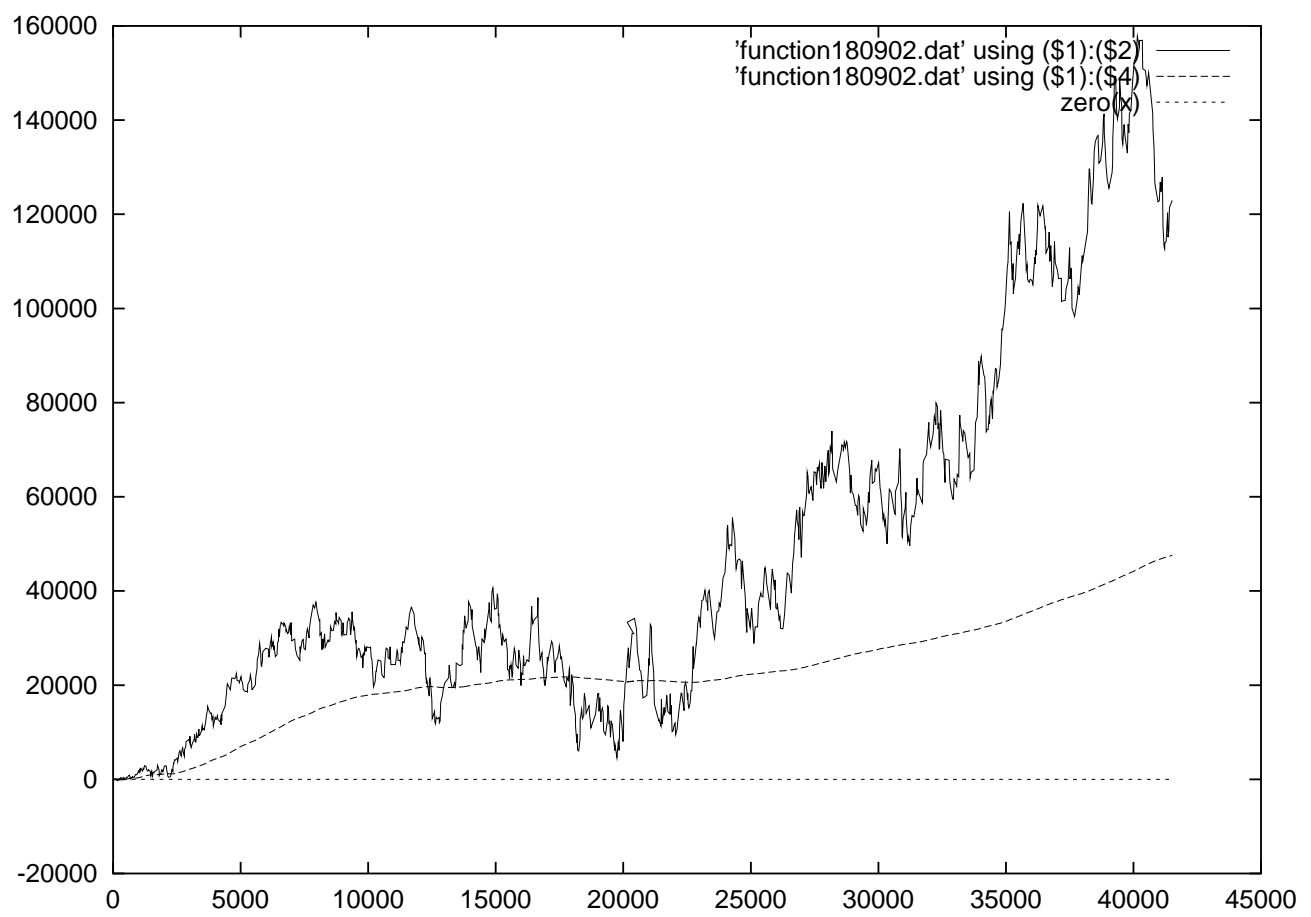

FigURE 14. $\sum_{\substack{\pi \equiv 1\left(\bmod \lambda^{3}\right), N(\pi) \leq X, X<40000}} \mathfrak{g}(1, \varepsilon, \pi) \log (N(\pi))$

$$
\text { and } \sum_{\substack{\pi \equiv 1\left(\bmod \lambda^{3}\right), N(\pi) \leq X, X \leq 40000}} \mathfrak{g}(1, \varepsilon, \pi) \log (N(\pi))\left(1-\frac{N(\pi)}{X}\right)
$$




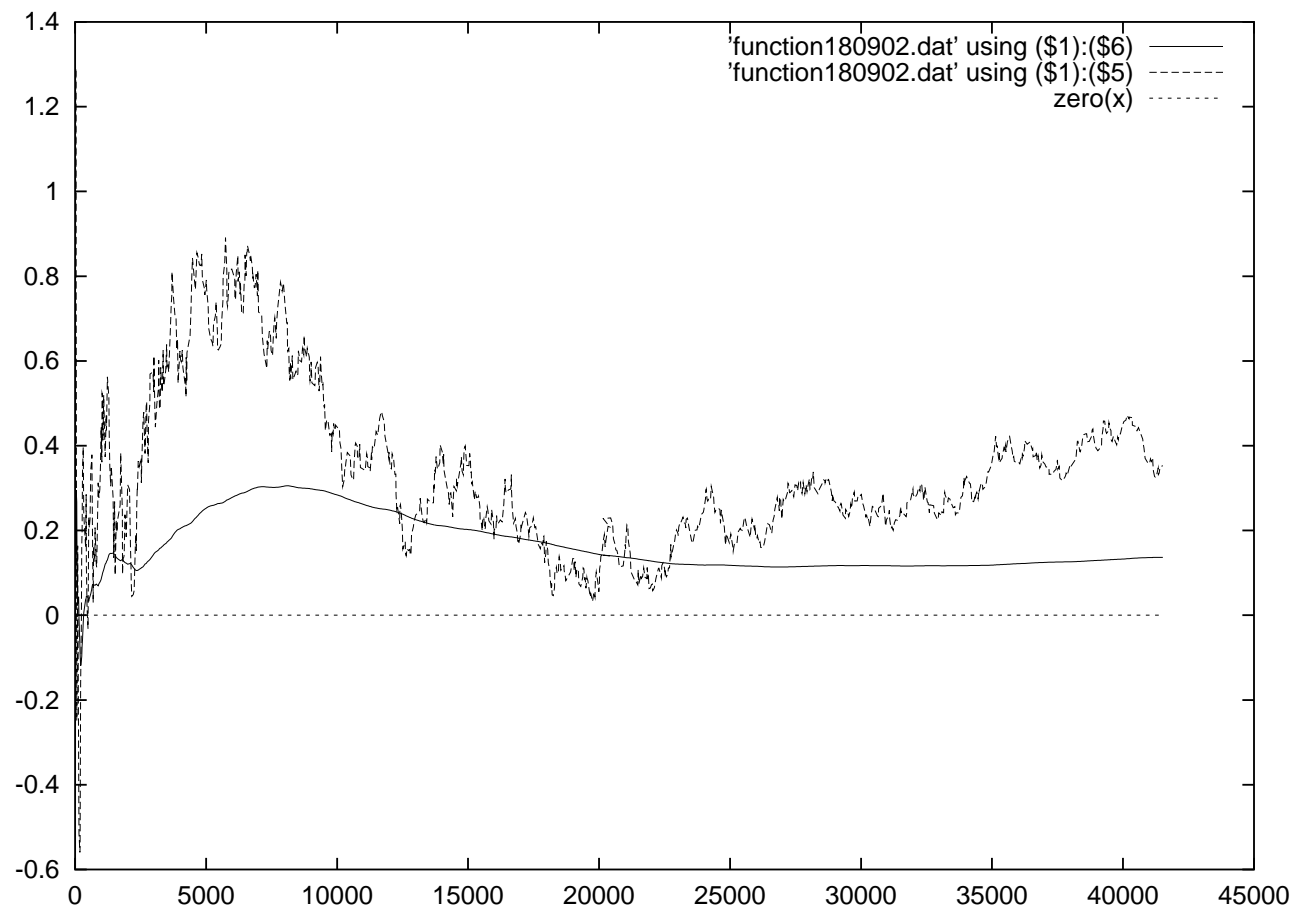

FiguRE 15. $\frac{1}{X^{6 / 5}} \sum_{\substack{\pi \equiv 1\left(\bmod \lambda^{3}\right), N(\pi) \leq X, X \leq 40000}} \mathfrak{g}(1, \varepsilon, \pi) \log (N(\pi))$ and $X^{-\frac{6}{5}} \sum_{\substack{\pi \equiv 1(\bmod \lambda 3), N(\pi) \leq X, X \leq 40000}} \mathfrak{g}(1, \varepsilon, \pi) \log (N(\pi))\left(1-\frac{N(\pi)}{X}\right)$

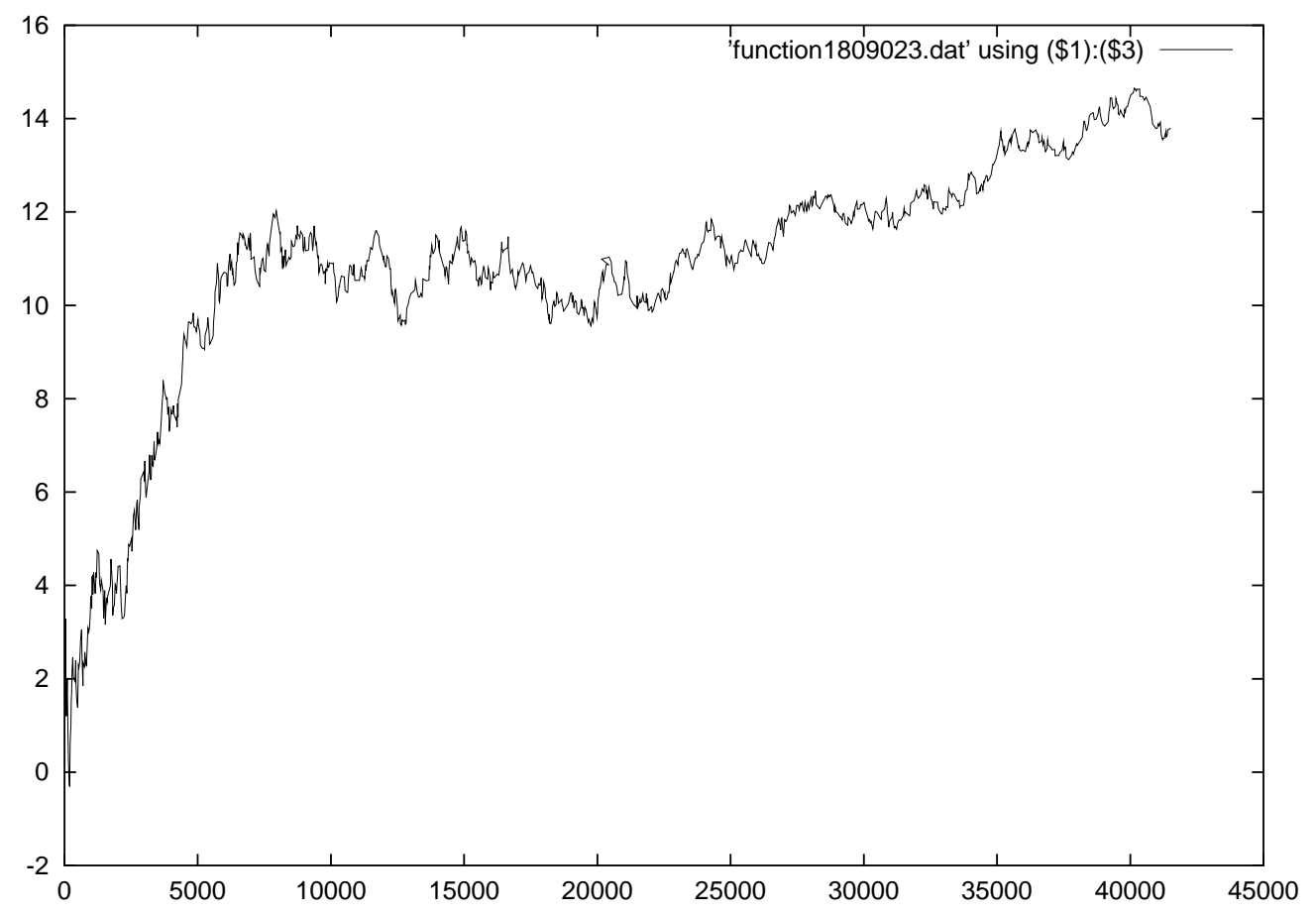

FIGURE 16. $\sum_{\substack{\pi \equiv 1\left(\bmod \lambda^{3}\right), N(\pi) \leq X, X \leq 40000}} \frac{\mathfrak{g}(1, \varepsilon, \pi) \log (N(\pi))}{N(\pi)}$ 


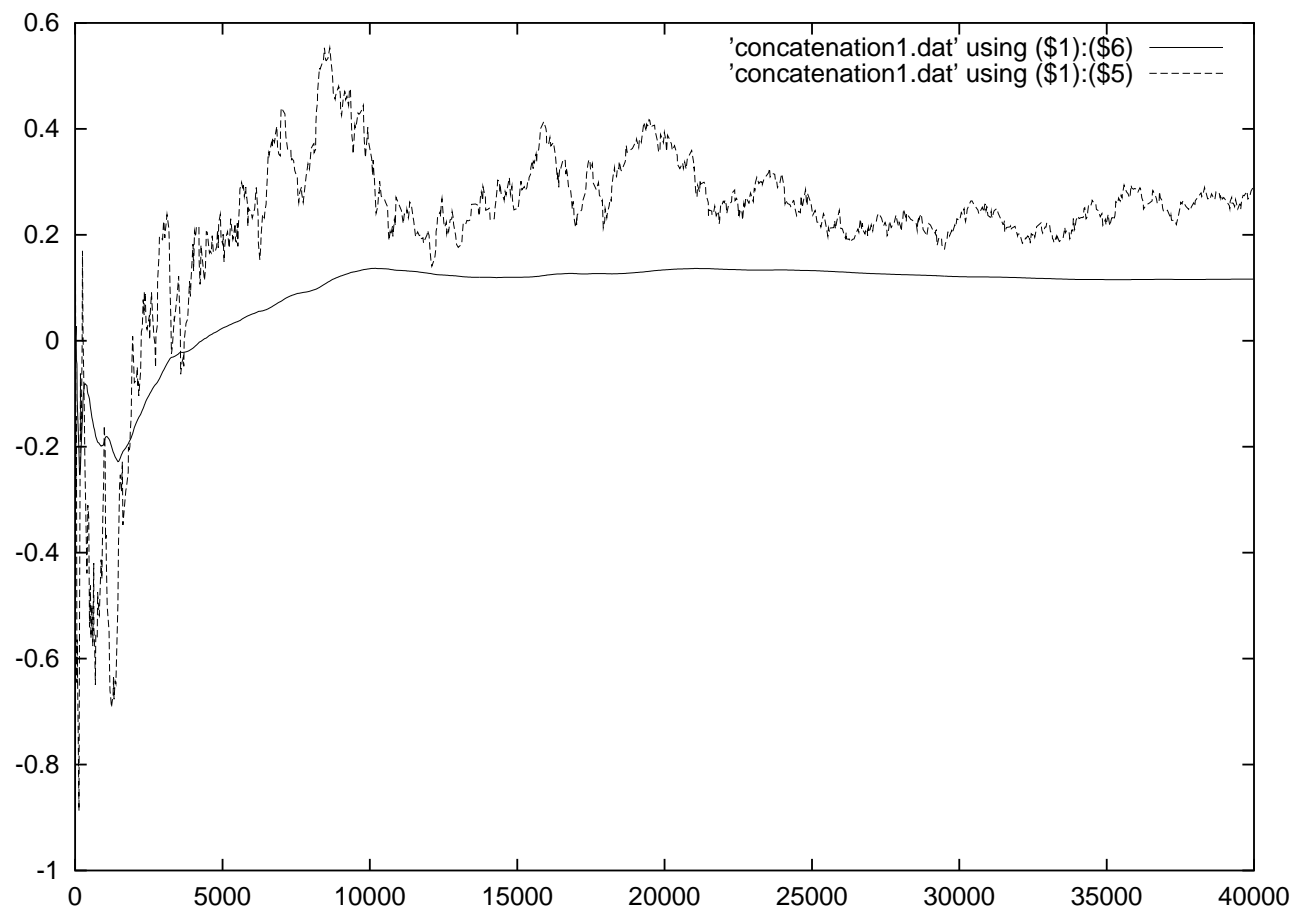

FIGURE 17.

$$
\begin{gathered}
f(X)=X^{-\frac{6}{5}} \sum_{\substack{\left.\pi \equiv 1<\bmod \lambda^{3}\right), N(\pi) \leq X, X \leq 40000}} \frac{\mathfrak{g}(1, \varepsilon, \pi) \log (N(\pi))}{\Omega\left(S_{0}, \varepsilon, \pi\right)} \\
\text { and } \quad X^{-\frac{6}{5}} \quad \sum_{\substack{\left.\pi \equiv 1 \\
\text { Nad } \lambda^{3}\right), N(\pi) \leq X, X \leq 40000}} \frac{\mathfrak{g}(1, \varepsilon, \pi) \log (N(\pi))}{\Omega\left(S_{0}, \varepsilon, \pi\right)}\left(1-\frac{N(\pi)}{X}\right)
\end{gathered}
$$




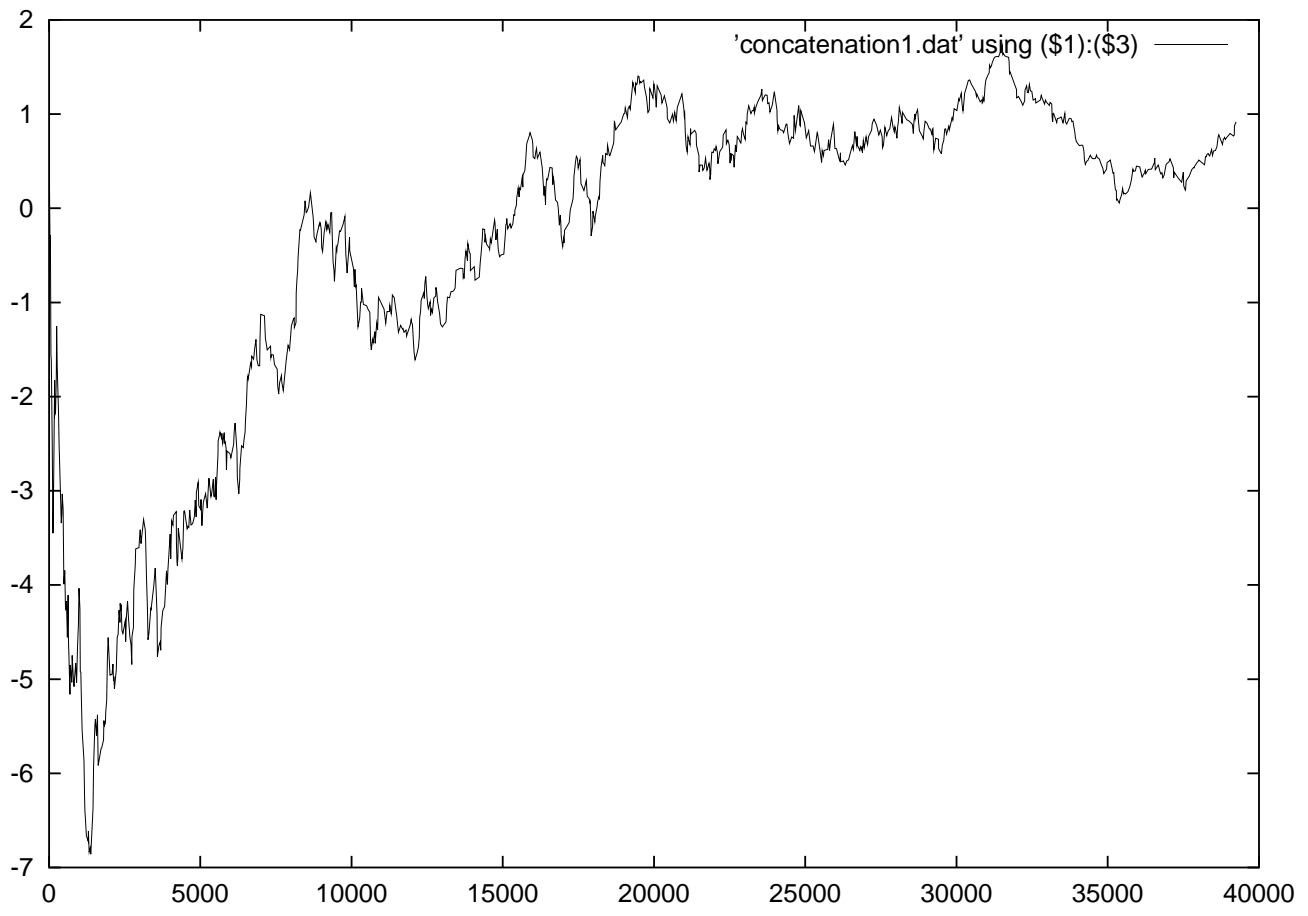

FIGURE 18. $\sum_{\substack{\pi \equiv 1\left(\bmod \lambda^{3}\right), N(\pi) \leq X, X \leq 40000}} \frac{\mathfrak{g}(1, \varepsilon, \pi) \log (N(\pi))}{\Omega\left(S_{0}, \varepsilon, \pi\right) N(\pi)}$

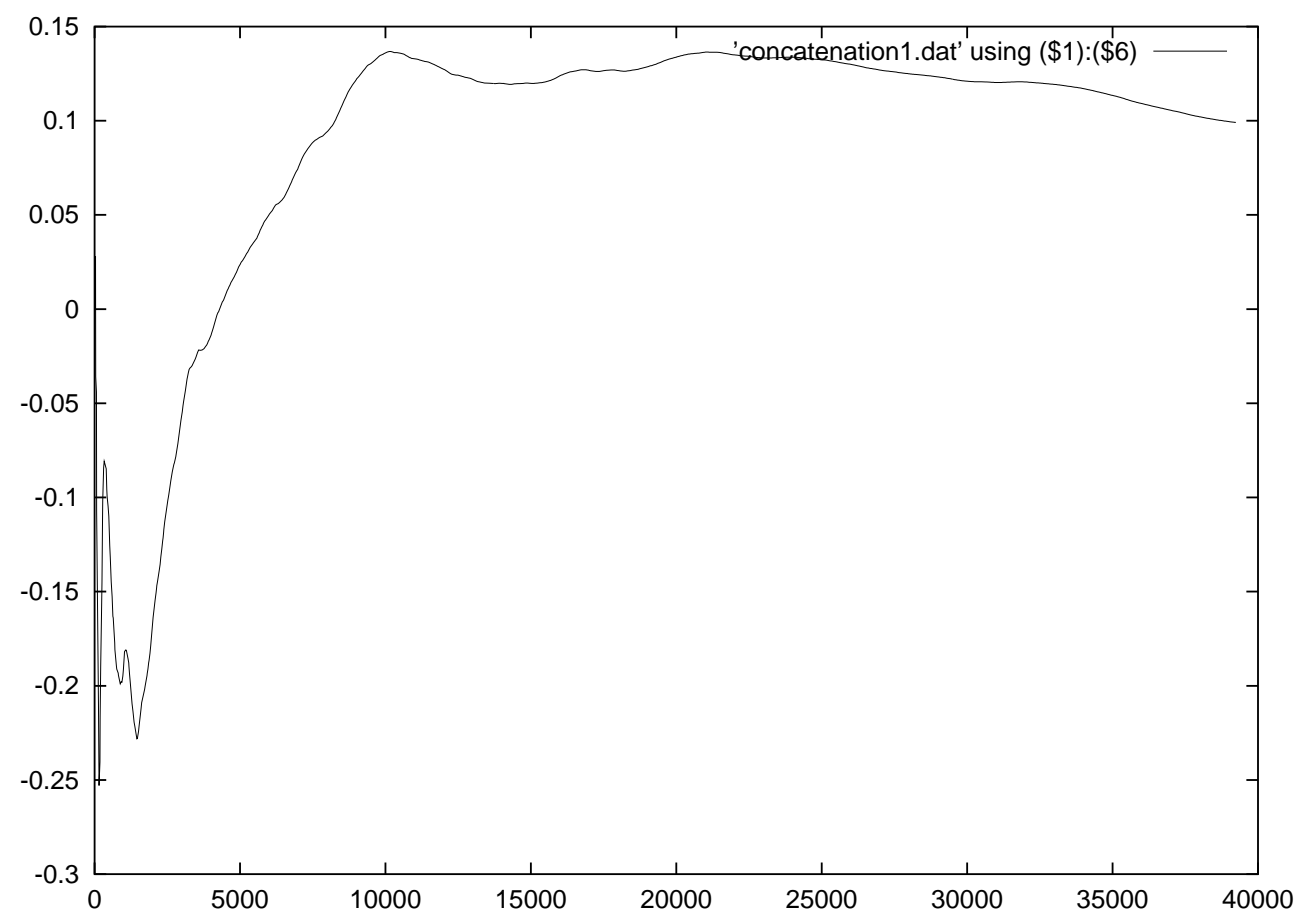

Figure 19.

$$
X^{-\frac{6}{5}} \sum_{\substack{\pi \equiv 1 \\ N(\pi) \leq X, X \leq 40000}} \frac{\mathfrak{g}(1, \varepsilon, \pi) \log (N(\pi))}{N(\pi)} \text { and }
$$


6.3. Conclusion. The phenomenon observed near the origin is very common to such a question.

All the pictures drawn with the Gauss sums are different from the pictures drawn with the Möbiüs function. We expected to see all of them behave the same way. This is far from our expectation. The partial sumation involving Gaus sums have a tendency to have a constant sign for large values of $\mathrm{X}$. This means that the sign of Gauss sums is far from being totally random.

We also notice that the integral version of any of the functions involving Gauss sums, is very smooth.

The quotient of Gauss sums multiply by a suitable root of unity, which would represent a generalization of the determination of the sign of the quadratic Gauss sums, does not have effect on the functions we plotted. Nevertheless, they have a very much elegant asymptotic behaviour that one would expect.

We have numerically confirmed a conjecture of Patterson within the range of computation.

In the earlier chapter, we simply come to the conclusion that the test for the validity of a possible Cassels-McGettrick formula, that would represent a generalization of the determination of the sign of the quadratic Gauss sums, does not hold. The statistical evidence indicates that even with a broad interpretation of the formulation of such a formula, no such formula exists.

Since the choice of the root of unity is done with respect to some geometry, one may think of modifying the fundamental region as to succeed. This is not done here and this would be hard to undertake surgery beyond the 3-dimensional space. Our result is also a warning, that one would need a different approach in the theory of Gauss sums. Patterson introduced his inaugural lecture at Goettingen in 1981, with a title "Gaussschen Summe: ein Thema mit Variationen" and 20 years later in another talk on the recent development in this theory, came to the conclusion that one may need more means and strategies than that one actually has. 



\section{CHAPTER 6}

\section{APPENDIX}

\section{The Gauss periods or the Lagrange resolvents}

This section has nothing to do with the discussion about the origin of Gauss periods or Lagrange resolvents. If the latter appears earlier than the Gauss sums, the contemporary scientists extended the research on problems raised up by Gauss and may have named these sums after Gauss. However any computation of Gauss sum induces an implicit dermination of the Lagrange resolvents. However, Hilbert used the expression "Würzelzahl " to refer to the term used by Lagrange in his famous Rflexions sur la rsolution algbrique des quations.

In this step we are not taking part to this discussion, but we will emphasize on a particular case, which is more or less less obvious. In general the Lagrange resolvents are algebraic integers in a certain field $K / \mathbb{Q}$. Here we prove that upon certain conditions, all the Lagrange resolvents are rational integers such that the corresponding Gauss sum has a very simple shape. We give a simple example to explain this fact.

Let $p \geq 3$ and $q \neq p$ be prime numbers and $\zeta_{p}, \zeta_{q}$ are respectively a $p^{\text {th }}$ and $q^{\text {th }}$ primitive root of 1 . Let $n \geq 2$ be the order of $q \bmod p$ such that

$$
p(q-1) \mid\left(q^{n}-1\right) \quad \text { and } n \mid p-1 .
$$

Put $f=\left(q^{n}-1\right) / p$ and $e=(p-1) / n$. In the field $K=\mathbb{Q}\left(\zeta_{p}\right)$, any finite prime ideal $\mathfrak{q}$ above $q$ yields a finite residue class field $\mathbb{F}=\mathbb{Z}\left[\zeta_{p}\right] / \mathfrak{q}$, and $\mathbb{F} \cong \mathbb{F}_{q^{n}}$.

Definition 6.1. Let $\alpha \in \mathbb{Z}\left[\zeta_{p}\right]$ be a generator of $\mathbb{F}^{\times}$such that $\alpha^{f} \equiv \zeta_{p}(\bmod \mathfrak{q})$. The $p$ elements

$$
\eta_{i}=\sum_{j=0}^{f-1} \zeta_{q}^{\operatorname{Tr}_{K / \mathbb{Q}}\left(\alpha^{i+p j}\right)}, \quad 0 \leq i \leq p-1
$$

are called Gauss periods, and the Gauss sum is nothing else but

$$
G=\sum_{i=0}^{q^{n}-2} \zeta_{p}^{i} \zeta_{q}^{\operatorname{Tr}_{K / \mathbb{Q}}\left(\alpha^{i}\right)}=\sum_{i=0}^{p-1} \eta_{i} \zeta_{p}^{i}
$$

Remark This form of the Gauss sums is the computable version. One has to find a method to compute the $\eta_{i}$ and then use its properties to perform a good algorithm. See $[\mathbf{F . T 1}],[\mathbf{F . T 2}],[\mathbf{G . M}],[\mathbf{H}]$.

We can extend the index of $\eta_{i}$ to $\mathbb{Z}$ as follows: define

$$
\eta_{i+k p}:=\eta_{i}
$$


Let $g$ be a primitive root modulo $q$ such that

$$
g \equiv \alpha^{\frac{q^{n}-1}{q-1}} \bmod \mathfrak{q}
$$

and let $\tau$ be the automorphism of $\mathbb{Q}\left(\zeta_{q}\right) / \mathbb{Q}$ such that $\tau\left(\zeta_{q}\right)=\zeta_{q}^{g}$.

$$
\tau\left(\eta_{i}\right)=\sum_{j=0}^{f-1} \zeta_{q}^{g \operatorname{Tr}_{K / \mathbb{Q}}\left(\alpha^{i+p j}\right)}=\sum_{j=0}^{f-1} \zeta_{q}^{\operatorname{Tr}_{K / \mathbb{Q}}\left(g \alpha^{i+p j}\right)}=\sum_{j=0}^{f-1} \zeta_{q}^{\operatorname{Tr}_{K / \mathbb{Q}}\left(\alpha^{\frac{p\left(q^{n}-1\right)}{p(q-1)}+i+p j}\right)}=\eta_{i}
$$

for each $i$. Since $\tau$ generated $\operatorname{Gal}\left(\mathbb{Q}\left(\zeta_{q}\right) / \mathbb{Q}\right)$, this proves that:

Lemma 6.1. Let $p \geq 3$ and $q \neq p$ be prime numbers such that there exits $n \geq 2$ factor of $p-1$ and $\left.p(q-1) \mid q^{n}-1\right)$. Let $\eta_{i}$ defined as before, then for

$$
0 \leq i \leq p-1 \quad \eta_{i} \in \mathbb{Z}
$$

and

$$
\eta_{q i}=\eta_{i}
$$

Proof. We have already proved that $\tau\left(\eta_{i}\right)=\eta_{i}$ for all $\tau \in \operatorname{Gal}\left(\mathbb{Q}\left(\zeta_{q}\right) / \mathbb{Q}\right)$ so that $\eta_{i}$ is invariant under $\operatorname{Gal}\left(\mathbb{Q}\left(\zeta_{q}\right) / \mathbb{Q}\right)$.

$$
\eta_{q i}=\sum_{j=0}^{f-1} \zeta_{q}^{\operatorname{Tr}_{K / \mathbb{Q}}\left(\alpha^{q i+p j}\right)}=\sum_{j=0}^{f-1} \zeta_{q}^{\operatorname{Tr}_{K / \mathbb{Q}}\left(\alpha^{i+p j}\right)}, \quad 0 \leq i \leq p-1
$$

We define the polynomial in one indeterminate

$$
G(X)=\sum_{i=0}^{q^{n}-2} X^{i} \zeta_{q}^{\operatorname{Tr}_{K / \mathbb{Q}}\left(\alpha^{i}\right)}
$$

then

$$
G(X) \equiv \sum_{i=0}^{p-1} \eta_{i} X^{i} \bmod \left(X^{p}-1\right)
$$

so that $G=G\left(\zeta_{p}\right)$ and a direct computation shows that

$$
G(1)=\sum_{i=0}^{p-1} \eta_{i}=-1 \quad \text { and } \quad G\left(\zeta_{p}^{i}\right) G\left(\zeta_{p}^{-i}\right)=q^{n}, \quad 1 \leq i \leq p-1
$$

$G(1)=-1$ has generated discussion about the real definition of Gauss sums. Thus in [w1] we see that one can correct the sign by introducing the Möbiüs function.

If $n$ is even, then from $\eta_{q i}=\eta_{i}$ we deduce that $\eta_{-i}=\eta_{q^{\frac{n}{2}} i}=\eta_{i}$ so that $G\left(\zeta_{p}^{i}\right)=$ $G\left(\zeta_{p}^{-i}\right)$, thus $G= \pm q^{n / 2}$. Then working modulo $\left(\zeta_{p}-1\right)$, we obtain

$$
G=q^{\frac{n}{2}}
$$


If $n$ is odd, then $e=(p-1) / n$ is even. We then define the Gaussian periods

$$
\theta_{i}=\sum_{l=0}^{n-1} \zeta_{p}^{g^{i+e l}}, \quad 0 \leq i \leq e-1 .
$$

This results in the well-known lemma

Lemma 6.2. $\left\{\theta_{0}, \theta_{1} \cdots \theta_{e-1}\right\}$ forms a normal integral basis of $\mathbb{Q}\left(\theta_{0}\right) / \mathbb{Q}$, the subfield of $\mathbb{Q}\left(\zeta_{p}\right)$ of degree e.

We also notice that

$$
\theta_{g^{i+e}}=\theta_{i} \text { for } i \geq 0 .
$$

this observation is a consequence of the fact that $q^{n} \equiv g^{p-1} \equiv 1 \bmod p$ implies that $g^{e} \equiv q^{t} \bmod p$ for some $t$ prime to $n$. We have therefore

$$
G\left(\zeta_{p}\right)=\theta_{0}+\sum_{i=0}^{p-2} \theta_{g^{i}} \zeta_{p}^{g^{i}}=\theta_{0}+\sum_{i=0}^{e-1} \theta_{g^{i}} \sum_{j=0}^{n-1} \zeta_{p}^{g^{i+e j}}
$$

i.e

$$
G\left(\zeta_{p}\right)=\theta_{0}+\sum_{i=0}^{e-1} \theta_{g^{i}} \theta_{i}
$$

The multiplication among the $\theta_{i}$ yields the following facts. Since $\left(\theta_{i}\right)_{(0 \leq i \leq e-1)}$ form a basis there is matrix A with coefficients $a_{(i, j)}$ such that

$$
\theta_{0} \theta_{i}=\sum_{j=0}^{e-1} a_{i, j} \theta_{j}
$$

for $0 \leq i, j \leq e-1$. Since $\theta_{i+k e}=\theta_{i}$ we see that $a_{(i+k e, j+l e)}=a_{(i, j)}$, however, one notices that if $\sigma \in \operatorname{Gal}\left(\mathbb{Q}\left(\zeta_{p}\right) / \mathbb{Q}\right)$ is such that $\sigma\left(\zeta_{p}\right)=\zeta_{p}^{g}$ then $\sigma\left(\theta_{0} \theta_{i}\right)=\theta_{1} \theta_{i+1}$. Therefore by taking the conjugates of $\theta_{0} \theta_{i}$ we have

$$
\theta_{i} \theta_{j}=\sum_{k=0}^{e-1} a_{(j-i, k-i)} \theta_{k}=\sum_{k=0}^{e-1} a_{(i-j, k-j)} \theta_{k}=\theta_{j} \theta_{i}
$$

This proves that

Finally we have

$$
a_{(i, j)}=a_{(-i, j-i)}
$$

$$
\theta_{i} \theta_{j}=A\left(\begin{array}{c}
\theta_{j} \\
\theta_{j+1} \\
\cdots \\
\cdots \\
\cdots \\
\theta_{j+e-1}
\end{array}\right)=\theta_{j}\left(\begin{array}{c}
\theta_{j} \\
\theta_{j+1} \\
\cdots \\
\cdots \\
\cdots \\
\theta_{j+e-1}
\end{array}\right) .
$$

We have therefore proved that the gauss periods $\theta_{0}, \cdots, \theta_{e-1}$ are exactly the eigen values of the matrix $A$, and $\operatorname{det}(X I-A)$ is the minimal polynomial of the periods. Thus $\mathbb{Q}\left(\theta_{0}\right) \cong \mathbb{Q}(A)$ by $\theta_{0} \longmapsto A$. 


\section{Example}

We take $p=3$ and $q=5$, then $K=\mathbb{Q}\left(\zeta_{3}\right)$. The order of 5 modulo 3 is 2 , so that 5 does not split in $\mathbb{Z}\left[\zeta_{3}\right]$. The residue class field at 5 is

$$
\mathbb{Z}\left[\zeta_{3}\right] /(5)=\left\{a+b \zeta_{3} ; \quad 0 \leq a, b \leq 5\right\} .
$$

and the element $\alpha=1+3 \zeta_{3}$ is a generator. Furthermore $f=8$ and $e=1$.

$$
\begin{aligned}
& \eta_{0}=\sum_{j=0}^{7} \zeta_{q}^{\operatorname{Tr}_{K / \mathbb{Q}}\left(\alpha^{0+3 j}\right)}=\zeta_{5}^{2}+\zeta_{5}^{0}+\zeta_{5}^{4}+\zeta_{5}^{0}+\zeta_{5}^{3}+\zeta_{5}^{0}+\zeta_{5}+\zeta_{5}^{0}=3 \\
& \eta_{1}=\sum_{j=0}^{7} \zeta_{q}^{\operatorname{Tr}_{K / \mathbb{Q}}\left(\alpha^{1+3 j}\right)}=\zeta_{5}^{4}+\zeta_{5}^{0}+\zeta_{5}^{3}+\zeta_{5}^{2}+\zeta_{5}+\zeta_{5}^{4}+\zeta_{5}^{2}+\zeta_{5}^{3}=-2 \\
& \eta_{2}=\sum_{j=0}^{7} \zeta_{q}^{\operatorname{Tr}_{K / \mathbb{Q}}\left(\alpha^{2+3 j}\right)}=\zeta_{5}^{2}+\zeta_{5}^{4}+\zeta_{5}^{4}+\zeta_{5}^{3}+\zeta_{5}^{3}+\zeta_{5}+\zeta_{5}+\zeta_{5}^{2}=
\end{aligned}
$$

One verifies that:

$$
\begin{gathered}
\eta_{0}+\eta_{1}+\eta_{2}=-1 \\
\eta_{2}=\eta_{-1}=\eta_{1} . \\
G\left(\zeta_{3}\right)=\eta_{0}+\zeta_{3} \eta_{1}+\zeta_{3}^{2} \eta_{2}=\eta_{0}+\eta_{1}\left(\zeta_{3}+\zeta_{3}^{2}\right)=3+(-2)(-1)=5=5^{\frac{2}{2}} \\
G\left(\zeta_{3}^{-1}\right)=\eta_{0}+\zeta_{3} \eta_{2}+\zeta_{3}^{2} \eta_{1}=\eta_{0}+\eta_{1}\left(\zeta_{3}+\zeta_{3}^{2}\right)=3+(-2)(-1)=5=5^{\frac{2}{2}}
\end{gathered}
$$

then follows

$$
G\left(\zeta_{3}\right) G\left(\zeta_{3}^{-1}\right)=5 \times 5=5^{2}
$$




\section{The quadratic Gauss sums}

The first Gauss sum ever computed is

$$
G_{2}=\sum_{r \bmod p}\left(\frac{r}{p}\right) \exp \left(\frac{2 \pi i r}{p}\right)
$$

where $p$ is an odd prime and $\left(\frac{r}{p}\right)$ is the unique quadratic character modulo $p$. We see at once that

$$
G_{2}^{2}=\sum_{r, s \bmod p}\left(\frac{r s}{p}\right) \exp \left(\frac{2 \pi i(r+s)}{p}\right)=\sum_{t \bmod p}\left(\frac{t}{p}\right) \sum_{s \bmod p} \exp \left(\frac{2 \pi i s(t+1)}{p}\right),
$$

under the substitution $r \equiv$ st $\bmod p$, and the inner sum is $p-1$ if $t \equiv-1 \bmod p$ and -1 otherwise. We therefore obtain

$$
G_{2}^{2}=(-1)^{\frac{p-1}{2}} p
$$

The problem here is to determine the right sign for $G_{2}$. Indeed

Theorem 6.1. For the quadratic Gauss sum, we have the following result:

$$
G_{2}=\left\{\begin{array}{l}
p^{1 / 2} \text { if } p \equiv 1 \bmod 4 \\
\text { ip } p^{1 / 2} \text { if } p \equiv-1 \bmod 4
\end{array},\right.
$$

where $p^{1 / 2}$ is the positive square root.

PROOF. There are many proofs of this theorem. We outline here that of Cauchy. Put $\zeta=\exp (2 \pi i / p)$.

If we consider the product

$$
\sigma_{2}=\prod_{r=1}^{(p-1) / 2}\left(\zeta^{2 r-1}-\zeta^{-2 r+1}\right)
$$

We then see the above product as follows. In the field $\mathbb{Q}$ the roots of unity we have are \pm 1 then $\sigma$ can be obtained just by selecting a half set modulo $p$ and build the product over this half set. If we choose as a half set to be the odd numbers modulo $p$ then a factor of this product $\zeta^{2 r-1}-\zeta^{-2 r+1}$ is seeing as $(1) \zeta^{2 r-1}+(-1) \zeta^{-2 r+1}$. Any other choice of another half set results in multiplying $\sigma_{2}$ by a suitable sign. Since the half set modulo $p,\{1,3, \cdots, p-2\}$ has as symmetric $\{-1,-3, \cdots,-p+2\}=$ $\{2,4, \cdots, p-1\}$ we therefore deduce that:

$$
\sigma_{2}^{2}=(-1)^{(p-1) / 2} \prod_{r=1}^{p-1}\left(\zeta^{r}-\zeta^{-r}\right)=(-1)^{p-1) / 2} p .
$$

this product is just the values of the cyclotomic polynomial of order $p$ at 1 , so that

$$
\sigma_{2}= \pm G_{2}
$$

In fact

$$
\sigma_{2}=G_{2}
$$


The sign of $\sigma_{2}^{2}$ is easily determined by writing it in the form

$$
\sigma_{2}=(2 i)^{(p-1) / 2} \prod_{r=1}^{\frac{p-1}{2}} \sin (2 \pi(2 r-1) / p)
$$

and then counting the number of negative factors in the product. Considering the congruence relations it could be enough to prove that $\sigma_{2}= \pm G_{2}$. The prime $p$ ramifies totally in $\mathbb{Q}(\zeta)$ and if $\lambda=1-\zeta$, we then have the prime ideal factorization $(p)=(\lambda)^{p-1}$. On the one hand we have $\left(\frac{r}{p}\right) \equiv r^{\frac{p-1}{2}} \bmod p$, so

$$
G_{2} \equiv \sum_{r \bmod p} r^{\frac{p-1}{2}}(1-\lambda)^{r}=\sum_{s=0}^{\frac{p-1}{2}}(-\lambda)^{s} \sum_{r \bmod p} r^{\frac{p-1}{2}}\left(\begin{array}{l}
r \\
s
\end{array}\right) \bmod \lambda^{\frac{p-1}{2}}
$$

However $\sum_{r \bmod p} r^{n}$ is either 0 or $-1 \bmod p$ according to whether $(p-1) \mid n$ or not, so that only the term $s=\frac{p-1}{2}$ contributes to the sum. By Wilson's Theorem, the result is

On the other hand, by similar arguments,

$$
G_{2} \equiv\left(\frac{p-1}{2}\right) ! \lambda^{\frac{p-1}{2}} \bmod \lambda^{\frac{p-1}{2}} .
$$

$$
\zeta^{2 r-1}-\zeta^{-2 r+1}=-\zeta^{-2 r+1}\left(1-\zeta^{2(2 r-1)}\right) \equiv-2(2 r-1) \lambda \bmod \lambda^{2},
$$

so that

$$
\sigma_{2} \equiv \prod_{r=1}^{\frac{p-1}{2}}(-2(2 r-1) \lambda) \equiv\left(\frac{p-1}{2}\right) ! \lambda^{\frac{p-1}{2}} \bmod \lambda^{\frac{p+1}{2}}
$$

Thus $G_{2} \equiv \sigma_{2} \bmod l a m b d a^{\frac{p-1}{2}}$. To finish the proof one has to determine the sign of $\sigma_{2}$.

$\sin \left(\frac{2(2 r-1) \pi}{p}\right)<0$ if $\frac{p+2}{2}<r \leq \frac{p-1}{2}$, therefore the product in $\sigma_{2}$ has $\frac{p-1}{2}-\left[\frac{p+2}{4}\right]$ negative terms and this is either $\frac{p-1}{4}$ or $\frac{p-3}{4}$ according to whether $p \equiv 1 \bmod 4$ or $p \equiv 3 \bmod 4$ respectively. We then deduce that the sign of $\sigma_{2}$ is $(i)^{\frac{p-1}{2}}(-1)^{\frac{p-1}{4}}=+1$ for $p \equiv 1 \bmod 4$ or $(i)^{\frac{p-1}{2}}(-1)^{\frac{p-3}{4}}=+i$ for $p \equiv i \bmod 4$

There are number of proofs of this theorem in the literature, $[\mathbf{J} . \mathbf{W} . \mathbf{S}]$.

Loxton attempted to generalize the relation between $G_{m}$ and $\sigma_{m}$, for higher order Gauss sums. He proved [Lox][Lox1] that there exits an algebraic integer $\alpha$ such that $\sigma_{m}=\alpha G_{m}$ The problem here to determine the magnitude of such $\alpha$. This question is still open. 


\section{Algorithm for the computation of the Gauss sums}

Let $K=\mathbb{Q}\left(\zeta_{m}\right)$ and $p$ prime integer such that $p^{f} \equiv 1 \bmod m$. Let $\pi \mid p$ be a prime such that $N_{K / \mathbb{Q}}(\pi)=p^{f} \equiv 1 \bmod (m)$

- 1. Find a primitive root modulo $\pi$

Let $g$ be a primitive root modulo $\pi$. That is , the cyclic group $\langle g\rangle$ generated by $g$ together with $\{0\}$ is a complete set of residues modulo $p$. Then if $x \in \mathbb{Z}\left[\zeta_{m}\right]$, there exists an integer $k>0$ so that $x=g^{k}$. In practice we just take a primitive root, whose absolute norm is the smallest, although this magnitude is related to the Riemann Hypothesis, our computation shows that they are relatively small. Th searching for a primitive root has a bad complexity and therefore the as $N(\pi) \rightarrow \infty$ the computation is very slow

- Computation of the Legendre symbol:

Put $N=\frac{p^{f}-1}{m}$ and $t=g^{N} \bmod \pi$ with $t^{m} \equiv 1(\bmod \pi)$.

Since $\pi$ is a polynom $f\left(\zeta_{m}\right)$ in $\zeta_{m}$ with integer coefficients, then test the power of $t$ such that $f\left(t^{\ell}\right) \equiv 0 \bmod \pi, 1 \leq \ell \leq m$, say $a$.

Evaluate $r=a^{-1} \bmod m$.

Define $\left(\frac{g}{\pi}\right)_{m}=\zeta_{m}^{r}$.

- Thus if $\chi=\chi_{\pi}=(\dot{\dot{\pi}})$ is the Dirichlet character associated to $\pi$, then its values at the non zero residue classes modulo $\pi$ are fully determined by its values at $g$.

We then notice that the determination of the values of the Gauss sums is related to the determination of the primitive roots $\bmod \pi$. At this stage the slower the algorithm of finding a primitive root, the slower the computation of the values of the gauss sums is.

From the Wilson's Theorem:

$$
g^{p^{f}-1} \equiv 1 \bmod \pi
$$

we have the following facts:

$$
\begin{aligned}
& -\quad g^{\frac{p^{f}-1}{2}} \equiv-1 \bmod \pi \\
& -\quad \chi\left(g^{a m}\right)=1 \\
& -\quad \chi\left(g^{a+m}\right)=\chi(a)
\end{aligned}
$$


Let $S^{*}=\left\{x_{1}, \cdots, x_{p^{f}-1}\right\}$ be any set of non zero residues modulo $\pi$, then

$$
\begin{aligned}
G(\chi, 1)=\sum_{k=1}^{p^{f}-1} \chi\left(g^{k}\right) e\left(g^{k}\right) & =\sum_{k=1}^{\frac{p^{f}-1}{2}} \chi\left(g^{k}\right)\left(e\left(g^{k}\right)+e\left(-g^{k}\right)\right) \\
& =2 \sum_{\ell=0}^{m-1} \sum_{k=1}^{\frac{p^{f}-1}{2 m}} \chi\left(g^{\ell}\right) \cos \left(2 \pi \operatorname{Tr}_{K / \mathbb{Q}}\left(\frac{g^{k m+\ell}}{\pi}\right)\right) \\
& =2 \sum_{\ell=0}^{m-1} \chi(g)^{\ell} \sum_{k=1}^{\frac{p^{f}-1}{2 m}} \cos \left(2 \pi \operatorname{Tr}_{K / \mathbb{Q}}\left(\frac{g^{k m+\ell}}{\pi}\right)\right) \\
& =\sum_{\ell=0}^{m-1} \chi(g)^{\ell} G_{\ell}(\pi)
\end{aligned}
$$

where

$G_{\ell}(\pi)=2 \sum_{k=1}^{\frac{p^{f}-1}{2 m}} \cos \left(2 \pi \operatorname{Tr}_{K / \mathbb{Q}}\left(\frac{g^{k m+\ell}}{\pi}\right)\right) ; \quad G_{\ell}(\pi)$ is called in appendix 1, a Gauss period .

$$
\text { If } \chi(g)=\zeta^{r}=\zeta_{m}^{r} \text { then }
$$

$$
G(\chi, 1)=\sum_{l=1}^{m-1} \zeta^{r l} G_{l}(\pi)
$$

Thus if $\varepsilon$ is any injection that maps $\mu_{m}(K)$ into $\mu_{m}(\mathbb{C})$ then

$$
\mathfrak{g}(1, \varepsilon, \pi)=G(\varepsilon \circ \chi, 1) \text {. }
$$

\section{Example}

$p=11 m=5 g=2$. Then $(11)=\mathfrak{p}_{1} \mathfrak{p}_{2} \mathfrak{p}_{3} \mathfrak{p}_{4}$ where $\mathfrak{p}_{i}=\left(11, \zeta_{5}-4^{i}\right)$.

One of these ideal factors has $\pi=-5 \zeta_{5}+16 \zeta_{5}^{2}-13 \zeta_{5}^{3}+13 \zeta_{5}^{4}$ as a uniformizer.

$(\pi)=\left(11, \zeta_{5}-5\right) . \mathbb{Z}\left[\zeta_{5}\right] /(\pi) \cong \mathbb{F}_{11}$, but $\mathbb{Z} /(11) \subset \mathbb{Z}\left[\zeta_{5}\right]$ implies that $\{0,1,2, \cdots, 10\}$ is a complete set of residues modulo $(\pi)$.

since

$$
e\left(\frac{x}{\pi}\right)=e\left(\frac{x \sigma(\pi) \sigma^{2}(\pi) \sigma^{3}(\pi)}{11}\right)
$$

and since $\operatorname{Tr}_{K / \mathbb{Q}}$ is $\mathbb{Q}$-linear we have

$$
\begin{aligned}
\operatorname{Tr}_{K / \mathbb{Q}}\left(\frac{x \sigma(\pi) \sigma^{2}(\pi) \sigma^{3}(\pi)}{11}\right) & =\frac{x}{11} \operatorname{Tr}_{K / \mathbb{Q}}\left(\sigma(\pi) \sigma^{2}(\pi) \sigma^{3}(\pi)\right) \\
& =\frac{x}{11} \operatorname{Tr}_{K / \mathbb{Q}}\left(23 \zeta_{5}-23 \zeta_{5}^{2}-74 \zeta_{5}^{3}-60 \zeta_{5}^{4}\right) \\
& =\frac{2 x}{11}
\end{aligned}
$$

therefore

$$
e\left(\frac{x}{\pi}\right)=\exp \left(\frac{4 x \pi i}{11}\right) \text { and }\left(\frac{2}{\pi}\right)_{5}=\zeta_{5}^{3}
$$




$$
\begin{aligned}
G_{l}(\pi) & =2 \sum_{k=1}^{\frac{11-1}{10}} \cos \left(2 \pi \operatorname{Tr}_{K / \mathbb{Q}}\left(\frac{2^{5 k+l}}{\pi}\right)\right) \\
& =2 \cos \left(2 \pi \operatorname{Tr}_{K / \mathbb{Q}}\left(\frac{2^{5 k+l}}{\pi}\right)\right) \\
& =2 \cos \left(2.2 \pi\left(\frac{2^{5 k+l}}{11}\right)\right)
\end{aligned}
$$

the Gauss sums

$$
\begin{aligned}
G\left(\left(\frac{\dot{\bar{x}}}{\pi}\right)_{5}, \pi\right) & =\sum_{k=1}^{10}\left(\frac{2^{i}}{\pi}\right)_{5} \exp \left(\frac{4.2^{k} \cdot i \pi}{11}\right) \\
& =\sum_{k=1}^{10} \zeta_{5}^{2 k} \exp \left(\frac{4.2^{k} i \pi}{11}\right) \\
& =\sum_{l=1}^{4} \zeta_{5}^{2 l} 2 \cos \left(\frac{2^{5 l+1} \cdot 2 \pi}{11}\right) \\
& =2\left(\zeta_{5}^{2} \cos \left(\frac{2 \pi}{11}\right)+\left(\zeta_{5}^{4} \cos \left(\frac{10 \pi}{11}\right)+\zeta_{5} \cos \left(\frac{6 \pi}{11}\right)\right)\right. \\
& +2\left(\zeta_{5}^{3} \cos \left(\frac{8 \pi}{11}\right)+\zeta_{5}^{0} \cos \left(\frac{4 \pi}{11}\right)\right)
\end{aligned}
$$

Let's take $\varepsilon\left(\zeta_{5}\right)=\exp (2 i \pi / 5)$ and we find that

$$
\mathfrak{g}(\pi, \varepsilon, 1)=-0.1517145346686306275249044042+3.313152984691512984920560792 * i
$$

$$
p=31, \quad m=5, \quad g=3
$$

Then $(31)=\mathfrak{p}_{1} \mathfrak{p}_{2} \mathfrak{p}_{3} \mathfrak{p}_{4}$ where $\mathfrak{p}_{i}=\left(31, \zeta_{5}-16^{i}\right), \quad 1<i<5$.

One of these ideal factors has $\pi=-63 \zeta_{5}+5 \zeta_{5}^{2}-42 \zeta_{5}^{3}-34 \zeta_{5}^{4}$ as a uniformizer.

$(\pi)=\left(31, \zeta_{5}-8\right) . \mathbb{Z}\left[\zeta_{5}\right] /(\pi) \cong \mathbb{F}_{31}$ but $\mathbb{Z} /(31) \subset \mathbb{Z}\left[\zeta_{5}\right]$ implies that $\{0,1,2, \cdots, 30\}$ is a complete set of residues modulo $(\pi)$.

$e\left(\frac{x}{\pi}\right)=e\left(\frac{x \sigma(\pi) \sigma^{2}(\pi) \sigma^{3}(\pi)}{31}\right)$ and since $\operatorname{Tr}_{K / \mathbb{Q}}$ is $\mathbb{Q}$-linear we have

$$
\begin{aligned}
\operatorname{Tr}_{K / \mathbb{Q}}\left(\frac{x \sigma(\pi) \sigma^{2}(\pi) \sigma^{3}(\pi)}{31}\right) & =\frac{x}{31} \operatorname{Tr}_{K / \mathbb{Q}}\left(\sigma(\pi) \sigma^{2}(\pi) \sigma^{3}(\pi)\right) \\
& =\frac{x}{31} \operatorname{Tr}_{K / \mathbb{Q}}\left(-49 \zeta_{5}+58 \zeta_{5}^{2}+335 \zeta_{5}^{3}+237 \zeta_{5}^{4}\right) \\
& =\frac{-681 x}{31}
\end{aligned}
$$


therefore $e\left(\frac{x}{\pi}\right)=\exp \left(\frac{-2 x \pi i}{31}\right)$ and $\left(\frac{3}{\pi}\right)_{5}=\zeta_{5}^{3}$

$(3.20)_{l}(\pi)=2 \sum_{k=1}^{\frac{31-1}{10}} \cos \left(2 \pi \operatorname{Tr}_{K / \mathbb{Q}}\left(\frac{2^{5 k+l}}{\pi}\right)\right)$

$$
\begin{aligned}
& =2 \sum_{k=1}^{3} \cos \left(2 \pi \operatorname{Tr}_{K / \mathbb{Q}}\left(\frac{3^{5 k+l}}{\pi}\right)\right) \\
& =2\left(\cos \left(-2 \pi \frac{3^{5.1+l}}{31}\right)+\cos \left(-2 \pi \frac{3^{5.2+l}}{31}\right)+\cos \left(-2 \pi \frac{3^{5.3+l}}{31}\right)\right) \\
& =2\left(\cos \left(2 \pi \frac{3^{5+l}}{31}\right)+\cos \left(2 \pi \frac{3^{10+l}}{31}\right)+\cos \left(2 \pi \frac{3^{15+l}}{31}\right)\right)
\end{aligned}
$$

The Gauss sums

(3.24) $G\left(\left(\frac{\dot{\pi}}{\pi}\right)_{5}, \pi\right)=\sum_{k=1}^{30}\left(\frac{3^{i}}{\pi}\right)_{5} \exp \left(\frac{-2.3^{k} . i \pi}{31}\right)=\sum_{k=1}^{30} \zeta_{5}^{3 k} \exp \left(\frac{2.3^{k} i \pi}{31}\right)$

$$
\begin{aligned}
& =\sum_{l=1}^{5} \zeta_{5}^{3 l} 2 \sum_{k=1}^{3} \cos \left(\frac{3^{5 k+l} \cdot 2 \pi}{31}\right) \\
& =2\left(\zeta_{5}^{3}\left(\cos \left(\frac{6 \pi}{31}\right)+\cos \left(\frac{32 \pi}{31}\right)+\cos \left(\frac{26 \pi}{31}\right)\right)\right) \\
& +2\left(\zeta_{5}\left(\cos \left(\frac{18 \pi}{31}\right)+\cos \left(\frac{34 \pi}{31}\right)+\cos \left(\frac{16 \pi}{31}\right)\right)\right) \\
& +2\left(\zeta_{5}^{4}\left(\cos \left(\frac{54 \pi}{31}\right)+\cos \left(\frac{40 \pi}{31}\right)+\cos \left(\frac{48 \pi}{31}\right)\right)\right) \\
& +2\left(\zeta_{5}^{2}\left(\cos \left(\frac{38 \pi}{31}\right)+\cos \left(\frac{58 \pi}{31}\right)+\cos \left(\frac{20 \pi}{31}\right)\right)\right) \\
& +2\left(\zeta_{5}^{0}\left(\cos \left(\frac{52 \pi}{31}\right)+\cos \left(\frac{50 \pi}{31}\right)+\cos \left(\frac{60 \pi}{31}\right)\right)\right)
\end{aligned}
$$

For distinct values of $\varepsilon\left(\zeta_{5}\right)$ we obtain the following results $\varepsilon\left(\zeta_{5}\right)=\exp (2 i \pi / 5)$ $G(\pi, \varepsilon, 1)=5.226579352648107065417247109-1.919080058380184951993603231 \times I$ $\varepsilon\left(\zeta_{5}\right)=\exp (4 i \pi / 5)$

$G(\pi, \varepsilon, 1)=4.552416669473279118809518850-3.205542460723585071329278448 \times I$ $\varepsilon\left(\zeta_{5}\right)=\exp (6 i \pi / 5)$ $G(\pi, \varepsilon, 1)=4.552416669473279118809518850+3.205542460723585071329278448 \times I$ $\varepsilon\left(\zeta_{5}\right)=\exp (8 i \pi / 5)$ $G(\pi, \varepsilon, 1)=5.226579352648107065417247109+1.919080058380184951993603231 \times I$ 


\section{4. data}

This is just a sample of the output file we have. The algebraic numbers are given in the basis $\zeta, \cdots, \zeta^{4}$. On the rows, $v$ stands for a prime factor of $P$. Under $v$ there are successively four lines, each corresponding to a conjugate of primary $v^{*}$ up to a fifth power of a unit, $v^{*}$, the $(1-\zeta)$-adic extension of $v^{*}$, the value of Gauss sums as a complex number, the angle corresponding to the Gauss sum, and the root of unity $\Omega\left(S_{0}, v^{*}\right)$ obtained from the fundamental domain with respect to $S_{0}$. 


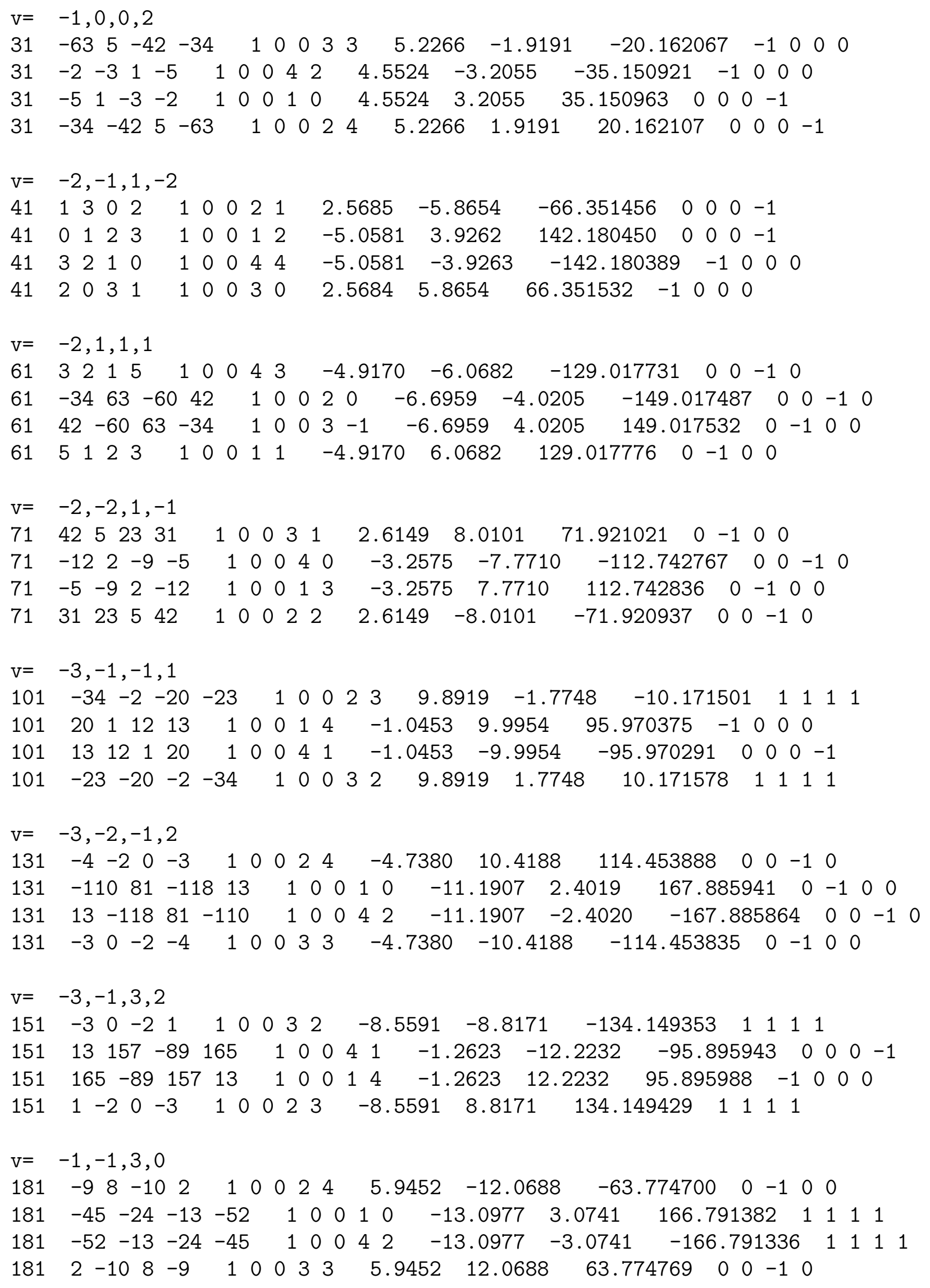




$$
\begin{aligned}
& \mathrm{v}=-3,2,-1,-2 \\
& \begin{array}{lllllllllllllllll}
191 & 71 & -2 & 45 & 42 & 1 & 0 & 0 & 2 & 1 & 5.1231 & 12.8356 & 68.241318 & -1 & 0 & 0 & 0
\end{array} \\
& \begin{array}{llllllllllllllllll}
191 & 10 & 6 & 2 & 13 & 1 & 0 & 0 & 1 & 2 & -6.3175 & 12.2918 & 117.201408 & 0 & 0 & -1 & 0
\end{array} \\
& \begin{array}{llllllllllllllllll}
191 & 13 & 2 & 6 & 10 & 1 & 0 & 0 & 4 & 4 & -6.3175 & -12.2918 & -117.201370 & 0 & -1 & 0 & 0
\end{array}
\end{aligned}
$$

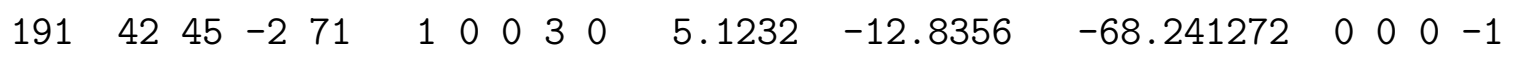

$$
\begin{aligned}
& \mathrm{v}=-2,0,0,3 \\
& \begin{array}{llllllllllllllllll}
211 & -1 & 9 & -6 & 9 & 1 & 0 & 0 & 0 & 2 & 5.7477 & -13.3403 & -66.690941 & 0 & 0 & -1 & 0
\end{array} \\
& \begin{array}{lllllllllllllllll}
211 & -16 & 34 & -31 & 24 & 1 & 0 & 0 & 0 & 2 & 12.8835 & 6.7093 & 27.509060 & -1 & 0 & 0 & 0
\end{array} \\
& \begin{array}{llllllllllllllllll}
211 & 24 & -31 & 34 & -16 & 1 & 0 & 0 & 0 & 2 & 12.8835 & -6.7093 & -27.508987 & 0 & 0 & 0 & -1
\end{array} \\
& \begin{array}{lllllllllllllllllll}
211 & 9 & -6 & 9 & -1 & 1 & 0 & 0 & 0 & 2 & 5.7477 & 13.3403 & 66.690987 & 0 & -1 & 0 & 0
\end{array} \\
& \mathrm{v}=-3,-3,-1,3 \\
& \begin{array}{lllllllllllllllll}
241 & -9 & -17 & 5 & -23 & 1 & 0 & 0 & 2 & 1 & -1.2352 & 15.4750 & 94.563652 & -1 & 0 & 0 & 0
\end{array} \\
& \begin{array}{llllllllllllllllll}
241 & -5 & -24 & 12 & -27 & 1 & 0 & 0 & 1 & 2 & 9.2728 & -12.4505 & -53.322437 & 0 & 0 & -1 & 0
\end{array} \\
& \begin{array}{lllllllllllllllll}
241 & -27 & 12 & -24 & -5 & 1 & 0 & 0 & 4 & 4 & 9.2728 & 12.4505 & 53.322475 & 0 & -1 & 0 & 0
\end{array} \\
& \begin{array}{llllllllllllllllll}
241 & -23 & 5 & -17 & -9 & 1 & 0 & 0 & 3 & 0 & -1.2352 & -15.4750 & -94.563622 & 0 & 0 & 0 & -1
\end{array} \\
& \mathrm{v}=-4,-3,0,-2 \\
& \begin{array}{lllllllllllllllll}
251 & -4 & 3 & -5 & 2 & 1 & 0 & 0 & 2 & 3 & -2.1786 & 15.6925 & 97.904053 & -1 & 0 & 0 & 0
\end{array} \\
& \begin{array}{llllllllllllllllllll}
251 & 60 & 81 & -13 & 118 & 1 & 0 & 0 & 1 & 4 & 14.4796 & -6.4298 & -23.944149 & 0 & 0 & -1 & 0
\end{array} \\
& \begin{array}{llllllllllllllllll}
251 & 118 & -13 & 81 & 60 & 1 & 0 & 0 & 4 & 1 & 14.4796 & 6.4298 & 23.944191 & 0 & -1 & 0 & 0
\end{array} \\
& \begin{array}{llllllllllllllllll}
251 & 2 & -5 & 3 & -4 & 1 & 0 & 0 & 3 & 2 & -2.1786 & -15.6925 & -97.904037 & 0 & 0 & 0 & -1
\end{array} \\
& \mathrm{v}=-4,-2,1,-4
\end{aligned}
$$

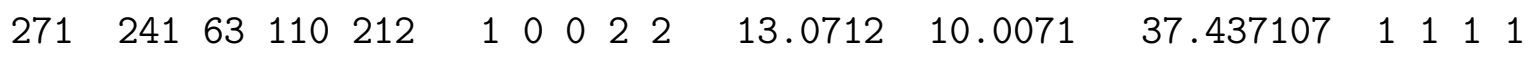

$$
\begin{aligned}
& \begin{array}{lllllllllllllllll}
271 & 0 & 1 & -3 & 3 & 1 & 0 & 0 & 1 & 3 & -9.4517 & 13.4783 & 125.040001 & -1 & 0 & 0 & 0
\end{array} \\
& \begin{array}{llllllllllllllllll}
271 & 3 & -3 & 1 & 0 & 1 & 0 & 0 & 4 & 0 & -9.4517 & -13.4784 & -125.039932 & 0 & 0 & 0 & -1
\end{array} \\
& \begin{array}{lllllllllllllllll}
271 & 212 & 110 & 63 & 241 & 1 & 0 & 0 & 3 & 1 & 13.0712 & -10.0071 & -37.437042 & 1 & 1 & 1 & 1
\end{array}
\end{aligned}
$$

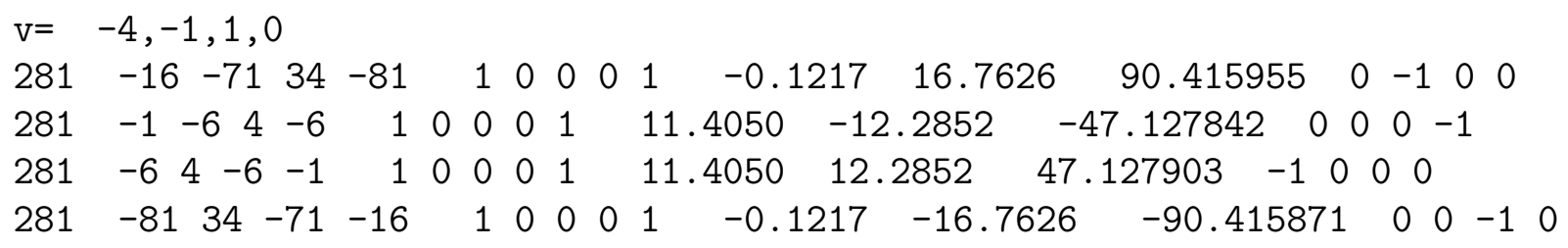

$$
\begin{aligned}
& \begin{array}{lllllllllllllllll}
\mathrm{v}= & -4, & -1,2,4 \\
311 & -4 & -2 & -5 & -3 & 1 & 0 & 0 & 2 & 0 & -0.3871 & -17.6309 & -91.257736 & 0 & -1 & 0 & 0
\end{array} \\
& \begin{array}{lllllllllllllllllll}
311 & 335 & 81 & 157 & 288 & 1 & 0 & 0 & 1 & 1 & 8.7925 & -15.2870 & -60.094284 & 1 & 1 & 1 & 1
\end{array} \\
& \begin{array}{lllllllllllllllll}
311 & 288 & 157 & 81 & 335 & 1 & 0 & 0 & 4 & 3 & 8.7924 & 15.2870 & 60.094360 & 1 & 1 & 1 & 1
\end{array} \\
& \begin{array}{llllllllllllllllll}
311 & -3 & -5 & -2 & -4 & 1 & 0 & 0 & 3 & 4 & -0.3871 & 17.6309 & 91.257812 & 0 & 0 & -1 & 0
\end{array} \\
& \mathrm{v}=-3,-3,3,4 \\
& \begin{array}{llllllllllllllllll}
331 & 0 & 6 & 7 & 3 & 1 & 0 & 0 & 1 & 0 & 0.2367 & 18.1919 & 89.254608 & 0 & -1 & 0 & 0
\end{array} \\
& \begin{array}{lllllllllllllllll}
331 & 7 & 0 & 3 & 6 & 1 & 0 & 0 & 3 & 3 & -12.1057 & 13.5813 & 131.712173 & 0 & -1 & 0 & 0
\end{array} \\
& \begin{array}{lllllllllllllllll}
331 & 6 & 3 & 0 & 7 & 1 & 0 & 0 & 2 & 4 & -12.1057 & -13.5813 & -131.712082 & 0 & 0 & -1 & 0
\end{array}
\end{aligned}
$$


118

$\begin{array}{llllllllllllllllll}331 & 3 & 7 & 6 & 0 & 1 & 0 & 0 & 4 & 2 & 0.2367 & -18.1919 & -89.254562 & 0 & 0 & -1 & 0\end{array}$

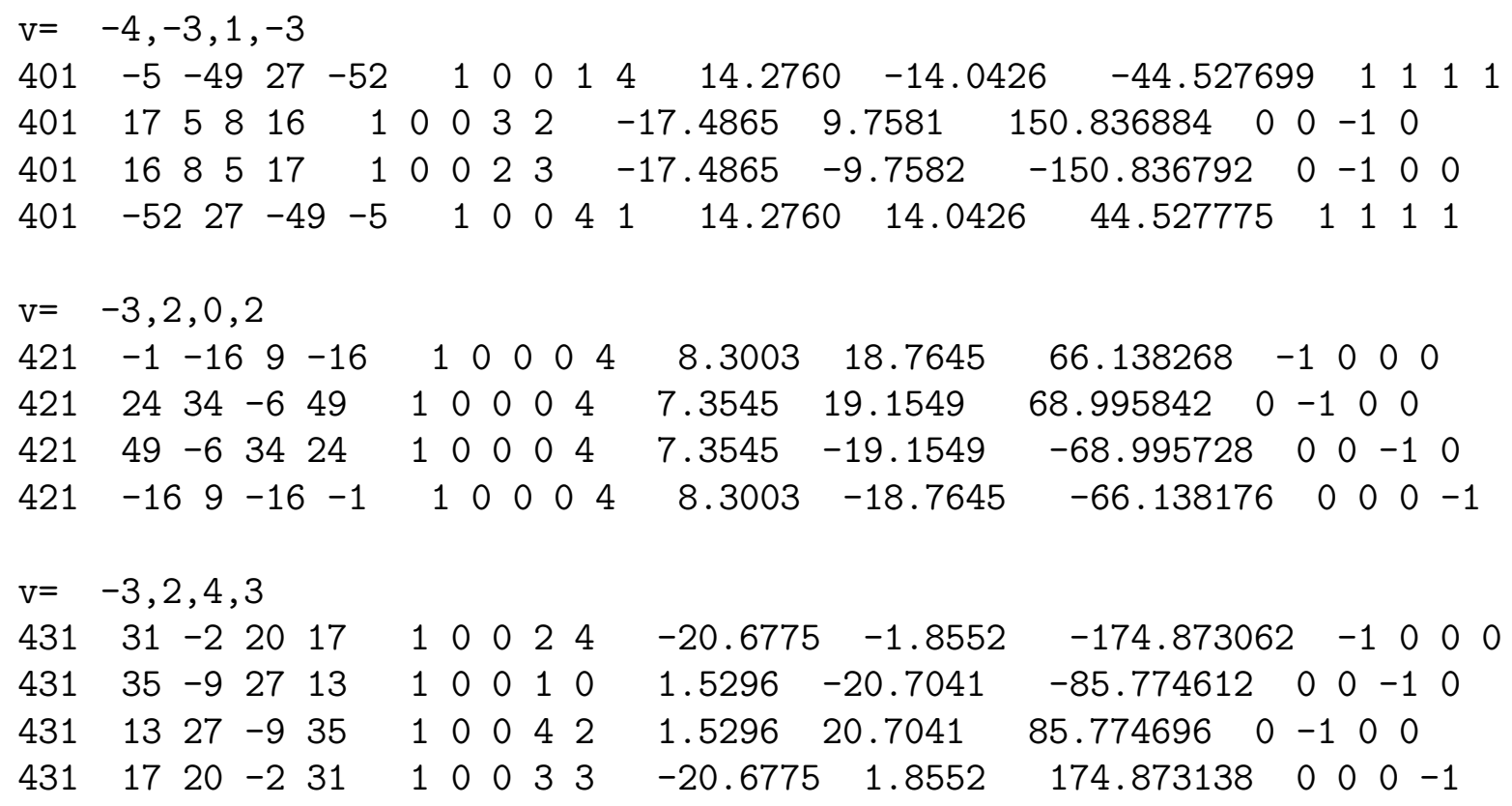




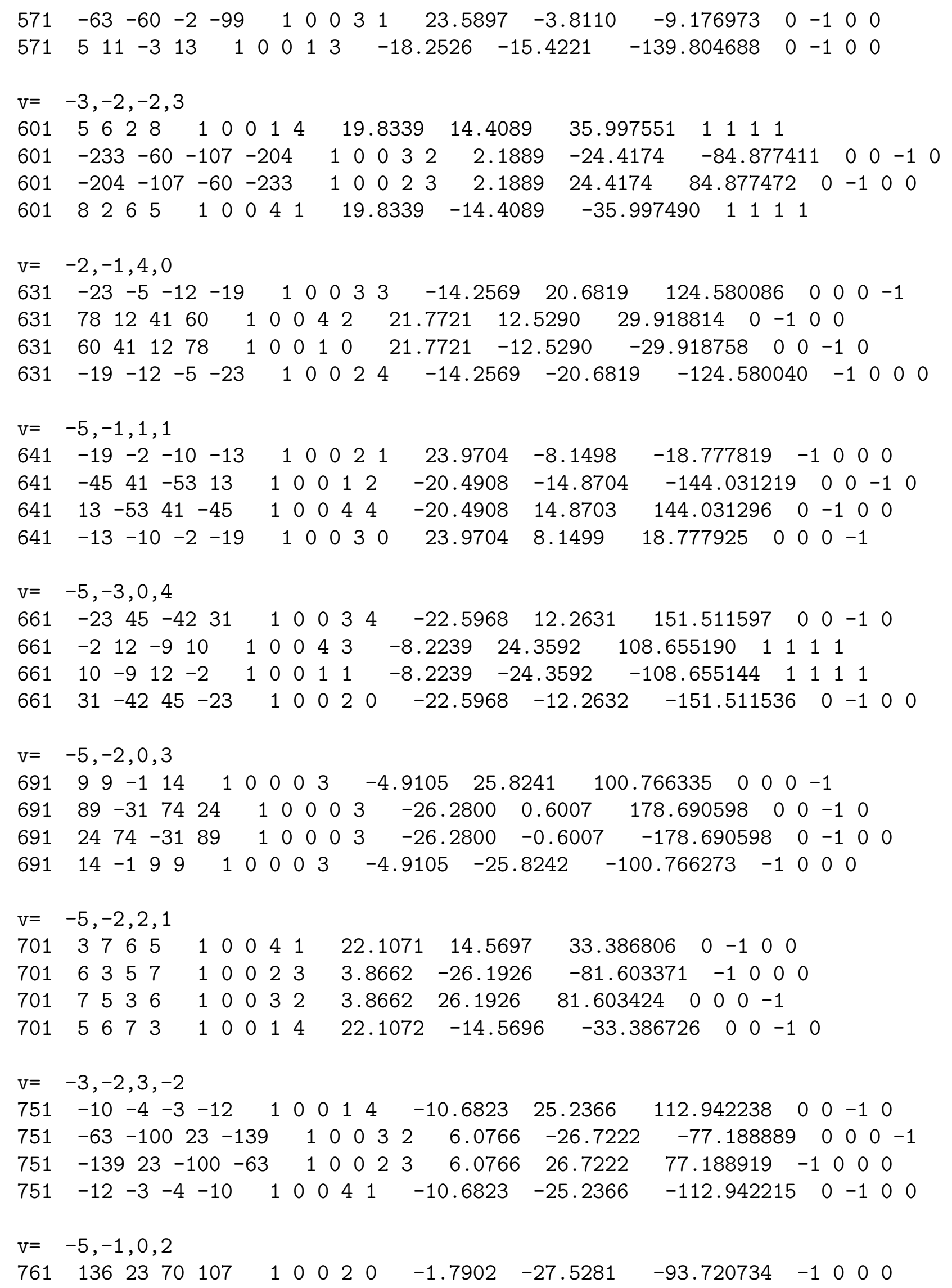


120

6. APPENDIX

$$
\begin{aligned}
& \begin{array}{llllllllllllllllll}
761 & -15 & -4 & -8 & -12 & 1 & 0 & 0 & 1 & 1 & 19.6068 & -19.4055 & -44.704449 & 0 & 0 & -1 & 0
\end{array} \\
& \begin{array}{llllllllllllllllll}
761 & -12 & -8 & -4 & -15 & 1 & 0 & 0 & 4 & 3 & 19.6068 & 19.4055 & 44.704472 & 0 & -1 & 0 & 0
\end{array} \\
& \begin{array}{lllllllllllllllll}
761 & 107 & 70 & 23 & 136 & 1 & 0 & 0 & 3 & 4 & -1.7902 & 27.5281 & 93.720779 & 0 & 0 & 0 & -1
\end{array}
\end{aligned}
$$

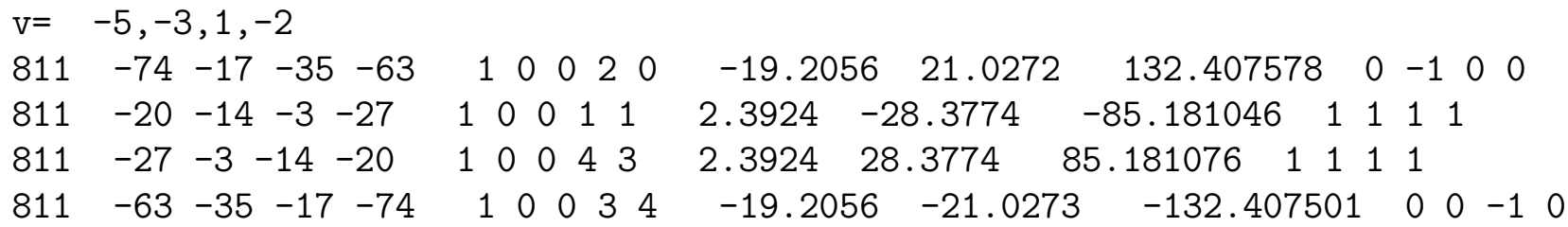

$$
\begin{aligned}
& \mathrm{v}=-5,2,-2,1 \\
& \begin{array}{llllllllllllllllll}
821 & -262 & -118 & -89 & -280 & 1 & 0 & 0 & 4 & 0 & -4.1373 & 28.3528 & 98.302040 & 0 & 0 & 0 & -1
\end{array} \\
& \begin{array}{llllllllllllllllll}
821 & 1 & -2 & 5 & -3 & 1 & 0 & 0 & 2 & 2 & 3.3161 & -28.4606 & -83.354042 & 1 & 1 & 1 & 1
\end{array} \\
& \begin{array}{llllllllllllllllll}
821 & -3 & 5 & -2 & 1 & 1 & 0 & 0 & 3 & 1 & 3.3161 & 28.4605 & 83.354073 & 1 & 1 & 1 & 1
\end{array} \\
& \begin{array}{lllllllllllllllll}
821 & -280 & -89 & -118 & -262 & 1 & 0 & 0 & 1 & 3 & -4.1372 & -28.3528 & -98.301994 & -1 & 0 & 0 & 0
\end{array} \\
& \mathrm{v}=-5,-4,0,5 \\
& \begin{array}{lllllllllllllllllll}
881 & 4 & 9 & 4 & -1 & 1 & 0 & 0 & 0 & 1 & 28.8634 & -6.9211 & -13.484297 & 0 & -1 & 0 & 0
\end{array} \\
& \begin{array}{lllllllllllllllllll}
881 & 4 & 4 & -1 & 9 & 1 & 0 & 0 & 0 & 1 & 23.6963 & -17.8741 & -37.027210 & 0 & 0 & 0 & -1
\end{array} \\
& \begin{array}{lllllllllllllllllll}
881 & 9 & -1 & 4 & 4 & 1 & 0 & 0 & 0 & 1 & 23.6963 & 17.8742 & 37.027328 & -1 & 0 & 0 & 0
\end{array} \\
& \begin{array}{lllllllllllllllllllll}
881 & -1 & 4 & 9 & 4 & 1 & 0 & 0 & 0 & 1 & 28.8634 & 6.9212 & 13.484399 & 0 & 0 & -1 & 0
\end{array}
\end{aligned}
$$




$\begin{array}{lllllllllllllllllll}1021 & -19 & 13 & -20 & 2 & 1 & 0 & 0 & 2 & 2 & -18.6410 & -25.9521 & -125.689102 & 0 & 0 & -1 & 0 \\ 1021 & 20 & 41 & -13 & 53 & 1 & 0 & 0 & 1 & 3 & -1.1150 & -31.9336 & -91.999771 & 0 & -1 & 0 & 0 \\ 1021 & 53 & -13 & 41 & 20 & 1 & 0 & 0 & 4 & 0 & -1.1151 & 31.9336 & 91.999832 & 0 & 0 & -1 & 0 & \\ 1021 & 2 & -20 & 13 & -19 & 1 & 0 & 0 & 3 & 1 & -18.6410 & 25.9521 & 125.689171 & 0 & -1 & 0 & 0\end{array}$

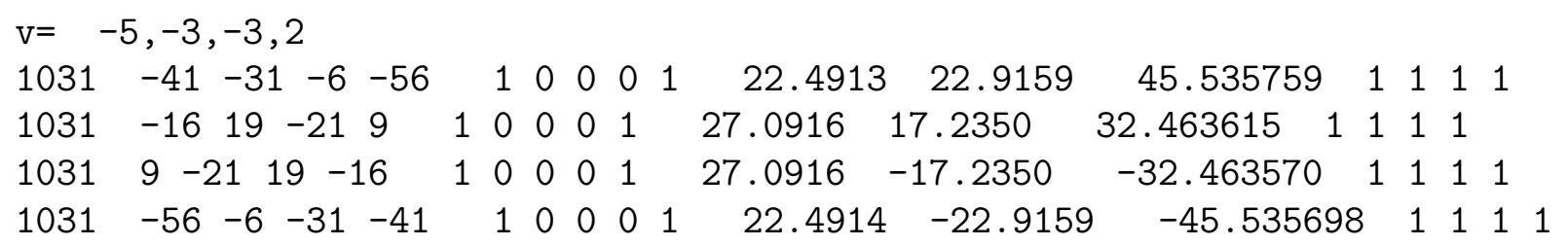


$\mathrm{v}=-4,-3,3,0$

$\begin{array}{lllllllllllllllll}1201 & 0 & -4 & -3 & 3 & 1 & 0 & 0 & 1 & 4 & -20.1002 & 28.2309 & 125.450554 & 0 & -1 & 0 & 0\end{array}$

$\begin{array}{llllllllllllllllll}1201 & -3 & 0 & 3 & -4 & 1 & 0 & 0 & 3 & 2 & 11.0004 & -32.8632 & -71.492851 & 0 & -1 & 0 & 0\end{array}$

$\begin{array}{llllllllllllllllll}1201 & -4 & 3 & 0 & -3 & 1 & 0 & 0 & 2 & 3 & 11.0004 & 32.8632 & 71.492897 & 0 & 0 & -1 & 0\end{array}$

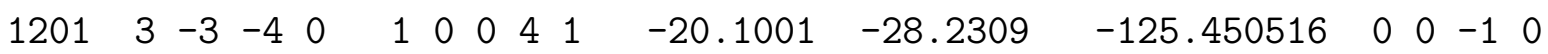

$\mathrm{v}=-3,-1,0,5$

$\begin{array}{lllllllllllllllll}1231 & 0 & -4 & 2 & -7 & 1 & 0 & 0 & 1 & 0 & 17.2045 & -30.5778 & -60.635899 & 0 & 0 & 0 & -1\end{array}$

$\begin{array}{lllllllllllllllll}1231 & 42 & -165 & 128 & -139 & 1 & 0 & 0 & 3 & 3 & 32.6080 & 12.9508 & 21.661312 & -1 & 0 & 0 & 0\end{array}$

$\begin{array}{lllllllllllllllll}1231 & -139 & 128 & -165 & 42 & 1 & 0 & 0 & 2 & 4 & 32.6080 & -12.9507 & -21.661264 & 0 & 0 & 0 & -1\end{array}$

$\begin{array}{lllllllllllllllllll}1231 & -7 & 2 & -4 & 0 & 1 & 0 & 0 & 4 & 2 & 17.2045 & 30.5779 & 60.635956 & -1 & 0 & 0 & 0\end{array}$

$\mathrm{v}=-6,-2,1,-2$

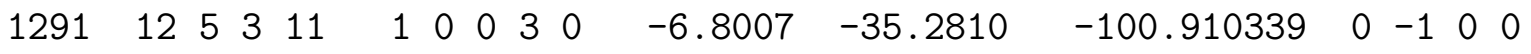

$\begin{array}{lllllllllllllllllll}1291 & 118 & -78 & 121 & -5 & 1 & 0 & 0 & 4 & 4 & 0.3337 & 35.9289 & 89.467789 & 0 & 0 & -1 & 0\end{array}$

$\begin{array}{lllllllllllllllll}1291 & -5 & 121 & -78 & 118 & 1 & 0 & 0 & 1 & 2 & 0.3338 & -35.9289 & -89.467743 & 0 & -1 & 0 & 0\end{array}$

$\begin{array}{llllllllllllllllll}1291 & 11 & 3 & 5 & 12 & 1 & 0 & 0 & 2 & 1 & -6.8007 & 35.2810 & 100.910423 & 0 & 0 & -1 & 0\end{array}$

$\mathrm{v}=-6,-4,-2,3$

$\begin{array}{lllllllllllllllll}1301 & 46 & 13 & 20 & 42 & 1 & 0 & 0 & 2 & 3 & 0.2905 & -36.0682 & -89.538460 & 1 & 1 & 1 & 1\end{array}$

$\begin{array}{lllllllllllllllll}1301 & 35 & 31 & 2 & 53 & 1 & 0 & 0 & 1 & 4 & -32.8079 & -14.9881 & -155.447037 & -1 & 0 & 0 & 0\end{array}$

$\begin{array}{lllllllllllllllll}1301 & 53 & 2 & 31 & 35 & 1 & 0 & 0 & 4 & 1 & -32.8079 & 14.9881 & 155.447037 & 0 & 0 & 0 & -1\end{array}$

$\begin{array}{llllllllllllllllll}1301 & 42 & 20 & 13 & 46 & 1 & 0 & 0 & 3 & 2 & 0.2905 & 36.0682 & 89.538521 & 1 & 1 & 1 & 1\end{array}$

$$
\begin{aligned}
& \mathrm{v}=-6,1,-5,1 \\
& \begin{array}{llllllllllllllllll}
1321 & 78 & -53 & 81 & -5 & 1 & 0 & 0 & 4 & 0 & -23.4438 & -27.7739 & -130.167496 & 0 & 0 & -1 & 0
\end{array} \\
& \begin{array}{lllllllllllllllll}
1321 & -4 & -12 & 5 & -13 & 1 & 0 & 0 & 2 & 2 & -1.7424 & -36.3038 & -92.747849 & 0 & 0 & -1 & 0
\end{array} \\
& \begin{array}{llllllllllllllllllll}
1321 & -13 & 5 & -12 & -4 & 1 & 0 & 0 & 3 & 1 & -1.7425 & 36.3038 & 92.747910 & 0 & -1 & 0 & 0
\end{array} \\
& \begin{array}{llllllllllllllllll}
1321 & -5 & 81 & -53 & 78 & 1 & 0 & 0 & 1 & 3 & -23.4438 & 27.7739 & 130.167480 & 0 & -1 & 0 & 0
\end{array} \\
& \mathrm{v}=-5,-1,-1,3 \\
& \begin{array}{lllllllllllllllll}
1361 & -24 & -7 & -10 & -23 & 1 & 0 & 0 & 2 & 0 & -16.9155 & 32.7852 & 117.291397 & 0 & 0 & 0 & -1
\end{array} \\
& \begin{array}{llllllllllllllllll}
1361 & -45 & -64 & 12 & -92 & 1 & 0 & 0 & 1 & 1 & 3.2801 & -36.7456 & -84.899002 & 0 & 0 & 0 & -1
\end{array} \\
& \begin{array}{lllllllllllllllll}
1361 & -92 & 12 & -64 & -45 & 1 & 0 & 0 & 4 & 3 & 3.2801 & 36.7456 & 84.898979 & -1 & 0 & 0 & 0
\end{array} \\
& \begin{array}{lllllllllllllllll}
1361 & -23 & -10 & -7 & -24 & 1 & 0 & 0 & 3 & 4 & -16.9154 & -32.7852 & -117.291367 & -1 & 0 & 0 & 0
\end{array}
\end{aligned}
$$

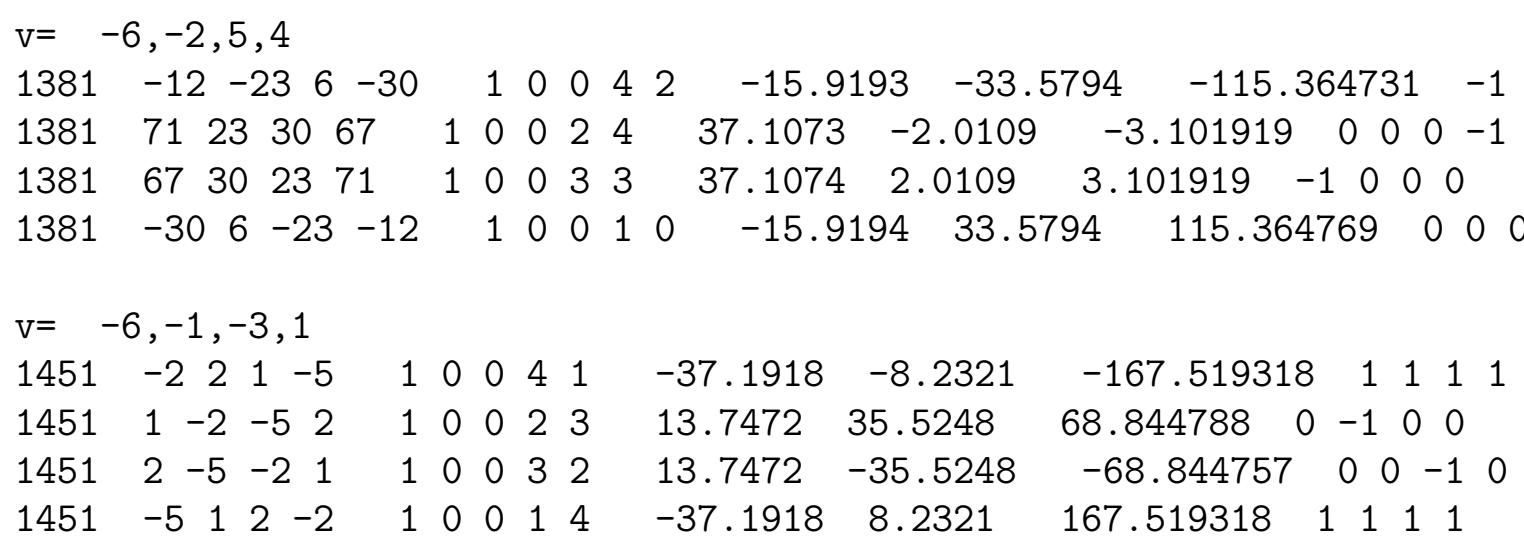




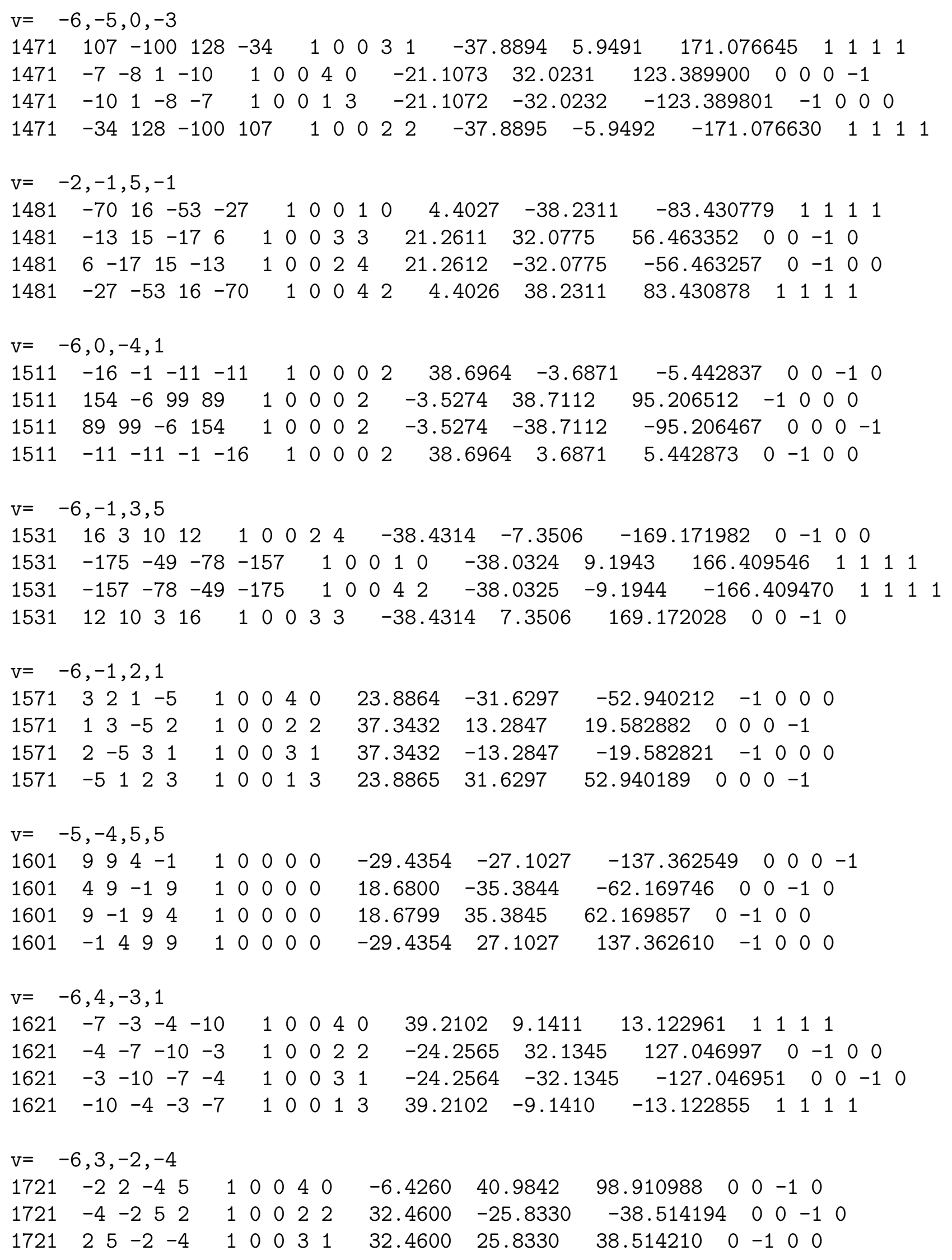




$$
\begin{aligned}
& \begin{array}{lllllllllllllllll}
1721 & 5 & -4 & 2 & -2 & 1 & 0 & 0 & 1 & 3 & -6.4260 & -40.9842 & -98.910904 & 0 & -1 & 0 & 0
\end{array} \\
& \mathrm{v}=-3,-3,4,-2 \\
& \begin{array}{lllllllllllllllll}
1741 & 31 & 63 & -20 & 82 & 1 & 0 & 0 & 2 & 1 & 36.7811 & 19.7015 & 28.175434 & 0 & -1 & 0 & 0
\end{array} \\
& \begin{array}{llllllllllllllllll}
1741 & 20 & -9 & 17 & 3 & 1 & 0 & 0 & 1 & 2 & 29.1820 & 29.8230 & 45.622395 & 1 & 1 & 1 & 1
\end{array} \\
& \begin{array}{lllllllllllllllll}
1741 & 3 & 17 & -9 & 20 & 1 & 0 & 0 & 4 & 4 & 29.1820 & -29.8230 & -45.622360 & 1 & 1 & 1 & 1
\end{array} \\
& \begin{array}{lllllllllllllllll}
1741 & 82 & -20 & 63 & 31 & 1 & 0 & 0 & 3 & 0 & 36.7811 & -19.7016 & -28.175470 & 0 & 0 & -1 & 0
\end{array} \\
& \mathrm{v}=-4,3,6,6 \\
& \begin{array}{lllllllllllllllll}
1801 & 6 & -67 & 45 & -63 & 1 & 0 & 0 & 2 & 3 & -30.4465 & 29.5637 & 135.842880 & 0 & -1 & 0 & 0
\end{array} \\
& \begin{array}{lllllllllllllllll}
1801 & 10 & 16 & -3 & 23 & 1 & 0 & 0 & 1 & 4 & -34.6339 & -24.5254 & -144.696411 & 1 & 1 & 1 & 1
\end{array} \\
& \begin{array}{lllllllllllllllll}
1801 & 23 & -3 & 16 & 10 & 1 & 0 & 0 & 4 & 1 & -34.6339 & 24.5253 & 144.696533 & 1 & 1 & 1 & 1
\end{array} \\
& \begin{array}{lllllllllllllllllll}
1801 & -63 & 45 & -67 & 6 & 1 & 0 & 0 & 3 & 2 & -30.4465 & -29.5637 & -135.842819 & 0 & 0 & -1 & 0
\end{array}
\end{aligned}
$$




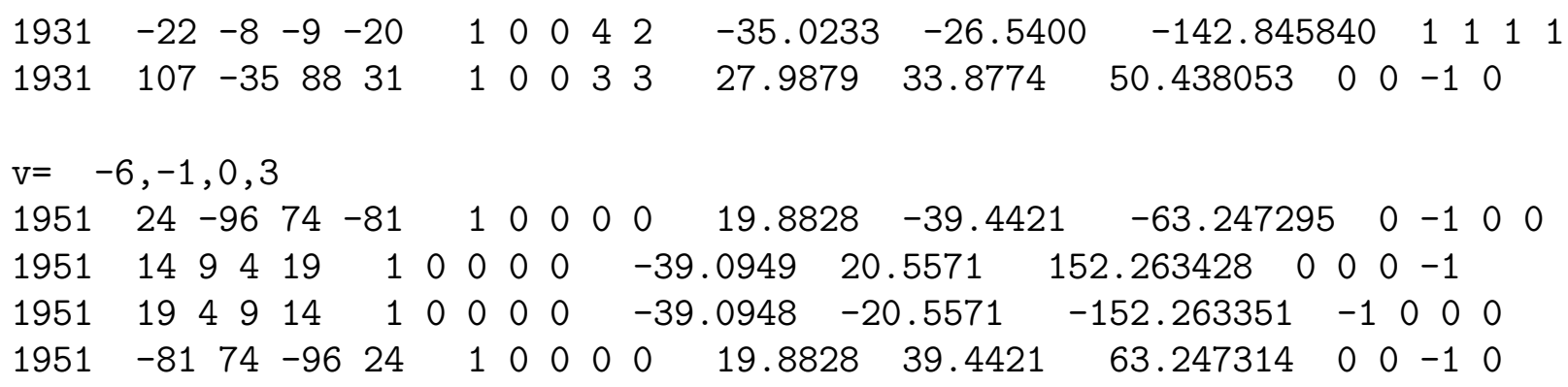


126

$\begin{array}{rrrrrrrrrr}2221 & 14 & 14 & -1 & 24 & 1 & 0 & 0 & 0 & 4 \\ 2221 & 24 & -1 & 14 & 14 & 1 & 0 & 0 & 0 & 4 \\ 2221 & 89 & 74 & 9 & 129 & 1 & 0 & 0 & 0 & 4\end{array}$

6. APPENDIX

$\begin{array}{llllllll}-22.7677 & 41.2629 & 118.888664 & 0 & 0 & 0 & -1 \\ -22.7677 & -41.2630 & -118.888596 & -1 & 0 & 0 & 0 \\ 26.5579 & -38.9317 & -55.699451 & 0 & 0 & -1 & 0\end{array}$

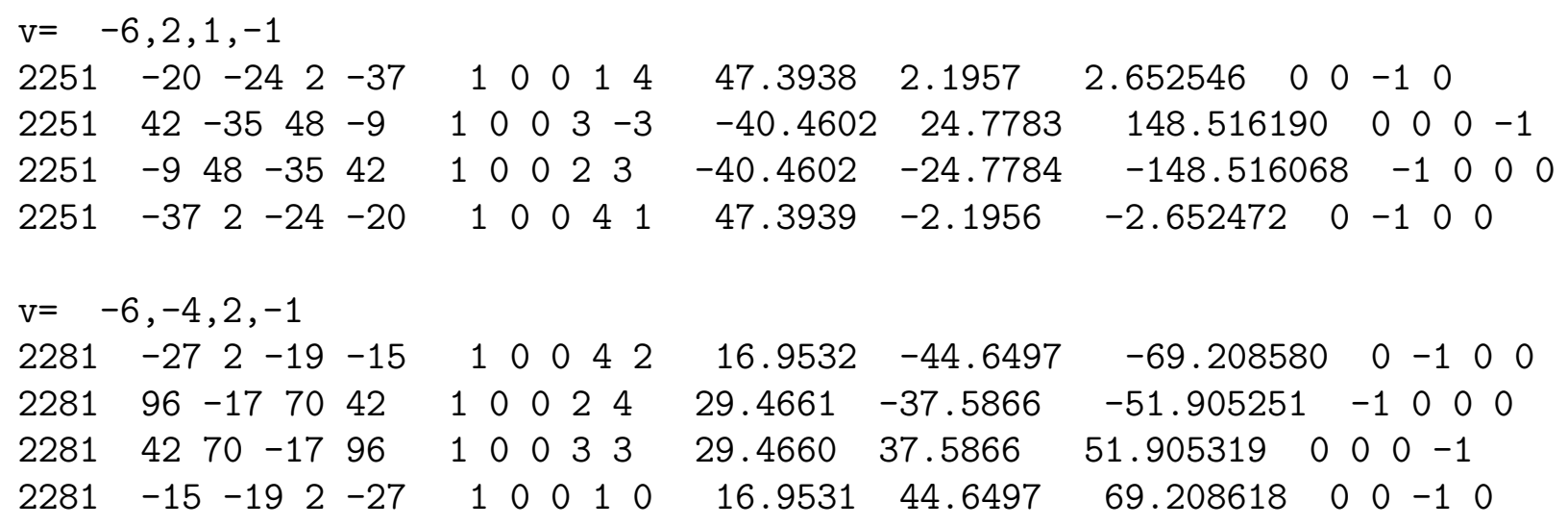




$\begin{array}{llllllllllllllllllllll}2411 & 31 & 48 & -10 & 67 & 1 & 0 & 0 & 2 & 0 & 39.2546 & 29.4970 & 36.922241 & 1 & 1 & 1 & 1 & & \\ 2411 & -45 & -9 & -23 & -37 & 1 & 0 & 0 & 1 & 1 & 12.4068 & -47.5086 & -75.364143 & -1 & 0 & 0 & 0 \\ 2411 & -37 & -23 & -9 & -45 & 1 & 0 & 0 & 4 & 3 & 12.4068 & 47.5086 & 75.364182 & 0 & 0 & 0 & -1 \\ 2411 & 67 & -10 & 48 & 31 & 1 & 0 & 0 & 3 & 4 & 39.2547 & -29.4970 & -36.922108 & 1 & 1 & 1 & 1\end{array}$

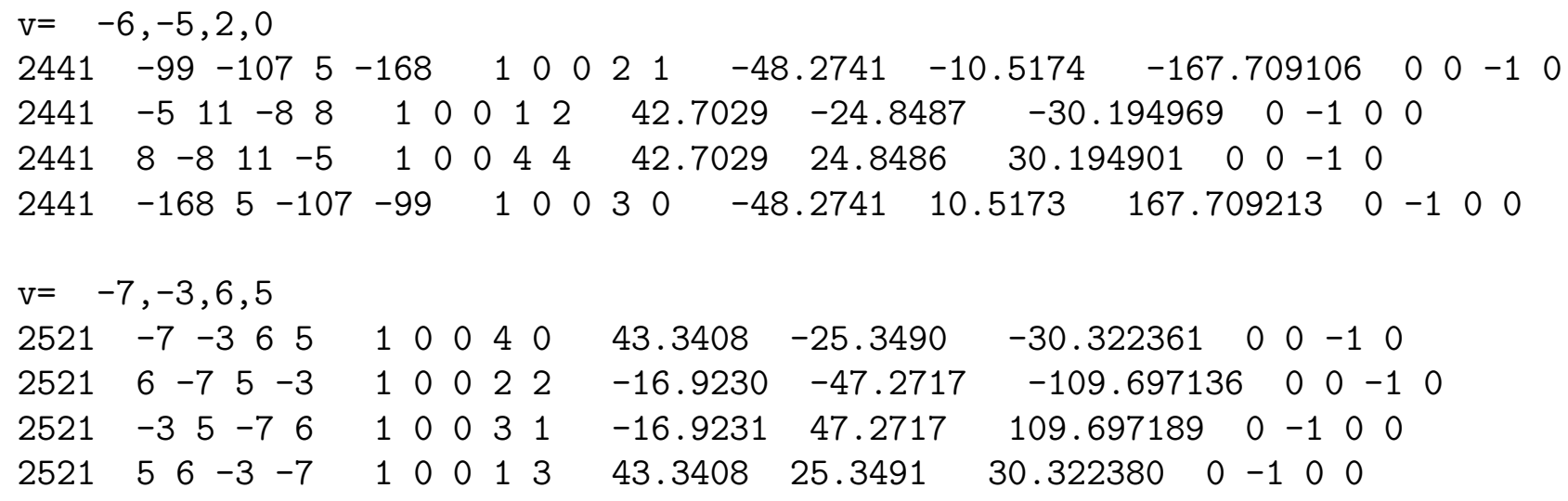


$\mathrm{v}=-5,-2,4,-1$

$\begin{array}{rllllllllllllllllll}2711 & 32 & 10 & 13 & 31 & 1 & 0 & 0 & 3 & 4 & 47.1471 & -22.0943 & -25.108921 & 0 & 0 & -1 & 0 \\ 2711 & 53 & 67 & -9 & 100 & 1 & 0 & 0 & 4 & 3 & 51.0471 & -10.2560 & -11.360162 & 1 & 1 & 1 & 1 \\ 2711 & 100 & -9 & 67 & 53 & 1 & 0 & 0 & 1 & 1 & 51.0471 & 10.2561 & 11.360266 & 1 & 1 & 1 & 1 & \\ 2711 & 31 & 13 & 10 & 32 & 1 & 0 & 0 & 2 & 0 & 47.1470 & 22.0943 & 25.108994 & 0 & -1 & 0 & 0\end{array}$

$\mathrm{v}=-7,-2,-2,2$

$\begin{array}{lllllllllllllllll}2731 & -12 & -3 & -9 & -10 & 1 & 0 & 0 & 4 & 2 & -34.0860 & 39.6125 & 130.711517 & 0 & 0 & 0 & -1\end{array}$

$\begin{array}{llllllllllllllllll}2731 & 306 & 23 & 175 & 212 & 1 & 0 & 0 & 2 & 4 & 46.8834 & 23.0857 & 26.215971 & 1 & 1 & 1 & 1\end{array}$

$\begin{array}{llllllllllllllllll}2731 & 212 & 175 & 23 & 306 & 1 & 0 & 0 & 3 & 3 & 46.8834 & -23.0857 & -26.215956 & 1 & 1 & 1 & 1\end{array}$

$\begin{array}{lllllllllllllllll}2731 & -10 & -9 & -3 & -12 & 1 & 0 & 0 & 1 & 0 & -34.0859 & -39.6125 & -130.711456 & -1 & 0 & 0 & 0\end{array}$

$\mathrm{v}=-7,-4,4,3$

$\begin{array}{lllllllllllllllll}2741 & -3 & -10 & -7 & 1 & 1 & 0 & 0 & 3 & 0 & 5.2820 & -52.0874 & -84.209641 & 0 & 0 & -1 & 0\end{array}$

$\begin{array}{lllllllllllllllll}2741 & -7 & -3 & 1 & -10 & 1 & 0 & 0 & 4 & 4 & -46.7768 & -23.5143 & -153.311707 & 1 & 1 & 1 & 1\end{array}$

$\begin{array}{rrrrrrrrrrrrrrrrr}2741 & -10 & 1 & -3 & -7 & 1 & 0 & 0 & 1 & 2 & -46.7769 & 23.5143 & 153.311798 & 1 & 1 & 1 & 1\end{array}$

$\begin{array}{llllllllllllllllll}2741 & 1 & -7 & -10 & -3 & 1 & 0 & 0 & 2 & 1 & 5.2819 & 52.0874 & 84.209724 & 0 & -1 & 0 & 0\end{array}$

$\mathrm{v}=-7,-1,2,2$

$\begin{array}{lllllllllllllllll}2791 & 67 & 5 & 38 & 46 & 1 & 0 & 0 & 3 & 0 & -29.6484 & 43.7262 & 124.139084 & 0 & 0 & -1 & 0\end{array}$

$\begin{array}{lllllllllllllllll}2791 & 28 & -23 & 31 & -5 & 1 & 0 & 0 & 4 & 4 & -41.5679 & 32.6053 & 141.889832 & 1 & 1 & 1 & 1\end{array}$

$\begin{array}{lllllllllllllllllllllll}2791 & -5 & 31 & -23 & 28 & 1 & 0 & 0 & 1 & 2 & -41.5679 & -32.6054 & -141.889801 & 1 & 1 & 1 & 1\end{array}$

$2791 \quad 46 \begin{array}{lllllllllllllllll}38 & 5 & 67 & 1 & 0 & 0 & 2 & 1 & -29.6483 & -43.7262 & -124.139030 & 0 & -1 & 0 & 0\end{array}$

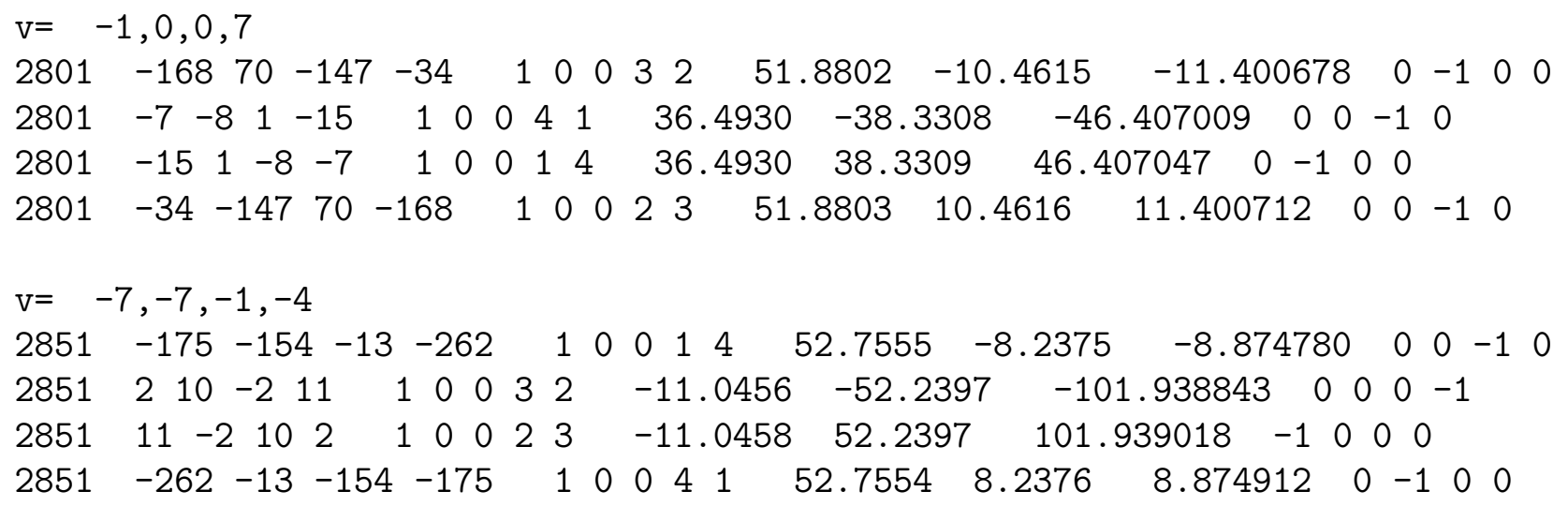




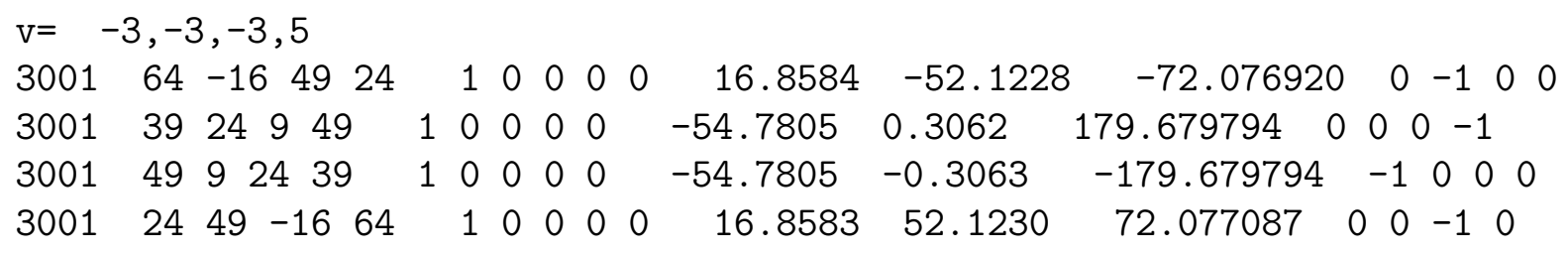


130

322

\section{APPENDIX}

$\begin{array}{llllllll}-53.7025 & 18.3588 & 161.126434 & 0 & -1 & 0 & 0\end{array}$

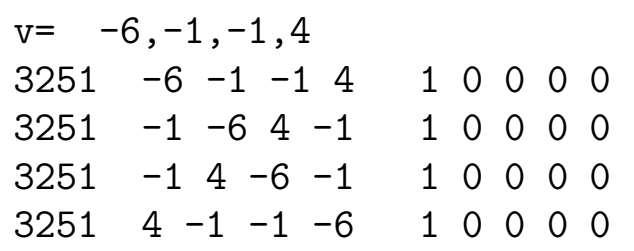

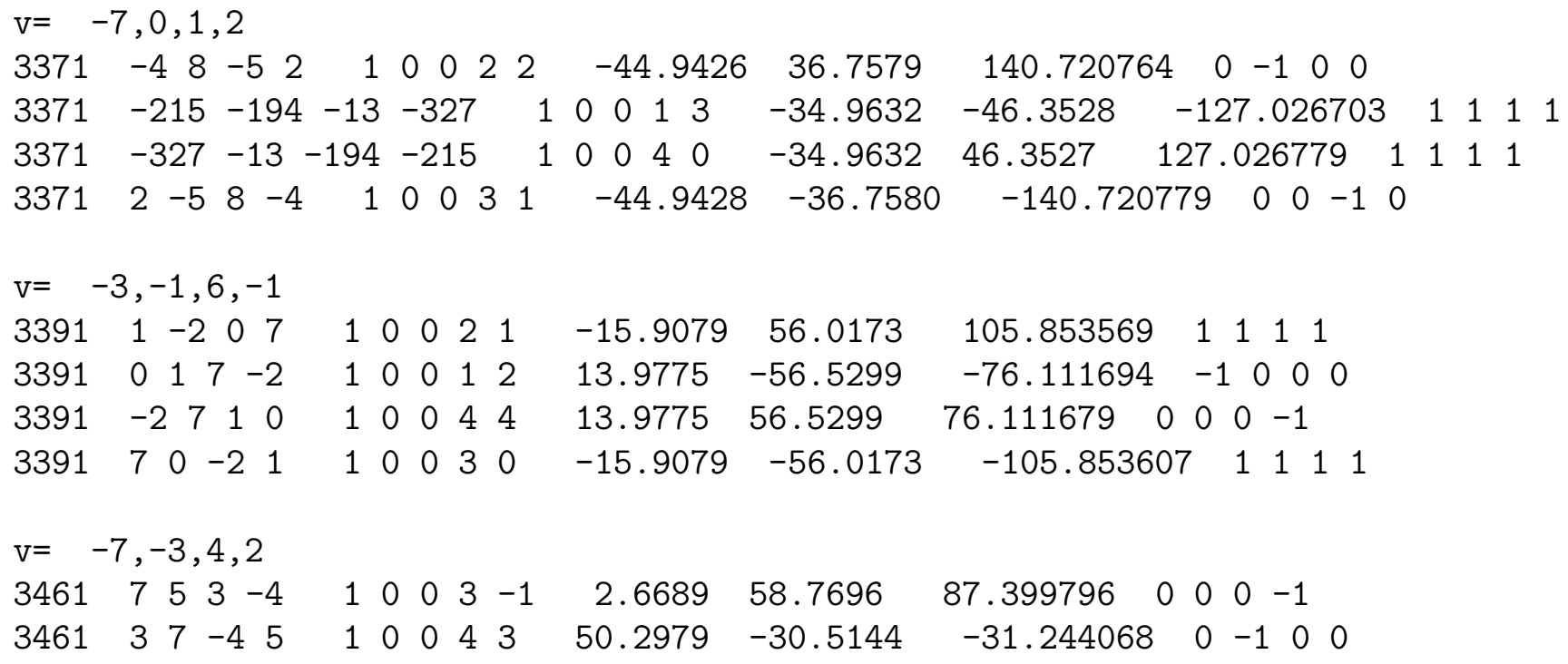




$\begin{array}{rrrrrrrrrrrrrrrrrrr}3461 & 5 & -4 & 7 & 3 & 1 & 0 & 0 & 1 & 1 & 50.2978 & 30.5144 & 31.244122 & 0 & 0 & -1 & 0 \\ 3461 & -4 & 3 & 5 & 7 & 1 & 0 & 0 & 2 & 0 & 2.6690 & -58.7696 & -87.399727 & -1 & 0 & 0 & 0\end{array}$

$\mathrm{v}=-7,-4,2,-5$

$\begin{array}{lllllllllllllllll}3491 & -23 & -85 & 38 & -99 & 1 & 0 & 0 & 3 & 0 & 54.9101 & 21.8148 & 21.667038 & 0 & 0 & -1 & 0\end{array}$ $\begin{array}{lllllllllllllllll}3491 & 28 & 12 & 11 & 30 & 1 & 0 & 0 & 4 & 4 & 19.1232 & -55.9044 & -71.115707 & 1 & 1 & 1 & 1\end{array}$ $\begin{array}{llllllllllllllllll}3491 & 30 & 11 & 12 & 28 & 1 & 0 & 0 & 1 & 2 & 19.1231 & 55.9045 & 71.115822 & 1 & 1 & 1 & 1\end{array}$ $\begin{array}{lllllllllllllllllll}3491 & -99 & 38 & -85 & -23 & 1 & 0 & 0 & 2 & 1 & 54.9101 & -21.8147 & -21.666927 & 0 & -1 & 0 & 0\end{array}$

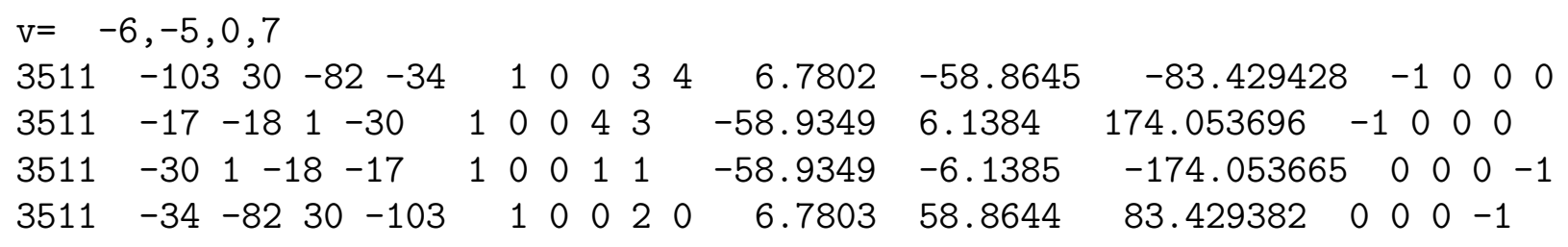




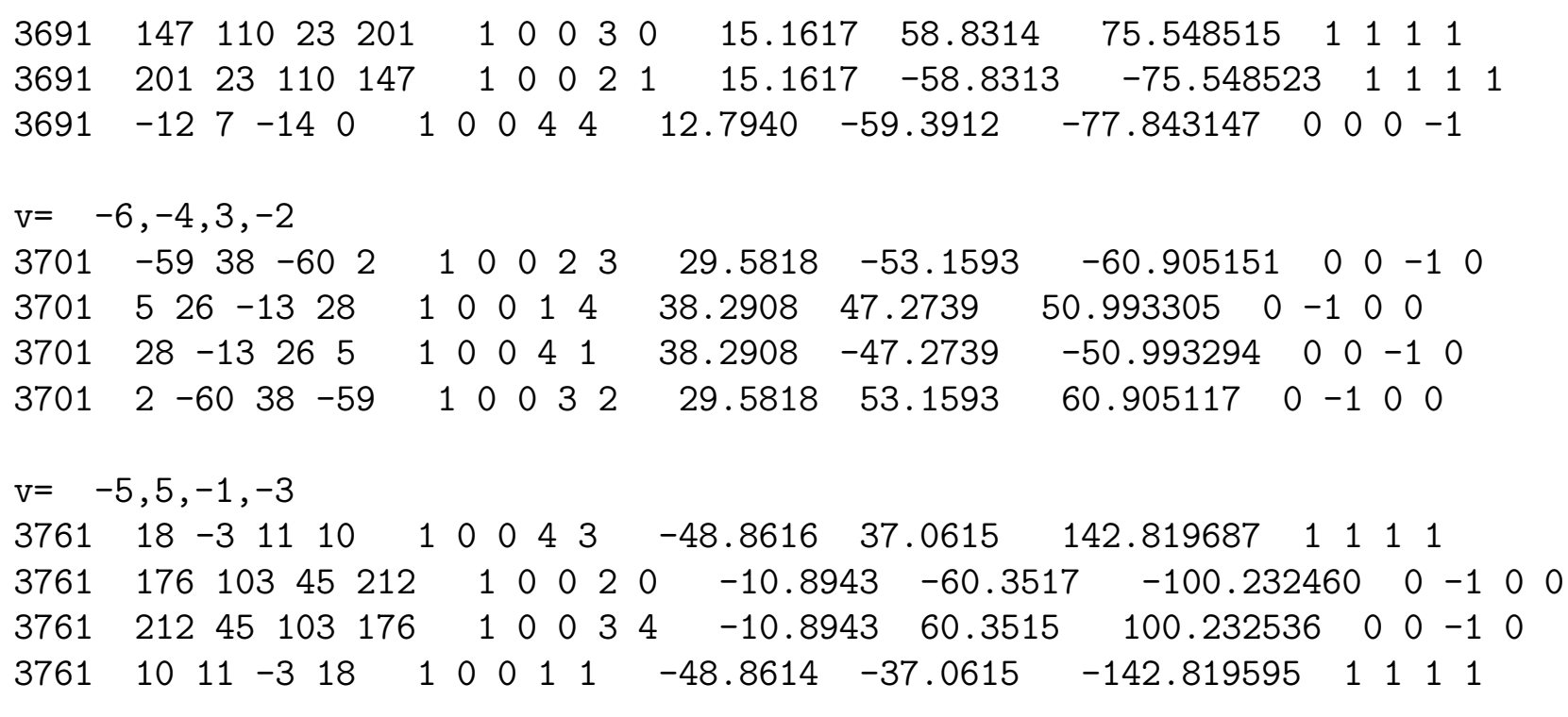




$\begin{array}{rllllllllllllllll}4001 & 2 & 0 & -7 & 1 & 1 & 0 & 0 & 3 & 2 & 61.8830 & 13.0954 & 11.948439 & 0 & -1 & 0 & 0 \\ 4001 & -7 & 2 & 1 & 0 & 1 & 0 & 0 & 4 & 1 & 48.9827 & 40.0212 & 39.250446 & 0 & 0 & -1 & 0 \\ 4001 & 0 & 1 & 2 & -7 & 1 & 0 & 0 & 1 & 4 & 48.9827 & -40.0212 & -39.250420 & 0 & -1 & 0 & 0 \\ 4001 & 1 & -7 & 0 & 2 & 1 & 0 & 0 & 2 & 3 & 61.8832 & -13.0954 & -11.948323 & 0 & 0 & -1 & 0\end{array}$

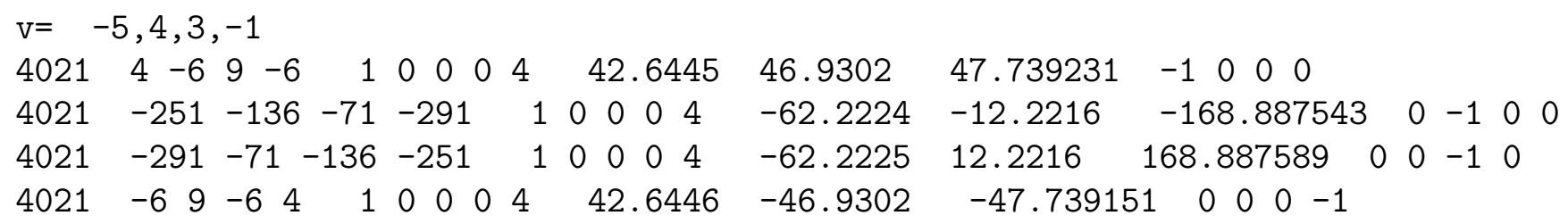




$$
\begin{aligned}
& \mathrm{v}=-6,-5,3,-1 \\
& \begin{array}{lllllllllllllllll}
4241 & -85 & -39 & -28 & -92 & 1 & 0 & 0 & 1 & 2 & 63.1509 & -15.9050 & -14.136335 & 1 & 1 & 1 & 1
\end{array} \\
& \begin{array}{lllllllllllllllll}
4241 & -38 & -25 & -7 & -49 & 1 & 0 & 0 & 3 & 0 & 65.1225 & -0.2137 & -0.187672 & 0 & 0 & -1 & 0
\end{array} \\
& \begin{array}{lllllllllllllllll}
4241 & -49 & -7 & -25 & -38 & 1 & 0 & 0 & 2 & 1 & 65.1227 & 0.2138 & 0.187672 & 0 & -1 & 0 & 0
\end{array} \\
& \begin{array}{lllllllllllllllll}
4241 & -92 & -28 & -39 & -85 & 1 & 0 & 0 & 4 & 4 & 63.1509 & 15.9051 & 14.136448 & 1 & 1 & 1 & 1
\end{array}
\end{aligned}
$$

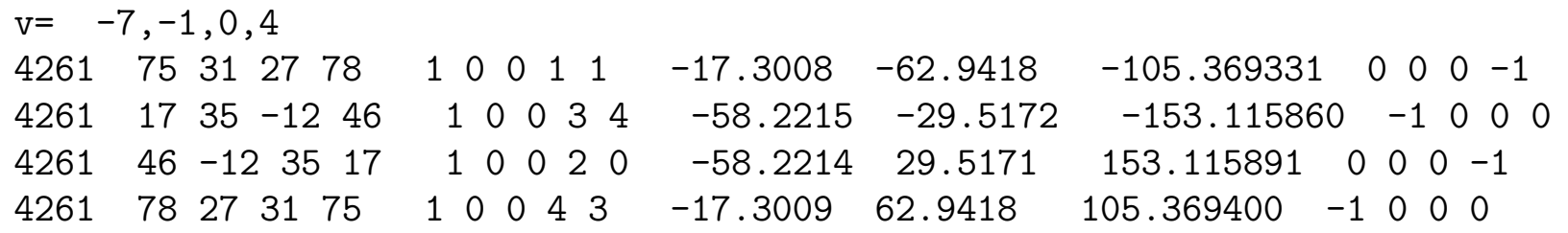




$$
\begin{aligned}
& \mathrm{v}=-8,-6,1,-6 \\
& \begin{array}{lllllllllllllllll}
4561 & 6 & -2 & 0 & 7 & 1 & 0 & 0 & 2 & 0 & 8.1615 & -67.0401 & -83.058891 & -1 & 0 & 0 & 0
\end{array} \\
& \begin{array}{llllllllllllllllll}
4561 & 0 & 6 & 7 & -2 & 1 & 0 & 0 & 1 & 1 & -59.7260 & -31.5245 & -152.174118 & 0 & 0 & -1 & 0
\end{array} \\
& \begin{array}{llllllllllllllllll}
4561 & -2 & 7 & 6 & 0 & 1 & 0 & 0 & 4 & 3 & -59.7262 & 31.5245 & 152.174149 & 0 & -1 & 0 & 0
\end{array} \\
& \begin{array}{llllllllllllllllll}
4561 & 7 & 0 & -2 & 6 & 1 & 0 & 0 & 3 & 4 & 8.1614 & 67.0403 & 83.059021 & 0 & 0 & 0 & -1
\end{array}
\end{aligned}
$$

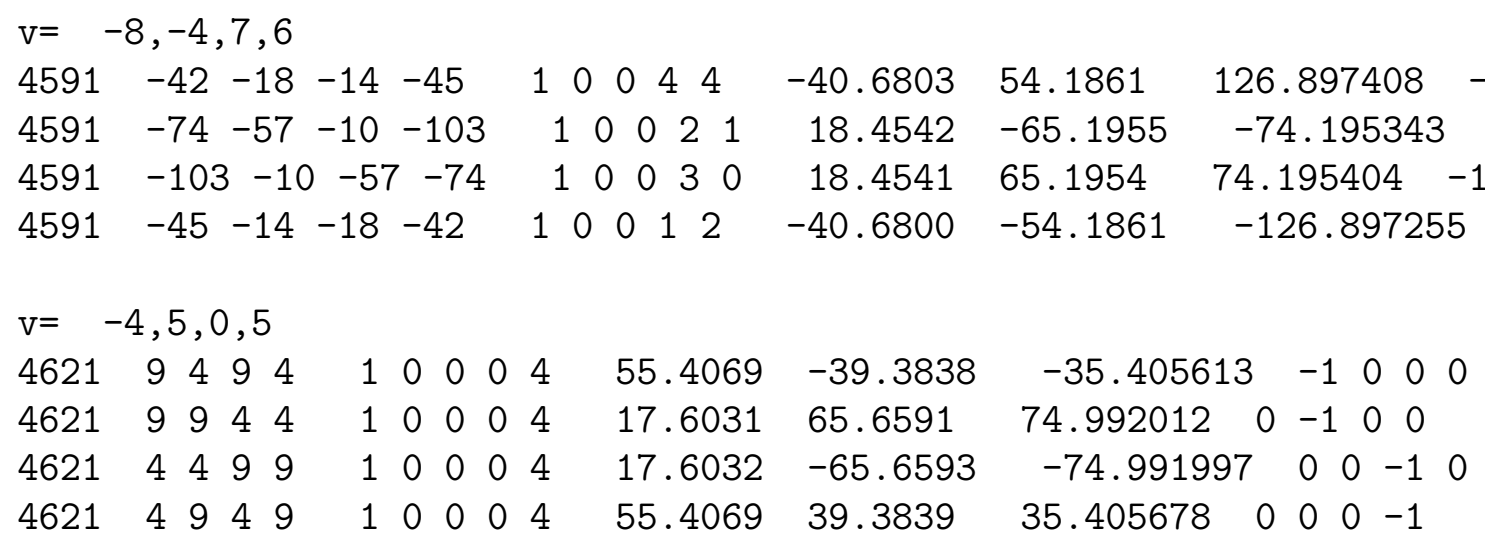




$$
\begin{aligned}
& \begin{array}{llllllllllllllllll}
4801 & -5 & -14 & -8 & -2 & 1 & 0 & 0 & 1 & 4 & 19.7151 & 66.4253 & 73.469086 & 0 & 0 & -1 & 0
\end{array} \\
& \mathrm{v}=-8,-8,-6,3 \\
& \begin{array}{lllllllllllllllll}
4831 & 16 & -32 & 30 & -23 & 1 & 0 & 0 & 2 & 4 & -8.1497 & 69.0260 & 96.733559 & -1 & 0 & 0 & 0
\end{array} \\
& \begin{array}{lllllllllllllllll}
4831 & -30 & -49 & 12 & -67 & 1 & 0 & 0 & 1 & 0 & 60.1292 & -34.8640 & -30.105925 & 0 & 0 & -1 & 0
\end{array} \\
& \begin{array}{llllllllllllllllll}
4831 & -67 & 12 & -49 & -30 & 1 & 0 & 0 & 4 & 2 & 60.1291 & 34.8639 & 30.105871 & 0 & -1 & 0 & 0
\end{array} \\
& \begin{array}{lllllllllllllllll}
4831 & -23 & 30 & -32 & 16 & 1 & 0 & 0 & 3 & 3 & -8.1496 & -69.0260 & -96.733490 & 0 & 0 & 0 & -1
\end{array}
\end{aligned}
$$

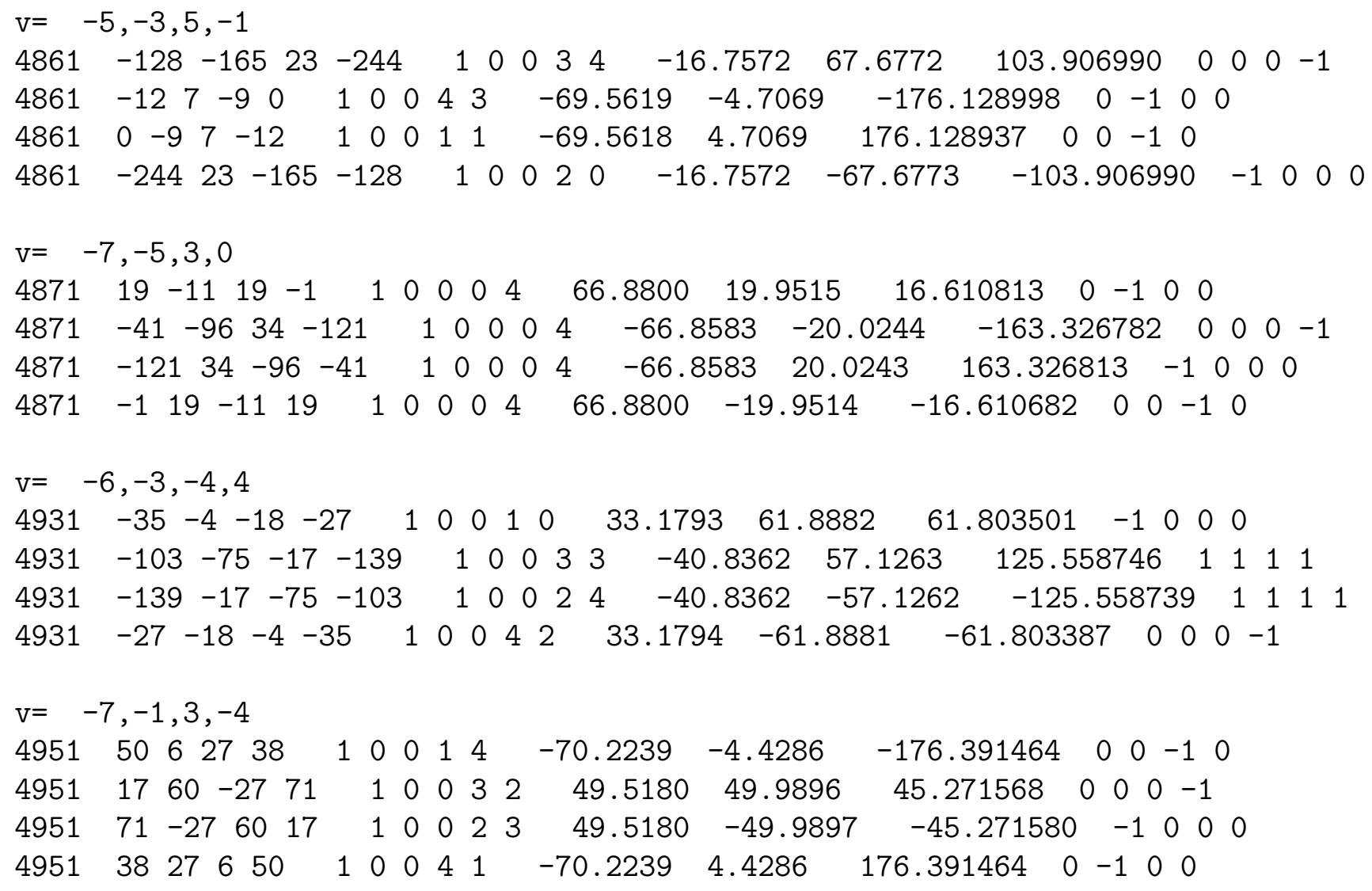


$\begin{array}{llllllllllllllllll}5051 & 28 & 7 & 11 & 25 & 1 & 0 & 0 & 4 & 1 & 34.4243 & -62.1769 & -61.028820 & 1 & 1 & 1 & 1\end{array}$ $\begin{array}{llllllllllllllllll}5051 & 147 & 85 & 38 & 176 & 1 & 0 & 0 & 3 & 2 & -58.9203 & 39.7418 & 146.000320 & 0 & 0 & -1 & 0\end{array}$

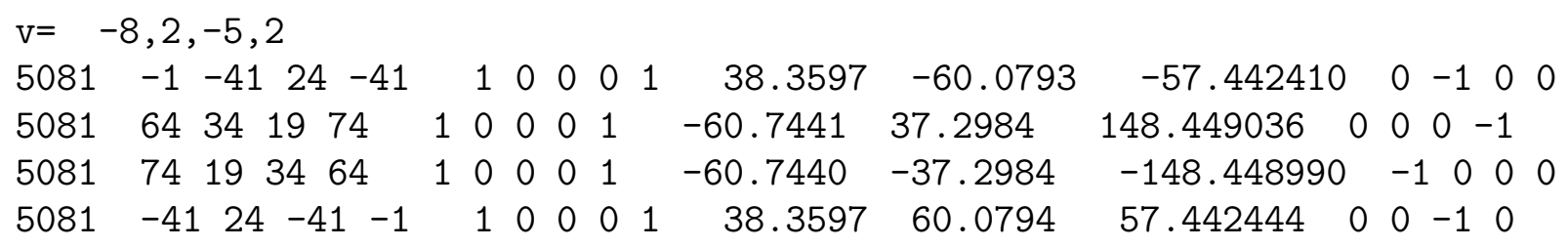


138

$$
\begin{aligned}
& \begin{array}{lllllllllllllllll}
5381 & 4 & 9 & -1 & 4 & 1 & 0 & 0 & 0 & 1 & 35.4470 & 64.2223 & 61.103809 & 0 & 0 & 0 & -1
\end{array} \\
& \begin{array}{llllllllllllllllll}
5381 & 4 & -1 & 9 & 4 & 1 & 0 & 0 & 0 & 1 & 35.4470 & -64.2224 & -61.103840 & -1 & 0 & 0 & 0
\end{array} \\
& \begin{array}{lllllllllllllllll}
5381 & -1 & 4 & 4 & 9 & 1 & 0 & 0 & 0 & 1 & 30.8147 & 66.5692 & 65.160751 & 0 & 0 & -1 & 0
\end{array}
\end{aligned}
$$

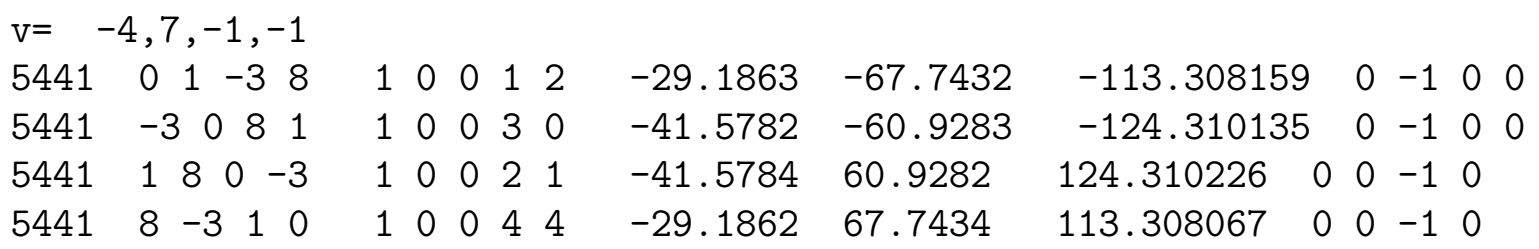

$\mathrm{v}=-8,-2,1,5$

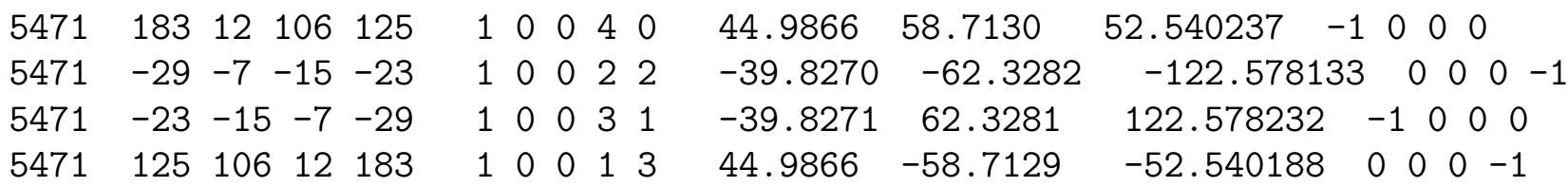

$\mathrm{v}=-8,-5,1,8$

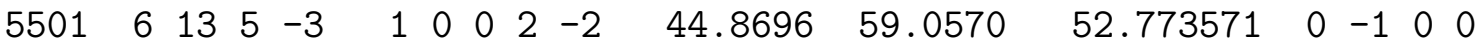

$\begin{array}{lllllllllllllllll}5501 & 5 & 6 & -3 & 13 & 1 & 0 & 0 & 1 & 4 & -29.3018 & 68.1352 & 113.270340 & 1 & 1 & 1 & 1\end{array}$

$\begin{array}{lllllllllllllllll}5501 & 13 & -3 & 6 & 5 & 1 & 0 & 0 & 4 & 1 & -29.3018 & -68.1353 & -113.270233 & 1 & 1 & 1 & 1\end{array}$

$\begin{array}{llllllllllllllllll}5501 & -3 & 5 & 13 & 6 & 1 & 0 & 0 & 3 & 2 & 44.8696 & -59.0569 & -52.773552 & 0 & 0 & -1 & 0\end{array}$

$$
\begin{aligned}
& \mathrm{v}=-6,3,7,7 \\
& \begin{array}{lllllllllllllllll}
5521 & -7 & -13 & -4 & 0 & 1 & 0 & 0 & 4 & 0 & -65.8889 & -34.3460 & -152.468246 & 0 & -1 & 0 & 0
\end{array} \\
& \begin{array}{llllllllllllllllll}
5521 & -4 & -7 & 0 & -13 & 1 & 0 & 0 & 2 & 2 & 73.9406 & 7.3352 & 5.665450 & -1 & 0 & 0 & 0
\end{array} \\
& \begin{array}{llllllllllllllllll}
5521 & -13 & 0 & -7 & -4 & 1 & 0 & 0 & 3 & 1 & 73.9404 & -7.3351 & -5.665346 & 0 & 0 & 0 & -1
\end{array} \\
& \begin{array}{lllllllllllllllll}
5521 & 0 & -4 & -13 & -7 & 1 & 0 & 0 & 1 & 3 & -65.8890 & 34.3460 & 152.468292 & 0 & 0 & -1 & 0
\end{array}
\end{aligned}
$$

$\mathrm{v}=-8,0,-3,2$

$\begin{array}{lllllllllllllllllll}5531 & 49 & 4 & 29 & 34 & 1 & 0 & 0 & 0 & 1 & 42.3438 & 61.1392 & 55.294338 & 0 & 0 & -1 & 0\end{array}$

$\begin{array}{lllllllllllllllll}5531 & -106 & -16 & -56 & -81 & 1 & 0 & 0 & 0 & 1 & -38.7741 & -63.4631 & -121.423668 & -1 & 0 & 0 & 0\end{array}$

$\begin{array}{lllllllllllllllll}5531 & -81 & -56 & -16 & -106 & 1 & 0 & 0 & 0 & 1 & -38.7741 & 63.4631 & 121.423683 & 0 & 0 & 0 & -1\end{array}$

$\begin{array}{llllllllllllllllllll}5531 & 34 & 29 & 4 & 49 & 1 & 0 & 0 & 0 & 1 & 42.3438 & -61.1392 & -55.294254 & 0 & -1 & 0 & 0\end{array}$

$$
\begin{aligned}
& \mathrm{v}=-8,-2,-2,3 \\
& \begin{array}{lllllllllllllllll}
5581 & 0 & 11 & -3 & 8 & 1 & 0 & 0 & 1 & 0 & -46.7985 & -58.2315 & -128.787552 & 1 & 1 & 1 & 1
\end{array} \\
& \begin{array}{lllllllllllllllll}
5581 & -338 & -165 & -107 & -374 & 1 & 0 & 0 & 3 & 3 & 73.8414 & 11.3336 & 8.725981 & 0 & 0 & -1 & 0
\end{array} \\
& \begin{array}{lllllllllllllllll}
5581 & -374 & -107 & -165 & -338 & 1 & 0 & 0 & 2 & 4 & 73.8414 & -11.3334 & -8.725868 & 0 & -1 & 0 & 0
\end{array} \\
& \begin{array}{llllllllllllllllll}
5581 & 8 & -3 & 11 & 0 & 1 & 0 & 0 & 4 & 2 & -46.7984 & 58.2314 & 128.787537 & 1 & 1 & 1 & 1
\end{array}
\end{aligned}
$$$$
\mathrm{v}=-8,1,-4,2
$$ 


$\begin{array}{llllllllllllllllllllllll}5591 & -251 & 139 & -241 & -16 & & 1 & 0 & 0 & 0 & 3 & 24.4231 & 70.6719 & 70.935616 & 0 & 0 & 0 & -1 \\ 5591 & -1 & 9 & -6 & 4 & 1 & 0 & 0 & 0 & 3 & & 68.9096 & -29.0253 & -22.841253 & 0 & 0 & -1 & 0 & & & \\ 5591 & 4 & -6 & 9 & -1 & 1 & 0 & 0 & 0 & 3 & & 68.9096 & 29.0253 & 22.841263 & 0 & -1 & 0 & 0 & & & & \\ 5591 & -16 & -241 & 139 & -251 & 1 & 0 & 0 & 0 & 3 & 24.4233 & -70.6719 & -70.935547 & -1 & 0 & 0 & 0\end{array}$


$\mathrm{v}=-7,-6,-5,4$

$\begin{array}{lllllllllllllllll}5821 & 75 & 6 & 42 & 53 & 1 & 0 & 0 & 1 & 3 & 30.1957 & 70.0658 & 66.685822 & 0 & 0 & 0 & -1\end{array}$

$\begin{array}{lllllllllllllllll}5821 & 57 & 35 & 13 & 71 & 1 & 0 & 0 & 3 & 1 & -42.1808 & 63.5750 & 123.563522 & -1 & 0 & 0 & 0\end{array}$

$\begin{array}{lllllllllllllllll}5821 & 71 & 13 & 35 & 57 & 1 & 0 & 0 & 2 & 2 & -42.1807 & -63.5750 & -123.563454 & 0 & 0 & 0 & -1\end{array}$

$\begin{array}{llllllllllllllllll}5821 & 53 & 42 & 6 & 75 & 1 & 0 & 0 & 4 & 0 & 30.1958 & -70.0658 & -66.685730 & -1 & 0 & 0 & 0\end{array}$

\begin{tabular}{|c|c|c|c|c|c|c|}
\hline 5851 & $\begin{array}{l}7,-4,5,2 \\
-3-5-12-9\end{array}$ & $\begin{array}{lllll}1 & 0 & 0 & 3 & 2\end{array}$ & 20.6106 & -73.6628 & -74.368561 & $\begin{array}{llll}-1 & 0 & 0 & 0\end{array}$ \\
\hline 351 & $\begin{array}{llll}-12 & -3 & -9 & -5\end{array}$ & $\begin{array}{lllll}1 & 0 & 0 & 4 & 1\end{array}$ & 50.9962 & -57.0120 & -48.187950 & $\begin{array}{lll}-1 & 0 & 0\end{array}$ \\
\hline 351 & $\begin{array}{llll}-5 & -9 & -3 & -12\end{array}$ & $\begin{array}{lllll}1 & 0 & 0 & 1 & 4\end{array}$ & 50.9963 & 57.0122 & 48.187969 & $\begin{array}{llll}0 & 0 & 0 & -1\end{array}$ \\
\hline 351 & $\begin{array}{llll}-9 & -12 & -5 & -3\end{array}$ & 100023 & 20.6106 & 73.6626 & 74.368492 & $\begin{array}{llll}0 & 0 & 0 & -1\end{array}$ \\
\hline
\end{tabular}

$\mathrm{v}=-8,-4,3,0$

$\begin{array}{lllllllllllllllllll}5861 & 71 & -17 & 55 & 27 & 1 & 0 & 0 & 2 & 0 & 76.4881 & 3.2528 & 2.435180 & 1 & 1 & 1 & 1\end{array}$

$\begin{array}{lllllllllllllllllll}5861 & -55 & 6 & -38 & -27 & 1 & 0 & 0 & 1 & 1 & 7.2773 & 76.2105 & 84.545395 & -1 & 0 & 0 & 0\end{array}$

$\begin{array}{lllllllllllllllll}5861 & -27 & -38 & 6 & -55 & 1 & 0 & 0 & 4 & 3 & 7.2774 & -76.2106 & -84.545311 & 0 & 0 & 0 & -1\end{array}$

$\begin{array}{lllllllllllllllll}5861 & 27 & 55 & -17 & 71 & 1 & 0 & 0 & 3 & 4 & 76.4880 & -3.2527 & -2.435099 & 1 & 1 & 1 & 1\end{array}$

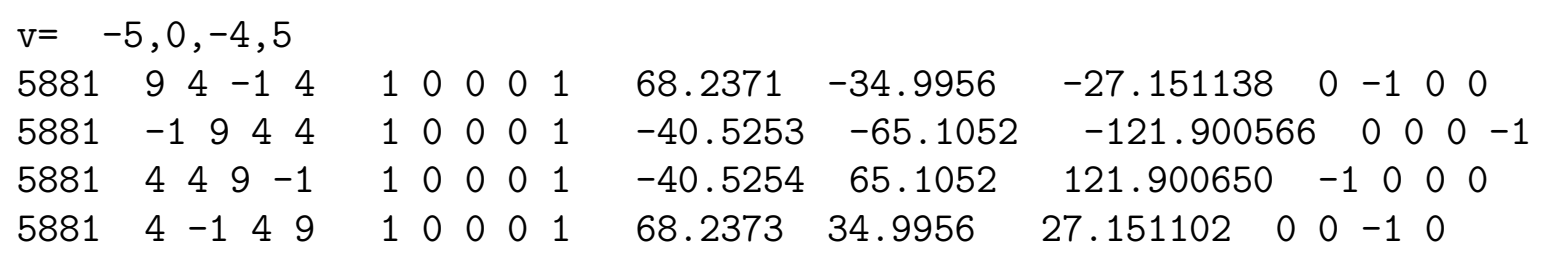

$\mathrm{v}=-5,-1,0,7$
$\begin{array}{lllllllllllllllllll}5981 & 136 & -82 & 135 & 2 & 1 & 0 & 0 & 2 & 4 & 14.9871 & -75.8708 & -78.825943 & 0 & -1 & 0 & 0 \\ 5981 & -25 & -4 & -13 & -17 & 1 & 0 & 0 & 1 & 0 & 21.3702 & -74.3257 & -73.958961 & 1 & 1 & 1 & 1 \\ 5981 & -17 & -13 & -4 & -25 & 1 & 0 & 0 & 4 & 2 & 21.3701 & 74.3257 & 73.959007 & 1 & 1 & 1 & 1 \\ 5981 & 2 & 135 & -82 & 136 & 1 & 0 & 0 & 3 & 3 & 14.9871 & 75.8708 & 78.825974 & 0 & 0 & -1 & 0\end{array}$




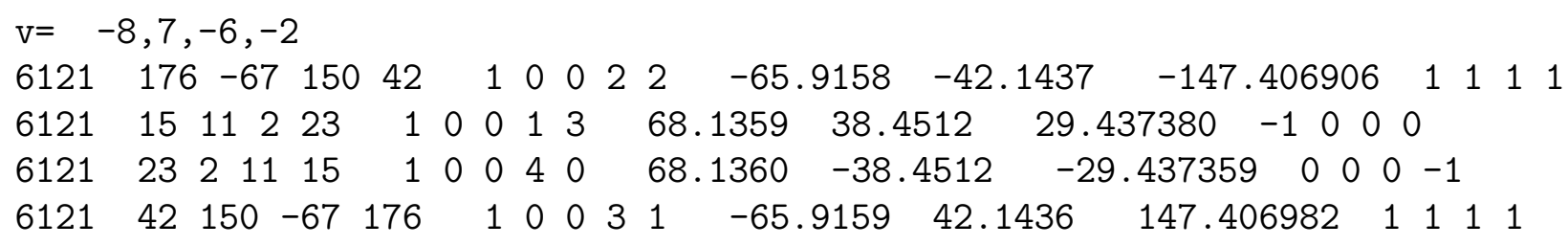


$\begin{array}{llllllllllllllllll}6311 & 230 & -129 & 222 & 13 & 1 & 0 & 0 & 1 & 1 & -23.7686 & 75.8030 & 107.409203 & 0 & -1 & 0 & 0\end{array}$

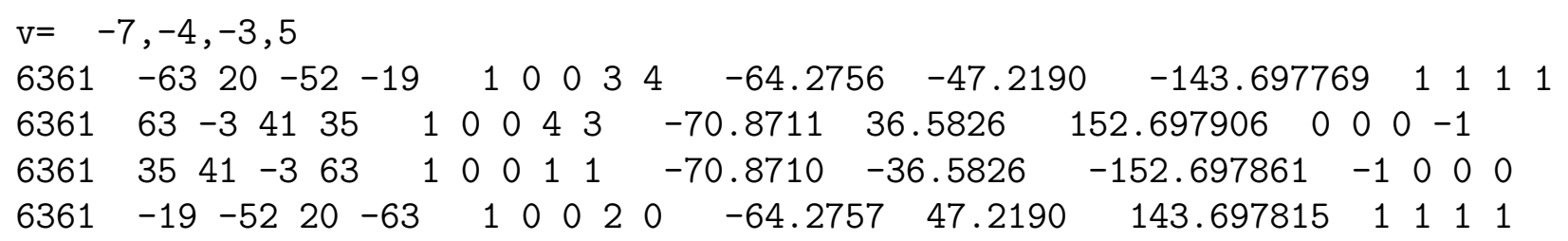




\section{Bibliography}

[Ba] H. Bass: Generators and relations for cyclotomic units. Nagoya Math. J. Vol27(1966) 401-407.

[A. Bear] Alan F. Beardon: The geometry of Discrete groups Graduate Texts in Mathematics, Vol91(1983)ed. Springer-Verlag New York Inc.

[B1] B. C. Berndt, R. J. Evans, K. S. Williams: Gauss and Jacobi SumsCan. Math. Soc. Vol21(1998)ed. John Wiley and sons.

[C-F] J. W. S. Cassels, A. Fröhlich: Algebraic number theory,Proc Inter.Conf. Lond.Math. Soc (1967)351-353.

[J.W.S] J.W.S Cassels:On the determination of generalized Gauss sums,Arch. Math.t.5,(1969)7984.

[J.W.S1] J.W.S Cassels:On Kummer sums,Proc. London Math. Soc. (3)21,(1970)19-27.

[C] A. Cauchy: Methode simple et nouvelle pour la determination complete des sommes alternees formees avec les racines primitives des equations binomes J. de Math. pures et appl., ser 15(1840)154-168

[E] G. Eisenstein:Beweis der allgemeinsten Reziprozitästgesetze zwischen reellen und complexen Zahlen,Monastber. d. preuss. Akad. d. Wiss. zu Berlin (1850)189-190.

[Da] H. Davenport:Multiplicative Number Theory,2nd ed., Springer-Verlag, New York(1980) .

[E.T] T. Estermann: On the sign of Gaussian sum,J.London Math. Soc. 20(1945)66-67.

[I.M. Gel] I. M. Gel'fand, M. I. Graev, I. I. Pyatetskii-Shapiro: The Representation Theory and Automorphic functions Translated from the Russian by K. A. Hirsch,(1969)ed. W.B. Saunders Company .

[G.VN] H. Goldstine and J. Von Neumann: A numerical study of a conjecture of Kummer,Math of comp. $7(1953) 133-134$

[HP] D. R. Heath-Brown and S.J. Patterson : The distribution of Kummer sums at prime arguments,J. reine und angew. Math. 310,(1979)111-130.

[H] D. Hilbert:Theorie des corps de nombres algébriques,edition Jacques Gabay,Sceaux(1991).

[Ha] Habicht:Ein elementarer Beweis des kubischen Reziprozitästgesetzes, Math. Annalen 139(1959-1960)343-345.

[H] D. Hilbert:Theorie des nombres algebriques,Ann.Fac.Sc.Toulouse3. serieNo.3(1911) 
[R-H] R. Hill:Ein geometrischer Beweis eines ReziprozitätsgesetzesDiss Goettingen(1992).

[I.R] K. Ireland, M. Rosen A Classical Introduction to Moderne Number Theory,Graduate Texts in Mathematics,vol. 84 (1982)by Springer Verlag, New York.

[K.L] L. Kronecker: Sur une formule de Gauss,J. de Math. Pures et Appl.(ser. 2)1(1856)392-395.

[KB1] T. Kubota: On a special kind of Dirichlet series,J. Math. Soc. Japan, 20(1968) 193-207

[KB2] T. Kubota: Some results concerning reciprocity laws and real automorphic functions,"1969 Number Theory Institute". p 382-395. -Providence, American Mathematical Society,1971( Proceedings of Symposia in pure Mathematics, 20).

[KuNi] L. Kuipers and Niederreiter: Uniform distribution of sequences. Wiley, New York, (1974).

[K] E.E Kummer: Über die Zerlegung der aus Würzeln der Einheit gebildeten komplexen Zahlen in ihre Primfaktoren,J.R.A.M 35(1847)327-367 or CW vol I(1975)211-251.

[K1] E.E Kummer: Eine Aufgabe, betrefend die Theorie der kubischen Reste,,J.Reine Angew. Math 32(1842)285-286 or CW vol I(1975)143-144.

[K2] E.E Kummer: De residuis cubicis disquitiones nonnullae analyticae,J.Reine Angew. Math 32(1846)341-349 or CW vol I(1975)145-164.

[S.L] S. Lang:Cyclotomic fields, Graduate Texts in Mathematics, vol. 59(1978) by Springer Verlag, New York

[S.L1] S. Lang:Algebraic Number Theory,(1970)by Adison-Wesley Pubblishing Company

[EL] E. Lehmer: On the location of Gauss sums,Math. of Comp.10(1956) 194-202

[F-Lem] F. Lemmermeyer:Reciprocity Laws from Euler to Eisenstein,(2000) Springer Monograph in Mathematics.

[F-L] F. Lemmermeyer: The Euclidean algorithm in algebraic number fields,Expos.Math.13,No.5(1995)385-416.

[H.W.L] H. W. Lenstra:Lectures on Euclidean ring,Bielefeld 1974.

[Lox] J. H. Loxton :Product related to Gauss, ,J. Reine Angew. Math. 268(1974)53-67.

[Lox1] J. H. Loxton :Some conjectures concerning Gauss sums. J. Reine Angew. Math. 297, 153-158 (1978).

[Mat] C. R. Matthews:Gauss sums and elliptic functions, I. The Kummer sum,Invent Math. 52,(1979)163-185; The quartic sum, Invent. Math. 52(1979),23-52.

[Mc.G] A. D. McGettrick :A result in the theory of Weierstrass elliptic functions,Proc. London Math. Soc 3,No.25(1972)41-54.

[Mc.G1] A. D. McGettrickOn the biquadratic Gauss sum. Proc. Camb. Philos. Soc.71, 79-83 (1972).

[G.M] G. Meyerson: period polynomial and Gauss sums for finite fields,Acta Arith.39No. $3(1981) 251-264$. 
[Min] H. Minkowski: Zur Theorie der Einheiten in den algebraischen Zahlkoerpern. Nach. Ges. Wiss. Goettingen, (1900),90-93.

[MON] H. L. Montgomery: Ten lectures on the interface between analytic number theory and harmonic analysis CBMS Regional Conference series, vol. 84, Amer. math. soc., 1994.

[MO] C. Moreno :Sur le problème de Kummer Enseignement Math. 20(1974), 45-51

[Na] Melvyn B. Nathason :Elementary Methods in Number Theory. Graduate Texts in Mathematics. 195,(2000) Springer

[O] J. Ouspensky:Note sur les nombres entiers dependant d'une racine cinquieme de $l$ unite,Mathematische Annalen LXVI1908.

[SJP1] S.J. Patterson : The distribution of general Gauss sums at prime arguments, Advances in analytic number theory, Durham(1979) (ed. H. Halberstam and C. Hooley, Academic Press, London,(1981),Vol. 2, pp 171-182.

[SJP2] S.J. Patterson: The distribution of general Gauss sums and similar arithmetic functions at prime arguments,Proc. London Math. Soc 3,No.54(1987)193-215.

[SJP3] S.J. Patterson : The cubic analogue of the theta series I,J. reine und angew. Math. 296,(1977)125-161.

[SJP4] S.J. Patterson : The cubic analogue of the theta series II,J. reine und angew. Math. 296,(1977)217-220.

[SJP5] S.J. Patterson : On the distribution of Kummer sums,J. reine und angew. Math. 303/304,(1978)126-143.

[SJP6] S.J. Patterson : Gaüss'sche Summe: ein Thema mit variationen Antrittsvorlesung, Goettingen (1981)

[Ram] Ramachandra: On the units of cyclotomic fields, Acta artith. 12,(1966)165-173.

[P] L. S. Pontriagin: Topological Groups,Princeton(1946)

[P.S] P. Samuel:Algebraic theory of numbers,Hermann,Houghton Mifflin,Boston,1970.

[S.I] I. Schur:Über die Gaussschen Summen,Gött. Nachrichten,(1920-210)147-153.

[G-S] C-G.Schmidt:Über die Führer von Gaussschen Summen als Grössencharaktere,J. Number Theorie12,No.3(1980)283-309.

[G-S1] C-G.Schmidt:Grössencharaktere und Relativklassenzahl abelscher Zahlkörper,J. Number Theorie11,No.1(1979)128-159.

[SL] L. Stickelberger:Ub̈er eine Verallgemeinerung der Kreisteilung,Math.Ann.37(1890)321-367.

[F.T1] F. Thaine: Families of irreducible polynomials of Gaussian periods and matrice of cyclotomic number,Math.Comp.69No 232(2000)1653-1666. 
[F.T2] F. Thaine: Gaussian periods that are rational integers ,Preprint, CICMA, Concordia University.

[V] H. Vandivier:Some properties of of a certain system of fundamental units in cyclotomic field Ann.Math. 31 (1930)123-125.

[WR] R. Webster:Convexity,Oxford Science Publication,1994.

[w1] A. Weil: La cyclotomic jadis et naguère,Enseign.Math.20(1974)247-263.

[w2] A. Weil: Jacobi sums as Grössencharaktere,Trans.Amer.Math.Soc. 73(1952)487-495.

[w3] A. Weil: Sommes de Jacobi et caracteres de Hecke,Gött. Nach.1(1974)1-14.

[w4] A.Weil: Basic Number Theory,Springer,1967.

[V] H. Vandivier:Some properties of of a certain system of fundamental units in cyclotomic field Ann.Math. 31 (1930)123-125. 


\section{Lebenslauf von FOSSI TALOM Léopold}

Ich wurde am 05.01.1968 in Bandjoun-yom V, Kamerun, geboren.

Schulausbildung:

1973-1979: Katholische Schule zu Yom mit dem Abschluss Certificat d'Etudes Primaires et Elémentaires (C.E.P.E.).

1979-1984: College d'Enseignement Secondaire Géneral( C.E.S) de Bandjoun mit dem Abschluss Brevet d'Etudes du Premier Cycle ( B. E. P. C.).

1979-1988: Lycée Classique et moderne de Bafoussam mit den Abschlussen, Probatoire Serie C (1987) und Baccalauréat Serie C.(1988)

Studium:

1988-1995: Studium der Mathematik, Informatik und Physik and der Université de Yaoundé, Abschluss: Licence, Maitrise, D.E.A( Diplôme d'Etudes Approfondies)

1994-1996: Teilzeit wissenschalicher Mitarbeiter an der mathematischen Fakultät der universität Yaoundé.

1996-1997: Assistent an der Fakültät für Wirtschaft- und Socialwissenschaft der Université Catholique d'Afrique Centrale.

April-sept 1997: Sprachkurs am Goethe Institut Mannheim mit dem Abschluss P.N.D.S

1997- : Zulassung zur Promotion, Fakültät für Mathematik der Georg August Universität Göttingen. 